\title{
Divergent Synthesis of $\alpha$-Fluorinated Carbonyl and Carboxyl Derivatives by Double Electrophilic Activation of Amides
}

\author{
Amaury Dubart and Gwilherm Evano* \\ Gwilherm.Evano@ulb.be
}

Laboratoire de Chimie Organique, Service de Chimie et PhysicoChimie Organiques, Université Libre de Bruxelles, Avenue F. D. Roosevelt 50, CP160/06, 1050 Brussels, Belgium.

General Information

Experimental Procedures and Characterization Data:

Optimization Tables. .S2

Experimental Procedures and Characterization Data:

Reported Starting Materials

Experimental Procedures and Characterization Data:

Unreported Starting Materials..

Experimental Procedures and Characterization Data:

$\alpha$-Fluoroketones

Experimental Procedures and Characterization Data:

$\alpha$-Fluoroesters.

Experimental Procedures and Characterization Data:

$\alpha$-Fluoroamide.

Experimental Procedures and Characterization Data:

$\alpha$-Fluorocarboxylic Acids.

GC Spectra

Determination of the Absolute Stereochemistry. . .534

${ }^{1} \mathrm{H},{ }^{13} \mathrm{C}$ NMR and ${ }^{19} \mathrm{~F}$ NMR Spectra. . .535 


\section{General Information.}

All reactions were carried out in oven-dried glassware under an argon atmosphere employing standard techniques in handling air-sensitive materials unless otherwise stated.

Trifluoromethanesulfonic anhydride was distilled over $\mathrm{P}_{2} \mathrm{O}_{5}$ prior to use and store under an argon atmosphere at $0{ }^{\circ} \mathrm{C}$. Dichloromethane was freshly distilled from $\mathrm{CaH}_{2}$ under an argon atmosphere. Tetrahydrofuran was freshly distilled from $\mathrm{Na}$ /benzophenone under an argon atmosphere. Ethanol was dried with in-situ activated magnesium turnings and distilled under an argon atmosphere. All other reagents and solvents were used as supplied.

Reactions were magnetically stirred and monitored by thin layer chromatography using Merck-Kiesegel $60 \mathrm{~F}_{254}$ plates. Temperatures of the reaction refer to the temperature of the oil bath. Flash chromatography was performed with silica gel 60 (particle size 35-70 $\mu \mathrm{m}$ ) supplied by Merck. Yields refer to chromatographically and spectroscopically pure compounds unless otherwise stated.

Proton NMR spectra were recorded using an internal deuterium lock at $25^{\circ} \mathrm{C}$, unless otherwise stated, on a Bruker Avance 300 or Jeol JNM-ECZ400R/53 spectrometer. Internal reference of $\delta_{\mathrm{H}} 7.26$ was used for $\mathrm{CDCl}_{3}$, and $\delta_{\mathrm{H}} 3.31$ was used for $\mathrm{CD}_{3} \mathrm{OD}$. Data are presented as follows: chemical shift (in ppm on the $\delta$ scale relative to $\delta_{\text {TMS }}=0$ ), multiplicity ( $s=$ singlet, $d$ $=$ doublet, $t=$ triplet, $q=$ quartet, quint. $=$ quintuplet, sept. $=$ septuplet, $m=$ multiplet, $b r .=$ broad app. $=$ apparent, obs. $=$ observed $)$, coupling constant $(\mathrm{J} / \mathrm{Hz})$ and integration. Carbon-13 NMR spectra were recorded at $100 \mathrm{MHz}$ using $\mathrm{CDCl}_{3}\left(\delta_{\mathrm{C}} 77.16\right)$, or $\mathrm{CD}_{3} \mathrm{OD}\left(\delta_{\mathrm{C}} 49.00\right)$ as internal reference. Fluorine-19 NMR spectra were recorded at $376 \mathrm{MHz}$ using fluorobenzene $\left(\delta_{\mathrm{F}}\right.$ 113.15) as internal reference.

Optical rotations were recorded on an Anton Paar MCP-100 automatic polarimeter at $589 \mathrm{~nm}$ and reported as follows: $[\alpha]_{\mathrm{D}}^{25}$, concentration $(c$ in $\mathrm{g} / 100 \mathrm{~mL}$ ), and solvent. Melting points were recorded on a Stuart Scientific Analogue SMP11. Infrared spectra were recorded on a Bruker Alpha (ATR). High-resolution mass-spectra (HRMS) in positive mode were recorded using a 6520 series quadrupole time-of-flight (Q-TOF) mass spectrometer (Agilent) fitted with a multimode ion source. Gaseous chromatography analyses were recorded on a Thermo Finningan Trace GC apparatus with a ChiraSil-DEX CB $(25 \mathrm{~m}, 0.25 \mathrm{~mm}, 25 \mu \mathrm{m})$ column. 


\section{Experimental Procedures and Characterization Data: Optimization Tables}

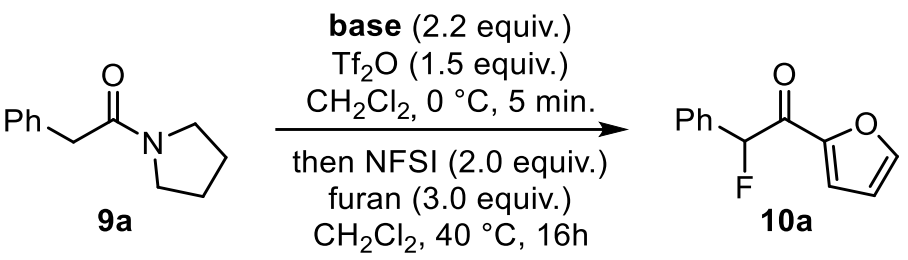

\begin{tabular}{|c|c|c|}
\hline Entry & Base & NMR yield ${ }^{S 1}$ \\
\hline 1 & collidine & $9 \%$ \\
\hline 2 & 2,6-lutidine & $15 \%$ \\
\hline 3 & 2,6-di-tert-butyl-4-methyl-pyridine & $22 \%$ \\
\hline 4 & 2-OMe-pyridine & $16 \%$ \\
\hline 5 & 2-Ph-pyridine & $22 \%$ \\
\hline 6 & 2-F-pyridine & $39 \%$ \\
\hline 7 & 2-Cl-pyridine & $52 \%$ \\
\hline 8 & 2-Br-pyridine & $50 \%$ \\
\hline 9 & 2-I-pyridine & $52 \%$ \\
\hline
\end{tabular}

Table 1: Optimization of the base.

s1 Determined using 1,3,5-trimethoxybenzene as an internal standard. 


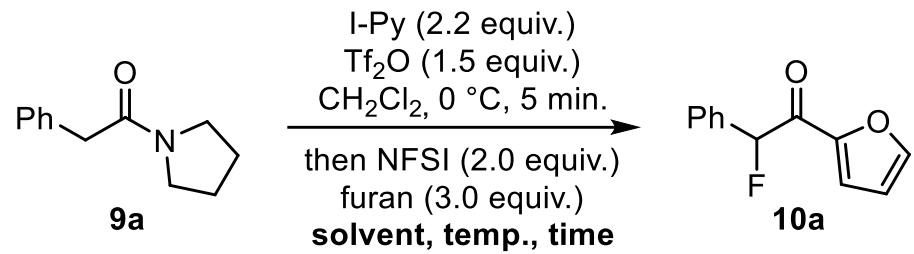

\begin{tabular}{|c|c|c|c|c|}
\hline Entry & Solvent & Time & Temp. & NMR yield $^{\text {s1 }}$ \\
\hline 10 & $\mathrm{CH}_{2} \mathrm{Cl}_{2}$ & $16 \mathrm{~h}$ & $40{ }^{\circ} \mathrm{C}$ & $\mathbf{5 2 \%}$ \\
\hline 11 & $\mathrm{DCE}$ & $16 \mathrm{~h}$ & $40{ }^{\circ} \mathrm{C}$ & $45 \%$ \\
\hline 12 & $\mathrm{CH}_{3} \mathrm{CN}$ & $16 \mathrm{~h}$ & $40{ }^{\circ} \mathrm{C}$ & $38 \%$ \\
\hline 13 & $\mathrm{CHCl}_{3}$ & $16 \mathrm{~h}$ & $40{ }^{\circ} \mathrm{C}$ & $30 \%$ \\
\hline 14 & $\mathrm{CH}_{2} \mathrm{Cl}_{2}$ & $16 \mathrm{~h}$ & $20{ }^{\circ} \mathrm{C}$ & $43 \%$ \\
\hline 15 & $\mathrm{CH}_{2} \mathrm{Cl}_{2}$ & $48 \mathrm{~h}$ & $20{ }^{\circ} \mathrm{C}$ & $52 \%$ \\
\hline 16 & $\mathrm{CH}_{3} \mathrm{CN}$ & $16 \mathrm{~h}$ & $20{ }^{\circ} \mathrm{C}$ & $45 \%$ \\
\hline 17 & $\mathrm{DCE}_{10}$ & $16 \mathrm{~h}$ & $80{ }^{\circ} \mathrm{C}$ & $15 \%$ \\
\hline 19 & $\mathrm{CH}_{3} \mathrm{CN}$ & $16 \mathrm{~h}$ & $60{ }^{\circ} \mathrm{C}$ & $42 \%$ \\
\hline & $\mathrm{CH}_{3} \mathrm{CN}$ & $16 \mathrm{~h}$ & $80{ }^{\circ} \mathrm{C}$ & $46 \%$ \\
\hline
\end{tabular}

Table 2: Optimization of the solvent, temperature, and reaction time.

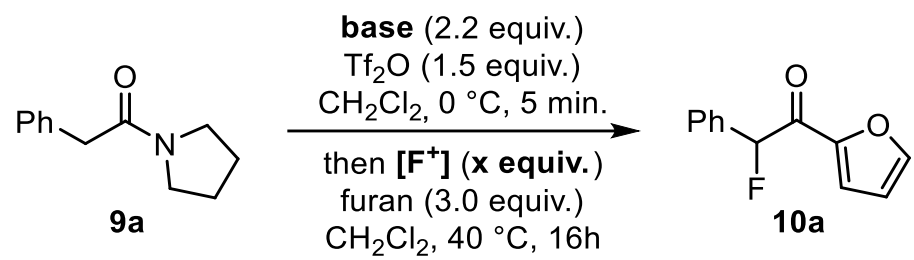

\begin{tabular}{|c|c|c|}
\hline Entry & {$\left[\mathbf{F}^{+}\right]$} & NMR yield $^{\text {s1 }}$ \\
\hline 20 & $\begin{array}{l}\text { SelectFluor }^{\circledR} \\
2.0 \text { equiv. }\end{array}$ & No conv. \\
\hline 21 & $\begin{array}{c}\text { 1-fluoro-sym-collidinium triflate } \\
2.0 \text { equiv. }\end{array}$ & $42 \%$ \\
\hline 22 & $\begin{array}{c}\text { NFSI } \\
1.0 \text { equiv. }\end{array}$ & $7 \%$ \\
\hline 23 & $\begin{array}{c}\text { NFSI } \\
2.0 \text { equiv. }\end{array}$ & $52 \%$ \\
\hline 24 & $\begin{array}{c}\text { NFSI } \\
5.0 \text { equiv. }\end{array}$ & $16 \%$ \\
\hline
\end{tabular}

Table 3: Optimization of the fluorine source and stoichiometry. 


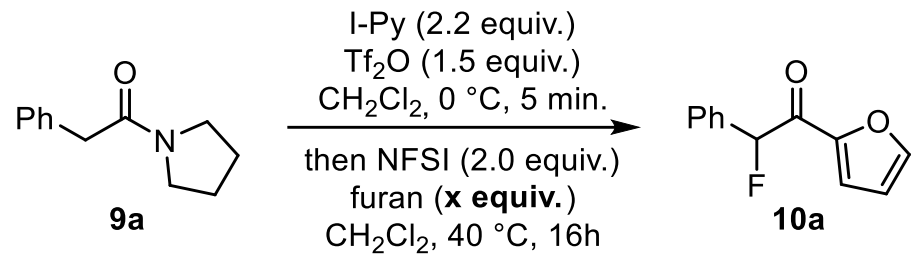

\begin{tabular}{|c|c|c|}
\hline Entry & Equiv. furan & NMR yield $^{\text {s1 }}$ \\
\hline 24 & 1.0 & $42 \%$ \\
\hline 25 & 3.0 & $\mathbf{5 2 \%}$ \\
\hline 26 & 10.0 & $51 \%$ \\
\hline
\end{tabular}

Table 4: Optimization of the stoichiometry of the nucleophile.<smiles>[R]N([R])C(=O)Cc1cccc2ccccc12</smiles>

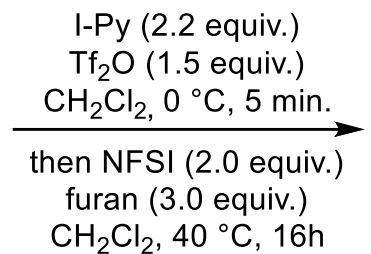<smiles>O=C(c1ccco1)C(F)c1cccc2ccccc12</smiles>

\begin{tabular}{|c|c|c|}
\hline Entry & Amide & NMR yield ${ }^{\text {s1 }}$ \\
\hline 27 & & Traces of product \\
\hline 28 & & $52 \%$ \\
\hline 29 & & $41 \%$ \\
\hline 30 & & $\begin{array}{c}\text { Traces of product } \\
\text { Low conversion (40\%) }\end{array}$ \\
\hline 31 & & $44 \%$ \\
\hline 32 & & I \\
\hline
\end{tabular}

Table 5: Optimization of the starting amide. 


\section{Experimental Procedures and Characterization Data:}

\section{Reported Starting Materials}

Previously reported starting amides $9 a,{ }^{S 2} \mathbf{9 c},{ }^{\mathrm{S2}} \mathbf{9 f},{ }^{\mathrm{S3}} \mathbf{9 g},{ }^{\mathrm{S4}} \mathbf{9 h},{ }^{\mathrm{S3}} \mathbf{9 j},{ }^{\mathrm{S5}} \mathbf{9 l},{ }^{\mathrm{S6}} \mathbf{9 m},{ }^{\mathrm{S2}} \mathbf{9} \mathrm{n}^{\mathrm{S7}}$ and $\mathbf{9} \mathbf{o}^{\mathrm{S8}}$ were prepared according to General procedures I, II or III. Structures were confirmed by comparisons of data reported in the literature.

\footnotetext{
S2 Niu, Z.-J.; Li, L.-H.; Li, X.-S.; Liu, H.-C.; Shi, W.-Y.; Liang, Y.-M. Org. Lett. 2021, 23, 1315.

S3 Jambu, S.; Sivasakthikumaran, R.; Jeganmohan, M. Org. Lett. 2019, 21, 1320

S4 Trillo, P.; Slagbrand, T.; Adolfsson, H. Angew. Chem. Int. Ed. 2018, 57, 12347.

${ }^{55}$ Ning, X.-Q.; Lou, S.-J.; Mao, Y.-J.; Xu, Z.-Y.; Xu, D.-Q. Org. Lett. 2018, 20, 2445

${ }^{56}$ Adler, P.; Teskey, C. J.; Kaiser, D.; Holy, M.; Sitte, H. H.; Maulide, N. Nat. Chem. 2019, 11, 329

S7 Sandoval, B. A.; Clayman, P. D.; Oblinsky, D. G.; Oh, S.; Nakano, Y.; Bird, M.; Scholes, G. D.; Hyster, T. K. J. Am. Chem. Soc. 2020, 143, 1735

s8 de la Torre, A.; Kaiser, D.; Maulide, N. J. Am. Chem. Soc. 2017, 139, 6578
} 


\section{Experimental Procedures and Characterization Data: \\ Unreported Starting Materials}

\section{General procedure I: Synthesis of amides with oxalyl chloride}

To a solution of carboxylic acid $(10.0 \mathrm{mmol})$ in dry dichloromethane $(20 \mathrm{~mL})$ at $0{ }^{\circ} \mathrm{C}$ was added dropwise oxalyl chloride $(950 \mu \mathrm{L}, 11.0 \mathrm{mmol})$ and a catalytic amount of $\mathrm{N}, \mathrm{N}$-dimethylformamide. The resulting solution was warmed to rt, stirred for 1 hour, cooled down to $0{ }^{\circ} \mathrm{C}$ and a solution of the amine $(1.3 \mathrm{~mL}, 15.0 \mathrm{mmol})$ and triethylamine $(2.7 \mathrm{~mL}, 20.0$ $\mathrm{mmol})$ in dry dichloromethane $(6.5 \mathrm{~mL})$ was added dropwise. The resulting mixture was then warmed to rt, stirred overnight, diluted with dichloromethane, washed with water $(20 \mathrm{~mL})$, twice with a $1 \mathrm{M}$ solution of $\mathrm{HCl}(2 \times 20 \mathrm{~mL})$, twice with an aqueous saturated solution of $\mathrm{NaHCO}_{3}(2 \times 20 \mathrm{~mL})$. The organic layer was then dried over $\mathrm{MgSO}_{4}$, filtered and concentrated under reduced pressure. The crude residue was finally purified by flash column chromatography over silica gel to yield the desired amide.

\section{General procedure II: Synthesis of amides with thionyl chloride}

To a solution of carboxylic acid $(17.9 \mathrm{mmol})$ in dry dichloromethane $(70 \mathrm{~mL})$ at $0{ }^{\circ} \mathrm{C}$ was added dropwise thionyl chloride $(1.4 \mathrm{~mL}, 19.3 \mathrm{mmol})$ and a catalytic amount of $\mathrm{N}, \mathrm{N}$-dimethylformamide. The resulting solution was warmed to rt, stirred for 1 hour, cooled down to $0{ }^{\circ} \mathrm{C}$ and a solution of the amine $(3.1 \mathrm{~mL}, 26.4 \mathrm{mmol})$ and triethylamine $(3.4 \mathrm{~mL}, 35.2$ $\mathrm{mmol})$ in dry dichloromethane $(35 \mathrm{~mL})$ was added dropwise. The resulting mixture was then warmed to rt, stirred overnight, diluted with dichloromethane, washed with water $(20 \mathrm{~mL})$, twice with a $1 \mathrm{M}$ solution of $\mathrm{HCl}(2 \times 20 \mathrm{~mL})$, twice with an aqueous saturated solution of $\mathrm{NaHCO}_{3}(2 \times 20 \mathrm{~mL})$. The organic layer was then dried over $\mathrm{MgSO}_{4}$, filtered and concentrated under reduced pressure. The crude residue was finally purified by flash column chromatography over silica gel to yield the desired amide.

\section{General procedure III: Synthesis of amides with thionyl chloride}

To a solution of carboxylic acid $(2.2 \mathrm{mmol})$ in dry dichloromethane $(8.8 \mathrm{~mL})$ at $0{ }^{\circ} \mathrm{C}$ was added dropwise thionyl chloride $(175 \mu \mathrm{L}, 2.4 \mathrm{mmol})$ and a catalytic amount of $\mathrm{N}, \mathrm{N}$-dimethylformamide. The resulting solution was warmed to rt, stirred for 1 hour, cooled 
down to $0{ }^{\circ} \mathrm{C}$ and a solution of the amine $(2.0 \mathrm{mmol})$ and triethylamine $(832 \mu \mathrm{L}, 6.0 \mathrm{mmol})$ in dry dichloromethane $(4.0 \mathrm{~mL})$ was added dropwise. The resulting mixture was then warmed to rt, stirred overnight, diluted with dichloromethane, washed with water $(20 \mathrm{~mL})$, twice with a $1 \mathrm{M}$ solution of $\mathrm{HCl}(2 \times 20 \mathrm{~mL})$, twice with an aqueous saturated solution of $\mathrm{NaHCO}_{3}(2 \times 20$ $\mathrm{mL}$ ).The organic layer was then dried over $\mathrm{MgSO}_{4}$, filtered and concentrated under reduced pressure. The crude residue was finally purified by flash column chromatography over silica gel to yield the desired amide.

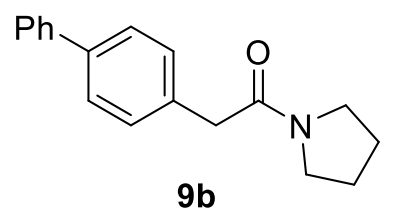

2-([1,1'-Biphenyl]-4-yl)-1-(pyrrolidin-1-yl)ethan-1-one 9b. Prepared according to general procedure I. Yield: 74\% (3.9 g, $14.7 \mathrm{mmol})$. Solvent system for flash column chromatography: petroleum ether/EtOAc: 20/80; Yellowish solid; $\mathrm{Mp}: 83^{\circ} \mathrm{C} ;{ }^{1} \mathrm{H} \mathrm{NMR}\left(400 \mathrm{MHz}, \mathrm{CDCl}_{3}\right): \delta 7.60-$ $7.53(\mathrm{~m}, 4 \mathrm{H}), 7.45-7.40(\mathrm{~m}, 2 \mathrm{H}), 7.38-7.34(\mathrm{~m}, 3 \mathrm{H}), 3.69(\mathrm{~s}, 2 \mathrm{H}), 3.51(\mathrm{t}, J=6.9 \mathrm{~Hz}, 2 \mathrm{H}), 3.46(\mathrm{t}$, $J=6.7 \mathrm{~Hz}, 2 \mathrm{H}$ ), 1.94 (app. p, $J=6.7 \mathrm{~Hz}, 2 \mathrm{H}$ ), 1.85 (app. p, $J=6.7 \mathrm{~Hz}, 2 \mathrm{H}) ;{ }^{13} \mathrm{C} \mathrm{NMR}(100 \mathrm{MHz}$, $\left.\mathrm{CDCl}_{3}\right): \delta 169.5,140.9,139.7,134.1,129.5,128.8,127.4,127.3,127.1,47.0,46.1,42.0,26.3$, 24.5; IR (ATR): $v_{\max } 2971,2874,1636,1487,1429,1340,1190,864,821,758,697 \mathrm{~cm}^{-1}$; ESI-HRMS m/z calcd for $\mathrm{C}_{18} \mathrm{H}_{20} \mathrm{NO}[\mathrm{M}+\mathrm{H}]^{+}$266.1539, found 266.1545 .

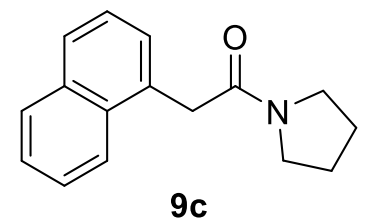

2-(Naphthalen-1-yl)-1-(pyrrolidin-1-yl)ethan-1-one 9c. Prepared according to general procedure I. Yield: 73\% (2.8 g, $11.7 \mathrm{mmol})$. Solvent system for flash column chromatography: petroleum ether/EtOAc: 20/80; Off-white solid; $\mathrm{Mp}: 42{ }^{\circ} \mathrm{C} ;{ }^{1} \mathrm{H} \mathrm{NMR}\left(400 \mathrm{MHz}, \mathrm{CDCl}_{3}\right): \delta 8.06$ (d, $J=8.1 \mathrm{~Hz}, 1 \mathrm{H}$ ), 7.85 (d, $J=7.5 \mathrm{~Hz}, 1 \mathrm{H}), 7.76$ (d, $J=8.1 \mathrm{~Hz}, 1 \mathrm{H}), 7.56-7.46(\mathrm{~m}, 2 \mathrm{H}), 7.44-7.39$ (m, 1H), 7.37-7.34 (m, 1H), $4.08(\mathrm{~s}, 2 \mathrm{H}), 3.54(\mathrm{t}, J=6.7 \mathrm{~Hz}, 2 \mathrm{H}), 3.40(\mathrm{t}, J=6.1 \mathrm{~Hz}, 2 \mathrm{H}), 1.92-$ $1.77(\mathrm{~m}, 4 \mathrm{H}) ;{ }^{13} \mathrm{C}$ NMR $\left(100 \mathrm{MHz}, \mathrm{CDCl}_{3}\right): \delta 169.6,134.0,132.4,131.4,128.8,127.7,126.8$, 
126.4, 125.8, 125.6, 124.0, 47.1, 46.2, 40.1, 26.3, 24.4; IR (ATR): $v_{\max } 3059,2970,2873,1638$, 1425, 1191, 1168, $788 \mathrm{~cm}^{-1}$; ESI-HRMS m/z calcd for $\mathrm{C}_{16} \mathrm{H}_{18} \mathrm{NO}[\mathrm{M}+\mathrm{H}]^{+}$240.1383, found 240.1379 .

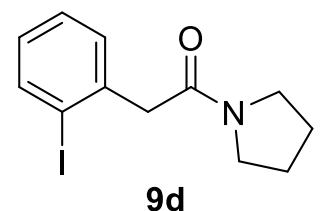

2-(2-lodophenyl)-1-(pyrrolidin-1-yl)ethan-1-one 9d. Prepared according to general procedure II. Yield: 83\% (5.0 g, $15.9 \mathrm{mmol})$. Solvent system for flash column chromatography: petroleum ether/EtOAc: 30/70; Yellow solid; Mp: $39{ }^{\circ} \mathrm{C}$; ${ }^{1} \mathrm{H} \mathrm{NMR}\left(400 \mathrm{MHz}, \mathrm{CDCl}_{3}\right): \delta 7.82$ (d, J $=7.8 \mathrm{~Hz}, 1 \mathrm{H}), 7.31-7.28(\mathrm{~m}, 2 \mathrm{H}), 6.97-6.89(\mathrm{~m}, 1 \mathrm{H}), 3.74(\mathrm{~s}, 2 \mathrm{H}), 3.51(\mathrm{t}, J=6.9 \mathrm{~Hz}, 2 \mathrm{H}), 3.47(\mathrm{t}$, $J=6.7 \mathrm{~Hz}, 2 \mathrm{H}$ ), 1.95 (app. p, $J=6.5 \mathrm{~Hz}, 2 \mathrm{H}$ ), 1.87 (app. p, $J=6.5 \mathrm{~Hz}, 2 \mathrm{H}) ;{ }^{13} \mathrm{C} \mathrm{NMR}(100 \mathrm{MHz}$, $\left.\mathrm{CDCl}_{3}\right): \delta 168.6,139.4,138.8,130.2,128.6,128.6,101.3,47.0$ (2C), 46.1, 26.3, 24.5; IR (ATR): $V_{\max } 2969,2871,1639,1433,1340,1190,1168,1013,748,648 \mathrm{~cm}^{-1}$; ESI-HRMS m/z calcd for $\mathrm{C}_{12} \mathrm{H}_{15} \mathrm{INO}[\mathrm{M}+\mathrm{H}]^{+}$316.0193, found 316.0188.

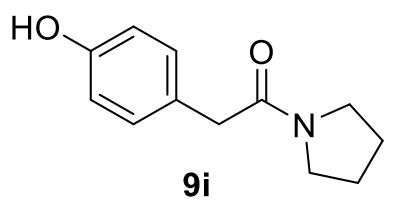

2-(4-Hydroxyphenyl)-1-(pyrrolidin-1-yl)ethan-1-one 9i. Prepared according to general procedure II. Yield: $55 \%$ (1.14 g, $5.5 \mathrm{mmol})$. Solvent system for flash column chromatography: petroleum ether/EtOAc: 20/80; White solid; Mp: $56{ }^{\circ} \mathrm{C}$; ${ }^{1} \mathrm{H}$ NMR (400 MHz, DMSO-d6): $\delta 9.20$ (s, 1H), $7.01(\mathrm{~d}, J=8.5 \mathrm{~Hz}, 2 \mathrm{H}), 6.67(\mathrm{~d}, J=8.4 \mathrm{~Hz}, 2 \mathrm{H}), 3.46(\mathrm{~s}, 2 \mathrm{H}), 3.42(\mathrm{t}, J=6.8 \mathrm{~Hz}, 2 \mathrm{H}), 3.27$ (t, $J=6.8 \mathrm{~Hz}, 2 \mathrm{H}), 1.88-1.80(\mathrm{~m}, 2 \mathrm{H}), 1.78-1.70(\mathrm{~m}, 2 \mathrm{H}) ;{ }^{13} \mathrm{C}$ NMR (100 MHz, DMSO-d6): $\delta 169.9$, 156.7, 131.0, 126.6, 115.9, 47.1, 46.3, 41.1, 40.9, 40.7, 40.4, 40.2, 40.0, 39.8, 26.6, 24.8; IR (ATR): $v_{\max } 3871,3306,3153,1624,1612,1591,1446,1269,1235,800,715,685 \mathrm{~cm}^{-1}$. 


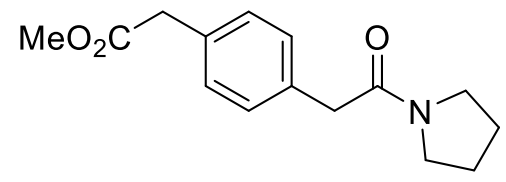

$9 k$

Methyl 2-(4-oxoethylphenyl)-1-(pyrrolidin-1-yl)ethan-1-one 9k. Prepared according to general procedure I. Yield: $73 \%(660 \mathrm{mg}, 2.52 \mathrm{mmol})$. Solvent system for flash column chromatography: petroleum ether/EtOAc: 50/50; Pale yellow oil; ${ }^{1} \mathrm{H} \mathrm{NMR}\left(400 \mathrm{MHz}, \mathrm{CDCl}_{3}\right): \delta$ 7.25-7.20 (m, 4H), $3.68(\mathrm{~s}, 3 \mathrm{H}), 3.62(\mathrm{~s}, 2 \mathrm{H}), 3.59(\mathrm{~s}, 2 \mathrm{H}), 3.47(\mathrm{t}, J=6.8 \mathrm{~Hz}, 2 \mathrm{H}), 3.42(\mathrm{t}, J=6.7$ $\mathrm{Hz}, 2 \mathrm{H}), 1.95-1.78(\mathrm{~m}, 4 \mathrm{H}) ;{ }^{13} \mathrm{C} \mathrm{NMR}\left(100 \mathrm{MHz} \mathrm{CDCl}_{3}\right): \delta 172.2,169.5,133.9,132.5,129.6$, 129.3, 77.5, 77.2, 76.8, 52.1, 47.0, 46.0, 42.0, 40.9, 26.3, 24.5; IR (ATR): $v_{\max } 2980,2950,2877$, $1732,1434,1261,1142,1010,813,625 \mathrm{~cm}^{-1}$.

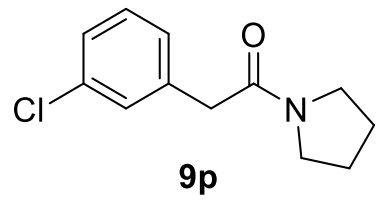

2-(3-Chlorophenyl)-1-(pyrrolidin-1-yl)ethan-1-one 9p. Prepared according to general procedure II. Yield: 53 \% (2.1 g, $9.4 \mathrm{mmol})$. Solvent system for flash column chromatography: petroleum ether/EtOAc: 30/70; White solid; $\mathrm{Mp}: 47^{\circ} \mathrm{C} ;{ }^{1} \mathrm{H} \mathrm{NMR}\left(400 \mathrm{MHz}, \mathrm{CDCl}_{3}\right): \delta 7.27-7.13$ (m, 4H), $3.59(\mathrm{~s}, 2 \mathrm{H}), 3.46(\mathrm{t}, J=6.9 \mathrm{~Hz}, 2 \mathrm{H}), 3.40(\mathrm{t}, J=6.8 \mathrm{~Hz}, 2 \mathrm{H}), 1.92$ (app. p, J=6.4 Hz, 2H), 1.82 (app. p, $J=6.5 \mathrm{~Hz}, 2 \mathrm{H}$ ); ${ }^{13} \mathrm{C}$ NMR $\left(100 \mathrm{MHz}, \mathrm{CDCl}_{3}\right): \delta 168.8,137.0,134.4,129.8,129.3$, 127.4, 127.0, 47.0, 46.1, 41.8, 26.2, 24.4; IR (ATR): $v_{\max } 2971,2875,1636,1475,1191,865$, 770, $685 \mathrm{~cm}^{-1}$; ESI-HRMS m/z calcd for $\mathrm{C}_{12} \mathrm{H}_{15}{ }^{35} \mathrm{CINO}[\mathrm{M}+\mathrm{H}]^{+}$224.0837, found 224.0832.<smiles>C[C@H](c1ccccc1)N(C(=O)Cc1cccc2ccccc12)c1ccccc1</smiles>

2-(Naphthalen-1-yl)-1-((S)-bis((S)-1-phenylethyl)-1-amine)ethan-1-one $\quad 9 a *$ Prepared according to general procedure II. Yield: 22\% (156 mg, $396 \mu \mathrm{mol})$. Solvent system for flash column chromatography: petroleum ether/EtOAc: 20/80; Yellow oil; $[\alpha]_{\mathrm{D}}^{25}-150$ (c 1.0, $\mathrm{CHCl}_{3}$ ); 
${ }^{1} \mathrm{H}$ NMR (400 MHz, tetrachloroethane-d2, $\left.80{ }^{\circ} \mathrm{C}\right): \delta 7.80(\mathrm{dd}, J=7.5$ and $1.8 \mathrm{~Hz}, 1 \mathrm{H}), 7.71(\mathrm{~d}, J$ $=8.2 \mathrm{~Hz}, 1 \mathrm{H}), 7.62(\mathrm{~d}, J=8.2 \mathrm{~Hz}, 1 \mathrm{H}), 7.45-7.31(\mathrm{~m}, 3 \mathrm{H}), 7.21-7.13(\mathrm{~m}, 7 \mathrm{H}), 7.09-6.98(\mathrm{~m}, 4 \mathrm{H})$, 5.17 (d, $J=7.2 \mathrm{~Hz}, 2 \mathrm{H}$ ), 3.97 (d, $J=16.0 \mathrm{~Hz}, 1 \mathrm{H}$ ), 3.90 (d, $J=16.0 \mathrm{~Hz}, 1 \mathrm{H}$ ), 1.70 (d, J= $7.1 \mathrm{~Hz}$, $6 \mathrm{H}) ;{ }^{13} \mathrm{C}$ NMR (100 MHz, tetrachloroethane-d2, $\left.80{ }^{\circ} \mathrm{C}\right): \delta 171.0,141.4,134.1,132.6,132.5$, $128.9,128.5,127.9,127.8,127.5,127.5,126.3,125.9,125.6,124.1,53.8,40.6,18.8$; IR (ATR): $V_{\max } 3056,3031,2977,2936,1639,1495,1428,1377,1316,1205,1162,1093,1023,910,785$, 730, 697, $646 \mathrm{~cm}^{-1}$; ESI-HRMS m/z calcd for $\mathrm{C}_{28} \mathrm{H}_{28} \mathrm{NO}[\mathrm{M}+\mathrm{H}]^{+} 394.2165$, found 394.2170.

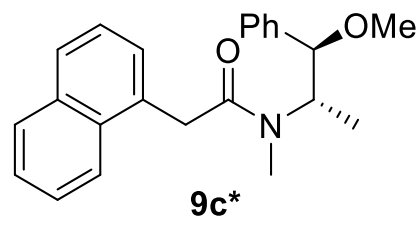

\section{2-(Naphthalen-1-yl)-1-((1R,2S)-1-methoxy- $N, N$-methyl-1-phenylpropan-2-amine)ethan-1-}

one $9 c^{*}$. To a solution of sodium hydride $(60 \% \mathrm{w} / \mathrm{w}, 250 \mathrm{mg}, 6.3 \mathrm{mmol})$ in dry THF $(85 \mathrm{~mL})$ at $0{ }^{\circ} \mathrm{C}$ was added dropwise a solution of 2-(Naphthalen-1-yl)-1-((1R,2S)-2-(methylamino)-1phenylpropan-1-ol)ethan-1-one $(2.1 \mathrm{~g}, 6.3 \mathrm{mmol})$ in dry THF $(17 \mathrm{~mL})$. The solution was stirred for 10 minutes at $0{ }^{\circ} \mathrm{C}$ and methyl iodide $(580 \mu \mathrm{L}, 9.4 \mathrm{mmol})$ was added dropwise. The resulting mixture was then warmed to rt, stirred overnight and stopped by addition of an aqueous saturated solution of $\mathrm{NH}_{4} \mathrm{Cl}$. The aqueous layer was extracted twice with $\mathrm{Et}_{2} \mathrm{O}(2 \times 50 \mathrm{~mL})$ and the organic layer was then dried over $\mathrm{MgSO}_{4}$, filtered and concentrated under reduced pressure. The crude residue was finally purified by flash column chromatography over silica gel (petroleum ether/EtOAc: 80/20) yielding the desired amide as a yellow sticky oil (1.21 g, $3.5 \mathrm{mmol}, 56 \%)$. Obtained as a mixture of rotamers in a ratio of $65 / 35 .[\alpha]_{\mathrm{D}}^{25}-30\left(c 1.3, \mathrm{CHCl}_{3}\right)$; ${ }^{1} \mathrm{H}$ NMR $\left(400 \mathrm{MHz}, \mathrm{CDCl}_{3}\right): \delta$ 7.88-7.82 (m, 1.06H), 7.78-7.73 (m, 1.94H), 7.53-7.44 (m, 1.94H), 7.41-7.31 (m, 4.85H), 7.26-7.23 (m, 0.63H), $7.16(\mathrm{~d}, J=7.1 \mathrm{~Hz}, 0.34 \mathrm{H}$, minor rotamer), 6.99 (d, $J=7.0 \mathrm{~Hz}, 0.63 \mathrm{H}$, major rotamer), 4.88-4.79 $(\mathrm{m}, 0.63 \mathrm{H}$, major rotamer), $4.35(\mathrm{~d}, J=6.4 \mathrm{~Hz}$, $0.63 \mathrm{H}$, major rotamer), $4.11(\mathrm{~d}, J=7.6 \mathrm{~Hz}, 0.34 \mathrm{H}$, minor rotamer), 4.09-4.05 (obs. $\mathrm{m}, 0.34 \mathrm{H}$, minor rotamer), 4.03 (obs. $d, J=16.1 \mathrm{~Hz}, 0.63 \mathrm{H}$, major rotamer), 3.97 (d, $J=16.1 \mathrm{~Hz}, 0.63 \mathrm{H}$, major rotamer), $3.80(\mathrm{~d}, J=16.4 \mathrm{~Hz}, 0.34 \mathrm{H}$, minor rotamer), $3.70(\mathrm{~d}, J=16.2 \mathrm{~Hz}, 0.34 \mathrm{H}$, minor rotamer), $3.25(\mathrm{~s}, 1.02 \mathrm{H}$, minor rotamer), 3.24 (s, 1.89H, major rotamer), 2.93 (s, $1.02 \mathrm{H}$, minor rotamer), 2.90 (s, 1.89H, major rotamer), 1.27 (d, $\left.2.91 \mathrm{H}) ;{ }^{13} \mathrm{C} \mathrm{NMR} \mathrm{(100} \mathrm{MHz,} \mathrm{CDCl}_{3}\right): \delta 171.0$ 
(minor rotamer), 171.0 (major rotamer), 139.4 (2C), 133.8 (major rotamer), 133.8 (minor rotamer), 132.1 (minor rotamer), 132.1 (major rotamer), 131.8 (minor rotamer), 131.4 (major rotamer), 128.8 (minor rotamer), 128.8 (2C), 128.6 (minor rotamer), 128.4 (minor rotamer), 128.3 (major rotamer), 127.8 (major rotamer), 127.6 (minor rotamer), 127.5 (major rotamer), 127.3 (major rotamer), 127.0 (major rotamer), 126.6 (minor rotamer), 126.2 (major rotamer), 126.2 (minor rotamer), 125.7 (major rotamer), 125.7 (major rotamer), 125.6 (minor rotamer), 125.4 (minor rotamer), 123.7 (minor rotamer), 123.5 (major rotamer), 86.3 (major rotamer), 85.4 (minor rotamer), 58.3 (minor rotamer), 57.2 (major rotamer), 57.1 (major rotamer), 54.6 (minor rotamer), 39.0 (major rotamer), 38.2 (minor rotamer), 28.4 (major rotamer), 27.0 (minor rotamer), 15.0 (minor rotamer), 12.8 (major rotamer); IR (ATR): $v_{\max } 2980,2935,2821$, $1642,1451,1398,1094,787,761,702 \mathrm{~cm}^{-1}$; ESI-HRMS m/z calcd for $\mathrm{C}_{24} \mathrm{H}_{26} \mathrm{NO}_{4}\left[\mathrm{M}+\mathrm{HCOO}^{-}\right.$ 392.1867, found 392.1846.

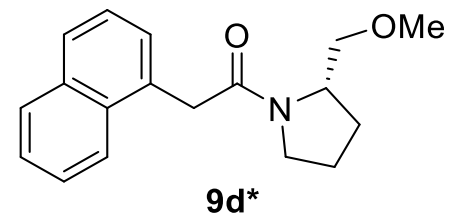

2-(Naphthalen-1-yl)-1-((S-2-methoxymethyl)pyrrolidin-1-yl)ethan-1-one 9d*. Prepared according to general procedure III. Yield: 93\% (603 mg, $2.13 \mathrm{mmol})$. Solvent system for flash column chromatography: petroleum ether/EtOAc: 20/80; Yellow solid; Mp: $62{ }^{\circ} \mathrm{C} ;[\alpha]_{\mathrm{D}}^{25}-40$ (c 1.9, $\left.\mathrm{CHCl}_{3}\right) ;{ }^{1} \mathrm{H}$ NMR $\left(400 \mathrm{MHz}\right.$, DMSO- $\left.d_{6}, 80^{\circ} \mathrm{C}\right): \delta 8.04-8.01(\mathrm{~m}, 1 \mathrm{H}), 7.97-7.87(\mathrm{~m}, 1 \mathrm{H}), 7.82$ (d, $J=8.2 \mathrm{~Hz}, 1 \mathrm{H}), 7.59-7.48(\mathrm{~m}, 2 \mathrm{H}), 7.45(\mathrm{dd}, J=8.2$ and $7.0 \mathrm{~Hz}, 1 \mathrm{H}), 7.38(\mathrm{~d}, J=6.1 \mathrm{~Hz}, 1 \mathrm{H})$, 4.31-4.00 (m, 3H), 3.62-3.42 (m, 3H), $3.36(\mathrm{dd}, J=9.5$ and $6.8 \mathrm{~Hz}, 1 \mathrm{H}), 3.27$ (br. s, 3H), 1.99$1.79(\mathrm{~m}, 4 \mathrm{H}) ;{ }^{13} \mathrm{C}$ NMR $\left(100 \mathrm{MHz}\right.$, DMSO- $\left.d_{6}, 80{ }^{\circ} \mathrm{C}\right): \delta 168.5,133.1(2 \mathrm{C}), 131.9,127.9,126.7$, $126.5,125.4,125.0,124.9,123.8,71.7,58.0,55.9,46.6,38.7,26.9,23.3$; IR (ATR): $v_{\max } 2877$, $1639,1413,1195,1111,968,791,733 \mathrm{~cm}^{-1}$; ESI-HRMS m/z calcd for $\mathrm{C}_{18} \mathrm{H}_{22} \mathrm{NO}_{2}[\mathrm{M}+\mathrm{H}]^{+}$ 284.1645, found 284.1640 . 


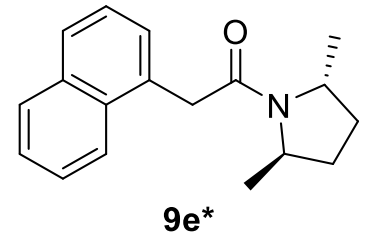

2-(Naphthalen-1-yl)-1-((2R,5R)-2,5-dimethylpyrrolidin-1-yl)ethan-1-one 9e*. Prepared according to general procedure III. Yield: 52\% (294 mg, $1.10 \mathrm{mmol})$. Solvent system for flash column chromatography: petroleum ether/EtOAc: 30/70; Colorless oil; $[\alpha]_{\mathrm{D}}^{25}-17$ (c 1.2, $\left.\mathrm{CHCl}_{3}\right) ;{ }^{1} \mathrm{H}$ NMR $\left(400 \mathrm{MHz}, \mathrm{CDCl}_{3}\right): \delta$ 7.94-7.90 (m, $\left.1 \mathrm{H}\right)$, 7.87-7.84 (m, 1H), 7.78-7.75 (m, $\left.1 \mathrm{H}\right)$, 7.54-7.45 (m, 2H), 7.44-7.37 (m, 2H), $4.34(\mathrm{dt}, J=13.3$ and $6.5 \mathrm{~Hz}, 1 \mathrm{H}), 4.19(\mathrm{~d}, J=15.7 \mathrm{~Hz}, 1 \mathrm{H})$, $4.09(\mathrm{dt}, J=13.0$ and $6.4 \mathrm{~Hz}, 1 \mathrm{H}), 4.02(\mathrm{~d}, J=15.7 \mathrm{~Hz}, 1 \mathrm{H}), 2.25-2.10(\mathrm{~m}, 2 \mathrm{H}), 1.65-1.48(\mathrm{~m}, 2 \mathrm{H})$, $1.27(\mathrm{~d}, J=6.4 \mathrm{~Hz}, 3 \mathrm{H}), 1.22(\mathrm{~d}, J=6.3 \mathrm{~Hz}, 3 \mathrm{H}) ;{ }^{13} \mathrm{C} \mathrm{NMR}\left(100 \mathrm{MHz}, \mathrm{CDCl}_{3}\right): \delta$ 169.6, 134.0, $132.4,132.3,128.9,127.6,126.6,126.3,125.8,125.7,123.7,54.0,53.6,39.5,31.1,29.2$, 22.0, 19.1; IR (ATR): $v_{\max }$ 2967, 2360, 1637, 1401, 1205, 783, $732 \mathrm{~cm}^{-1}$; ESI-HRMS m/z calcd for $\mathrm{C}_{18} \mathrm{H}_{22} \mathrm{NO}[\mathrm{M}+\mathrm{H}]^{+} 268.1696$, found 268.1712 .

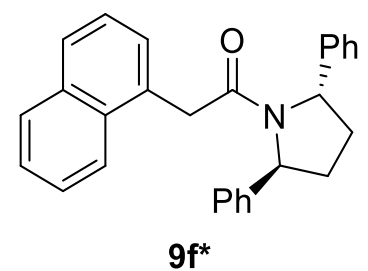

2-(Naphthalen-1-yl)-1-((2R,5R)-2,5-diphenylpyrrolidin-1-yl)ethan-1-one 9f*. Prepared according to general procedure III. Yield: $58 \%$ (743 mg, $1.90 \mathrm{mmol})$. Solvent system for flash column chromatography: petroleum ether/EtOAc: 80/20 to 70/30; Yellowish solid; Mp: 175 ${ }^{\circ} \mathrm{C} ;[\alpha]_{\mathrm{D}}^{25}-68\left(c \quad 0.7, \mathrm{CHCl}_{3}\right) ;{ }^{1} \mathrm{H}$ NMR $\left(400 \mathrm{MHz}^{\mathrm{CDCl}} 3\right): \delta 7.84(\mathrm{dd}, J=8.1$ and $1.4 \mathrm{~Hz}, 1 \mathrm{H}), 7.76$ $(\mathrm{d}, J=8.1 \mathrm{~Hz}, 1 \mathrm{H}), 7.72(\mathrm{~d}, J=8.6 \mathrm{~Hz}), 7.50-7.29(\mathrm{~m}, 9 \mathrm{H}), 7.22-7.17(\mathrm{~m}, 4 \mathrm{H}), 7.08-7.05(\mathrm{~m}, 2 \mathrm{H})$, $5.58(\mathrm{~d}, J=7.6 \mathrm{~Hz}, 1 \mathrm{H}), 5.22(\mathrm{~d}, J=7.2 \mathrm{~Hz}, 1 \mathrm{H}), 3.93(\mathrm{~d}, J=15.6 \mathrm{~Hz}, 1 \mathrm{H}), 3.78(\mathrm{~d}, J=15.6 \mathrm{~Hz}$, $1 \mathrm{H}), 2.51-2.36(\mathrm{~m}, 2 \mathrm{H}), 1.80-1.72(\mathrm{~m}, 1 \mathrm{H}), 1.72-1.64(\mathrm{~m}, 1 \mathrm{H}) ;{ }^{13} \mathrm{C} \mathrm{NMR}\left(100 \mathrm{MHz}, \mathrm{CDCl}_{3}\right): \delta$ 170.6, 143.7, 143.4, 134.0, 132.4, 131.4, 129.2, 128.7, 128.4, 127.8, 127.7, 127.7, 126.8, 126.2, 125.8 (2C), 125.5, 125.4, 124.2, 62.5, 62.4, 40.3, 33.2, 30.2; IR (ATR): $v_{\max } 3058,2974,1651$, $1393,1170,783,752,733,701 \mathrm{~cm}^{-1}$; ESI-HRMS m/z calcd for $\mathrm{C}_{28} \mathrm{H}_{26} \mathrm{NO}[\mathrm{M}+\mathrm{H}]^{+} 392.2004$, found 392.2000 . 


\section{Experimental Procedures and Characterization Data: \\ $\alpha$-Fluoroketones}

\section{General procedure:}

To a solution of amide $(1.0 \mathrm{mmol})$ and 2-iodopyridine $(230 \mu \mathrm{L}, 2.2 \mathrm{mmol})$ in dry dichloromethane $(3 \mathrm{~mL})$ at $0{ }^{\circ} \mathrm{C}$ was added dropwise triflic anhydride $(250 \mu \mathrm{L}, 1.5 \mathrm{mmol})$. The resulting brown solution was stirred for 5 minutes at $0{ }^{\circ} \mathrm{C}$ then $\mathrm{N}$-fluorobenzenesulfonimide $(631 \mathrm{mg}, 2.0 \mathrm{mmol})$ and a solution of the nucleophile $(3.0 \mathrm{mmol})$ in dry dichloromethane were successively added in one portion. The dark brown reaction mixture was stirred at $40{ }^{\circ} \mathrm{C}$ overnight, cooled down to rt and stopped by addition of a saturated solution of $\mathrm{NaHCO}_{3}(5$ $\mathrm{mL}$ ). The biphasic mixture was vigorously stirred for 1 hour then diluted with dichloromethane. The organic phase was washed with an aqueous saturated solution of $\mathrm{NaHCO}_{3}(10 \mathrm{~mL})$, with a $1 \mathrm{M}$ solution of $\mathrm{HCl}(10 \mathrm{~mL})$, dried over $\mathrm{MgSO}_{4}$, filtered and concentrated under reduced pressure. The crude residue was finally purified by flash column chromatography over silica gel to yield the desired fluorinated ketone.

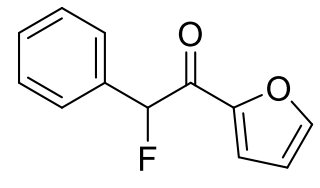

$10 \mathrm{a}$

2-Fluoro-1-(furan-2-yl)-2-phenylethan-1-one 10a. Yield: 52\% (106 mg, $519 \mu \mathrm{mol})$. Solvent system for flash column chromatography: petroleum ether/EtOAc: 95/5; White solid; Mp: 54 ${ }^{\circ} \mathrm{C} ;{ }^{1} \mathrm{H}$ NMR $\left(400 \mathrm{MHz}, \mathrm{CDCl}_{3}\right): \delta$ 7.64-7.62 (m, $\left.1 \mathrm{H}\right), 7.54-7.49(\mathrm{~m}, 2 \mathrm{H}), 7.43-7.37(\mathrm{~m}, 4 \mathrm{H}), 6.55$ (dd, $J=3.6$ and $1.7 \mathrm{~Hz}, 1 \mathrm{H}), 6.30\left(\mathrm{~d}, J_{\mathrm{H}-\mathrm{F}}=47.9 \mathrm{~Hz}, 1 \mathrm{H}\right) ;{ }^{13} \mathrm{C} \mathrm{NMR}\left(100 \mathrm{MHz}, \mathrm{CDCl}_{3}\right): \delta 183.2(\mathrm{~d}$, $\left.J_{C-F}=23.9 \mathrm{~Hz}\right), 149.9,147.6,134.2\left(\mathrm{~d}, J_{C-F}=20.3 \mathrm{~Hz}\right), 129.7\left(\mathrm{~d}, J_{C-F}=2.4 \mathrm{~Hz}\right), 128.9,127.2\left(\mathrm{~d}, J_{C-F}\right.$ $=6.0 \mathrm{~Hz}), 120.6\left(\mathrm{~d}, J_{C-F}=6.8 \mathrm{~Hz}\right), 112.7,93.8\left(\mathrm{~d}, J_{C-F}=186.4 \mathrm{~Hz}\right) ;{ }^{19} \mathrm{~F} N M R\left(376 \mathrm{MHz}, \mathrm{CDCl}_{3}\right)$ : $\delta-181.0$ (d, $J=48.0 \mathrm{~Hz}$ ); IR (ATR): $v_{\max } 2928,1687,1568,1463,1393,1015,991,973,883,822$, $696,625 \mathrm{~cm}^{-1}$; ESI-HRMS m/z calcd for $\mathrm{C}_{12} \mathrm{H}_{10} \mathrm{FO}_{2}[\mathrm{M}+\mathrm{H}]^{+} 205.0659$, found 205.0664 . 


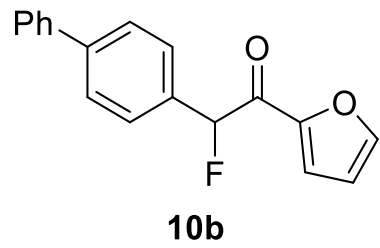

2-Fluoro-1-(furan-2-yl)-2-([1,1'-biphenyl]-4-yl)ethan-1-one 10b. Yield: 40\% (113 mg, 403 $\mu \mathrm{mol})$. Solvent system for flash column chromatography: petroleum ether/EtOAc: 90/10; White solid; $\mathrm{Mp}: 130{ }^{\circ} \mathrm{C} ;{ }^{1} \mathrm{H}$ NMR $\left(400 \mathrm{MHz}, \mathrm{CDCl}_{3}\right): \delta$ 7.65-7.55 $(\mathrm{m}, 7 \mathrm{H}), 7.46-7.33(\mathrm{~m}, 4 \mathrm{H})$, $6.57(\mathrm{dd}, J=3.7$ and $1.7 \mathrm{~Hz}, 1 \mathrm{H}), 6.35\left(\mathrm{~d}, \mathrm{~J}_{\mathrm{H}-\mathrm{F}}=47.9 \mathrm{~Hz}, 1 \mathrm{H}\right) ;{ }^{13} \mathrm{C} \mathrm{NMR}\left(100 \mathrm{MHz}, \mathrm{CDCl}_{3}\right): \delta 183.2$ $\left(\mathrm{d}, J_{C-F}=24.1 \mathrm{~Hz}\right), 149.9,147.7,142.7\left(\mathrm{~d}, J_{C-F}=2.4 \mathrm{~Hz}\right), 140.4,133.1\left(\mathrm{~d}, J_{C-F}=20.4 \mathrm{~Hz}\right), 129.0$, $127.8,127.7,127.6\left(d, J_{C-F}=5.8 \mathrm{~Hz}\right), 127.3,120.7\left(\mathrm{~d}, J_{C-F}=6.8 \mathrm{~Hz}\right), 112.8,93.6\left(\mathrm{~d}, J_{C-F}=186.2\right.$ $\mathrm{Hz}$ ); ${ }^{19} \mathrm{~F} \mathrm{NMR} \mathrm{(376} \mathrm{MHz,} \mathrm{CDCl} 3$ ): $\delta-180.7$ (d, $J=47.8 \mathrm{~Hz}$ ); IR (ATR): $v_{\max } 2359,1689,1569,1463$, 1395, 1330, 1165, 1128, 1074, 1023, 1016, 973, 884, 817, 768, 728, 691, $663 \mathrm{~cm}^{-1}$; ESI-HRMS $\mathrm{m} / \mathrm{z}$ calcd for $\mathrm{C}_{18} \mathrm{H}_{13} \mathrm{FO}_{2} \mathrm{Na}[\mathrm{M}+\mathrm{Na}]^{+} 303.0792$, found 303.0792 .

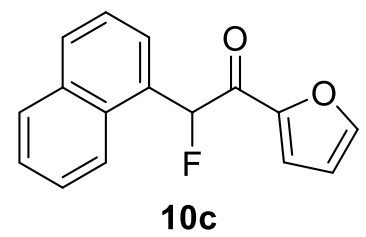

2-Fluoro-1-(furan-2-yl)-2-(naphthalen-1-yl)ethan-1-one 10c. Yield: 42\% (1.10 g, 4.3 mmol). Solvent system for flash column chromatography: petroleum ether/EtOAc: 90/10; White solid; Mp: $86^{\circ} \mathrm{C} ;{ }^{1} \mathrm{H} N M R\left(400 \mathrm{MHz}, \mathrm{CDCl}_{3}\right): \delta 8.29(\mathrm{~d}, J=8.4 \mathrm{~Hz}, 1 \mathrm{H}), 7.93-7.87(\mathrm{~m}, 2 \mathrm{H}), 7.66(\mathrm{dt}, J=$ 7.2 and $1.6 \mathrm{~Hz}, 1 \mathrm{H}), 7.63-7.45(\mathrm{~m}, 4 \mathrm{H}), 7.35(\mathrm{ddd}, J=3.6,1.5$ and $0.7 \mathrm{~Hz}, 1 \mathrm{H}), 6.94\left(\mathrm{~d}, J_{H-F}=47.4\right.$ $\mathrm{Hz}, 1 \mathrm{H}), 6.51(\mathrm{dd}, J=3.7$ and $1.7 \mathrm{~Hz}, 1 \mathrm{H}) ;{ }^{13} \mathrm{C} \mathrm{NMR}\left(100 \mathrm{MHz}, \mathrm{CDCl}_{3}\right): \delta 183.4\left(\mathrm{~d}, J_{C-F}=23.6 \mathrm{~Hz}\right)$, $150.2,147.5,134.1,131.2,130.9,130.2$ (d, $J_{C-F}=18.5 \mathrm{~Hz}$ ), 129.0, 127.5 (d, $J_{C-F}=7.6 \mathrm{~Hz}$ ), 127.3, 126.4, 125.2, 124.0, 120.4 (d, $\left.J_{C-F}=5.7 \mathrm{~Hz}\right), 112.6,92.5\left(\mathrm{~d}, J_{C-F}=185.5 \mathrm{~Hz}\right) ;{ }^{19} \mathrm{~F} \mathrm{NMR}(376 \mathrm{MHz}$, $\mathrm{CDCl}_{3}$ ): $\delta-181.7$ (d, J = 47.8 Hz); IR (ATR): $v_{\max } 2924,2853,1686,1567,1512,1462,1392,1258$, 1166, 1023, 970, 883, 827, 801, $772 \mathrm{~cm}^{-1}$; ESI-HRMS m/z calcd for $\mathrm{C}_{16} \mathrm{H}_{11} \mathrm{FO}_{2} \mathrm{Na}[\mathrm{M}+\mathrm{Na}]^{+}$ 277.0635 , found 277.0635 . 


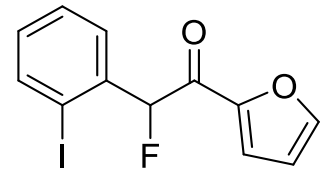

$10 \mathrm{~d}$

2-Fluoro-1-(furan-2-yl)-2-(2-iodophenyl)ethan-1-one 10d. Yield: 36\% (119 mg, $360 \mu \mathrm{mol}$ ). Solvent system for flash column chromatography: cyclohexane/EtOAc: 90/10; Yellow solid; Mp: $78^{\circ} \mathrm{C} ;{ }^{1} \mathrm{H} \mathrm{NMR}\left(400 \mathrm{MHz}, \mathrm{CDCl}_{3}\right): \delta 7.93(\mathrm{dt}, J=8.0$ and $1.0 \mathrm{~Hz}, 1 \mathrm{H}), 7.64(\mathrm{dd}, J=1.7$ and $0.7 \mathrm{~Hz}, 1 \mathrm{H}), 7.45-7.35(\mathrm{~m}, 3 \mathrm{H}), 7.10(\mathrm{tt}, J=7.6$ and $1.7 \mathrm{~Hz}, 1 \mathrm{H}), 6.65\left(\mathrm{~d}, J_{H-F}=46.7 \mathrm{~Hz}, 1 \mathrm{H}\right), 6.57$ $(\mathrm{dd}, J=3.7$ and $1.7 \mathrm{~Hz}, 1 \mathrm{H}) ;{ }^{13} \mathrm{C} \mathrm{NMR}\left(100 \mathrm{MHz}, \mathrm{CDCl}_{3}\right): \delta 182.2\left(\mathrm{~d}, J_{C-F}=23.7 \mathrm{~Hz}\right), 150.3,147.9$, 140.2, 137.2 (d, $\left.J_{C-F}=19.9 \mathrm{~Hz}\right), 131.6\left(\mathrm{~d}, J_{C-F}=2.8 \mathrm{~Hz}\right.$ ), $129.3\left(\mathrm{~d}, J_{C-F}=5.4 \mathrm{~Hz}\right), 129.0,120.5$ (d, $\left.J_{C-F}=4.8 \mathrm{~Hz}\right), 112.7,99.9\left(\mathrm{~d}, J_{C-F}=4.9 \mathrm{~Hz}\right), 96.0\left(\mathrm{~d}, J_{C-F}=185.2 \mathrm{~Hz}\right) ;{ }^{19} \mathrm{~F} N M R\left(376 \mathrm{MHz} \mathrm{CDCl}_{3}\right):$ $\delta-176.5$ (d, $J=46.8 \mathrm{~Hz}$ ); IR (ATR): $v_{\max } 1687,1567,1462,1393,1193,1086,1012,977,916$, 824, 758, $614 \mathrm{~cm}^{-1}$; ESI-HRMS m/z calcd for $\mathrm{C}_{12} \mathrm{H}_{9} \mathrm{FIO}_{2}[\mathrm{M}+\mathrm{H}]^{+} 330.9626$, found 330.9627.<smiles>O=C(c1ccco1)C(F)c1cccc(C(F)(F)F)c1</smiles>

2-Fluoro-1-(furan-2-yl)-2-(3-(trifluoromethyl)phenyl)ethan-1-one 10e. Yield: 41\% (111 mg, $408 \mu \mathrm{mol})$. Solvent system for flash column chromatography: petroleum ether/EtOAc: 90/10; Yellowish oil; ${ }^{1} \mathrm{H}$ NMR (400 MHz, $\mathrm{CDCl}_{3}$ ): $\delta 7.79(\mathrm{~s}, 1 \mathrm{H}), 7.71$ (d, $J=7.9 \mathrm{~Hz}, 1 \mathrm{H}$ ), 7.66 (obs. d, J = $1.1 \mathrm{~Hz}, 1 \mathrm{H}$ ), 7.65 (obs. d, $J=7.8 \mathrm{~Hz}, 1 \mathrm{H}$ ), 7.53 (app. t, $J=7.8 \mathrm{~Hz}, 1 \mathrm{H}$ ), 7.45 (dd, $J=3.7$ and 2.1 $\mathrm{Hz}, 1 \mathrm{H}), 6.59(\mathrm{dd}, J=3.7$ and $1.7 \mathrm{~Hz}, 1 \mathrm{H}), 6.35\left(\mathrm{~d}, \mathrm{~J}_{\mathrm{H}-F}=47.5 \mathrm{~Hz}, 1 \mathrm{H}\right) ;{ }^{13} \mathrm{C} \mathrm{NMR}\left(100 \mathrm{MHz}, \mathrm{CDCl}_{3}\right)$ : $\delta 182.4\left(\mathrm{~d}, J_{C-F}=23.8 \mathrm{~Hz}\right), 149.7,148.0,135.3\left(\mathrm{~d}, J_{C-F}=21.0 \mathrm{~Hz}\right), 131.5\left(\mathrm{~d}, J_{C-F}=32.7 \mathrm{~Hz}\right), 130.2$ (d, $\left.J_{C-F}=6.2 \mathrm{~Hz}\right), 129.6,126.5,123.9\left(\mathrm{~d}, J_{C-F}=272.7 \mathrm{~Hz}\right), 123.8\left(\mathrm{dq}, J_{C-F}=7.3\right.$ and $\left.3.6 \mathrm{~Hz}\right), 121.1$ (d, $\left.J_{C-F}=7.4 \mathrm{~Hz}\right), 112.9,93.0\left(\mathrm{~d}, J_{C-F}=188.0 \mathrm{~Hz}\right) ;{ }^{19} \mathrm{~F} \mathrm{NMR}\left(376 \mathrm{MHz}, \mathrm{CDCl}_{3}\right): \delta-62.8,-183.6(\mathrm{~d}$, $J=47.3 \mathrm{~Hz}$ ); IR (ATR): $v_{\max } 1689,1569,1463,1394,1329,1167,1126,1074,1016,884,768$, $700,663 \mathrm{~cm}^{-1}$; ESI-HRMS m/z calcd for $\mathrm{C}_{13} \mathrm{H}_{9} \mathrm{~F}_{4} \mathrm{O}_{2}[\mathrm{M}+\mathrm{H}]^{+} 273.0533$, found 273.0531 


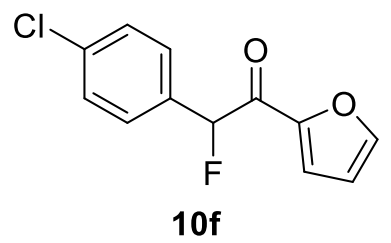

2-Fluoro-1-(furan-2-yl)-2-(4-chlorophenyl)ethan-1-one 10f. Yield: 47\% (111 mg, $465 \mu \mathrm{mol})$. Solvent system for flash column chromatography: petroleum ether/EtOAc: 90/10; White solid; Mp: $58^{\circ} \mathrm{C} ;{ }^{1} \mathrm{H} N M R\left(400 \mathrm{MHz}, \mathrm{CDCl}_{3}\right.$ ): $\delta 7.64$ (A of AMX syst., $J=1.2 \mathrm{~Hz}, 1 \mathrm{H}$ ), $7.45(\mathrm{~d}, J=9.3 \mathrm{~Hz}$, 2H), 7.40 (M of AMX syst., $J=3.6$ and $1.9 \mathrm{~Hz}, 1 \mathrm{H}$ ), $7.37(\mathrm{~d}, J=8.7 \mathrm{~Hz}, 2 \mathrm{H}$ ), 6.57 (X of AMX syst., $J=3.7$ and $1.7 \mathrm{~Hz}, 1 \mathrm{H}), 6.28\left(\mathrm{~d}, J_{H-F}=47.7 \mathrm{~Hz}, 1 \mathrm{H}\right) ;{ }^{13} \mathrm{C} \mathrm{NMR}\left(100 \mathrm{MHz}, \mathrm{CDCl}_{3}\right): \delta 182.8\left(\mathrm{~d}, J_{C-F}=\right.$ $23.9 \mathrm{~Hz}), 149.7,147.8,135.8\left(\mathrm{~d}, J_{C-F}=2.8 \mathrm{~Hz}\right), 132.7\left(\mathrm{~d}, J_{C-F}=20.9 \mathrm{~Hz}\right), 129.3,128.5\left(\mathrm{~d}, J_{C-F}=6.0\right.$ $\mathrm{Hz}), 120.8\left(\mathrm{~d}, J_{C-F}=6.8 \mathrm{~Hz}\right), 112.8,93.0\left(\mathrm{~d}, J_{C-F}=187.1 \mathrm{~Hz}\right) ;{ }^{19} \mathrm{~F} \mathrm{NMR}\left(376 \mathrm{MHz}, \mathrm{CDCl}_{3}\right): \delta-181.7$ (d, $J=47.7 \mathrm{~Hz}$ ); IR (ATR): $v_{\max } 2924,1686,1568,1492,1463,1393,1268,1090,1015,976,883$, 806, $764 \mathrm{~cm}^{-1}$; ESI-HRMS m/z calcd for $\mathrm{C}_{12} \mathrm{H}_{8}{ }^{35} \mathrm{ClFO}_{2} \mathrm{Na}[\mathrm{M}+\mathrm{Na}]^{+} 261.0089$, found 261.0086.

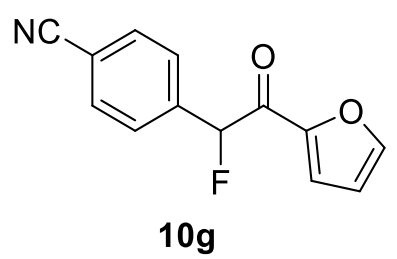

2-Fluoro-1-(furan-2-yl)-2-(4-cyanophenyl)ethan-1-one 10g. Yield: 37\% (80 mg, $373 \mu \mathrm{mol})$. Solvent system for flash column chromatography: petroleum ether/EtOAc: $95 / 5$ to 80/20; Offwhite solid; $\mathrm{Mp}: 50{ }^{\circ} \mathrm{C} ;{ }^{1} \mathrm{H}$ NMR $\left(300 \mathrm{MHz}, \mathrm{CDCl}_{3}\right): \delta 7.76-7.60(\mathrm{~m}, 5 \mathrm{H}), 7.50-7.41(\mathrm{~m}, 1 \mathrm{H}), 6.60$ (dd, $J=3.7$, and $1.7 \mathrm{~Hz}, 1 \mathrm{H}), 6.34(\mathrm{~d}, J=47.6 \mathrm{~Hz}, 1 \mathrm{H}) ;{ }^{13} \mathrm{C} \mathrm{NMR}\left(100 \mathrm{MHz}, \mathrm{CDCl}_{3}\right): \delta 182.1,181.9$, 149.6, 148.2, 139.2, 139.0, 132.7, 127.4, 127.3, 121.4, 121.3, 118.3, 113.6, 113.1, 93.8, 91.9; ${ }^{19} \mathrm{~F}$ NMR (376 MHz, CDCl ): $\delta-186.0$ (d, $J=47.4 \mathrm{~Hz}$ ); IR (ATR): $v_{\max } 2983,2961,2916,2877$, 2233, 1687, 1459, 1335, 1161, 1094, 1037, 1009, 838, 756, $692 \mathrm{~cm}^{-1}$; ESI-HRMS m/z calcd for $\mathrm{C}_{13} \mathrm{H}_{8} \mathrm{FNO}_{2} \mathrm{Na}[\mathrm{M}+\mathrm{Na}]^{+}$252.0431, found 252.0438. 


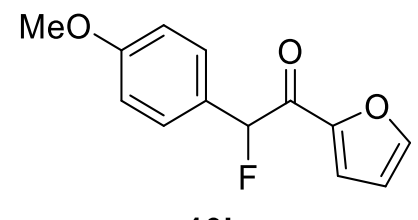

$10 \mathrm{~h}$

2-Fluoro-1-(furan-2-yl)-2-(4-methoxyphenyl)ethan-1-one 10h. Yield: 30\% (70 mg, 299 umol). Solvent system for flash column chromatography: petroleum ether/EtOAc: $95 / 5$ to $90 / 10$; Yellow oil; ${ }^{1} \mathrm{H}$ NMR $\left(400 \mathrm{MHz}, \mathrm{CDCl}_{3}\right): \delta 7.60(\mathrm{~d}, J=1.8 \mathrm{~Hz}, 1 \mathrm{H}), 7.43(\mathrm{~d}, J=1.7 \mathrm{~Hz}, 1 \mathrm{H}), 7.41(\mathrm{~d}$, $J=1.7 \mathrm{~Hz}, 1 \mathrm{H}), 7.35(\mathrm{dd}, J=3.7$ and $1.0 \mathrm{~Hz}, 1 \mathrm{H}), 6.91(\mathrm{~d}, J=8.0 \mathrm{~Hz}, 2 \mathrm{H}), 6.53(\mathrm{dd}, J=3.7$ and $1.7 \mathrm{~Hz}, 1 \mathrm{H}), 6.26\left(\mathrm{~d}, J_{H-F}=47.9 \mathrm{~Hz}, 1 \mathrm{H}\right), 3.79(\mathrm{~s}, 3 \mathrm{H}) ;{ }^{13} \mathrm{C} \mathrm{NMR}\left(100 \mathrm{MHz}, \mathrm{CDCl}_{3}\right): \delta 183.4\left(\mathrm{~d}, J_{C-F}\right.$ $=24.8 \mathrm{~Hz}$ ), 160.8 (d, $\left.J_{C-F}=2.6 \mathrm{~Hz}\right), 150.1,147.5,129.2\left(\mathrm{~d}, J_{C-F}=5.2 \mathrm{~Hz}\right), 126.3\left(\mathrm{~d}, J_{C-F}=21.0 \mathrm{~Hz}\right)$, $120.3\left(\mathrm{~d}, J_{C-F}=6.1 \mathrm{~Hz}\right), 114.5,112.7,93.3\left(\mathrm{~d}, J_{C-F}=185.4 \mathrm{~Hz}\right), 55.4 ;{ }^{19} \mathrm{~F} \mathrm{NMR}\left(376 \mathrm{MHz}, \mathrm{CDCl}_{3}\right)$ : $\delta$-176.4 (d, $J=47.9 \mathrm{~Hz}$ ); IR (ATR): $v_{\max } 2934,2838,1672,1600,1510,1463,1250,1175,1082$, $820,768 \mathrm{~cm}^{-1}$; ESI-HRMS m/z calcd for $\mathrm{C}_{13} \mathrm{H}_{12} \mathrm{FO}_{3}[\mathrm{M}+\mathrm{H}]^{+} 235.0765$, found 235.0765.

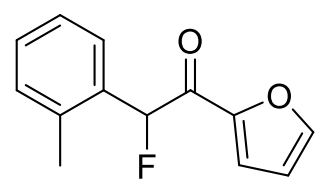

$10 \mathrm{j}$

2-Fluoro-1-(furan-2-yl)-2-(o-tolyl)ethan-1-one 10j. Yield: 44\% (95 mg, $435 \mu \mathrm{mol})$. Solvent system for flash column chromatography: petroleum ether/EtOAc: 95/5; Yellowish solid; Mp: $32{ }^{\circ} \mathrm{C} ;{ }^{1} \mathrm{H} \mathrm{NMR}\left(400 \mathrm{MHz}, \mathrm{CDCl}_{3}\right): \delta 7.60(\mathrm{dd}, J=1.7$ and $0.7 \mathrm{~Hz}, 1 \mathrm{H}), 7.38(\mathrm{tt}, J=7.5$ and $1.5 \mathrm{~Hz}$, $1 \mathrm{H}), 7.36(\mathrm{dd}, J=3.7$ and $1.8 \mathrm{~Hz}, 1 \mathrm{H}), 7.31-7.26(\mathrm{~m}, 1 \mathrm{H}), 7.25-7.18(\mathrm{~m}, 2 \mathrm{H}), 6.49\left(\mathrm{~d}, J_{H-F}=47.7\right.$ $\mathrm{Hz}, 1 \mathrm{H}$ ), 6.54 (dd, $J=3.7$ and $1.7 \mathrm{~Hz}, 1 \mathrm{H}), 2.51$ (app. d, $J=1.7 \mathrm{~Hz}, 3 \mathrm{H}) ;{ }^{13} \mathrm{C} \mathrm{NMR}(100 \mathrm{MHz}$, $\left.\mathrm{CDCl}_{3}\right): \delta 183.7\left(\mathrm{~d}, J_{C-F}=24.1 \mathrm{~Hz}\right), 150.3,147.5,137.6\left(\mathrm{~d}, J_{C-F}=3.5 \mathrm{~Hz}\right), 132.7\left(\mathrm{~d}, J_{C-F}=18.7 \mathrm{~Hz}\right)$, 131.3, $129.9\left(\mathrm{~d}, J_{C-F}=3.1 \mathrm{~Hz}\right), 128.3\left(\mathrm{~d}, J_{C-F}=6.0 \mathrm{~Hz}\right), 126.5,120.3\left(\mathrm{~d}, J_{C-F}=6.6 \mathrm{~Hz}\right), 112.7,91.7$ $\left(\mathrm{d}, J_{C-F}=184.4 \mathrm{~Hz}\right), 19.5 ;{ }^{19} \mathrm{~F} N M R\left(376 \mathrm{MHz}, \mathrm{CDCl}_{3}\right): \delta-179.2(\mathrm{~d}, J=47.3 \mathrm{~Hz}) ; \mathrm{IR}($ ATR $): v_{\max }$ 2924, 1689, 1568, 1462, 1392, 1269, 1164, 1014, 883, 829, $760 \mathrm{~cm}^{-1}$; ESI-HRMS m/z calcd for $\mathrm{C}_{13} \mathrm{H}_{11} \mathrm{FO}_{2} \mathrm{Na}[\mathrm{M}+\mathrm{Na}]^{+} 241.0635$, found 241.0633 . 


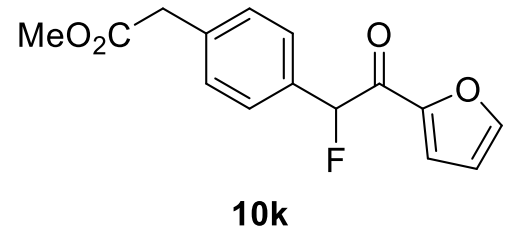

Methyl 2-Fluoro-1-(furan-2-yl)-4-(oxoethylphenyl)ethan-1-one 10k.Yield: 38\% (105 mg, 380 $\mu \mathrm{mol})$. Solvent system for flash column chromatography: petroleum ether/EtOAc: 80/20; Offwhite solid; $\mathrm{Mp}: 83^{\circ} \mathrm{C} ;{ }^{1} \mathrm{H}$ NMR $\left(400 \mathrm{MHz}, \mathrm{CDCl}_{3}\right): \delta 7.63-7.61(\mathrm{~m}, 1 \mathrm{H}), 7.47(\mathrm{~d}, J=8.1 \mathrm{~Hz}, 2 \mathrm{H})$, 7.40-7.37 (m, 1H), 7.31 (d, J = 7.7 Hz, 2H), 6.56-6.53 (m, 1H), $6.29\left(\mathrm{~d}, J_{H-F}=47.7 \mathrm{~Hz}, 1 \mathrm{H}\right)$; 3.693.67 (app. m, 3H), 3.62 (s, 2H); ${ }^{13} \mathrm{C} \mathrm{NMR} \mathrm{(100} \mathrm{MHz,} \mathrm{CDCl} 3$ ): $\delta 183.1$ (d, $J_{C-F}=24.1 \mathrm{~Hz}$ ), 171.7, 149.9, 147.7, $135.6\left(\mathrm{~d}, J_{C-F}=2.4 \mathrm{~Hz}\right), 133.1\left(\mathrm{~d}, J_{C-F}=20.5 \mathrm{~Hz}\right), 130.0,127.5$ (d, $J_{C-F}=6.0 \mathrm{~Hz}$ ), $120.7\left(\mathrm{~d}, J_{C-F}=6.8 \mathrm{~Hz}\right), 112.7,93.5\left(\mathrm{~d}, J_{C-F}=186.3 \mathrm{~Hz}\right), 52.3,41.0 ;{ }^{19} \mathrm{~F} \mathrm{NMR}\left(376 \mathrm{MHz}, \mathrm{CDCl}_{3}\right): \delta$ -180.9 (app. dd, $J=47.7$ and $21.5 \mathrm{~Hz}$ ); IR (ATR): $v_{\max } \mathrm{Cm}^{-1}$; 2950, 1730, 1684, 1468, 1294, 1259, $1132,1088,1026,1014,992,822,803,762 \mathrm{~cm}^{-1}$; ESI-HRMS m/z calcd for $\mathrm{C}_{15} \mathrm{H}_{17} \mathrm{FNO}_{4}\left[\mathrm{M}+\mathrm{NH}_{4}\right]^{+}$ 294.1136, found 294.1139 .

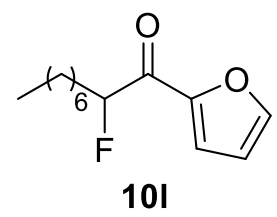

2-Fluoro-1-(furan-2-yl)nonan-1-one 10l. Yield: Isolation of $76 \mathrm{mg}$ of the fluorinated product (30\%) contaminated with the corresponding protonated byproduct (4\%). Solvent system for flash column chromatography: petroleum ether/EtOAc: 98/2; Yellow oil; ${ }^{1} \mathrm{H} N M R(400 \mathrm{MHz}$, $\left.\mathrm{CDCl}_{3}\right): \delta 7.66(\mathrm{~d}, J=1.7 \mathrm{~Hz}, 1 \mathrm{H}), 7.41(\mathrm{t}, J=3.0 \mathrm{~Hz}, 1 \mathrm{H}), 6.58(\mathrm{dd}, J=3.6$ and $1.7 \mathrm{~Hz}, 1 \mathrm{H}), 5.30$ (ddd, $J_{H-F}=49.6,7.4$ and $\left.4.8 \mathrm{~Hz}, 1 \mathrm{H}\right), 2.01-1.88(\mathrm{~m}, 2 \mathrm{H}), 1.54-1.47(\mathrm{~m}, 2 \mathrm{H}), 1.36-1.23(\mathrm{~m}, 8 \mathrm{H})$, $0.87(\mathrm{t}, J=6.9 \mathrm{~Hz}, 3 \mathrm{H}) ;{ }^{13} \mathrm{C} \mathrm{NMR}\left(100 \mathrm{MHz}, \mathrm{CDCl}_{3}\right): \delta 186.1\left(\mathrm{~d}, J_{C-F}=21.9 \mathrm{~Hz}\right), 150.1\left(\mathrm{~d}, J_{C-F}=1.5\right.$ $\mathrm{Hz}), 147.6,120.8\left(\mathrm{~d}, J_{C-F}=9.6 \mathrm{~Hz}\right), 112.6,94.4\left(\mathrm{~d}, J_{C-F}=184.0 \mathrm{~Hz}\right), 33.0\left(\mathrm{~d}, J_{C-F}=21.0 \mathrm{~Hz}\right), 31.8$, 29.2, 29.1, 24.7 (d, $J_{C-F}=3.2 \mathrm{~Hz}$ ), 22.7, 14.2; ${ }^{19} \mathrm{~F} \mathrm{NMR} \mathrm{(376} \mathrm{MHz,} \mathrm{CDCl}_{3}$ ): $\delta$-191.7 (app. dt, $J=$ 50.7 and $26.6 \mathrm{~Hz}$ ); IR (ATR): $v_{\max } 2956,2927,2857,1673,1568,1464,1394,1081,1019,884$, $768,654 \mathrm{~cm}^{-1}$; ESI-HRMS m/z calcd for $\mathrm{C}_{13} \mathrm{H}_{20} \mathrm{FO}_{2}[\mathrm{M}+\mathrm{H}]^{+} 227.1442$, found 227.1447. 


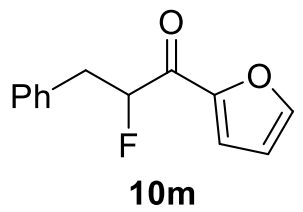

2-Fluoro-1-(furan-2-yl)-3-phenylpropan-1-one 10m. Yield: 38\% (82 mg, $376 \mu \mathrm{mol})$. Solvent system for flash column chromatography: cyclohexane/EtOAc: 90/10; Brown oil; ${ }^{1} \mathrm{H}$ NMR (400 $\left.\mathrm{MHz}, \mathrm{CDCl}_{3}\right): \delta 7.66(\mathrm{~d}, J=1.7 \mathrm{~Hz}, 1 \mathrm{H}), 7.37-7.25(\mathrm{~m}, 6 \mathrm{H}), 6.56(\mathrm{dd}, J=3.7$ and $1.7 \mathrm{~Hz}, 1 \mathrm{H}), 5.49$ (ddd, $J_{H-F}=49.1,8.3$ and $\left.3.8 \mathrm{~Hz}, 1 \mathrm{H}\right), 3.40-3.15(\mathrm{~m}, 2 \mathrm{H}) ;{ }^{13} \mathrm{C} \mathrm{NMR}\left(100 \mathrm{MHz}, \mathrm{CDCl}_{3}\right): \delta 184.9(\mathrm{~d}$, $\left.J_{C-F}=22.2 \mathrm{~Hz}\right), 150.1,147.8,135.6,129.6,128.7,127.3,121.2\left(d, J_{C-F}=9.6 \mathrm{~Hz}\right), 112.7,94.3(\mathrm{~d}$, $J_{C-F}=187.2 \mathrm{~Hz}$ ), $39.1\left(\mathrm{~d}, J_{C-F}=21.1 \mathrm{~Hz}\right) ;{ }^{19} \mathrm{~F} \mathrm{NMR}\left(376 \mathrm{MHz}, \mathrm{CDCl}_{3}\right): \delta-189.4$ (ddd, $J=52.1,31.4$ and $23.2 \mathrm{~Hz}$ ); IR (ATR): $v_{\max } 1672,1567,1497,1463,1394,1300,1274,1164,1080,1025,987$, $883,852,766,752,699,662 \mathrm{~cm}^{-1}$; ESI-HRMS m/z calcd for $\mathrm{C}_{13} \mathrm{H}_{12} \mathrm{FO}_{2}[\mathrm{M}+\mathrm{H}]^{+}$219.0816, found 219.0814 .

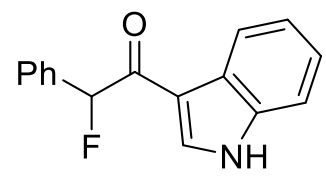

11

2-Fluoro-1-(1H-indol-3-yl)-2-phenylethan-1-one 11. Yield: 45\% (114 mg, $450 \mu \mathrm{mol})$. Solvent system for flash column chromatography: petroleum ether/EtOAc: 90/10; White solid; Mp: $169{ }^{\circ} \mathrm{C}$ (degradation); ${ }^{1} \mathrm{H}$ NMR $\left(400 \mathrm{MHz}, \mathrm{CD}_{3} \mathrm{OD}\right){ }^{59}: \delta 8.28-8.25(\mathrm{~m}, 2 \mathrm{H}), 7.57-7.54(\mathrm{~m}, 2 \mathrm{H})$, 7.46-7.42 (m, 1H), 7.40-7.33 (m, 3H), 7.26-7.19 (m, 2H), $6.44\left(\mathrm{~d}, J_{H-F}=48.3 \mathrm{~Hz}, 1 \mathrm{H}\right) ;{ }^{13} \mathrm{C} N M R$ $\left(100 \mathrm{MHz}, \mathrm{CD}_{3} \mathrm{OD}\right): \delta 192.4\left(\mathrm{~d}, J_{C-F}=22.4 \mathrm{~Hz}\right), 138.0,137.7\left(\mathrm{~d}, J_{C-F}=20.1 \mathrm{~Hz}\right), 136.0\left(\mathrm{~d}, J_{C-F}=9.3\right.$ $\mathrm{Hz}), 130.3\left(\mathrm{~d}, J_{C-F}=2.6 \mathrm{~Hz}\right), 129.8,128.3\left(\mathrm{~d}, J_{C-F}=5.8 \mathrm{~Hz}\right), 127.5,124.8,123.7,122.9,114.6$, 113.0, 95.4 (d, $\left.J_{C-F}=182.6 \mathrm{~Hz}\right) ;{ }^{19} \mathrm{~F}$ NMR $\left(376 \mathrm{MHz}, \mathrm{CD}_{3} \mathrm{OD}\right.$ ): $\delta-190.8$ (d, J = $48.8 \mathrm{~Hz}$ ); IR (ATR): $v_{\max } 3247,2928,2355,1639,1582,1519,1495,1459,1427,1330,1244,1133,1026,940,823$, 750, $697 \mathrm{~cm}^{-1}$; ESI-HRMS m/z calcd for $\mathrm{C}_{16} \mathrm{H}_{13} \mathrm{FNO}[\mathrm{M}+\mathrm{H}]^{+}$254.0976, found 254.0971.

\footnotetext{
s9 The labile proton of the indole could not be observed.
} 


\section{Experimental Procedures and Characterization Data: \\ $\alpha$-Fluoroesters}

\section{General procedure:}

To a solution of amide (1.0 mmol) and 2-bromopyridine $(210 \mu \mathrm{L}, 2.2 \mathrm{mmol})$ in dry dichloromethane $(3 \mathrm{~mL})$ at $0{ }^{\circ} \mathrm{C}$ was added dropwise triflic anhydride $(250 \mu \mathrm{L}, 1.5 \mathrm{mmol})$. The resulting brown solution was stirred for 5 minutes at $0{ }^{\circ} \mathrm{C}$ then $\mathrm{N}$-fluorobenzenesulfonimide $(631 \mathrm{mg}, 2.0 \mathrm{mmol})$ and the nucleophile $(2.0 \mathrm{mmol})$ were successively added in one portion. The orange reaction mixture was stirred at rt overnight and stopped by addition of a $1 \mathrm{M}$ solution of $\mathrm{NaOH}(5 \mathrm{~mL})$. The biphasic mixture was vigorously stirred for 2 hours then diluted with dichloromethane. The organic phase was washed with a $1 \mathrm{M}$ solution of $\mathrm{NaOH}(10 \mathrm{~mL})$, with a $1 \mathrm{M}$ solution of $\mathrm{HCl}(10 \mathrm{~mL})$, dried over $\mathrm{MgSO}_{4}$, filtered and concentrated under reduced pressure. The crude residue was finally purified by flash column chromatography over silica gel to yield the desired fluorinated ester.<smiles>CCOC(=O)C(F)c1ccccc1</smiles>

$12 \mathrm{a}$

Ethyl 2-fluoro-2-phenylacetate 12a. Yield: Isolation of $99 \mathrm{mg}$ of the fluorinated product (51\%) contaminated with the corresponding protonated byproduct (3\%). Solvent system for flash column chromatography: cyclohexane/EtOAc: 99/1; Colorless oil; ${ }^{1} \mathrm{H} \mathrm{NMR}\left(400 \mathrm{MHz}, \mathrm{CDCl}_{3}\right)$ : d 7.49-7.45 (m, 2H), 7.43-7.39 (m, 3H), $5.77\left(\mathrm{~d}, J_{H-F}=47.8 \mathrm{~Hz}, 1 \mathrm{H}\right), 4.32-4.17(\mathrm{~m}, 2 \mathrm{H}), 1.26(\mathrm{t}, J$ $=7.1 \mathrm{~Hz}, 3 \mathrm{H}) ;{ }^{13} \mathrm{C} N M R\left(100 \mathrm{MHz}, \mathrm{CDCl}_{3}\right): \delta 168.6\left(\mathrm{~d}, J_{C-F}=27.5 \mathrm{~Hz}\right), 134.4\left(\mathrm{~d}, J_{C-F}=20.5 \mathrm{~Hz}\right)$, $129.7\left(\mathrm{~d}, J_{C-F}=2.2 \mathrm{~Hz}\right), 128.8,126.7\left(\mathrm{~d}, J_{C-F}=6.1 \mathrm{~Hz}\right), 89.5\left(\mathrm{~d}, J_{C-F}=185.4 \mathrm{~Hz}\right), 61.9,14.1 ;{ }^{19} \mathrm{~F}$ NMR (376 MHz, $\mathrm{CDCl}_{3}$ ): $\delta-179.7(\mathrm{~d}, J=47.7 \mathrm{~Hz})$. This compound has been previously reported. ${ }^{\mathrm{S} 10}$

\footnotetext{
s10 Sheng, J.; Ni, H-Q.; Liu, G.; Li, Y.; Wang, X-S. Org. Lett. 2017, 19, 4480.
} 


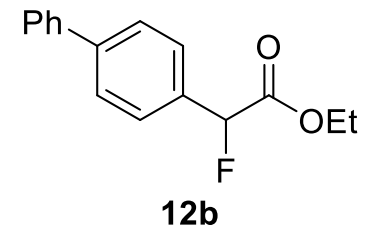

Ethyl 2-fluoro-2-([1,1'-biphenyl]-4-yl)acetate 12b. Yield: Isolation of $153 \mathrm{mg}$ of the fluorinated product (52\%) contaminated with the corresponding protonated byproduct (7\%). Solvent system for flash column chromatography: petroleum ether/EtOAc: 99/1; Yellow oil; ${ }^{1} \mathrm{H}$ NMR (400 MHz, $\left.\mathrm{CDCl}_{3}\right): \delta$ 7.66-7.52 (m, 6H), 7.48-7.43 (m, 2H), 7.40-7.35 (m, 1H), $5.82\left(\mathrm{~d}, J_{H-F}=47.8\right.$ $\mathrm{Hz}, 1 \mathrm{H}), 4.36-4.20(\mathrm{~m}, 2 \mathrm{H}), 1.29(\mathrm{t}, J=7.1 \mathrm{~Hz}, 3 \mathrm{H}) ;{ }^{13} \mathrm{C} \mathrm{NMR}\left(100 \mathrm{MHz}, \mathrm{CDCl}_{3}\right): \delta 168.7\left(\mathrm{~d}, J_{\mathrm{C}-\mathrm{F}}=\right.$ $27.6 \mathrm{~Hz}), 142.7\left(\mathrm{~d}, J_{C-F}=2.4 \mathrm{~Hz}\right), 140.4,133.3\left(\mathrm{~d}, J_{C-F}=20.6 \mathrm{~Hz}\right), 129.0,127.8,127.6,127.3$, $127.2,89.3\left(\mathrm{~d}, J_{C-F}=185.5 \mathrm{~Hz}\right), 62.0,14.2 ;{ }^{19} \mathrm{~F} \mathrm{NMR}\left(376 \mathrm{MHz}, \mathrm{CDCl}_{3}\right): \delta-179.6(\mathrm{~d}, J=47.7 \mathrm{~Hz})$; IR (ATR): $v_{\max } 3059,3031,2981,1757,1487,1277,1216,1188,1055,842,759,736,698 \mathrm{~cm}^{-1}$; ESI-HRMS m/z calcd for $\mathrm{C}_{16} \mathrm{H}_{19} \mathrm{FNO}_{2}\left[\mathrm{M}+\mathrm{NH}_{4}\right]^{+} 276,1394$, found 276,1393.<smiles>CCOC(=O)C(F)c1cccc2ccccc12</smiles>

Ethyl 2-fluoro-2-(naphthalen-1-yl)acetate 12c. Yield: Isolation of $66 \mathrm{mg}$ of the fluorinated product $(55 \%)$ contaminated with the corresponding protonated byproduct (2\%). Solvent system for flash column chromatography: cyclohexane/EtOAc: 97/3; Yellow oil; ${ }^{1} \mathrm{H}$ NMR (400 $\left.\mathrm{MHz}, \mathrm{CDCl}_{3}\right): \delta 8.19(\mathrm{~d}, J=8.0 \mathrm{~Hz}, 1 \mathrm{H}), 7.90(\mathrm{t}, J=7.8 \mathrm{~Hz}, 2 \mathrm{H}), 7.62-7.46(\mathrm{~m}, 4 \mathrm{H}), 6.35\left(\mathrm{~d}, J_{H-F}=\right.$ $47.1 \mathrm{~Hz}, 1 \mathrm{H}$ ), 4.28 ( $\mathrm{A}$ of $A B X_{3}$ syst., $J=10.8$ and $7.1 \mathrm{~Hz}, 1 \mathrm{H}$ ), 4.18 (B of $A B X_{3}$ syst., $J=10.8$ and $7.1 \mathrm{~Hz}, 1 \mathrm{H}$ ), 1.20 (X of $\mathrm{ABX}_{3}$ syst., $\left.J=7.1 \mathrm{~Hz}, 3 \mathrm{H}\right) ;{ }^{13} \mathrm{C} \mathrm{NMR}\left(100 \mathrm{MHz}, \mathrm{CDCl}_{3}\right): \delta 169.0$ (d, $J_{C-F}=$ $27.5 \mathrm{~Hz}$ ), 133.9, 130.7, $130.6\left(\mathrm{~d}, J_{C-F}=2.7 \mathrm{~Hz}\right), 130.3\left(\mathrm{~d}, J_{C-F}=18.6 \mathrm{~Hz}\right), 128.9,127.1,126.9(\mathrm{~d}$, $\left.J_{C-F}=7.9 \mathrm{~Hz}\right), 126.3,125.2,123.8,88.7\left(\mathrm{~d}, J_{C-F}=185.4 \mathrm{~Hz}\right), 62.0,14.1 ;{ }^{19} \mathrm{~F} \mathrm{NMR}\left(376 \mathrm{MHz}, \mathrm{CDCl}_{3}\right)$ : $\delta-177.8(\mathrm{~d}, J=47.2 \mathrm{~Hz})$. This compound has been previously reported. ${ }^{\text {S11 }}$

\footnotetext{
S11 Wu, Y.; Zhang, H-R.; Cao, Y-X.; Lan, Q.; Wang, X-S. Org. Lett. 2016, 18, 5564.
} 


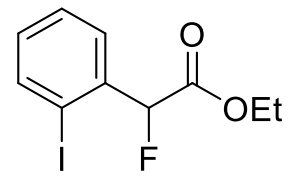

$12 d$

Ethyl 2-fluoro-2-(2-iodophenyl)acetate 12d. Yield: Isolation of $206 \mathrm{mg}$ of the fluorinated product $(63 \%)$ contaminated with the corresponding protonated byproduct $(4 \%)$. Solvent system for flash column chromatography: cyclohexane/EtOAc: 99/1; Colorless oil; ${ }^{1} \mathrm{H}$ NMR (400 MHz, $\mathrm{CDCl}_{3}$ ): $\delta 7.88$ (dt, $J=8.0$ and $1.0 \mathrm{~Hz}, 1 \mathrm{H}$ ), 7.46 (dd, $J=7.8$ and $1.7 \mathrm{~Hz}, 1 \mathrm{H}$ ), 7.39 (t, $J$ $=7.5 \mathrm{~Hz}, 1 \mathrm{H}), 7.08(\mathrm{tt}, J=7.6$ and $1.6 \mathrm{~Hz}, 1 \mathrm{H}), 6.07\left(\mathrm{~d}, J_{\mathrm{H}-\mathrm{F}}=46.5 \mathrm{~Hz}, 1 \mathrm{H}\right), 4.36-4.12(\mathrm{~m}, 2 \mathrm{H})$, $1.27(\mathrm{t}, J=7.1 \mathrm{~Hz}, 3 \mathrm{H}) ;{ }^{13} \mathrm{C} \mathrm{NMR}\left(100 \mathrm{MHz}, \mathrm{CDCl}_{3}\right): \delta 168.2\left(\mathrm{~d}, J_{C-F}=27.9 \mathrm{~Hz}\right), 140.0,137.5$ (d, $J_{C-F}=20.3 \mathrm{~Hz}$ ), $131.3\left(\mathrm{~d}, J_{C-F}=2.4 \mathrm{~Hz}\right), 128.8,128.2\left(\mathrm{~d}, J_{C-F}=6.6 \mathrm{~Hz}\right.$ ) $98.4\left(\mathrm{~d}, J_{C-F}=4.9 \mathrm{~Hz}\right), 92.5$ $\left(\mathrm{d}, J_{C-F}=185.6 \mathrm{~Hz}\right), 62.1,14.1 ;{ }^{19} \mathrm{~F}$ NMR $\left(376 \mathrm{MHz}, \mathrm{CDCl}_{3}\right): \delta-185.3$ (d, $J=46.4 \mathrm{~Hz}$ ); IR (ATR): $V_{\max } 1757,1469,1438,1370,1273,1213,1191,1118,1062,1013,682 \mathrm{~cm}^{-1}$; ESI-HRMS m/z calcd for $\mathrm{C}_{10} \mathrm{H}_{11} \mathrm{FIO}_{2}[\mathrm{M}+\mathrm{H}]^{+} 308.9782$, found 308.9790 .<smiles>CCOC(=O)C(F)c1ccc(Cl)cc1</smiles>

Ethyl 2-fluoro-2-(4-chlorophenyl)acetate 12e. Yield: 68\% (148 mg, $683 \mu \mathrm{mol})$. Solvent system for flash column chromatography: cyclohexane/EtOAc: 99/1; Yellow oil; ${ }^{1} \mathrm{H}$ NMR (400 MHz, $\left.\mathrm{CDCl}_{3}\right): \delta 7.45-7.35(\mathrm{~m}, 4 \mathrm{H}), 5.75\left(\mathrm{~d}, J_{\mathrm{H}-\mathrm{F}}=47.4 \mathrm{~Hz}, 1 \mathrm{H}\right), 4.32-4.17(\mathrm{~m}, 2 \mathrm{H}), 1.26(\mathrm{t}, J=7.2 \mathrm{~Hz}$, $3 \mathrm{H}) ;{ }^{13} \mathrm{C} \mathrm{NMR}\left(100 \mathrm{MHz}, \mathrm{CDCl}_{3}\right): \delta 168.3\left(\mathrm{~d}, J_{C-F}=27.4 \mathrm{~Hz}\right), 135.8,132.9\left(\mathrm{~d}, J_{C-F}=20.9 \mathrm{~Hz}\right), 129.2$, $128.1\left(\mathrm{~d}, J_{C-F}=6.2 \mathrm{~Hz}\right), 88.8\left(\mathrm{~d}, J_{C-F}=186.4 \mathrm{~Hz}\right), 62.2,14.2 ;{ }^{19} \mathrm{~F} \mathrm{NMR}\left(376 \mathrm{MHz}, \mathrm{CDCl}_{3}\right): \delta-180.8$ $\left(d, J=47.7 \mathrm{~Hz}\right.$ ). This compound has been previously reported. ${ }^{\mathrm{S} 12}$

\footnotetext{
S12 Fahandej-Sadi, A.; Lundgren, R.J. Synlett 2017, 28, 2886.
} 
<smiles>CCOC(=O)C(F)c1cccc(Cl)c1</smiles>

Ethyl 2-fluoro-2-(3-chlorophenyl)acetate 12f. Yield: Isolation of $177 \mathrm{mg}$ of the fluorinated product $(74 \%)$ contaminated with the corresponding protonated byproduct (8\%). Solvent system for flash column chromatography: cyclohexane/EtOAc: 99/1; Colorless oil; ${ }^{1} \mathrm{H}$ NMR (400 MHz, $\mathrm{CDCl}_{3}$ ): $\delta 7.48$ (app s, $\left.1 \mathrm{H}\right), 7.40-7.33(\mathrm{~m}, 3 \mathrm{H}), 5.74\left(\mathrm{~d}, J_{H-F}=47.5 \mathrm{~Hz}, 1 \mathrm{H}\right), 4.33-4.20$ $(\mathrm{m}, 2 \mathrm{H}), 1.27$ (t, J = 7.1 Hz, 3H); ${ }^{13} \mathrm{C} \mathrm{NMR}\left(100 \mathrm{MHz}, \mathrm{CDCl}_{3}\right): \delta 168.0$ (d, $J_{\mathrm{C}-F}=27.0 \mathrm{~Hz}$ ), 136.3 (d, $\left.J_{C-F}=21.0 \mathrm{~Hz}\right), 134.9,130.2,129.9,126.8\left(\mathrm{~d}, J_{C-F}=6.9 \mathrm{~Hz}\right), 124.7\left(\mathrm{~d}, J_{C-F}=6.4 \mathrm{~Hz}\right), 88.7\left(\mathrm{~d}, J_{C-F}=\right.$ 187.3 Hz), 62.2, 14.2; $\left.{ }^{19} \mathrm{~F} \mathrm{NMR} \mathrm{(376} \mathrm{MHz,} \mathrm{CDCl}_{3}\right): \delta-182.2(\mathrm{~d}, J=47.4 \mathrm{~Hz}$ ). This compound has been previously reported. ${ }^{\mathrm{S} 11}$<smiles>CCOC(=O)C(F)c1ccc(OC)cc1</smiles>

$12 \mathrm{~g}$

Ethyl 2-fluoro-2-(4-methoxyphenyl)acetate 12g. Yield: Isolation of $112 \mathrm{mg}$ of the fluorinated product $(49 \%)$ contaminated with the corresponding protonated byproduct $(4 \%)$. Solvent system for flash column chromatography: petroleum ether/EtOAc: 99/1; Yellowish oil; ${ }^{1} \mathrm{H}$ $\operatorname{NMR}\left(400 \mathrm{MHz}, \mathrm{CDCl}_{3}\right): \delta 7.40(\mathrm{~d}, J=1.5 \mathrm{~Hz}, 1 \mathrm{H}), 7.37(\mathrm{~d}, J=1.7 \mathrm{~Hz}, 1 \mathrm{H}), 6.92(\mathrm{~d}, J=8.5 \mathrm{~Hz}$, $2 \mathrm{H}), 5.71\left(\mathrm{~d}, J_{H-F}=47.9 \mathrm{~Hz}, 1 \mathrm{H}\right), 4.32-4.16(\mathrm{~m}, 2 \mathrm{H}), 3.82(\mathrm{~s}, 3 \mathrm{H}), 1.26(\mathrm{t}, J=7.1 \mathrm{~Hz}, 3 \mathrm{H}) ;{ }^{13} \mathrm{C} \mathrm{NMR}$ $\left(100 \mathrm{MHz}, \mathrm{CDCl}_{3}\right): \delta 169.0\left(\mathrm{~d}, J_{C-F}=28.4 \mathrm{~Hz}\right), 160.8\left(\mathrm{~d}, J_{C-F}=2.3 \mathrm{~Hz}\right), 128.7\left(\mathrm{~d}, J_{C-F}=5.3 \mathrm{~Hz}\right.$ ), 126.6 (d, $\left.J_{C-F}=21.1 \mathrm{~Hz}\right), 114.3,89.3\left(\mathrm{~d}, J_{C-F}=184.5 \mathrm{~Hz}\right), 61.9,55.5,14.2 ;{ }^{19} \mathrm{~F} \mathrm{NMR}\left(376 \mathrm{MHz}, \mathrm{CDCl}_{3}\right)$ : $\delta-174.7(\mathrm{~d}, J=47.8 \mathrm{~Hz})$. This compound has been previously reported. ${ }^{\mathrm{s} 13}$

\footnotetext{
s13 Su, Y-M.; Feng, G-S.; Wang, Z-Y.; Lan, Q.; Wang, X-S.; Angew. Chem. Int. Ed. 2015, 54, 6003.
} 
<smiles>COC(=O)C(F)c1cccc2ccccc12</smiles>

$12 \mathbf{i}$

Methyl 2-fluoro-2-(naphthalen-1-yl)acetate 12i. Yield: 65\% (142 mg, 651 umol). Solvent system for flash column chromatography: cyclohexane/EtOAc: 99/1; Yellowish oil; ${ }^{1} \mathrm{H}$ NMR $\left(400 \mathrm{MHz}, \mathrm{CDCl}_{3}\right): \delta 8.18(\mathrm{~d}, J=8.4 \mathrm{~Hz}, 1 \mathrm{H}), 7.91(\mathrm{t}, J=7.9 \mathrm{~Hz}, 2 \mathrm{H}), 7.63-7.47(\mathrm{~m}, 4 \mathrm{H}), 6.38(\mathrm{~d}$, $\left.J_{H-F}=46.8 \mathrm{~Hz}, 1 \mathrm{H}\right), 3.77(\mathrm{~s}, 3 \mathrm{H}) ;{ }^{13} \mathrm{C} N M R\left(100 \mathrm{MHz}, \mathrm{CDCl}_{3}\right): \delta 169.5\left(\mathrm{~d}, J_{C-F}=27.7 \mathrm{~Hz}\right), 134.0$, $130.8,130.8,130.2\left(\mathrm{~d}, J_{C-F}=18.7 \mathrm{~Hz}\right), 129.0,127.3,127.0$ (d, $\left.J_{C-F}=8.1 \mathrm{~Hz}\right), 126.4,125.2,123.8$, $88.8\left(\mathrm{~d}, J_{C-F}=185.3 \mathrm{~Hz}\right), 52.9 ;{ }^{19} \mathrm{~F} \mathrm{NMR}\left(376 \mathrm{MHz}, \mathrm{CDCl}_{3}\right): \delta-178.0(\mathrm{~d}, J=46.8 \mathrm{~Hz})$. This compound has been previously reported. ${ }^{\mathrm{S} 14}$<smiles>O=C(OCc1ccccc1)C(F)c1cccc2ccccc12</smiles>

12j

Benzyl 2-fluoro-2-(naphthalen-1-yl)acetate 12j. Yield: 56\% (165 mg, $560 \mu \mathrm{mol}$ ). Solvent system for flash column chromatography: petroleum ether/EtOAc: 99/1; Colorless oil; ${ }^{1} \mathrm{H}$ NMR (400 MHz, $\left.\mathrm{CDCl}_{3}\right): \delta$ 8.19-8.13 (m, 1H), 7.93-7.87 (m, 2H), 7.60-7.45 (m, 4H), 7.28-7.26 (m, 3H), 7.21-7.15 (m, 2H), 6.40 (d, J J-F $=47.1 \mathrm{~Hz}, 1 \mathrm{H}), 5.27$ (d, J = $12.3 \mathrm{~Hz}, 1 \mathrm{H}), 5.14(\mathrm{~d}, J=12.3 \mathrm{~Hz}, 1 \mathrm{H}$ ); ${ }^{13} \mathrm{C} \mathrm{NMR}\left(100 \mathrm{MHz}, \mathrm{CDCl}_{3}\right): \delta 168.8\left(\mathrm{~d}, J_{C-F}=27.8 \mathrm{~Hz}\right), 135.0,134.0,130.7(2 \mathrm{C}), 130.1\left(\mathrm{~d}, J_{C-F}=\right.$ $18.6 \mathrm{~Hz}), 128.9,128.6,128.5,128.2,127.1,127.0$ (d, $\left.J_{C-F}=7.9 \mathrm{~Hz}\right), 126.3,125.2,123.8,88.7$ (d, $\left.J_{C-F}=185.9 \mathrm{~Hz}\right), 67.4 ;{ }^{19} \mathrm{~F} \mathrm{NMR}\left(376 \mathrm{MHz}, \mathrm{CDCl}_{3}\right): \delta-177.9$ (d, $\left.J=46.9 \mathrm{~Hz}\right) ; \mathrm{IR}(\mathrm{ATR}): v_{\max } 1736$, 1455, 1264, 1213, 1167, 1146, 1047, 970, 781, 739, $697 \mathrm{~cm}^{-1}$; ESI-HRMS m/z calcd for $\mathrm{C}_{19} \mathrm{H}_{19} \mathrm{FNO}_{2}\left[\mathrm{M}+\mathrm{NH}_{4}\right]^{+}$312.1394, found 312.1387.

S14 Paull, D. T.; Scerba, M. T.; Alden-Danforth, E.; Widger L. R.; Lectka, T. J. Am. Chem. Soc. 2008, 130, 17260. 


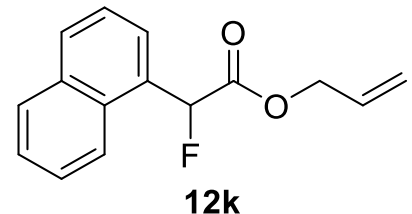

Allyl 2-fluoro-2-(naphthalen-1-yl)acetate 12k. Yield: Isolation of $101 \mathrm{mg}$ of the fluorinated product $(40 \%)$ contaminated with the corresponding protonated byproduct (1\%). Solvent system for flash column chromatography: petroleum ether/EtOAc: 99/1; Colorless oil; ${ }^{1} \mathrm{H}$ NMR $\left(400 \mathrm{MHz}, \mathrm{CDCl}_{3}\right): \delta 8.20(\mathrm{~d}, J=8.3 \mathrm{~Hz}, 1 \mathrm{H}), 7.94-7.88(\mathrm{~m}, 2 \mathrm{H}), 7.64-7.47(\mathrm{~m}, 4 \mathrm{H}), 6.40\left(\mathrm{~d}, J_{H-F}\right.$ $=47.0 \mathrm{~Hz}, 1 \mathrm{H}$ ), 5.82 (M of ABMXY syst., $J=17.2,10.5$ and $5.7 \mathrm{~Hz}, 1 \mathrm{H}$ ), 5.19 (X of ABMXY syst., $J=8.0$ and $1.3 \mathrm{~Hz}, 1 \mathrm{H}), 5,16-5,14(\mathrm{~m}, 1 \mathrm{H}), 4.71(\mathrm{~A}$ of $\mathrm{ABMXY}$ syst., $J=13.2,5.7$ and $1.4 \mathrm{~Hz}, 1 \mathrm{H})$, 4.67 (B of ABMXY syst., $J=13.3,5.8$ and $1.5 \mathrm{~Hz}, 1 \mathrm{H}) ;{ }^{13} \mathrm{C}$ NMR $\left(100 \mathrm{MHz}, \mathrm{CDCl}_{3}\right): \delta 168.7$ (d, $J_{C-}$ $F=27.8 \mathrm{~Hz}), 134.0,131.2,130.8,130.7,130.2\left(\mathrm{~d}, J_{C-F}=19.0 \mathrm{~Hz}\right), 128.9,127.2,127.0\left(\mathrm{~d}, J_{C-F}=\right.$ 8.0 Hz), 126.4, 125.2, 123.9, 119.1, $88.7\left(\mathrm{~d}, J_{\mathrm{C}-\mathrm{F}}=185.7 \mathrm{~Hz}\right), 66.3 ;{ }^{19} \mathrm{~F} \mathrm{NMR}\left(376 \mathrm{MHz}, \mathrm{CDCl}_{3}\right)$ : $\delta-177.9\left(\mathrm{~d}, J=46.7 \mathrm{~Hz}\right.$ ); IR (ATR): $v_{\max } 2922,2852,1758,1513,1456,1199,788,774 \mathrm{~cm}^{-1}$; ESIHRMS m/z calcd for $\mathrm{C}_{15} \mathrm{H}_{17} \mathrm{FNO}_{2}\left[\mathrm{M}+\mathrm{NH}_{4}\right]^{+} 262.1238$, found 262.1240 .

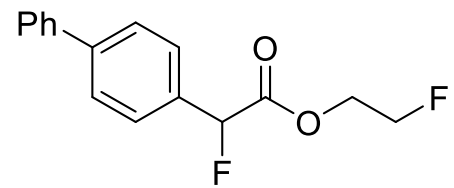

121

Benzyl 2-fluoro-2-(naphthalen-1-yl)acetate 12I. Yield: Isolation of $100 \mathrm{mg}$ of the fluorinated product (34\%) contaminated with the corresponding protonated byproduct $(2 \%)$. Solvent system for flash column chromatography: petroleum ether/EtOAc: 95/5 to 90/10; Yellow oil; ${ }^{1} \mathrm{H} \mathrm{NMR}\left(400 \mathrm{MHz}, \mathrm{CDCl}_{3}\right): \delta 7.64(\mathrm{~d}, J=7.7 \mathrm{~Hz}, 2 \mathrm{H}), 7.61-7.54(\mathrm{~m}, 4 \mathrm{H}), 7.46(\mathrm{t}, J=7.4 \mathrm{~Hz}, 2 \mathrm{H})$, 7.43-7.32 (m, 1H), $5.89\left(\mathrm{~d}, J_{H-F}=47.5 \mathrm{~Hz}, 1 \mathrm{H}\right), 4.71-4.63(\mathrm{~m}, 1 \mathrm{H}), 4.57-4.53(\mathrm{~m}, 1 \mathrm{H}), 4.53-4.32$ (m, 2H); ${ }^{13} \mathrm{C} \mathrm{NMR} \mathrm{(100} \mathrm{MHz,} \mathrm{CDCl} 3$ ): $\delta 168.5$ (d, J = 28.1 Hz), 142.9 (d, J = $2.3 \mathrm{~Hz}$ ), 140.4, 132.9 ( $d, J=20.5 \mathrm{~Hz}$ ), 129.0, 127.9, 127.8, 127.3, 127.3, 89.2 (d, $J=186.0 \mathrm{~Hz}$ ), 80.9 (d, $J=171.7 \mathrm{~Hz})$, $64.6(\mathrm{~d}, J=20.5 \mathrm{~Hz}) ;{ }^{19} \mathrm{~F} \mathrm{NMR}\left(376 \mathrm{MHz}, \mathrm{CDCl}_{3}\right): \delta-179.7$ (d, $J=47.5 \mathrm{~Hz}$ ), -224.9 (tt, $J=47.2$ and $28.1 \mathrm{~Hz}$ ); IR (ATR): $v_{\max } 2957,2923,2852,1763,1487,1262,1213,1187,1065,876,840$, 760, 736, $698 \mathrm{~cm}^{-1}$; ESI-HRMS m/z calcd for $\mathrm{C}_{16} \mathrm{H}_{18} \mathrm{~F}_{2} \mathrm{NO}_{2}\left[\mathrm{M}+\mathrm{NH}_{4}\right]^{+}$294.1300, found 294.1295. 


\section{Experimental Procedures and Characterization Data: $\alpha$-Fluoroamides}

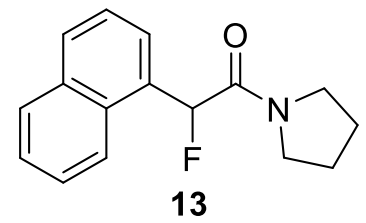

2-Fluoro-2-(naphthalen-1-yl)-1-(pyrrolidin-1-yl)ethan-1-one 13. To a solution of amide (1.0 mmol) and 2-bromopyridine $(210 \mu \mathrm{L}, 2.2 \mathrm{mmol})$ in dry dichloromethane $(3 \mathrm{~mL})$ at $0{ }^{\circ} \mathrm{C}$ was added dropwise triflic anhydride $(250 \mu \mathrm{L}, 1.5 \mathrm{mmol})$. The resulting brown solution was stirred for 5 minutes at $0{ }^{\circ} \mathrm{C}$ then $\mathrm{N}$-fluorobenzenesulfonimide (631 mg, $2.0 \mathrm{mmol}$ ) and benzoic acid (244 mg, $2.0 \mathrm{mmol}$ ) were successively added in one portion. The reaction mixture was stirred at $40{ }^{\circ} \mathrm{C}$ overnight, cooled down to rt and stopped by addition of a $1 \mathrm{M}$ solution of $\mathrm{NaOH}(5$ $\mathrm{mL}$ ). The biphasic mixture was vigorously stirred for 1 hour then diluted with dichloromethane. The organic phase was washed with a $1 \mathrm{M}$ solution of $\mathrm{NaOH}(10 \mathrm{~mL})$, with a $1 \mathrm{M}$ solution of $\mathrm{HCl}(10 \mathrm{~mL})$, dried over $\mathrm{MgSO}_{4}$, filtered and concentrated under reduced pressure. The crude residue was finally purified by flash column chromatography over silica gel (petroleum ether/EtOAc: 80/20 to 70/30) to yield 2-fluoro-2-(naphthalen-1-yl)-1-(pyrrolidin-1yl)ethan-1-one 13 (130 mg, $506 \mu \mathrm{mol}, 51 \%)$. Yellow oil; ${ }^{1} \mathrm{H}$ NMR (400 MHz, $\mathrm{CDCl}_{3}$ ): $\delta 8.30$ (d, J $=8.4 \mathrm{~Hz}, 1 \mathrm{H}), 7.93-7.88(\mathrm{~m}, 2 \mathrm{H}), 7.63-7.52(\mathrm{~m}, 3 \mathrm{H}), 7.50-7.45(\mathrm{~m}, 1 \mathrm{H}), 6.56(\mathrm{~d}, J=48.4 \mathrm{~Hz}, 1 \mathrm{H})$, 3.69-3.53 (m, 2H), 3.49-3.37 (m, 1H), 3.12-3.05 (m, 1H), 1.90-1.74 (m, 4H); ${ }^{13} \mathrm{C} \mathrm{NMR}(100 \mathrm{MHz}$, $\left.\mathrm{CDCl}_{3}\right): \delta 166.6\left(\mathrm{~d}, J_{C-F}=23.8 \mathrm{~Hz}\right), 134.1,131.6,130.8\left(\mathrm{~d}, J_{C-F}=3.3 \mathrm{~Hz}\right), 129.9\left(\mathrm{~d}, J_{C-F}=18.5 \mathrm{~Hz}\right)$, $128.9,127.3,126.6\left(\mathrm{~d}, J_{C-F}=6.7 \mathrm{~Hz}\right), 126.4,125.2\left(\mathrm{~d}, J_{C-F}=1.8 \mathrm{~Hz}\right), 123.8,89.1\left(\mathrm{~d}, J_{C-F}=181.9\right.$ $\mathrm{Hz}), 46.8,46.2\left(\mathrm{~d}, J_{C-F}=3.1 \mathrm{~Hz}\right), 26.4,23.7 ;{ }^{19} \mathrm{~F} \mathrm{NMR}\left(376 \mathrm{MHz}, \mathrm{CDCl}_{3}\right): \delta-172.2$ (d, $\left.J=48.4 \mathrm{~Hz}\right)$; IR (ATR): $v_{\max } 2972,2879,1654,1548,1510,1450,1340,1190,1168,1060,1035,804,784$, $698 \mathrm{~cm}^{-1}$;ESI-HRMS m/z calcd for $\mathrm{C}_{16} \mathrm{H}_{17} \mathrm{FNO}[\mathrm{M}+\mathrm{H}]^{+}$258.1289, found 258.1293. 


\section{Experimental Procedures and Characterization Data: $\alpha$-Fluorocarboxylic Acids}<smiles>O=C(O)C(F)c1ccc(-c2ccccc2)cc1</smiles>

2-Fluoro-2-([1,1'-biphenyl]-4-yl) fluoroacetic acid 14. To a solution of ethyl 2-fluoro-2-([1,1'biphenyl]-4-yl)acetate $12 \mathrm{~b}$ (118 mg, $460 \mu \mathrm{mol})$ in ethanol $(2 \mathrm{~mL})$ was added a $18 \mathrm{M}$ aqueous solution of sodium hydroxide $(270 \mu \mathrm{L})$. The resulting white mixture was stirred overnight at 80 ${ }^{\circ} \mathrm{C}$ and stopped by addition of $1 \mathrm{M}$ aqueous solution of $\mathrm{HCl}(5 \mathrm{~mL})$. The aqueous layer was extracted twice with ethyl acetate $(2 \times 15 \mathrm{~mL})$ and the combined organic layers were dried over $\mathrm{MgSO}_{4}$, filtered and concentrated under reduced pressure. The crude residue was finally purified by flash column chromatography over silica gel (petroleum ether/EtOAc: 90/10) to yield $61 \mathrm{mg}$ of a mixture of the fluorinated product (58\%) contaminated with the corresponding protonated byproduct (6\%). ${ }^{1} \mathrm{H}$ NMR $\left(400 \mathrm{MHz}, \mathrm{CD}_{3} \mathrm{OD}\right){ }^{\mathrm{S} 15}: \delta 7.67(\mathrm{~d}, J=7.8 \mathrm{~Hz}, 2 \mathrm{H}), 7.65-7.61$ (m, $2 \mathrm{H}), 7.55(\mathrm{~d}, J=8.1 \mathrm{~Hz}, 2 \mathrm{H}), 7.47-7.41(\mathrm{~m}, 2 \mathrm{H}), 7.37-7.34(\mathrm{~m}, 1 \mathrm{H}), 5.89\left(\mathrm{~d}, J_{H-F}=48.0 \mathrm{~Hz}\right.$, $1 \mathrm{H}) ;{ }^{13} \mathrm{C} N M R\left(100 \mathrm{MHz}, \mathrm{CD}_{3} \mathrm{OD}\right): \delta 172.1\left(\mathrm{~d}, J_{C-F}=27.7 \mathrm{~Hz}\right), 143.6\left(\mathrm{~d}, J_{C-F}=2.3 \mathrm{~Hz}\right), 141.5,135.3$ $\left(\mathrm{d}, J_{C-F}=20.4 \mathrm{~Hz}\right), 129.9,128.7,128.4\left(\mathrm{~d}, J_{C-F}=5.7 \mathrm{~Hz}\right), 128.3,128.0,90.3\left(\mathrm{~d}, J_{C-F}=182.0 \mathrm{~Hz}\right)$; ${ }^{19} \mathrm{~F}$ NMR $\left(376 \mathrm{MHz}, \mathrm{CDCl}_{3}\right): \delta-177.0(\mathrm{~d}, J=48.2 \mathrm{~Hz})$. This compound has been previously reported. $\mathrm{S16}$

\footnotetext{
S15 The labile proton of the acid could not be observed.

S16 Mizuta, S.; Stenhagen, I. S. R.; O’Duill, M.; Wolstenhulme, J.; Kirjavainen, A. K.; Forsback, S. J.; Tredwell, M.; Sandford, G.; Moore, P. R.; Huiban, M.; Luthra, S. K.; Passchier, J.; Solin, O.; Gouverneur, V. Org. Lett. 2013, 15, 2648.
} 
<smiles>O=C(O)C(F)c1ccccc1Nc1c(Cl)cccc1Cl</smiles>

15

2-(2-((2,6-Dichlorophenyl)amino)phenyl)-2-fluoroacetic acid 15. A pressure tube was charged with arylamine (97 mg, $600 \mu \mathrm{mol}), \mathrm{PdCl}_{2}(0.4 \mathrm{mg}, 2.5 \mu \mathrm{mol})$ and DPEPhos ( $\left.2 \mathrm{mg}, 3.8 \mu \mathrm{mol}\right)$. The tube was fitted with a rubber septum, evacuated under high vacuum and backfilled with argon three times. Anhydrous toluene (0.5-1.0 M) was next added, the rubber septum was replaced by a Teflon-coated screw cap and the mixture was stirred at $80{ }^{\circ} \mathrm{C}$ for $30 \mathrm{~min}$. The tube was then cooled to $\mathrm{rt}$, and aryl iodide $(185 \mathrm{mg}, 500 \mu \mathrm{mol})$ followed by sodium tert-butoxide $(67 \mathrm{mg}, 700$ $\mu \mathrm{mol}$ ) was added. An additional portion of toluene was then added to wash any base adhering to the side of the tube. A flow of argon was passed for 5 min to ensure a protective atmosphere. The tube was sealed with a Teflon-coated screw cap and heated at $80^{\circ} \mathrm{C}$ for $16 \mathrm{~h}$. The reaction mixture was then cooled down to $\mathrm{rt}$, taken up with diethylether $(10 \mathrm{~mL})$ and washer with brine $(10 \mathrm{~mL})$. The organic layer was dried over $\mathrm{MgSO}_{4}$, filtered and concentrated under reduced pressure. To this crude residue in ethanol $(3 \mathrm{~mL})$ was added a $18 \mathrm{M}$ aqueous solution of sodium hydroxide $(500 \mu \mathrm{L})$. The resulting white mixture was stirred for $5 \mathrm{~h}$ at $80^{\circ} \mathrm{C}$ and stopped by addition of $1 \mathrm{M}$ aqueous solution of $\mathrm{HCl}(10 \mathrm{~mL})$. The aqueous layer was extracted twice with ethyl acetate $(2 \times 20 \mathrm{~mL})$ and the combined organic layers were dried over $\mathrm{MgSO}_{4}$, filtered and concentrated under reduced pressure. The crude residue was finally purified by flash column chromatography over silica gel (petroleum ether/EtOAc: $90 / 10$ to $80 / 20)$ to yield 2-(2-((2,6dichlorophenyl)amino)phenyl)-2-fluoroacetic acid 15 (55 mg, 35\%). Off-white solid; Mp: 140 ${ }^{\circ} \mathrm{C} ;{ }^{1} \mathrm{H}$ NMR (400 MHz, $\left.\mathrm{CDCl}_{3}\right)^{\mathrm{S} 15}: \delta 8.04$ (bs, $1 \mathrm{H}$ ), 7.93 (dt, $J=8.0$ and $1.2 \mathrm{~Hz}, 1 \mathrm{H}$ ), $7.63(\mathrm{dt}, J=$ 7.8 and $1.5 \mathrm{~Hz}, 1 \mathrm{H}), 7.45-7.39(\mathrm{~m}, 3 \mathrm{H}), 7.26-7.21(\mathrm{~m}, 1 \mathrm{H}), 7.12(\mathrm{ddt}, J=8.0,7.4$ and $1.8 \mathrm{~Hz}, 1 \mathrm{H})$, $6.35\left(\mathrm{~d}, J_{C-F}=47.5 \mathrm{~Hz}, 1 \mathrm{H}\right) ;{ }^{13} \mathrm{C} \mathrm{NMR}\left(100 \mathrm{MHz}, \mathrm{CDCl}_{3}\right): \delta 166.2\left(\mathrm{~d}, J_{C-F}=22.8 \mathrm{~Hz}\right), 140.2,137.4$ $\left(d, J_{C-F}=18.5 \mathrm{~Hz}\right), 133.9,131.7\left(\mathrm{~d}, J_{C-F}=3.3 \mathrm{~Hz}\right), 130.7,129.3,129.0,128.9,128.7,100.4\left(\mathrm{~d}, J_{C-}\right.$ $F=4.8 \mathrm{~Hz}$ ), $95.2\left(\mathrm{~d}, J_{C-F}=188.8 \mathrm{~Hz}\right) ;{ }^{19} \mathrm{~F} \mathrm{NMR}\left(376 \mathrm{MHz}, \mathrm{CDCl}_{3}\right): \delta-170.8(\mathrm{~d}, J=47.3 \mathrm{~Hz}) . \mathrm{IR}$ (ATR): $v_{\max } 3393,3258,1697,1568,1500,1452,1436,1277,1197,1103,1012,783,751 \mathrm{~cm}^{-1}$; ESI-HRMS m/z calcd for $\mathrm{C}_{14} \mathrm{H}_{10} \mathrm{FKNO}_{2}[\mathrm{M}-\mathrm{K}]^{+} 351.9704$, found 351.9691 . 


\section{GC Spectra}

GC analyses were recorded on a Thermo Finningan Trace GC apparatus with a HIRALSIL-DEX $\mathrm{CB}(25 \mathrm{~m}, 0.25 \mathrm{~mm}, 25 \mu \mathrm{m})$ column. Chiral GC: $\mathrm{T}=130^{\circ} \mathrm{C}(80 \mathrm{~min}) .^{\mathrm{S} 17}$

\section{Racemic ethyl 2-fluoro-2-(naphthalen-1-yl)acetate from 9c}<smiles>O=C(Cc1cccc2ccccc12)N1CCCC1</smiles>

9c

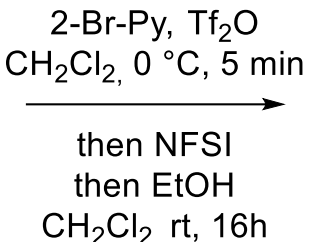

$\mathrm{CH}_{2} \mathrm{Cl}_{2}$, rt, $16 \mathrm{~h}$<smiles>CCOC(=O)C(F)c1cccc2ccccc12</smiles>

12c

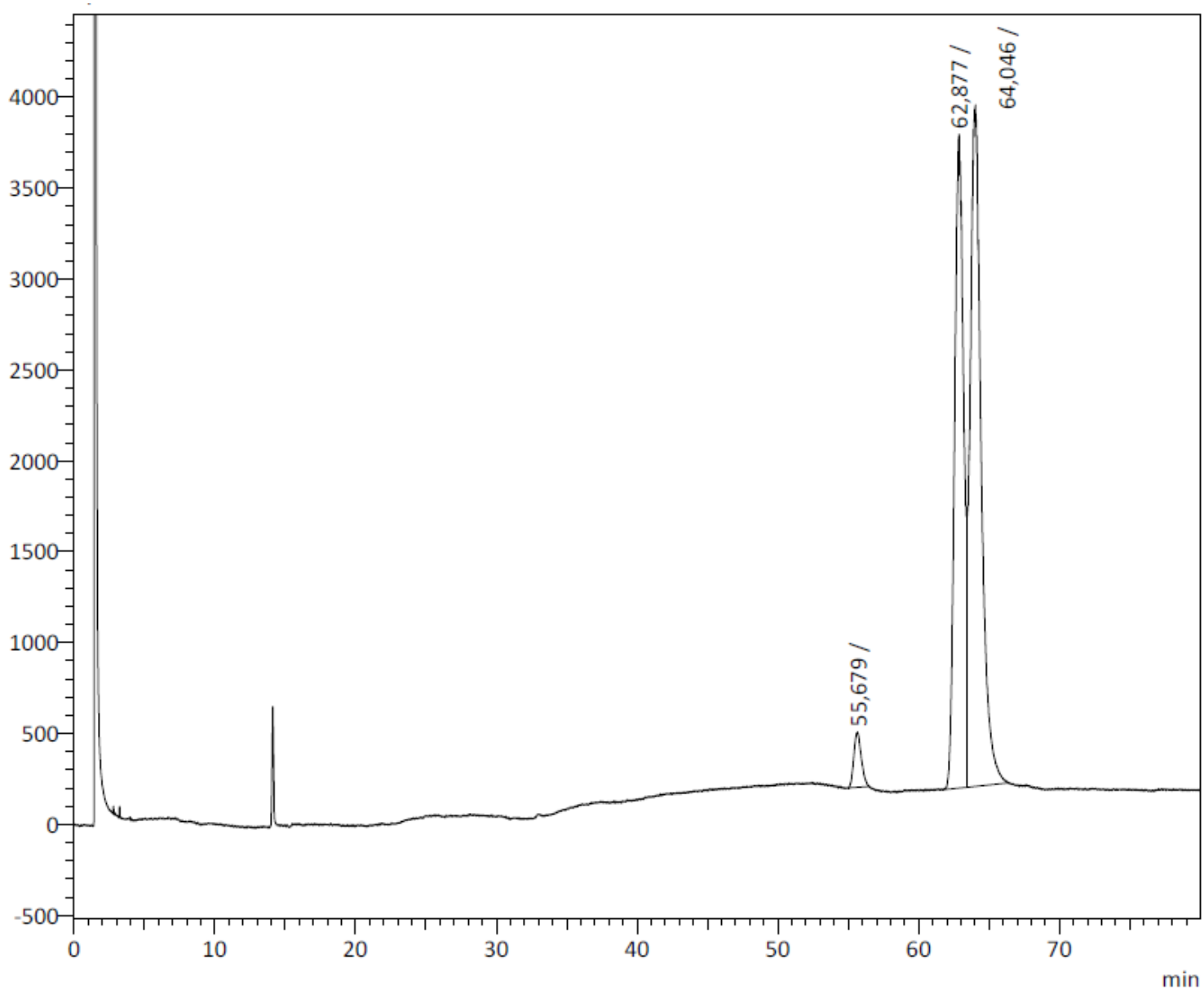

Peak Table - Channel 1

\begin{tabular}{|r|r|r|r|r|l|}
\hline Peak\# & Ret. Time & \multicolumn{1}{|c|}{ Area } & Height & Area\% & Name \\
\hline 1 & 55,679 & 11121 & 304 & 2,9486 & \\
\hline 2 & 62,877 & 158147 & 3596 & 41,9311 & \\
\hline 3 & 64,046 & 207891 & 3744 & 55,1203 & \\
\hline Total & & 377159 & 7644 & 100,0000 & \\
\hline
\end{tabular}

s17 The signal at 55.679min was identified as ethyl 2-(naphthalen-1-yl)acetate. 
Ethyl 2-fluoro-2-(naphthalen-1-yl)acetate from 9b*<smiles>C[C@H](C(O)c1ccccc1)N(C)C(=O)Cc1cccc2ccccc12</smiles>

$9 b^{*}$

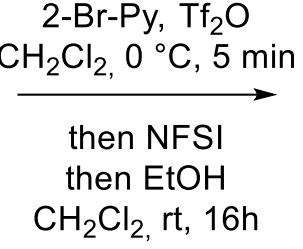

$\mathrm{CH}_{2} \mathrm{Cl}_{2}$, rt, $16 \mathrm{~h}$<smiles>CCOC(=O)C(F)c1cccc2ccccc12</smiles>

$12 c^{*}$

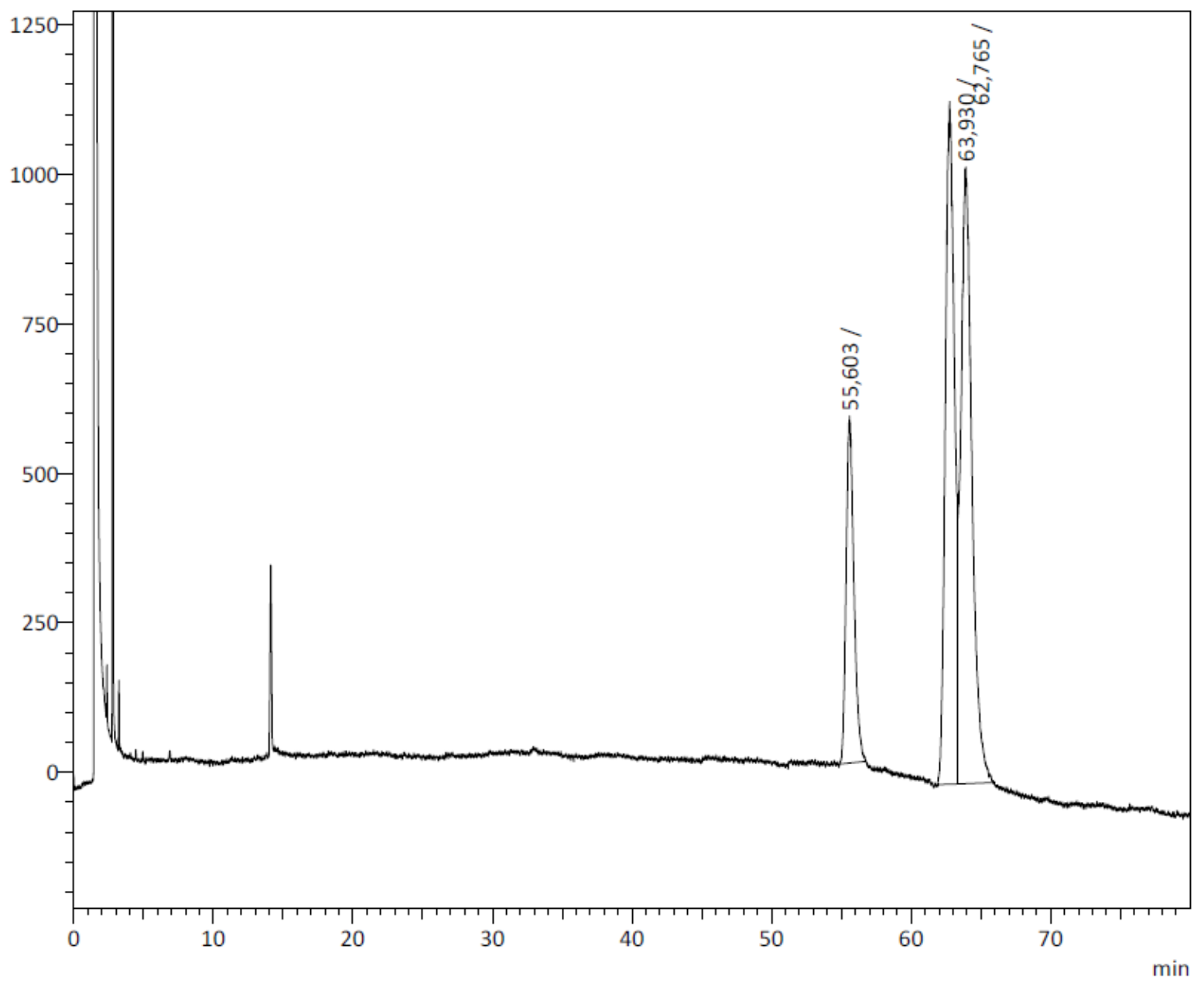

Peak Table - Channel 1

\begin{tabular}{|r|r|r|r|r|l|}
\hline \multicolumn{1}{|c|}{ Peak\# } & Ret. Time & \multicolumn{1}{c|}{ Area } & Height & \multicolumn{1}{c|}{ Area\% } & Name \\
\hline 1 & 55,603 & 22313 & 579 & 17,0204 & \\
\hline 2 & 62,765 & 50817 & 1141 & 38,7627 & \\
\hline 3 & 63,930 & 57967 & 1030 & 44,2170 & \\
\hline Total & & 131097 & 2750 & 100,0000 & \\
\hline
\end{tabular}


Ethyl 2-fluoro-2-(naphthalen-1-yl)acetate from 9d*<smiles>COC[C@@H]1CCCN1C(=O)Cc1cccc2ccccc12</smiles>

$9 d^{*}$
2-Br-Py, $\mathrm{Tf}_{2} \mathrm{O}$ $\mathrm{CH}_{2} \mathrm{Cl}_{2}, 0^{\circ} \mathrm{C}, 5 \mathrm{~min}$

then NFSI then $\mathrm{EtOH}$ $\mathrm{CH}_{2} \mathrm{Cl}_{2}, \mathrm{rt}, 16 \mathrm{~h}$<smiles>CCOC(=O)[C@H](F)c1cccc2ccccc12</smiles>

$12 c^{*}$

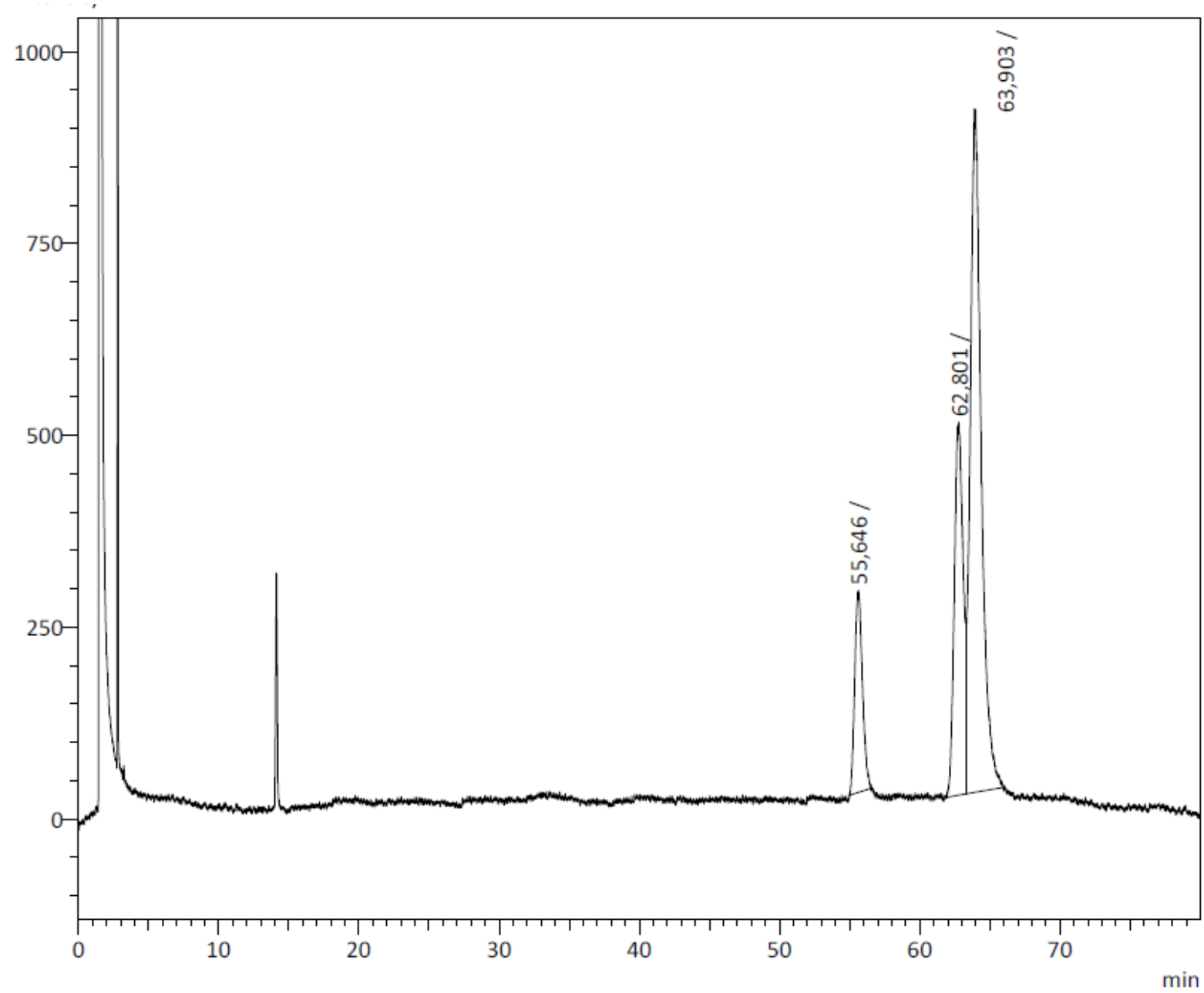

Peak Table - Channel 1

\begin{tabular}{|r|r|r|r|r|l|}
\hline Peak\# & Ret. Time & \multicolumn{1}{c|}{ Area } & Height & \multicolumn{1}{c|}{ Area\% } & Name \\
\hline 1 & 55,646 & 9993 & 263 & 12,5239 & \\
\hline 2 & 62,801 & 21362 & 486 & 26,7708 & \\
\hline 3 & 63,903 & 48440 & 891 & 60,7053 & \\
\hline Total & & 79795 & 1640 & 100,0000 & \\
\hline
\end{tabular}


Ethyl 2-fluoro-2-(naphthalen-1-yl)acetate from 9e*<smiles>CC1CC[C@@H](C)N1C(=O)Cc1cccc2ccccc12</smiles>

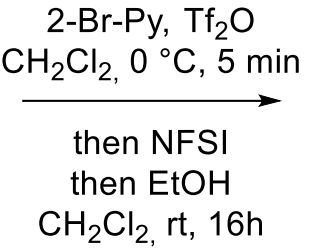<smiles>CCOC(=O)[C@H](F)c1cccc2ccccc12</smiles>

$12 c^{*}$

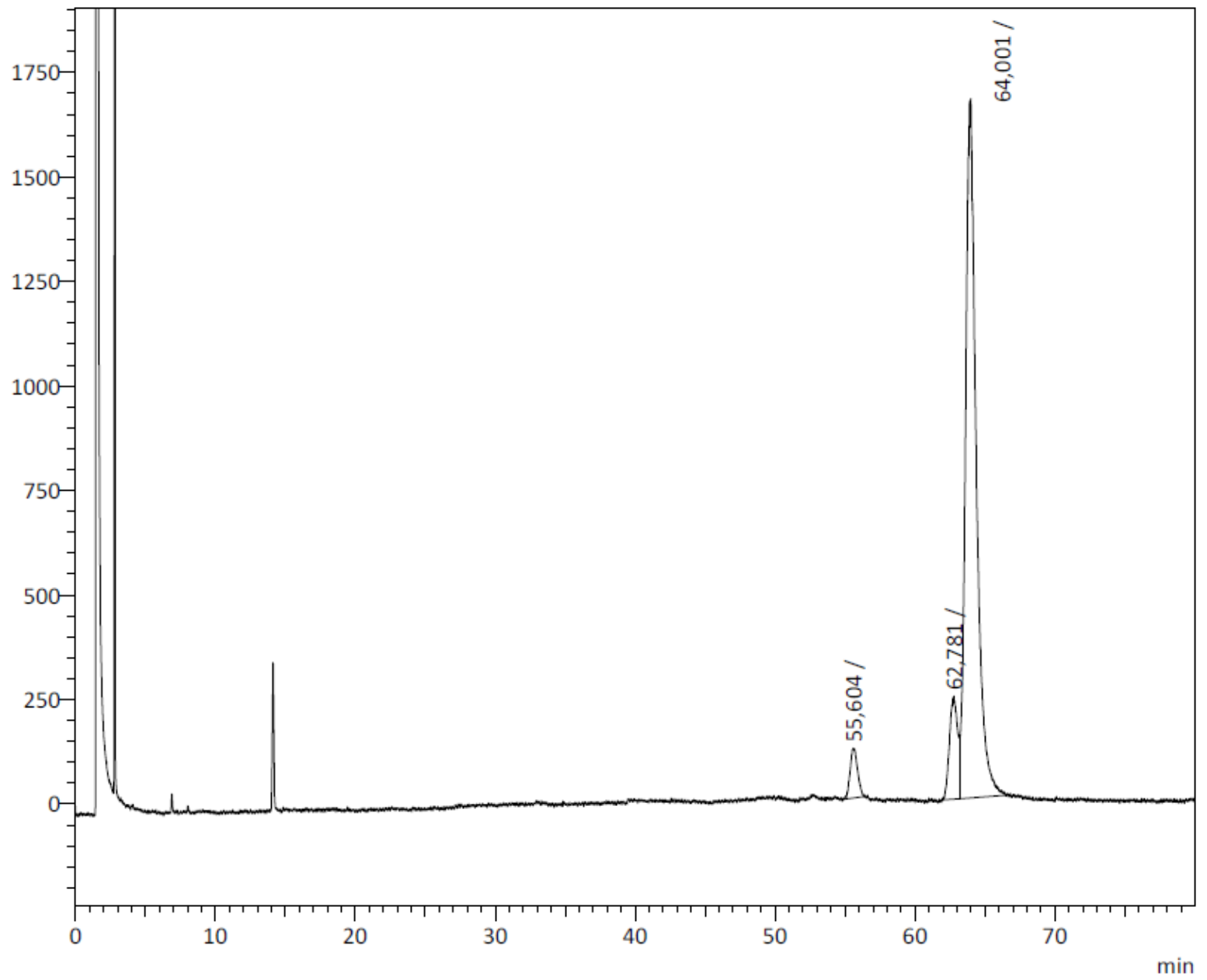

Peak Table - Channel 1

\begin{tabular}{|r|r|r|r|r|r|}
\hline Peak\# & Ret. Time & \multicolumn{1}{|c|}{ Area } & Height & \multicolumn{1}{c|}{ Area\% } & Name \\
\hline 1 & 55,604 & 4422 & 119 & 4,2912 & \\
\hline 2 & 62,781 & 9804 & 247 & 9,5136 & \\
\hline 3 & 64,001 & 88831 & 1672 & 86,1952 & \\
\hline Total & & 103057 & 2038 & 100,0000 & \\
\hline
\end{tabular}


Ethyl 2-fluoro-2-(naphthalen-1-yl)acetate from $9 f * 518$<smiles>O=C(Cc1cccc2ccccc12)N1[C@H](c2ccccc2)CC[C@H]1c1ccccc1</smiles>

9f*
2-Br-Py, $\mathrm{Tf}_{2} \mathrm{O}$ $\mathrm{CH}_{2} \mathrm{Cl}_{2}, 0^{\circ} \mathrm{C}, 5 \mathrm{~min}$

then NFSI then $\mathrm{EtOH}$ $\mathrm{CH}_{2} \mathrm{Cl}_{2}, \mathrm{rt}, 16 \mathrm{~h}$<smiles>CCOC(=O)[C@H](F)c1cccc2ccccc12</smiles>

$12 c^{*}$

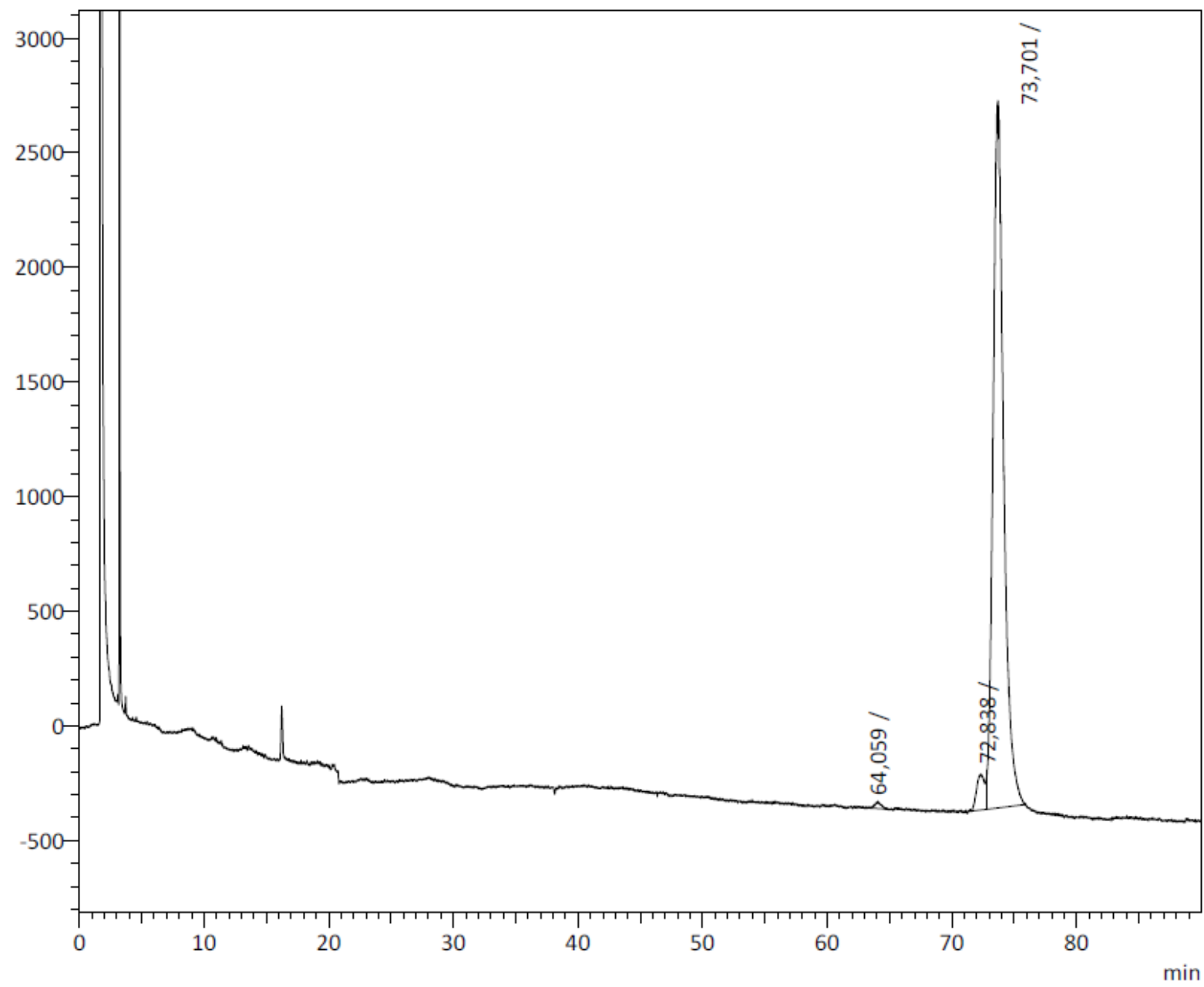

\begin{tabular}{|r|r|r|r|r|l|}
\multicolumn{7}{|c|}{ Peak Table - Channel 1 } \\
\hline Peak\# & Ret. Time & \multicolumn{1}{|c|}{ Area } & Height & \multicolumn{1}{|c|}{ Area $\%$} & Name \\
\hline 1 & 64,059 & 1034 & 31 & 0,5449 & \\
\hline 2 & 72,838 & 7722 & 161 & 4,0711 & \\
\hline 3 & 73,701 & 180929 & 3083 & 95,3840 & \\
\hline Total & & 189685 & 3275 & 100,0000 & \\
\hline
\end{tabular}

S18 A difference in absolute retention time was observed for the measurement. It was confirmed with the racemic product (not shown). However, relative retention time were unchanged. 


\section{Determination of the Absolute Stereochemistry}

The chiral induction observed, largely in favor of the $R$ enantiomer, can be rationalized based on the structure of the intermediate enamine resulting from the trapping of the transient keteniminium ion by the nucleophile. Initial kinetic approach of the nucleophile to the keteniminium ion provides a highly sterically hindered $E$ enamine which is in equilibrium with the less hindered $Z$ isomer. The approach of the electrophilic fluorination agent by the favored $R e$ face of the $\mathrm{N}, \mathrm{O}$-ketene acetal leads to the preferable formation of the $(R)$-product.

Absolute stereochemistry of the chiral center was determined by correlation with data from literature. ${ }^{\mathrm{S} 19}$
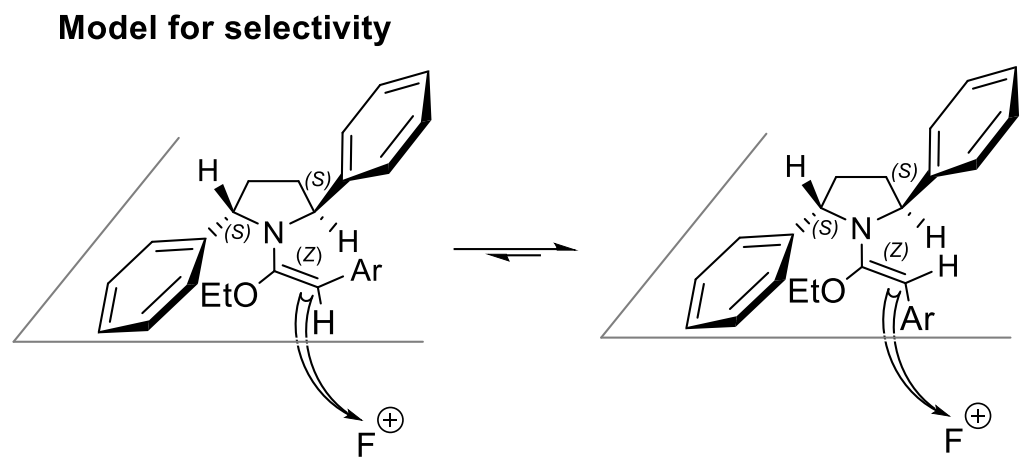

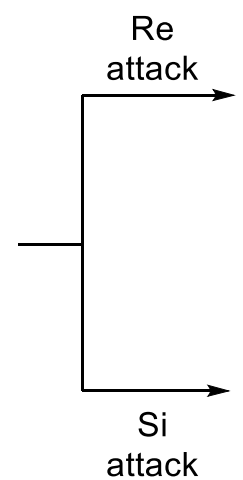

attack<smiles>CCOC(=O)[C@H](F)c1cccc2ccccc12</smiles>

Favored<smiles>CCOC(=O)[C](F)c1cccc2ccccc12</smiles>

\section{Disfavored}

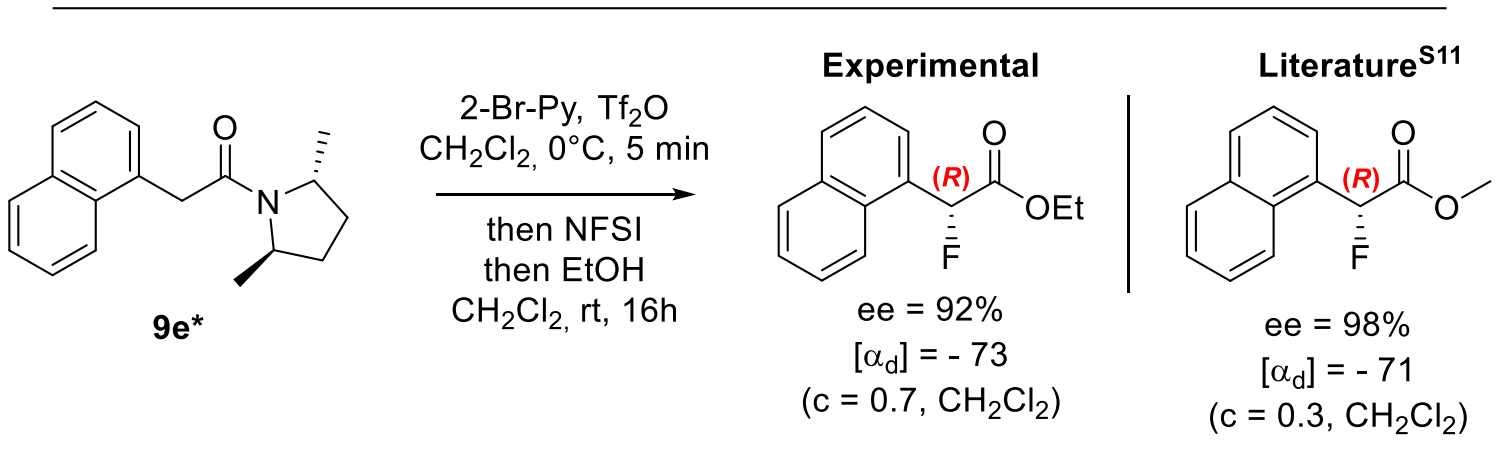

S19 Paull, D. H.; Scerba, M. T.; Alden-Danforth, E.; Widger, L. R.; Lectka, T. J. Am. Chem. Soc. 2008, $130,17260$. 


\section{${ }^{1} \mathrm{H},{ }^{13} \mathrm{C}$ and ${ }^{19} \mathrm{~F}$ NMR Spectra}




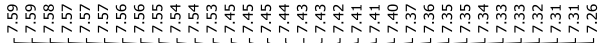<smiles>O=C(Cc1ccc(-c2ccccc2)cc1)N1CCCC1</smiles>

$\left(\mathrm{CDCl}_{3}, 400 \mathrm{MHz}\right)$

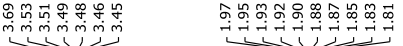
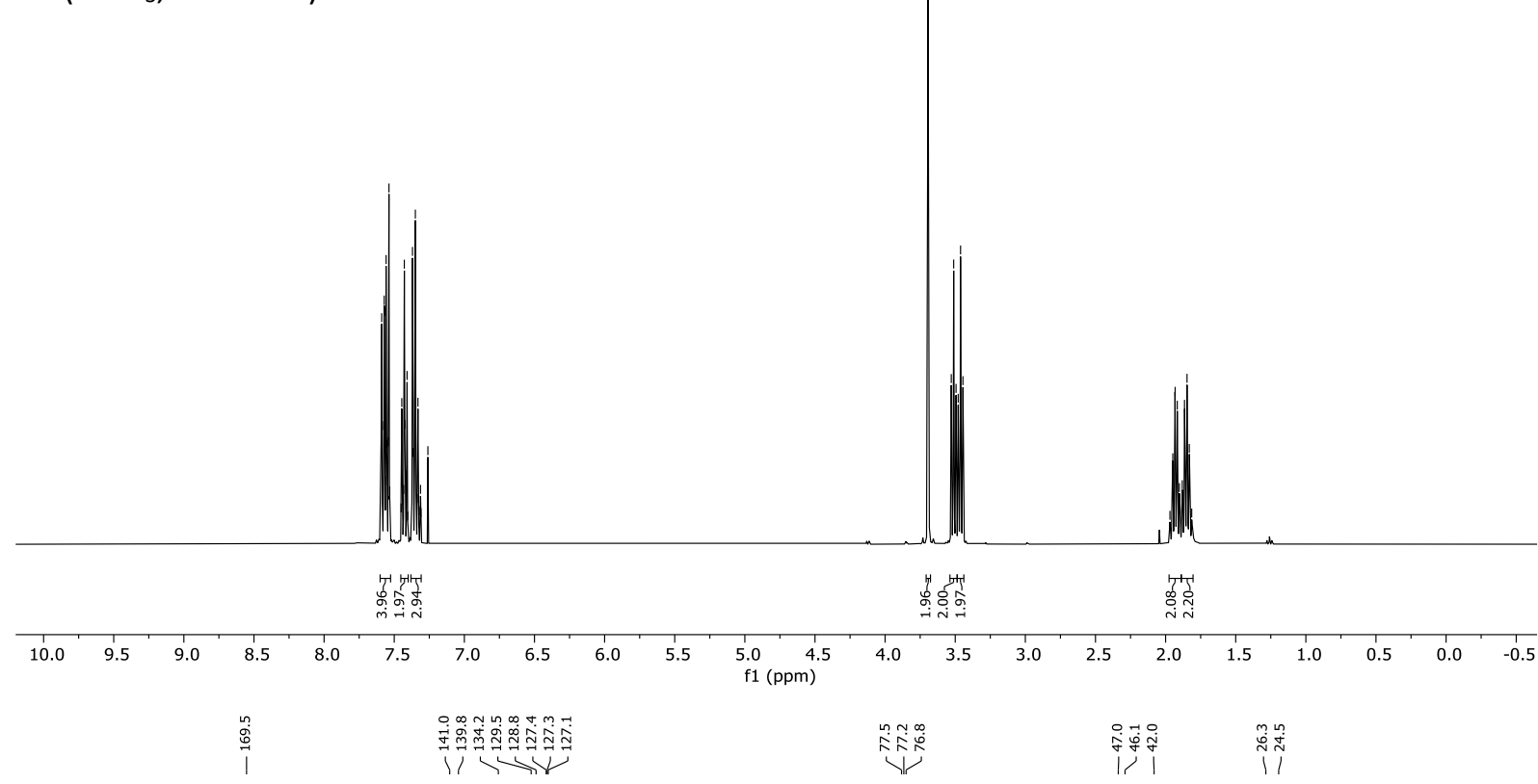

$\left(\mathrm{CDCl}_{3}, 100 \mathrm{MHz}\right)$

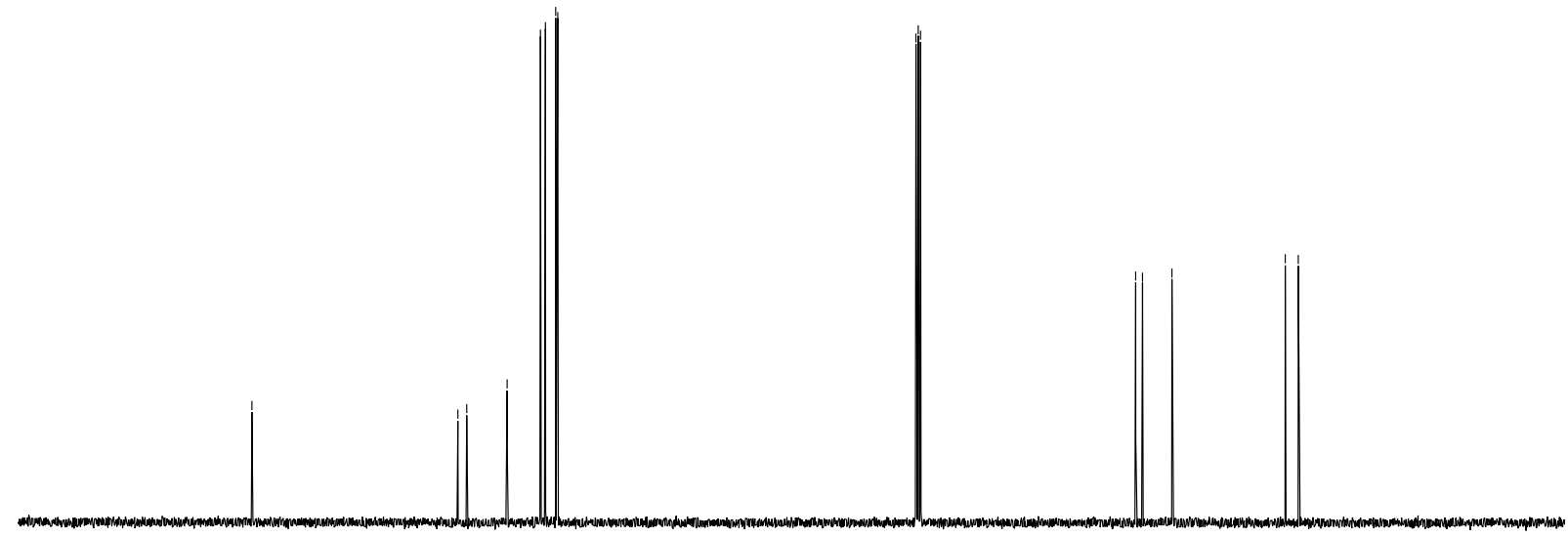

$\begin{array}{llllllllllllllllllllll} & 190 & 180 & 170 & 160 & 150 & 140 & 130 & 120 & 110 & 100 & 90 & 80 & 70 & 60 & 50 & 40 & 30 & 20 & 10 & 0 & -10\end{array}$ 


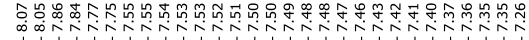<smiles>O=C(Cc1cccc2ccccc12)N1CCCC1</smiles>

$\left(\mathrm{CDCl}_{3}, 400 \mathrm{MHz}\right)$

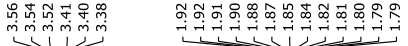

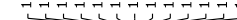

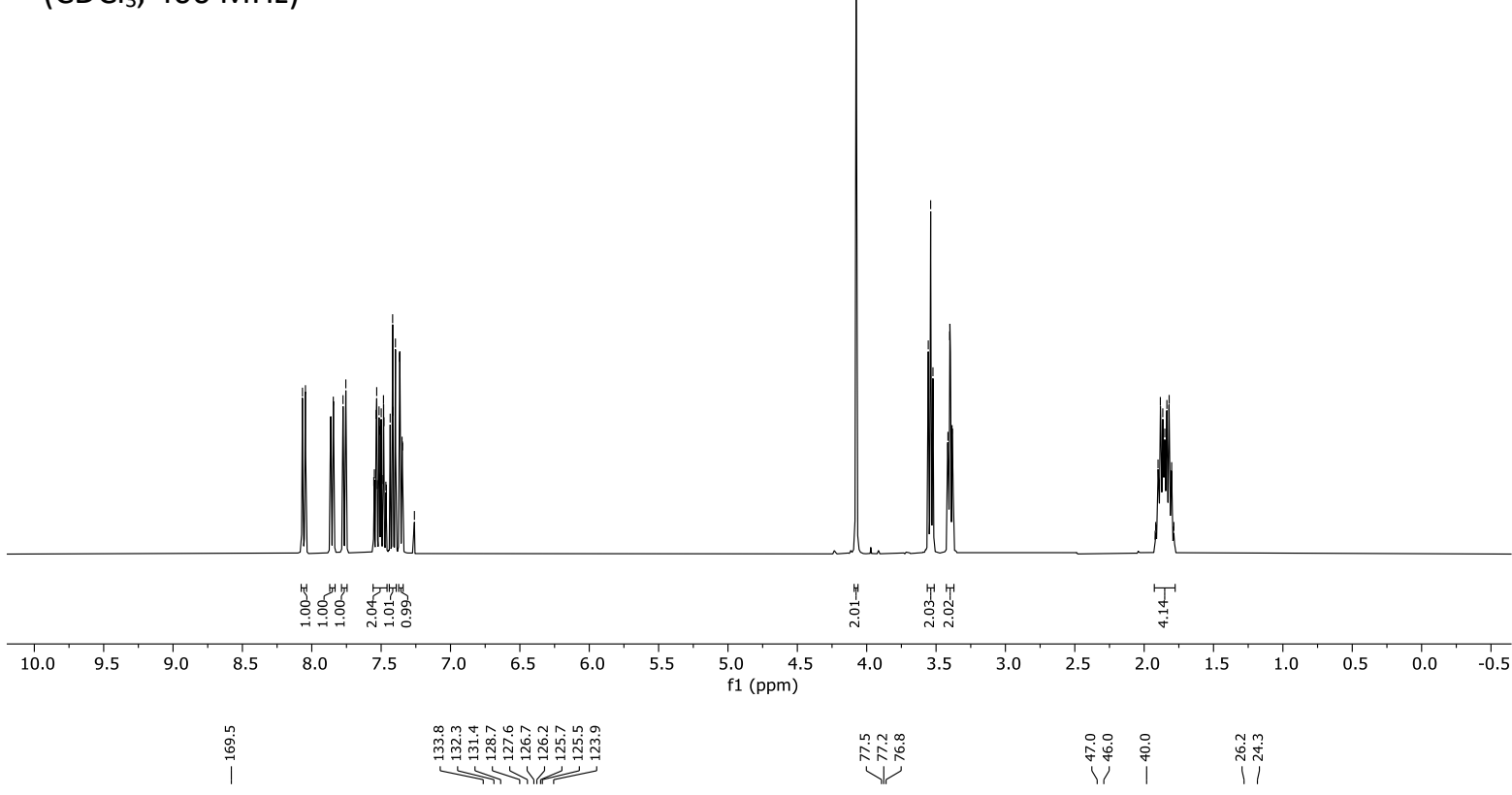

$\left(\mathrm{CDCl}_{3}, 100 \mathrm{MHz}\right)$

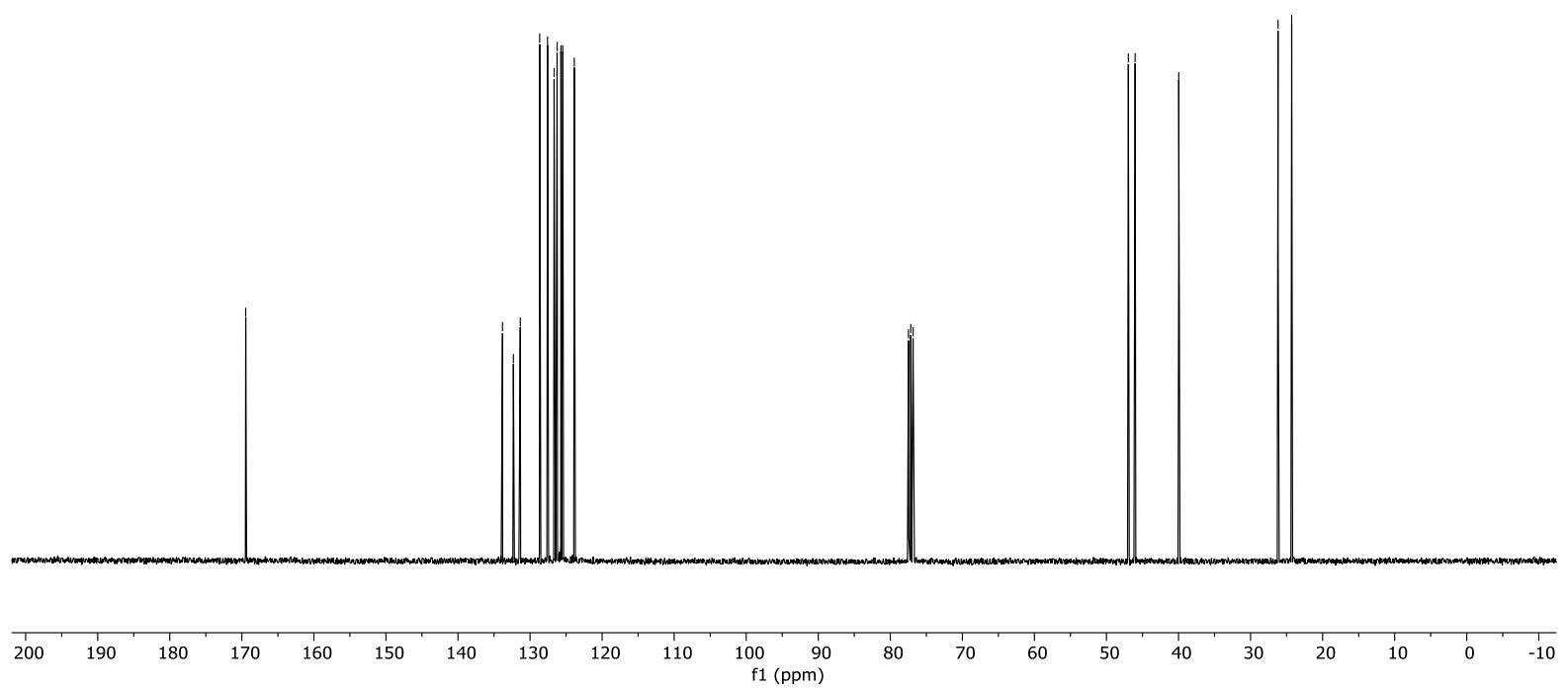




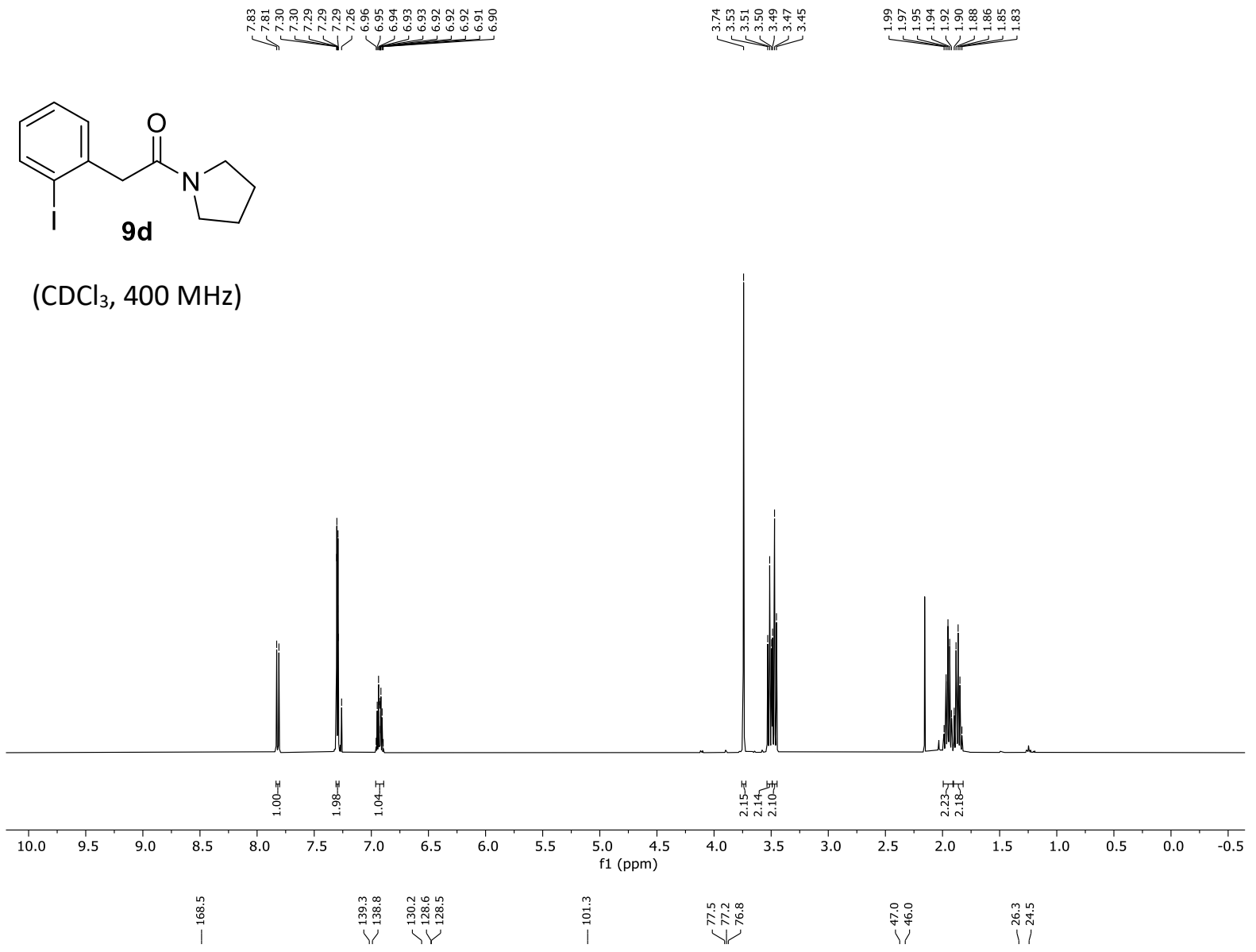

$\left(\mathrm{CDCl}_{3}, 100 \mathrm{MHz}\right)$

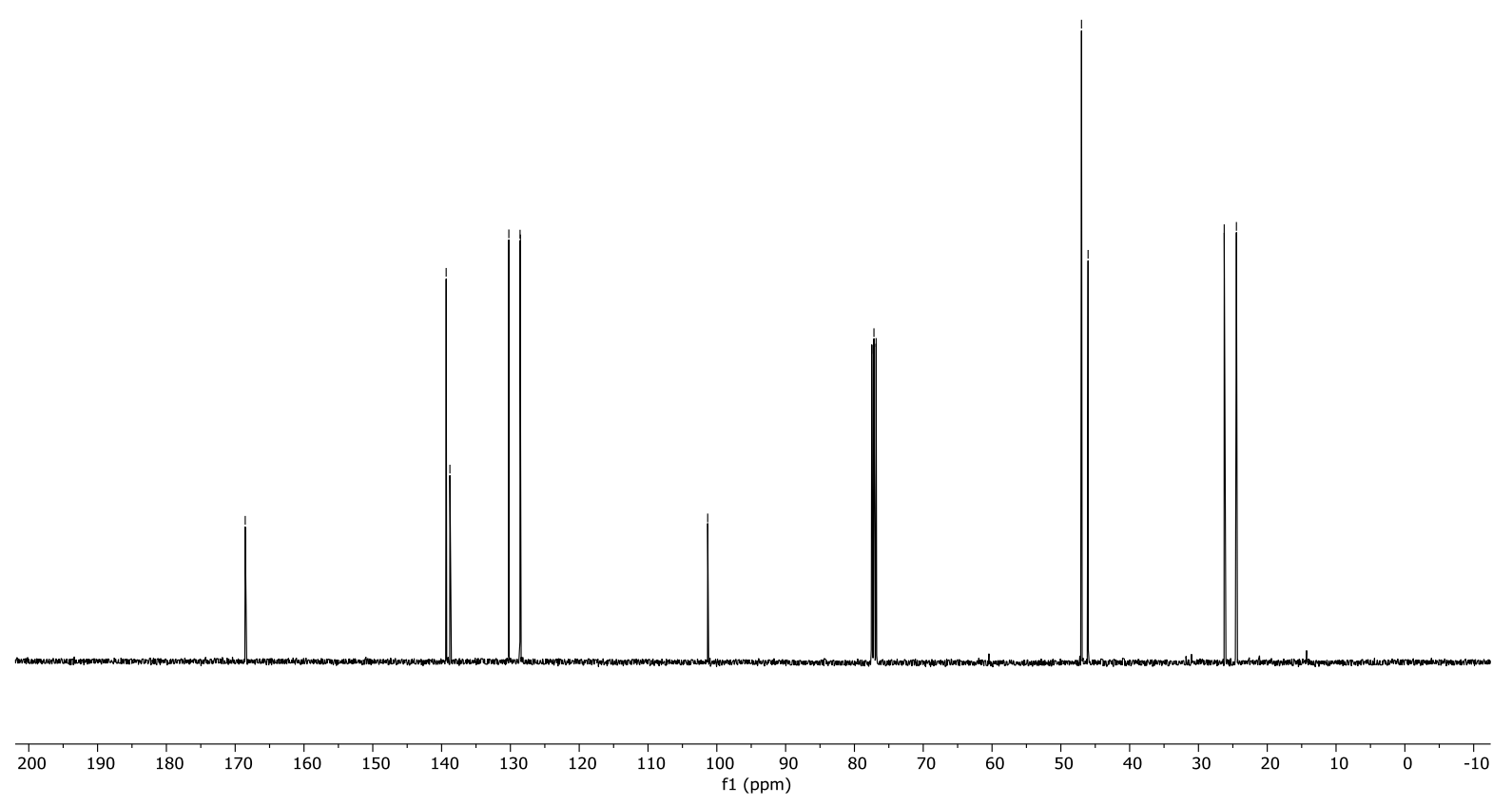



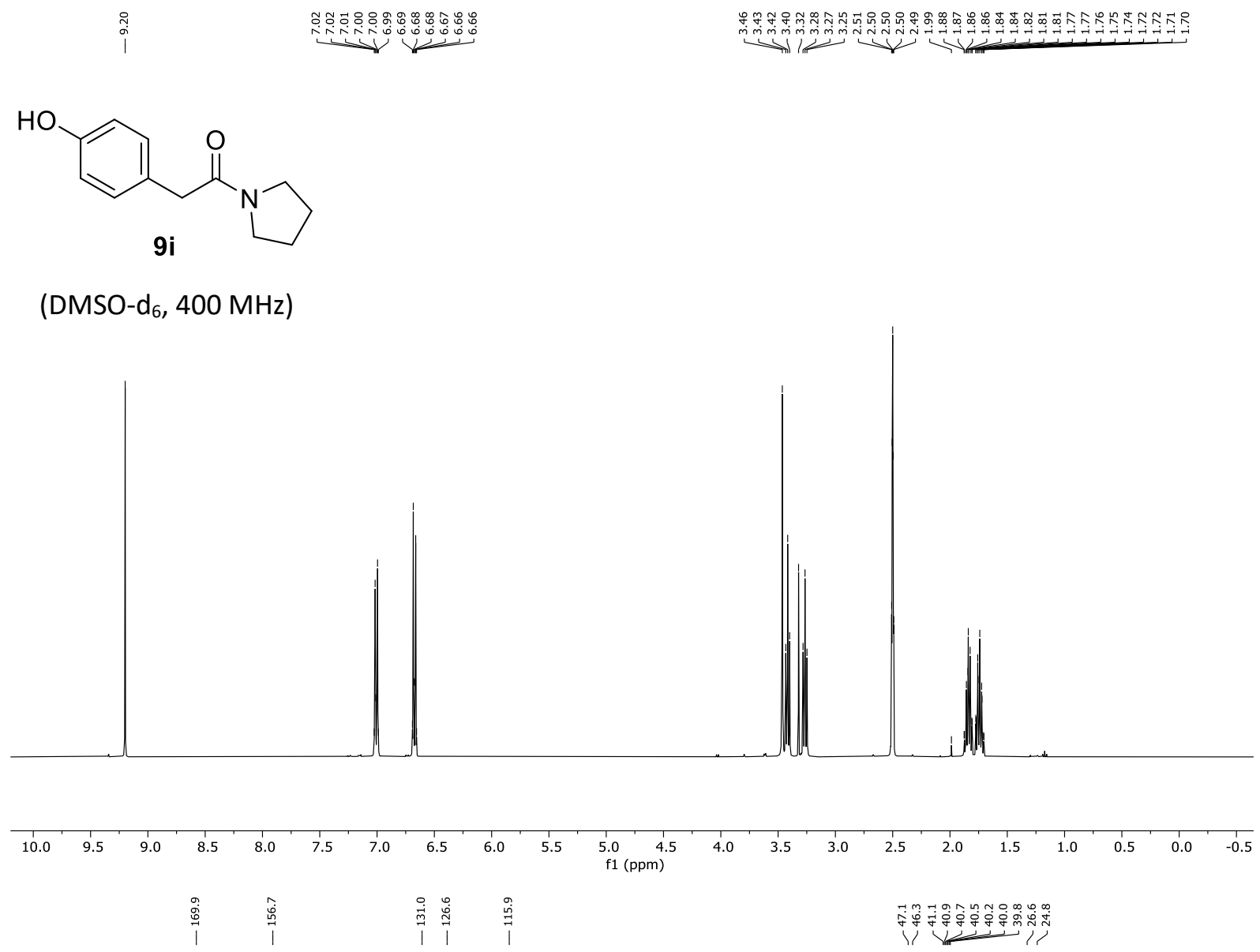

(DMSO-d $6,100 \mathrm{MHz}$ )

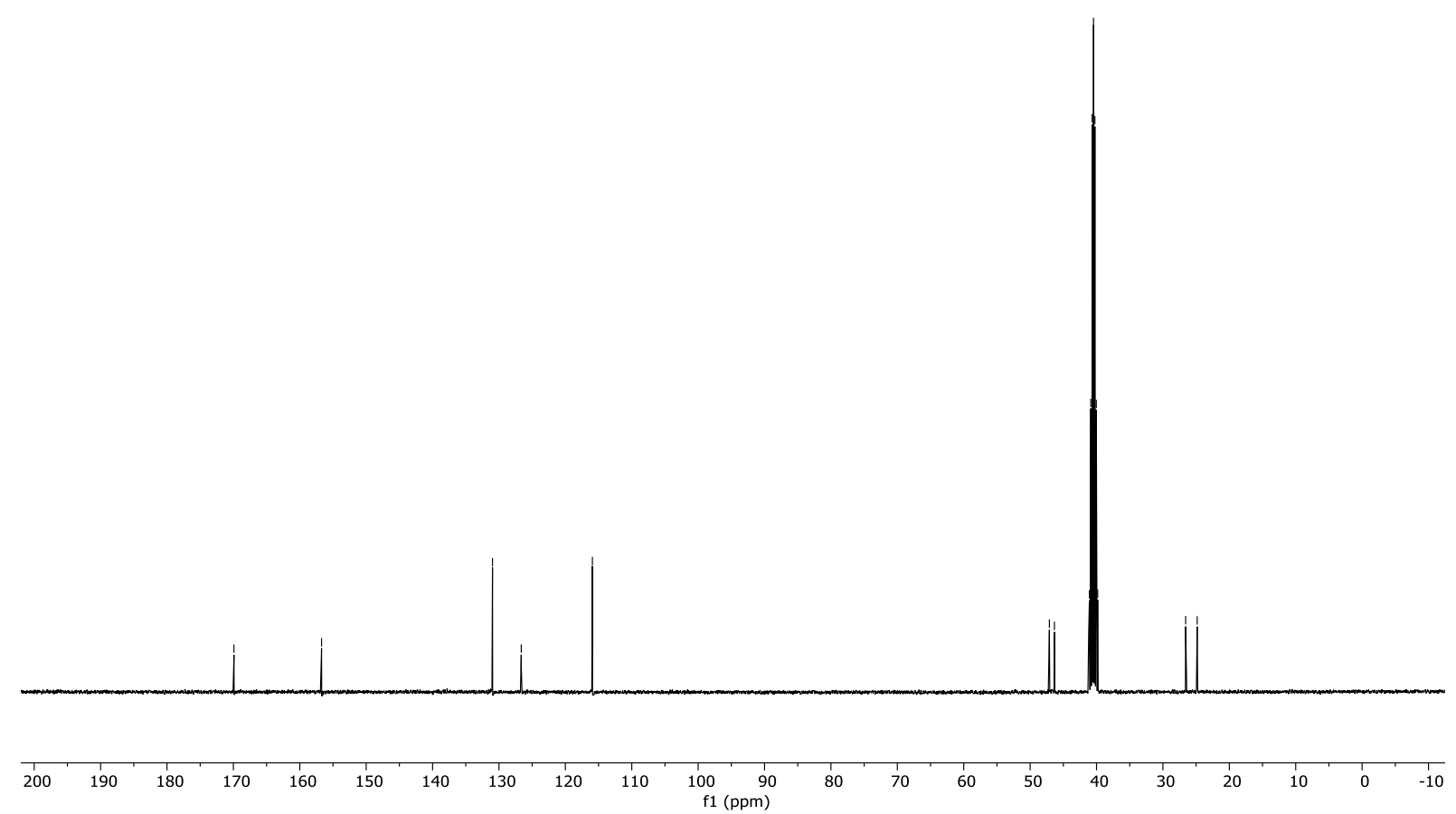




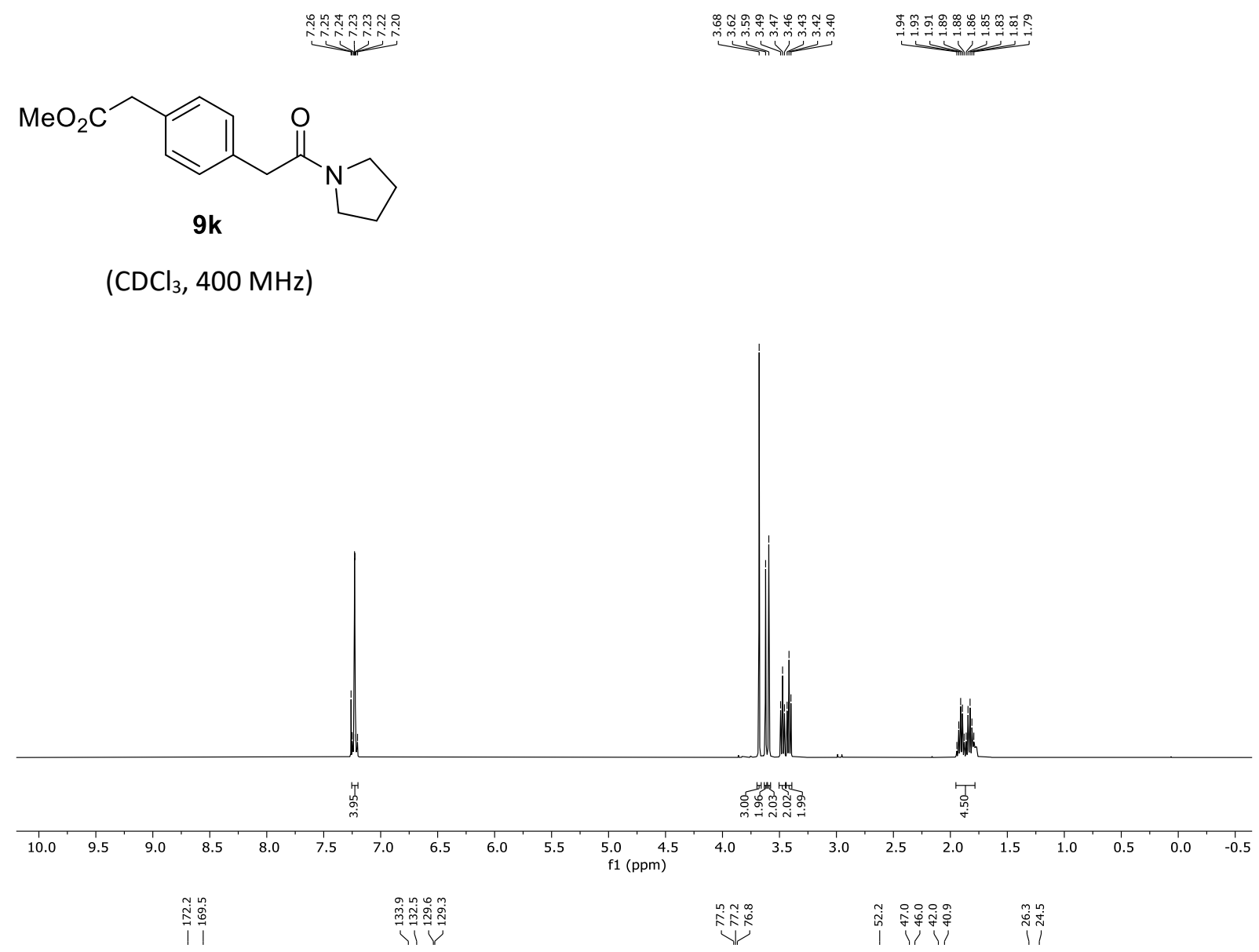

$\left(\mathrm{CDCl}_{3}, 100 \mathrm{MHz}\right)$

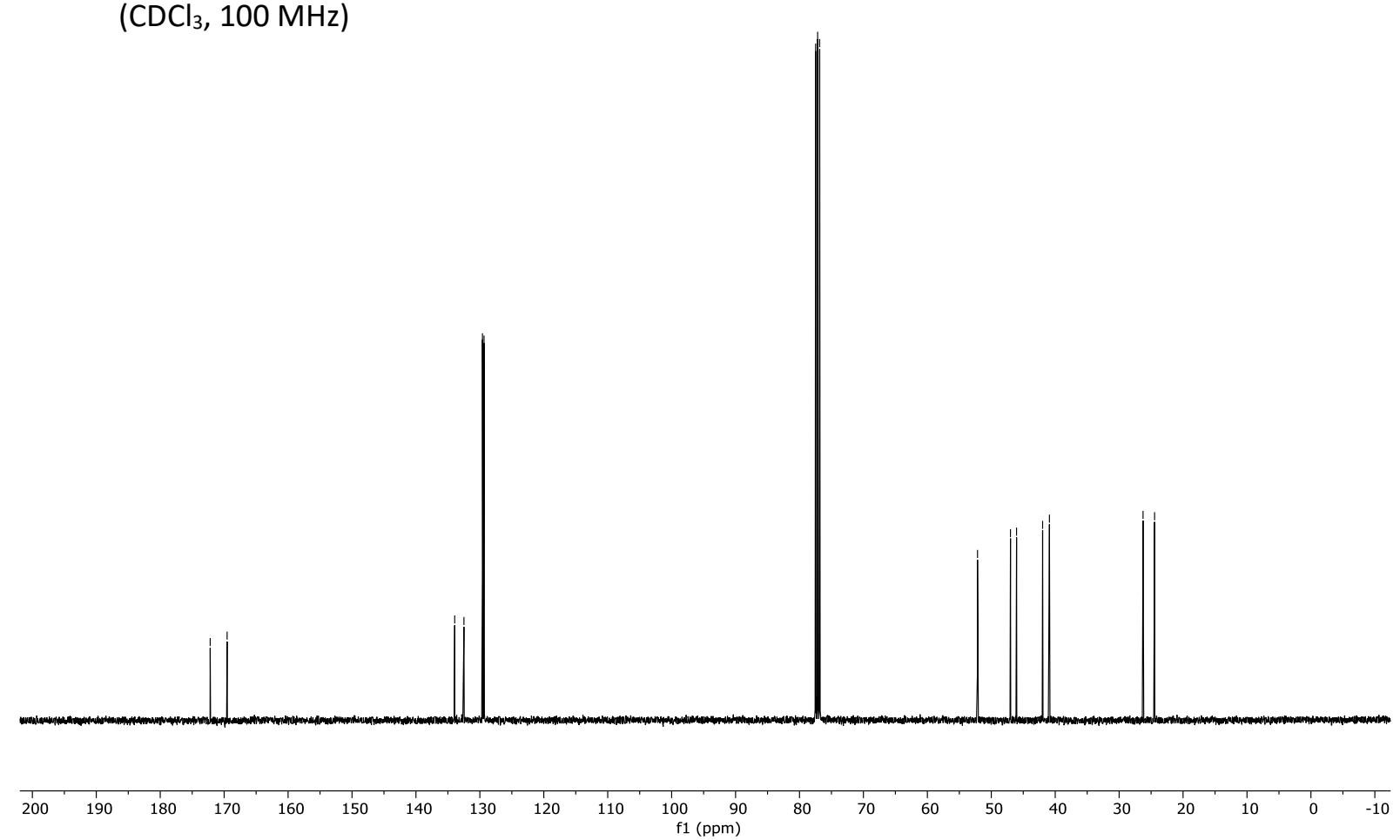




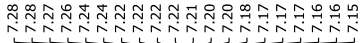<smiles>O=C(Cc1cccc(Cl)c1)N1CCCC1</smiles>

$\left(\mathrm{CDCl}_{3}, 400 \mathrm{MHz}\right)$

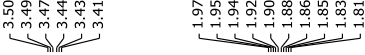

促
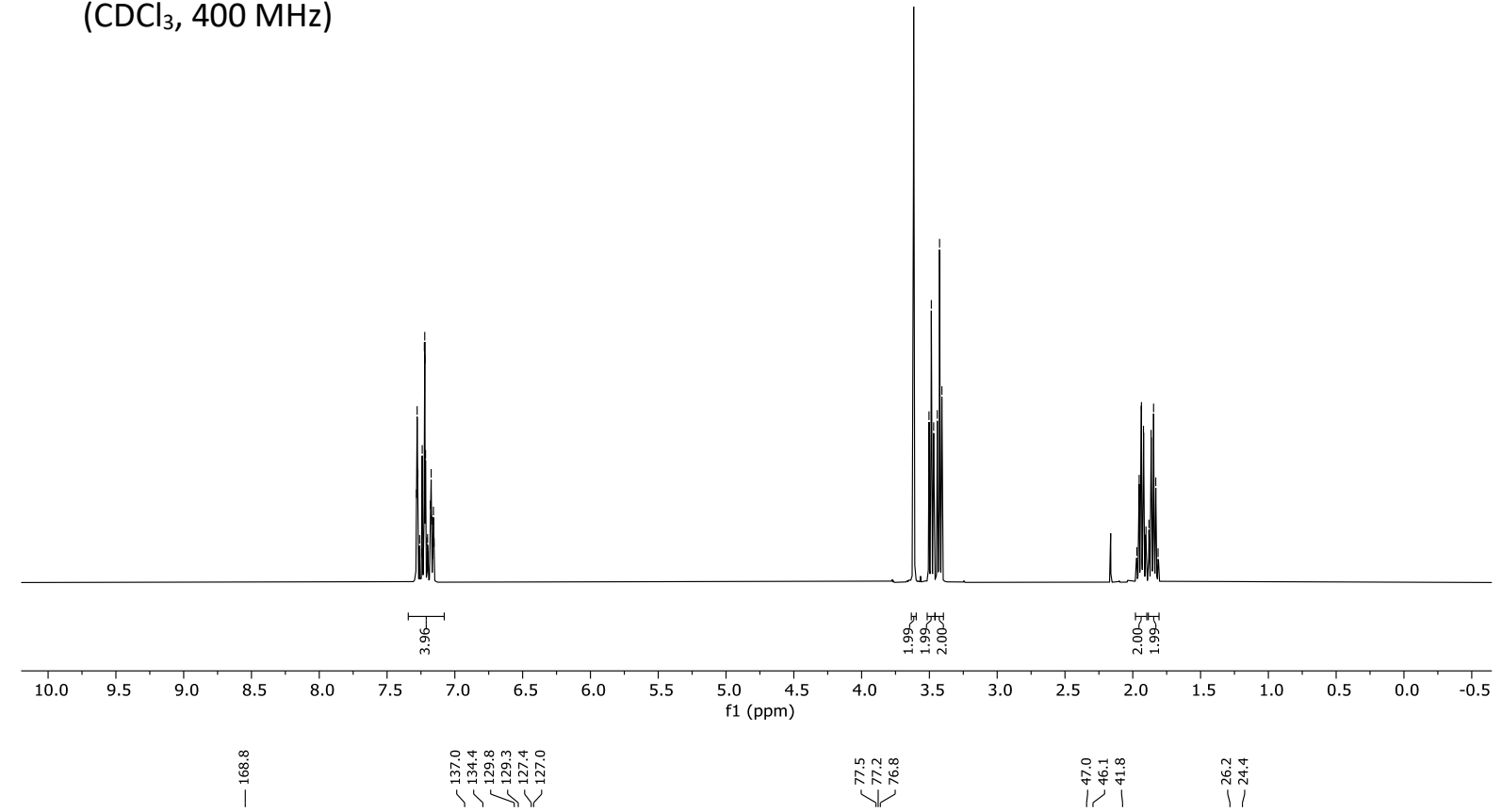

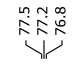

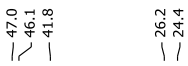

$\left(\mathrm{CDCl}_{3}, 100 \mathrm{MHz}\right)$

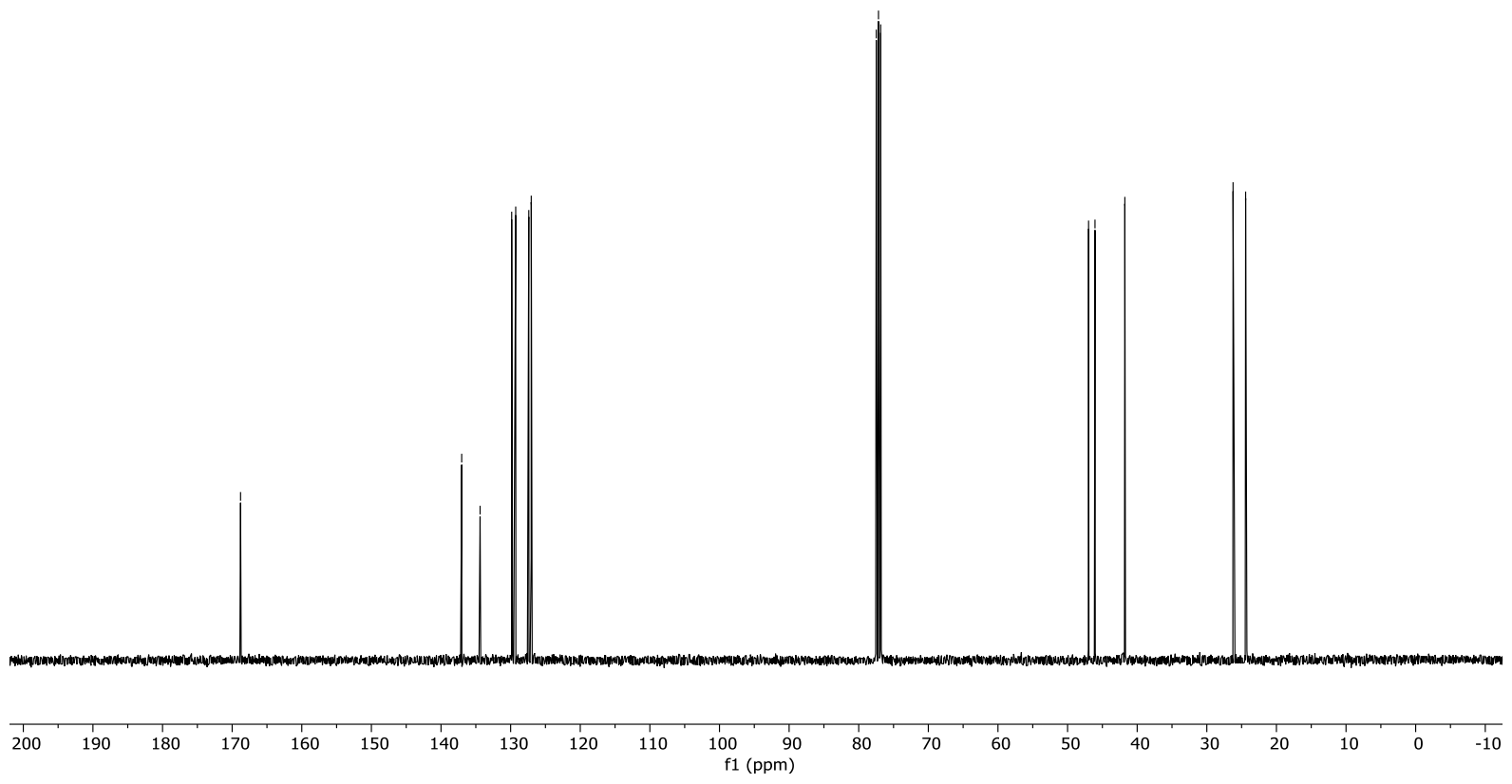



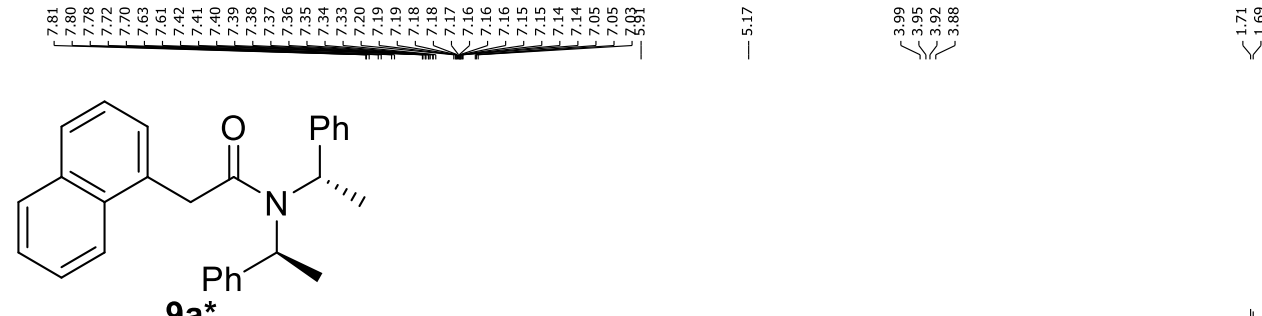

9a*

(tetrachloroethane- $\mathrm{d}_{2}$,

$80^{\circ} \mathrm{C}, 400 \mathrm{MHz}$ )
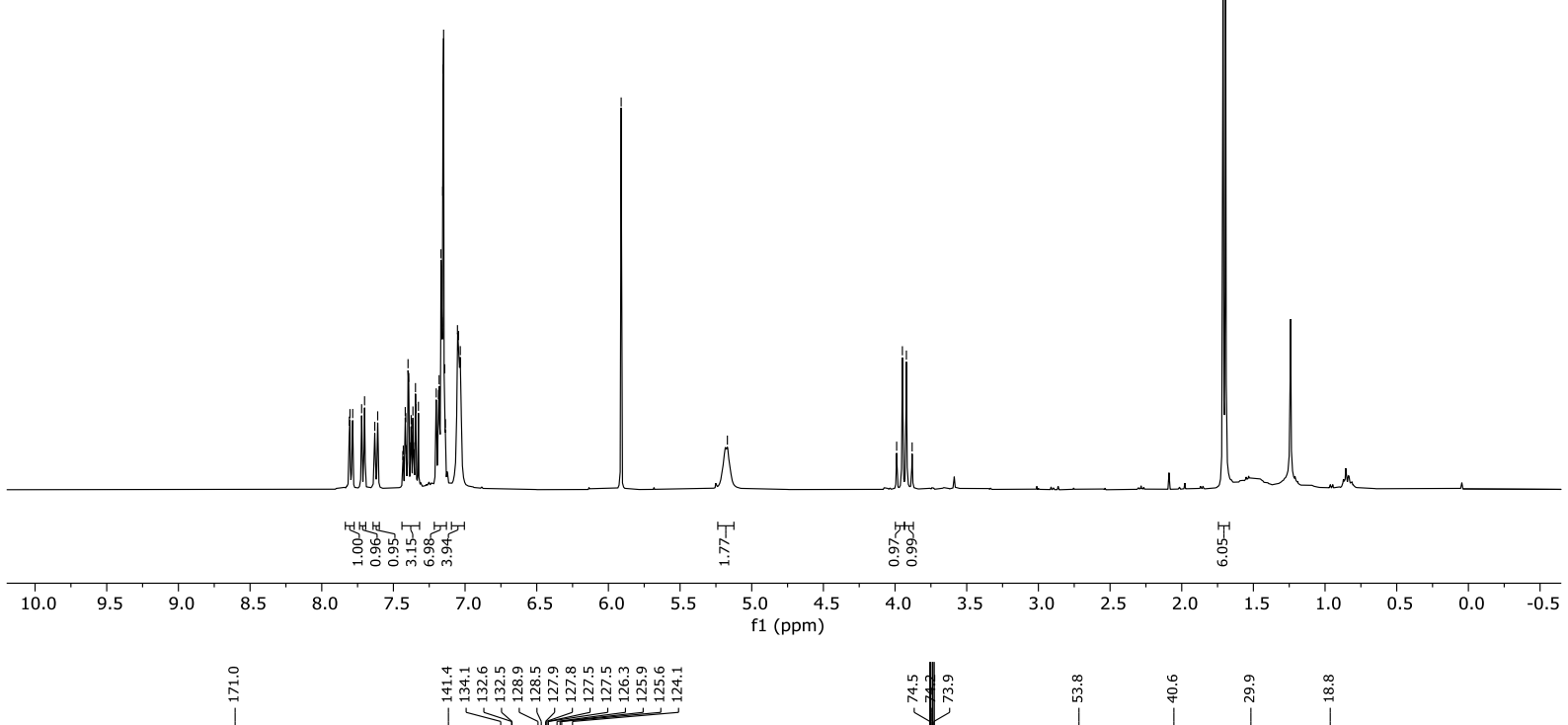

(tetrachloroethane- $d_{2}$,

$80^{\circ} \mathrm{C}, 100 \mathrm{MHz}$ )

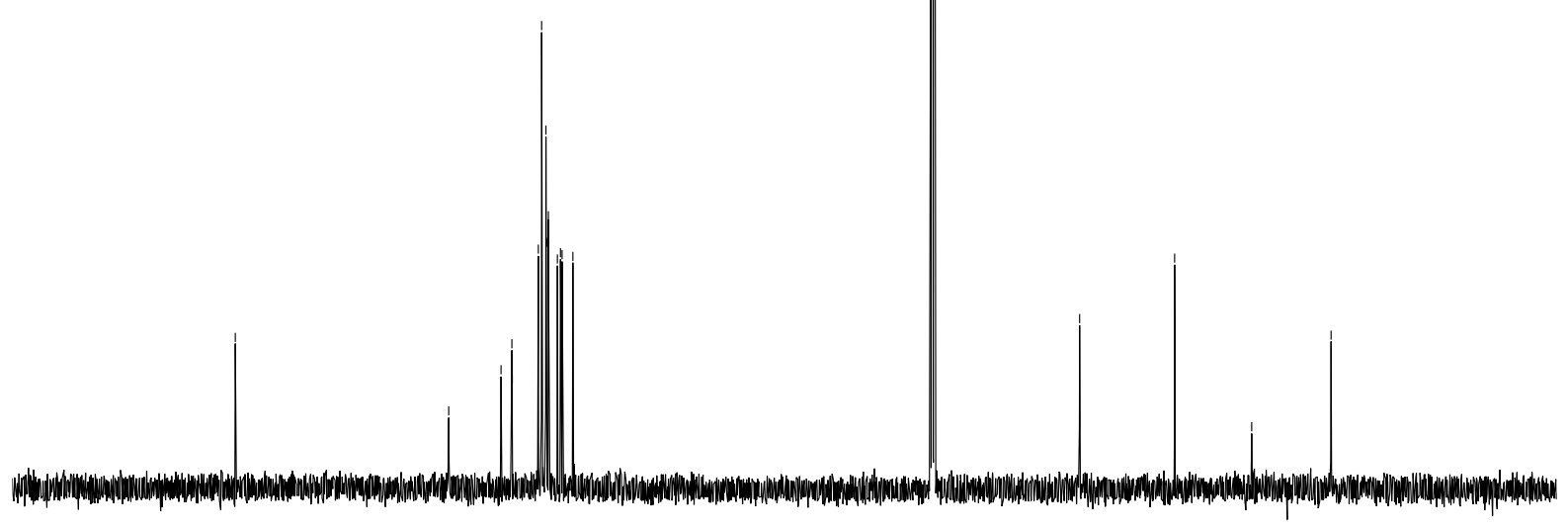

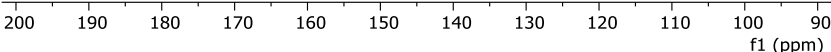




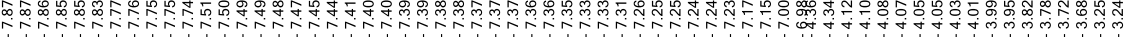<smiles>CO[C@H](c1ccccc1)[C@H](C)N(C)C(=O)Cc1cccc2ccccc12</smiles>

$\left(\mathrm{CDCl}_{3}, 400 \mathrm{MHz}\right)$

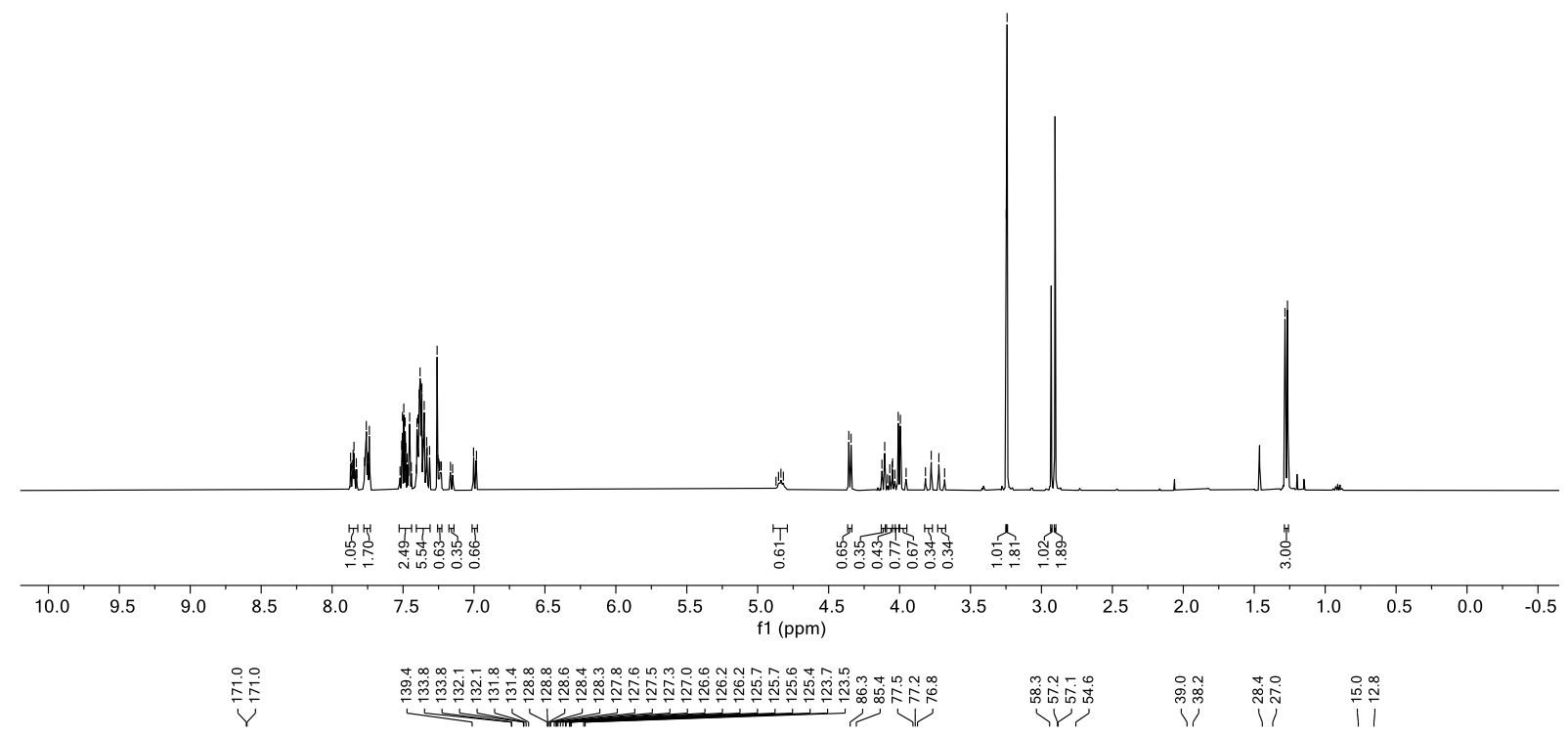

$\left(\mathrm{CDCl}_{3}, 100 \mathrm{MHz}\right)$

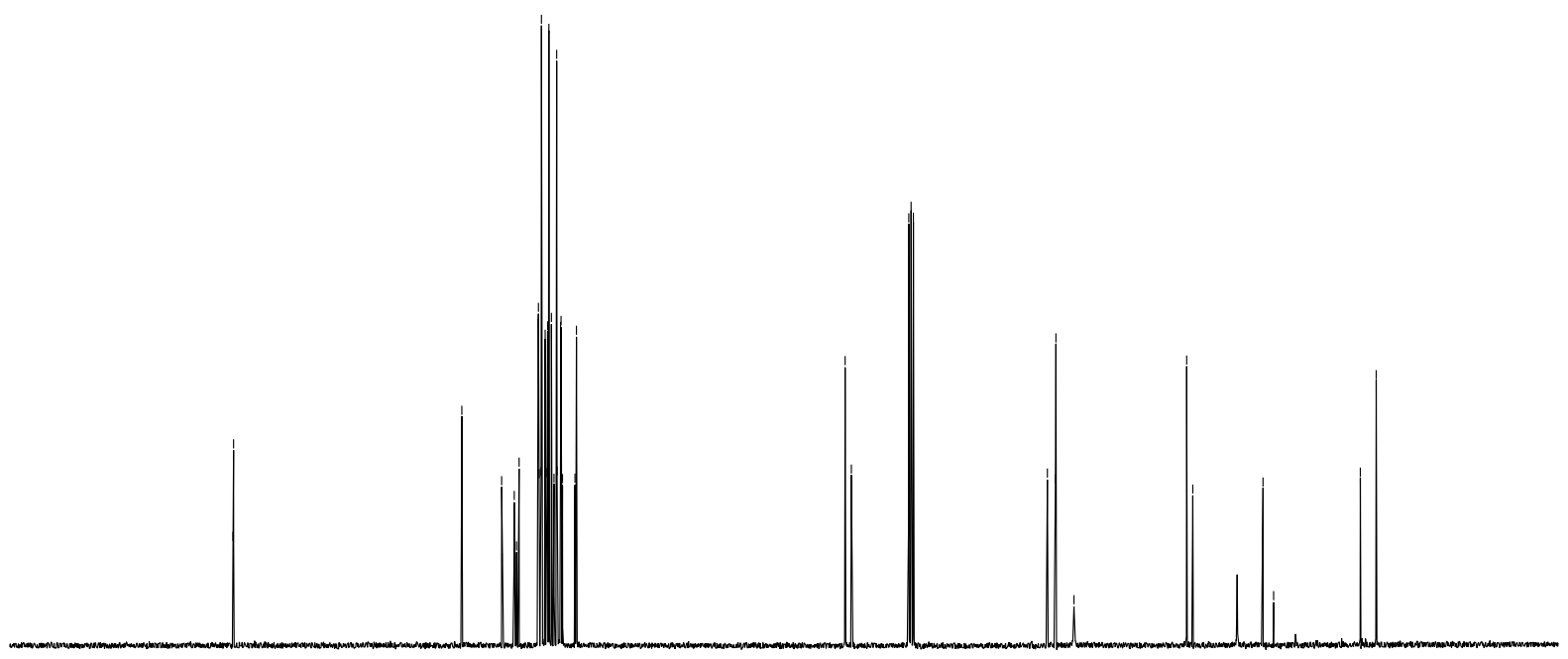

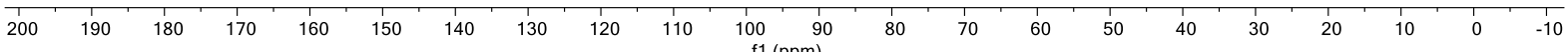




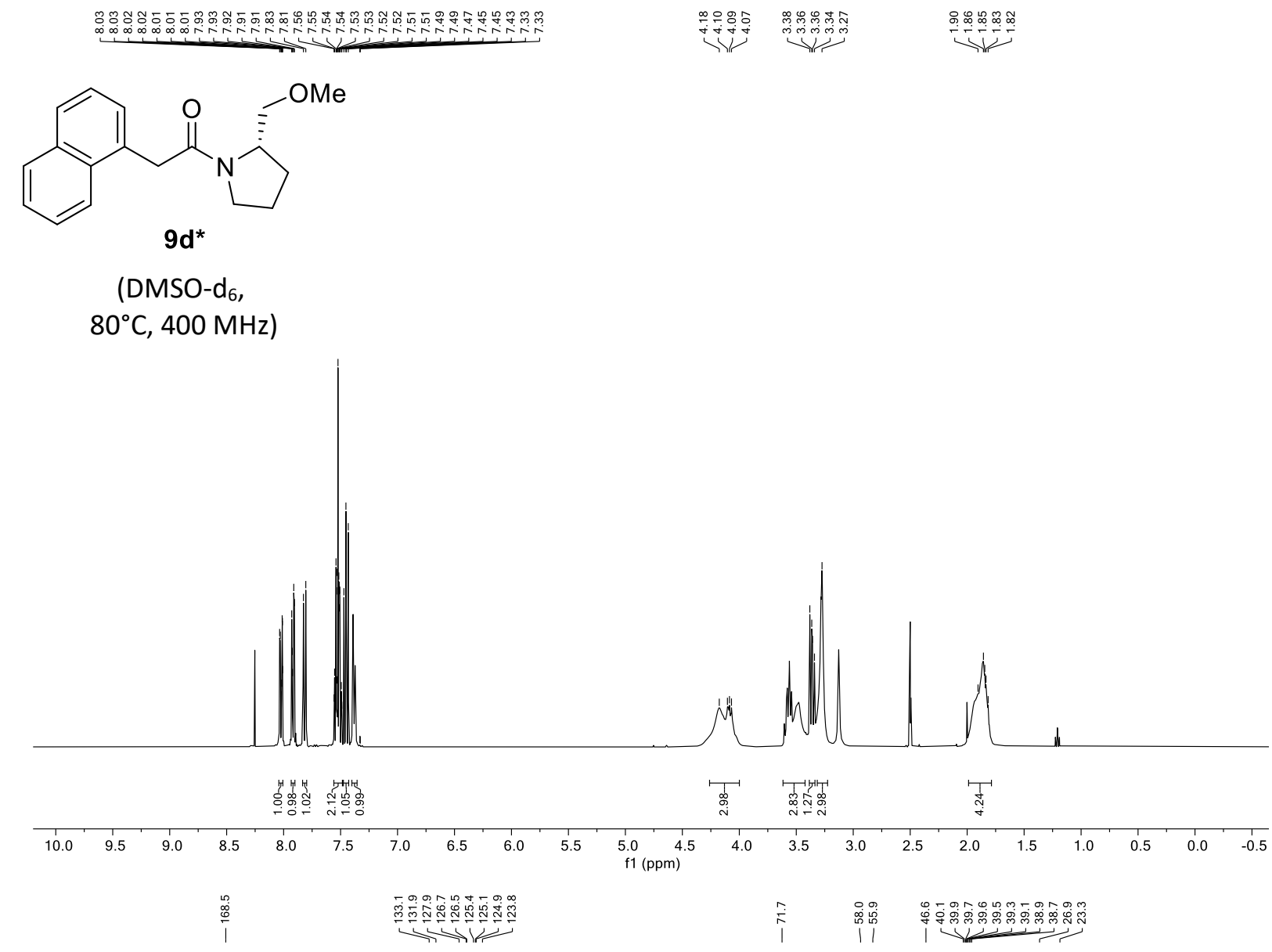

(DMSO-d $\mathrm{d}_{6}$ $80^{\circ} \mathrm{C}, 100 \mathrm{MHz}$ )

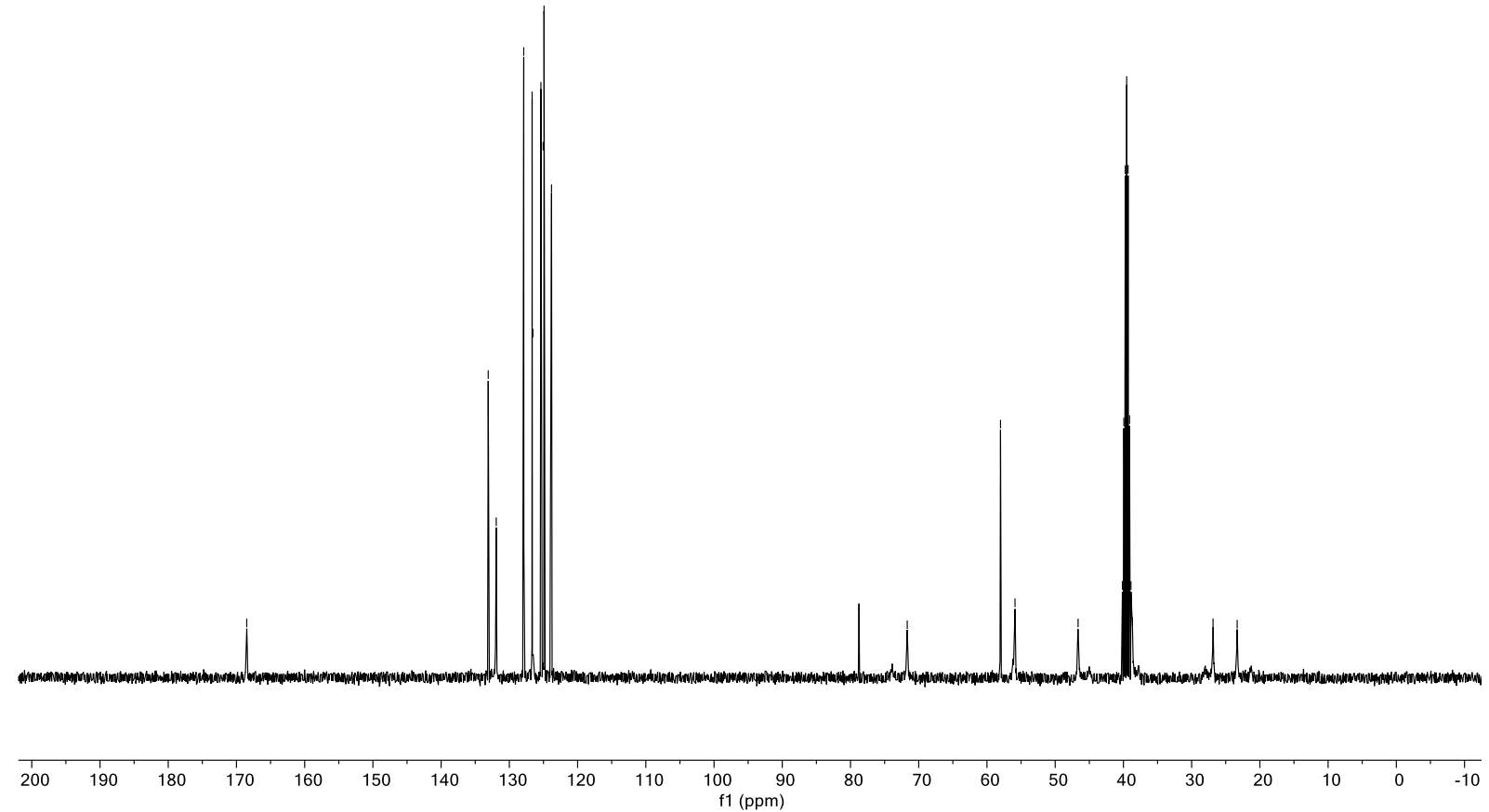




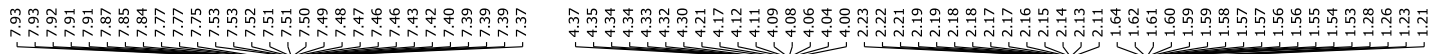<smiles>C[C@H]1CC[C@@H](C)N1C(=O)Cc1cccc2ccccc12</smiles>

$\left(\mathrm{CDCl}_{3}, 400 \mathrm{MHz}\right)$
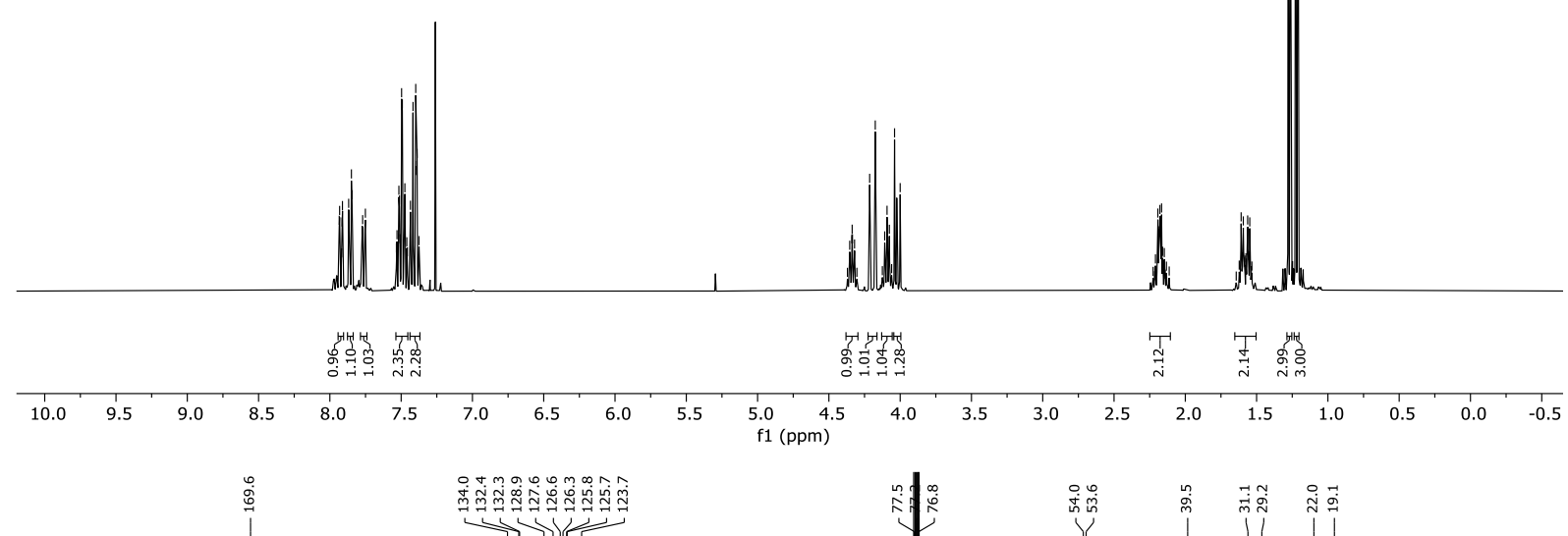

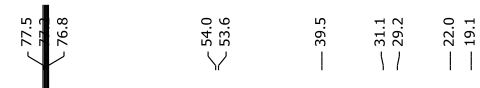

$\left(\mathrm{CDCl}_{3}, 100 \mathrm{MHz}\right)$

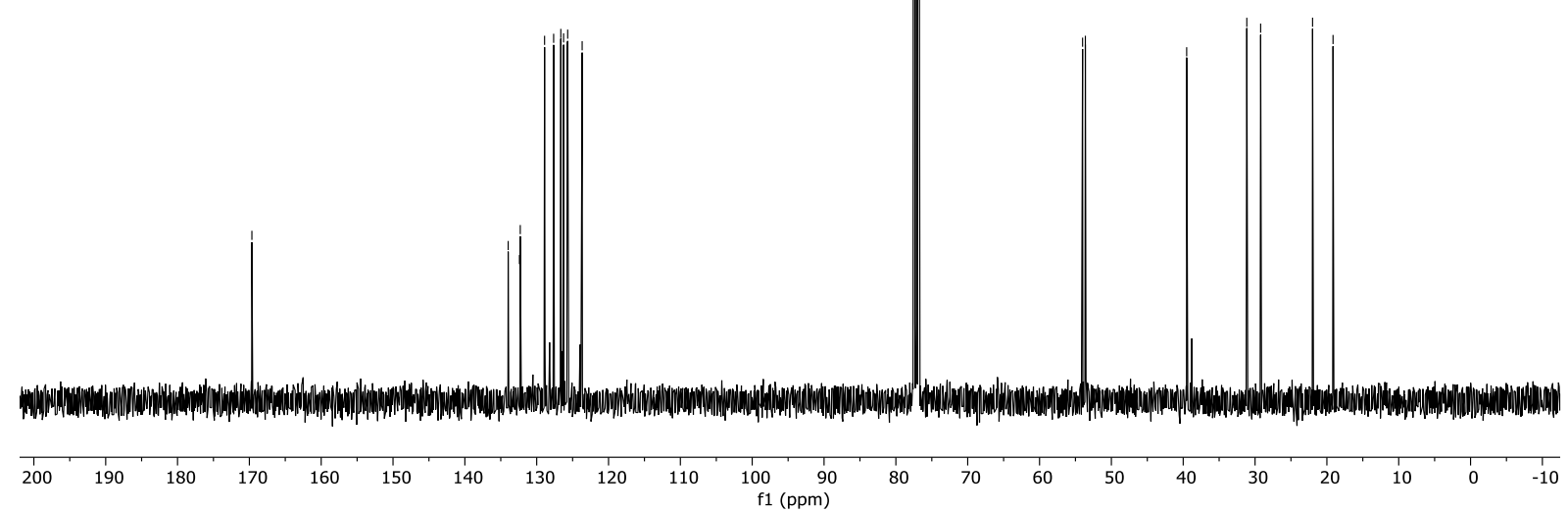




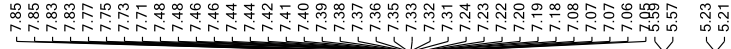<smiles>O=C(Cc1cccc2ccccc12)N1[C@H](c2ccccc2)CC[C@H]1c1ccccc1</smiles>

$\left(\mathrm{CDCl}_{3}, 400 \mathrm{MHz}\right)$ $\underbrace{0.000}$

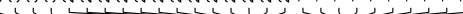

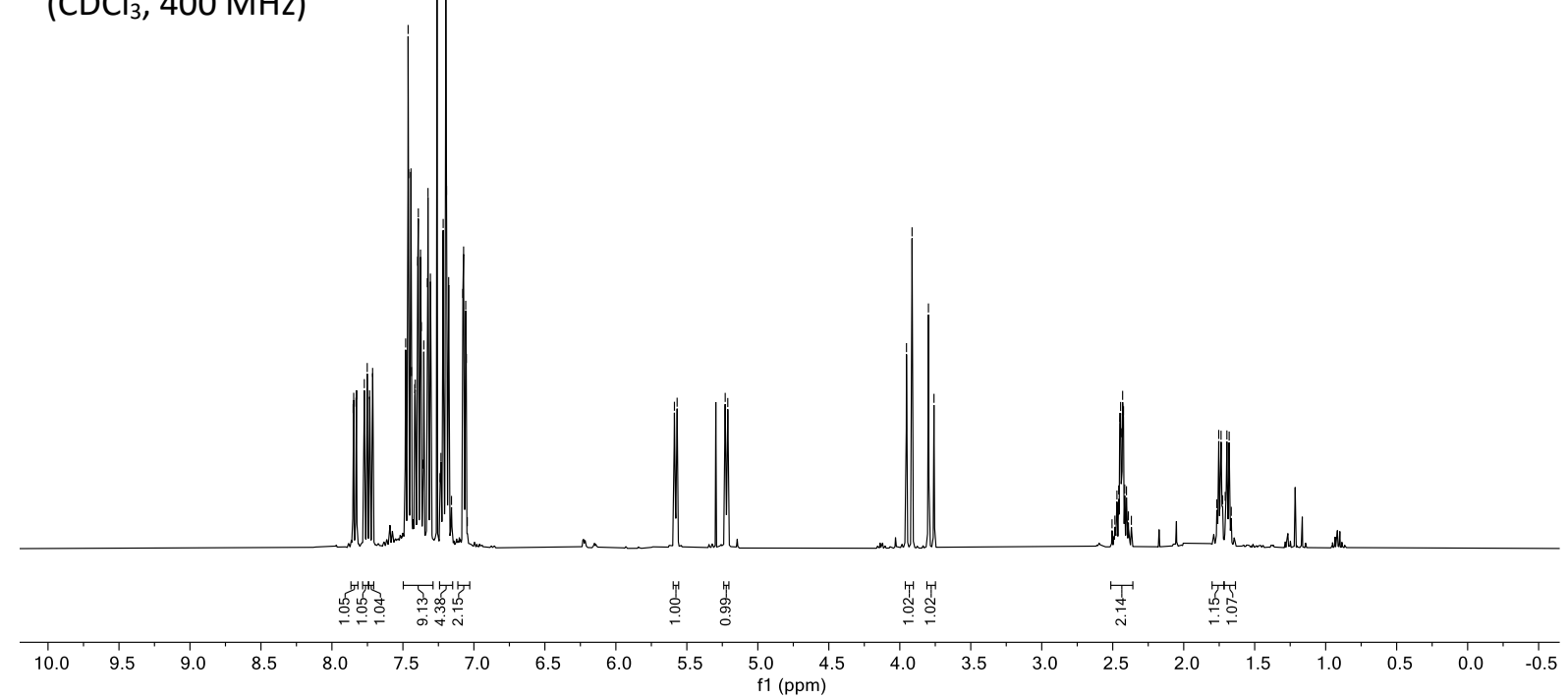

$\underbrace{1}_{\substack{0 \\ 0}}$

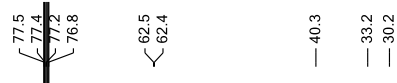

$\left(\mathrm{CDCl}_{3}, 100 \mathrm{MHz}\right)$

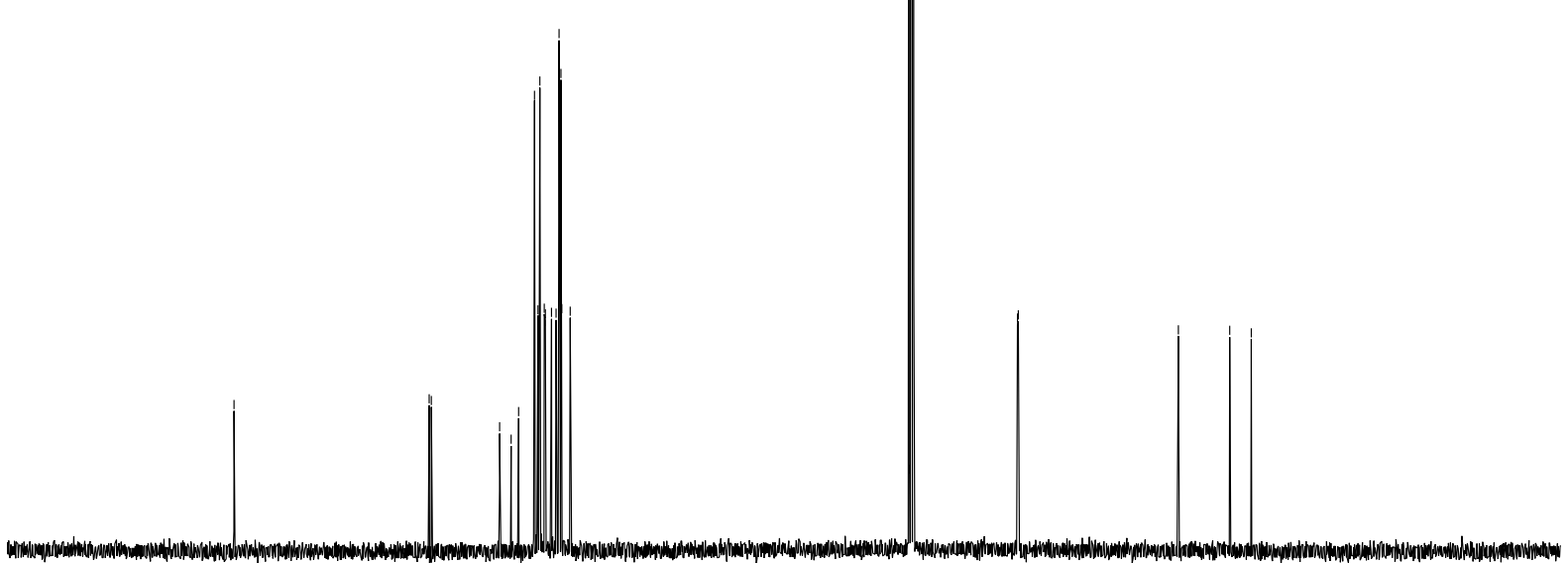

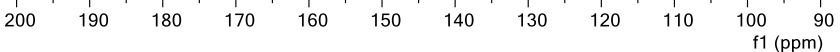




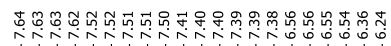

$\overbrace{F}^{O}$

$10 a$

$\left(\mathrm{CDCl}_{3}, 400 \mathrm{MHz}\right)$
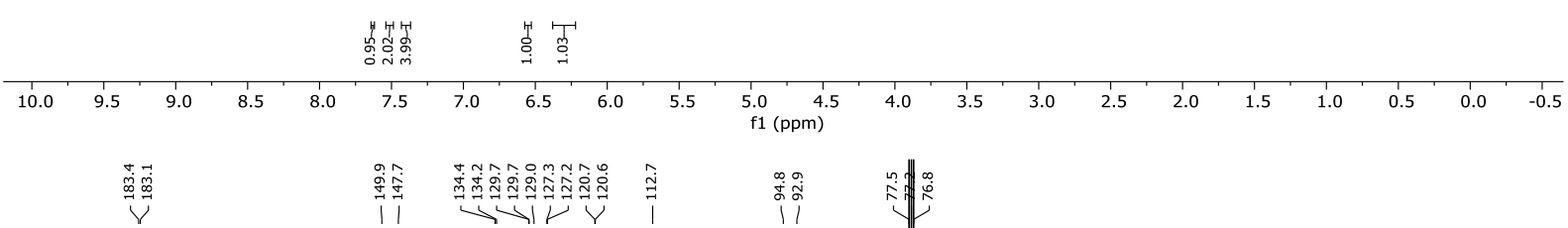

$\left(\mathrm{CDCl}_{3}, 100 \mathrm{MHz}\right)$

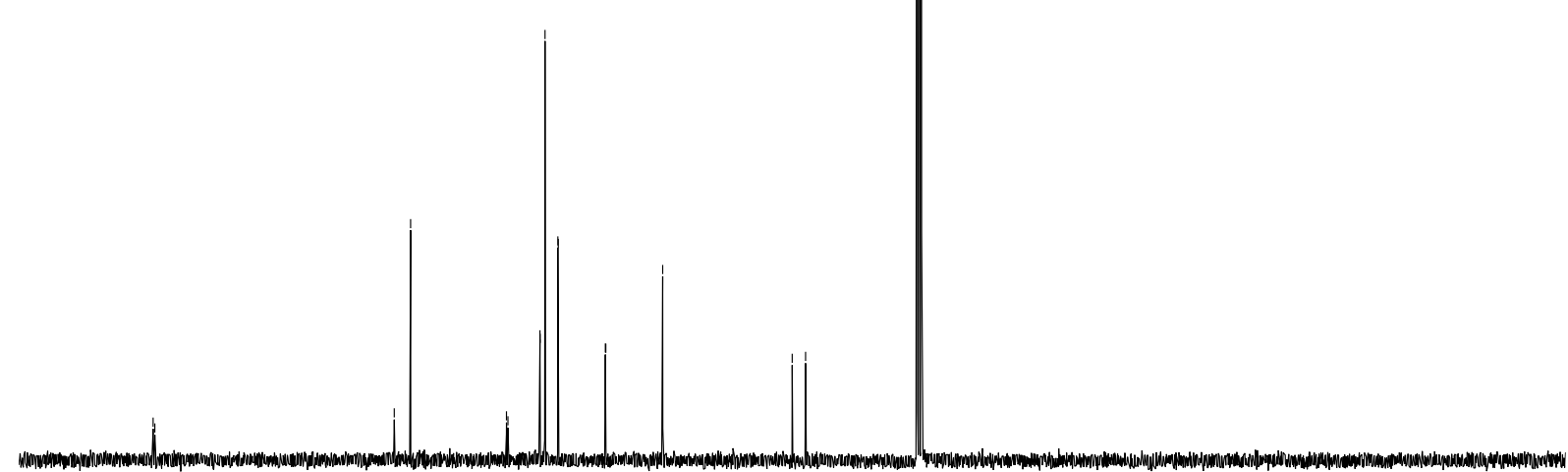


Divergent Synthesis of $\alpha$-Fluorinated Carbonyl and Carboxyl Derivatives by Double Electrophilic Activation of Amides

standard (fluorobenzene)

$\left(\mathrm{CDCl}_{3}, 376 \mathrm{MHz}\right)$
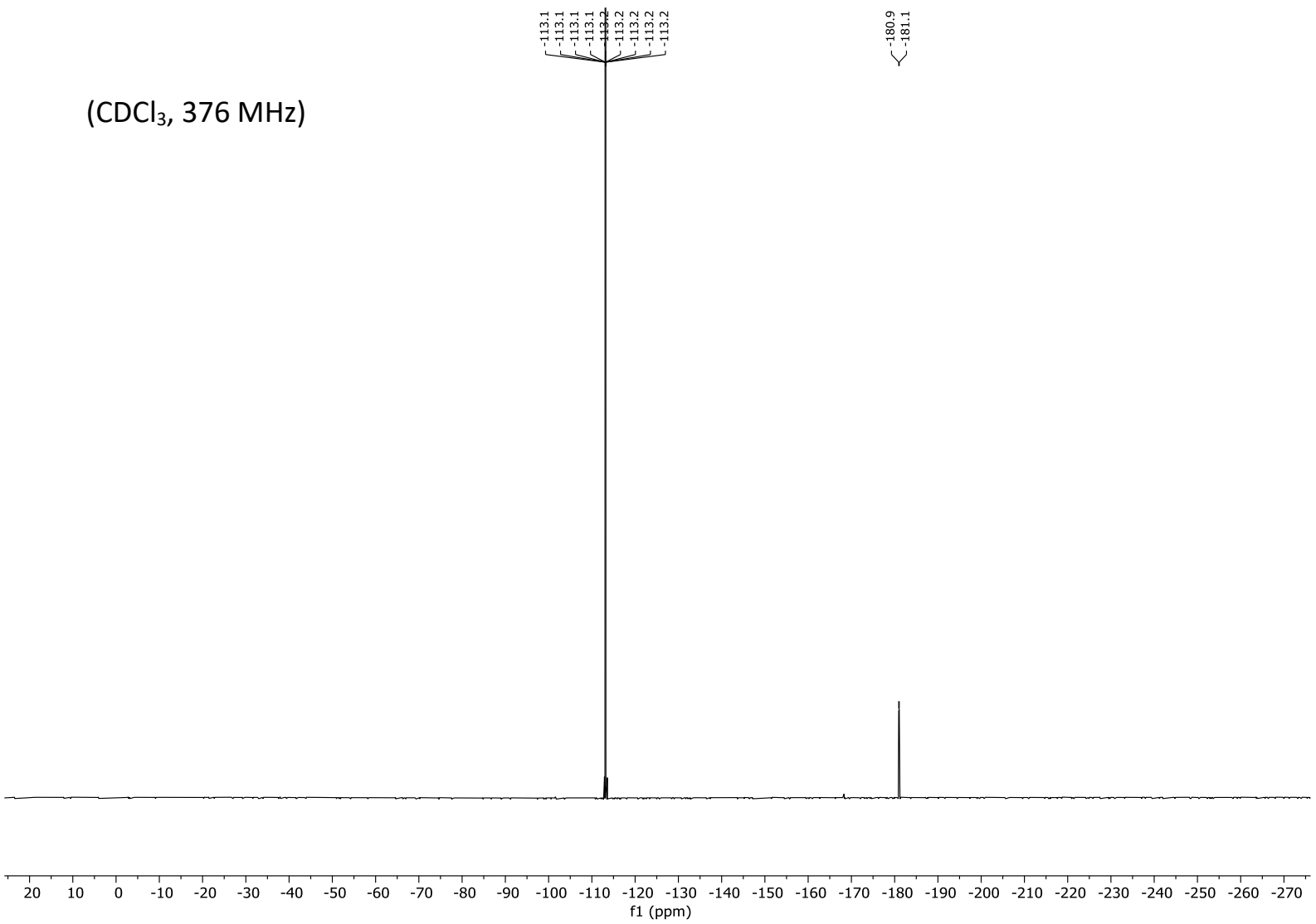


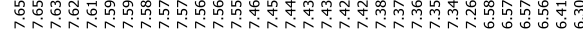

$\mathrm{Ph}$<smiles>O=C(c1ccco1)C(F)c1ccccc1</smiles>

$10 b$

$\left(\mathrm{CDCl}_{3}, 400 \mathrm{MHz}\right)$

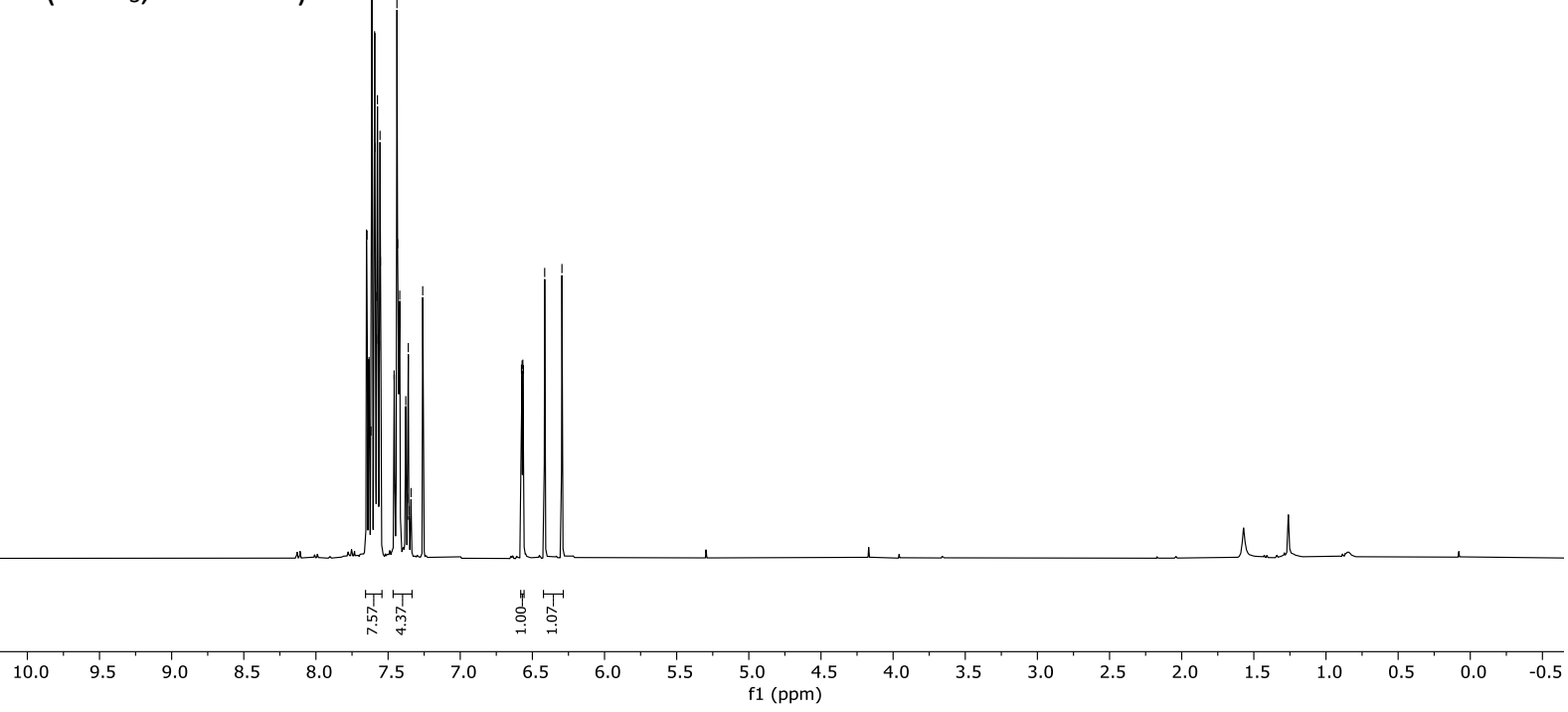

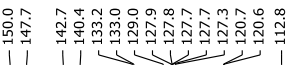

$\left(\mathrm{CDCl}_{3}, 100 \mathrm{MHz}\right)$

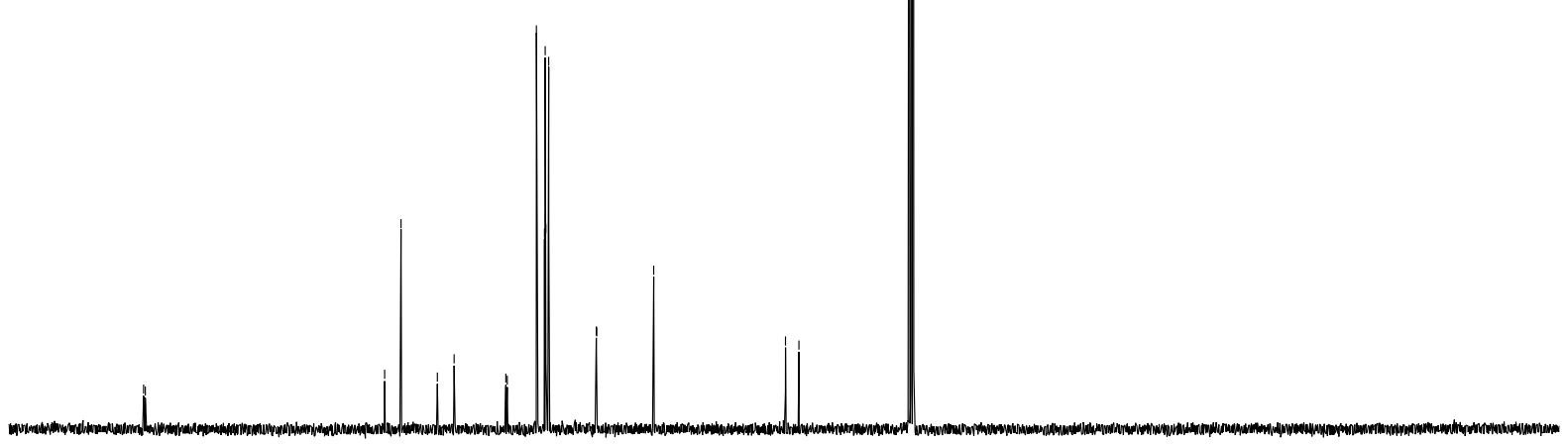

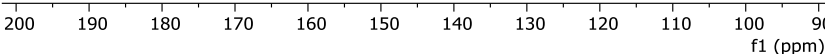


Divergent Synthesis of $\alpha$-Fluorinated Carbonyl and Carboxyl Derivatives by Double Electrophilic Activation of Amides

$\left(\mathrm{CDCl}_{3}, 376 \mathrm{MHz}\right)$

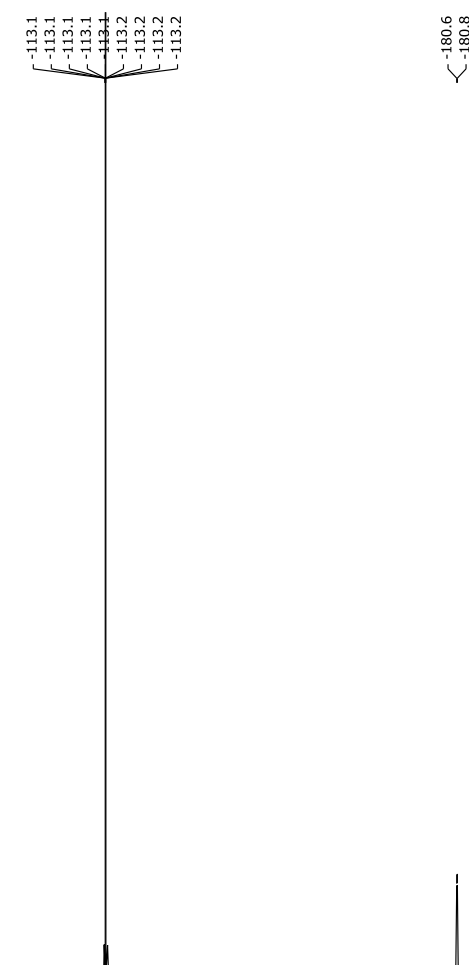

$\begin{array}{llllllllllllllllllllllllllllllllllll}20 & 10 & 0 & -10 & -20 & -30 & -40 & -50 & -60 & -70 & -80 & -90 & -100 & -110 & -120 & -130 & -140 & -150 & -160 & -170 & -180 & -190 & -200 & -210 & -220 & -230 & -240 & -250 & -260 & -270\end{array}$ f1 (ppm) 


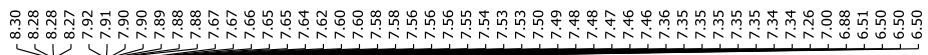

$\overbrace{1}^{C}$

$10 \mathrm{c}$

$\left(\mathrm{CDCl}_{3}, 400 \mathrm{MHz}\right)$

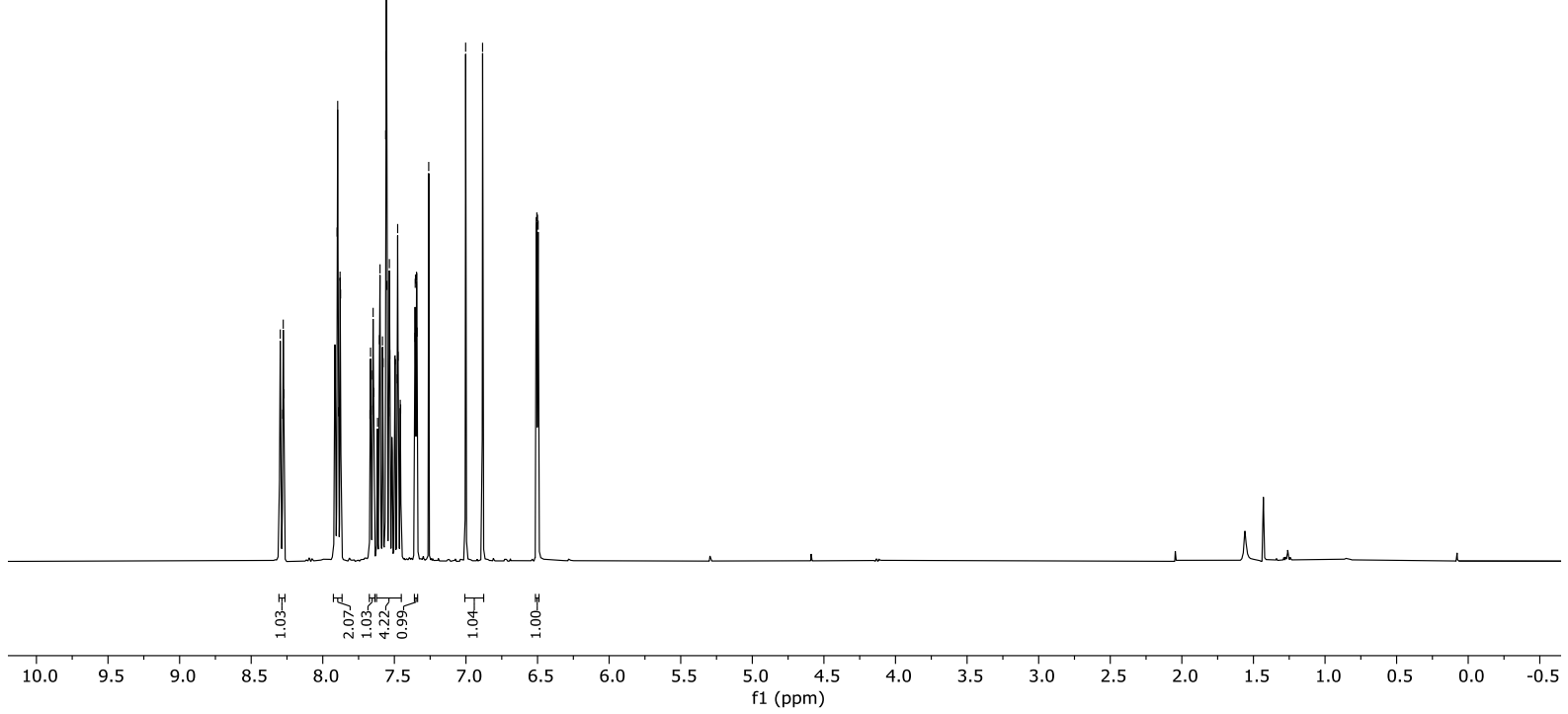

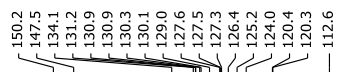

索早

$\left(\mathrm{CDCl}_{3}, 100 \mathrm{MHz}\right)$
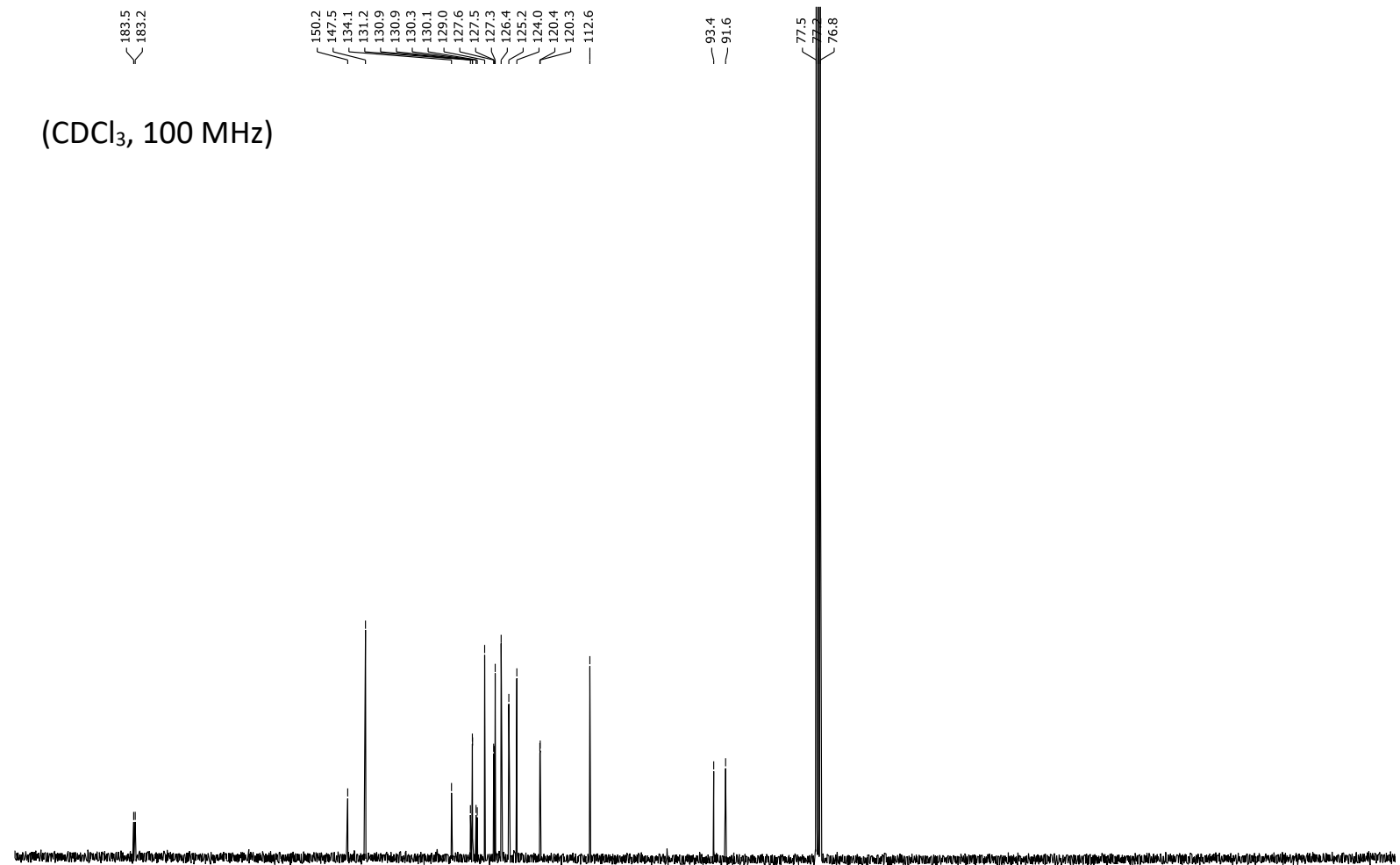

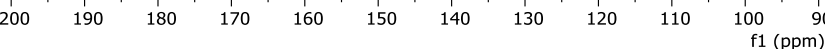


Divergent Synthesis of $\alpha$-Fluorinated Carbonyl and Carboxyl Derivatives by Double Electrophilic Activation of Amides Internal

standard (fluorobenzene)

$\left(\mathrm{CDCl}_{3}, 376 \mathrm{MHz}\right)$
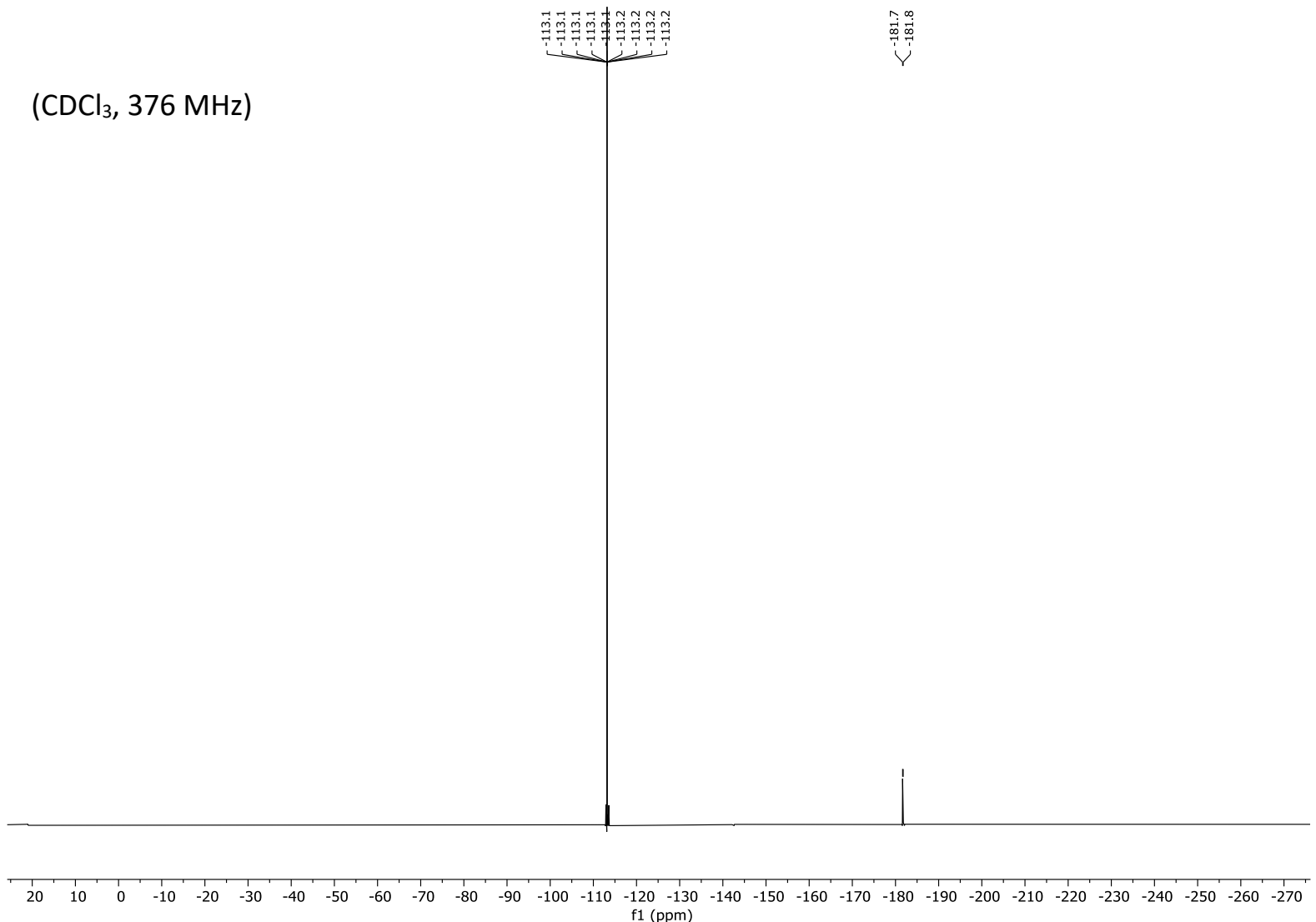


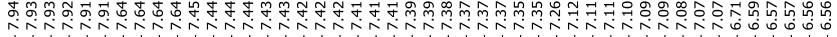<smiles>O=C(c1ccco1)C(F)c1ccccc1I</smiles>

$10 d$

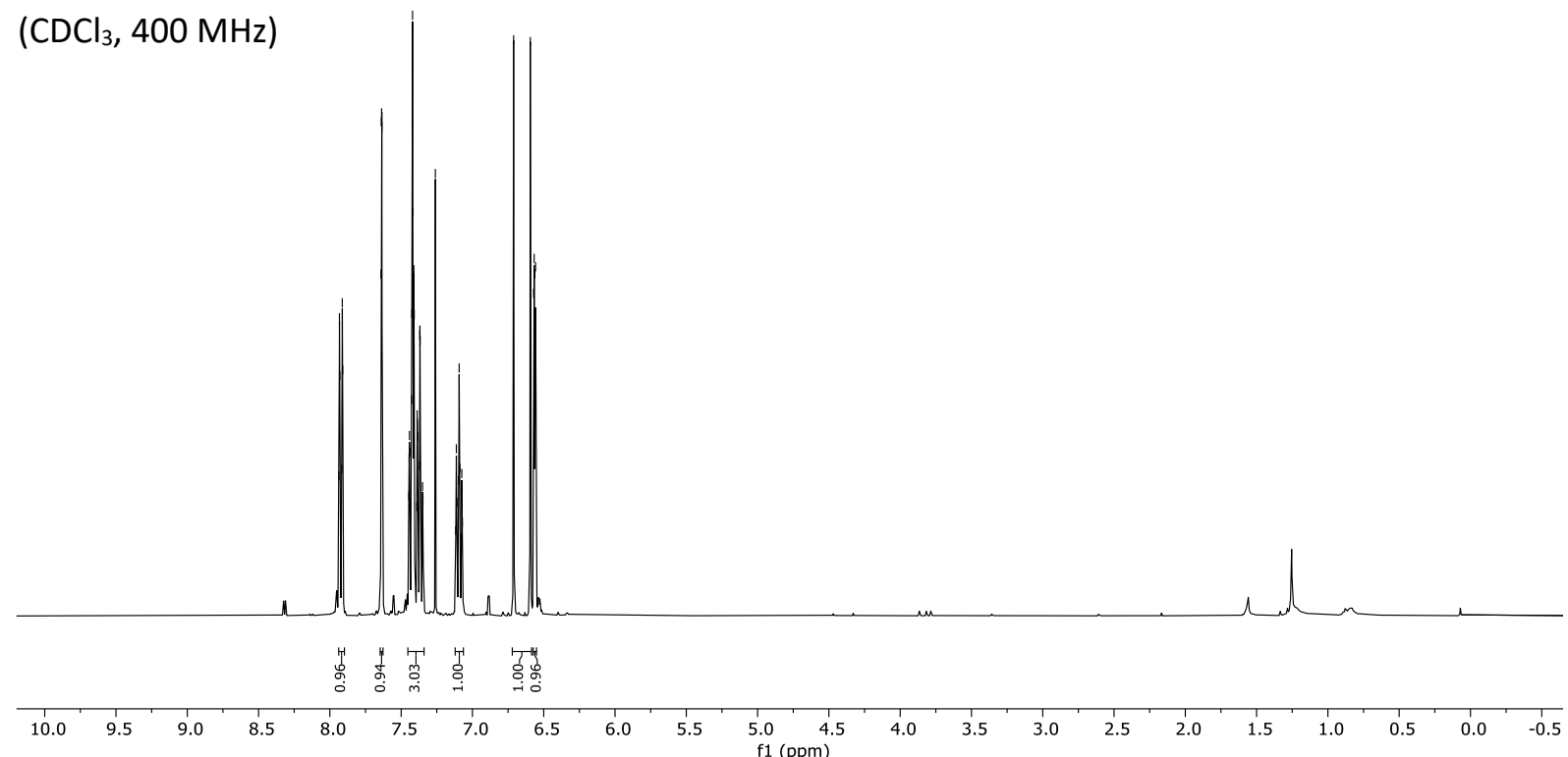

$\left(\mathrm{CDCl}_{3}, 100 \mathrm{MHz}\right)$

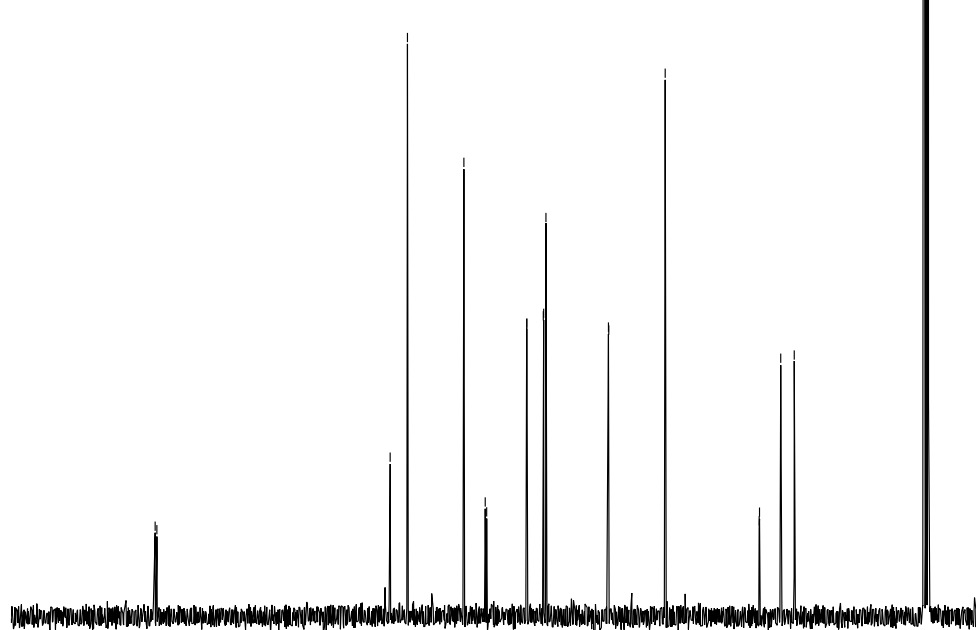

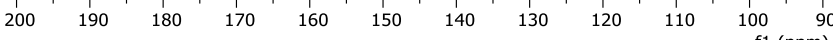

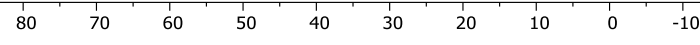


Divergent Synthesis of $\alpha$-Fluorinated Carbonyl and Carboxyl Derivatives by Double Electrophilic Activation of Amides

$\left(\mathrm{CDCl}_{3}, 376 \mathrm{MHz}\right)$

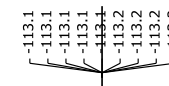

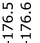

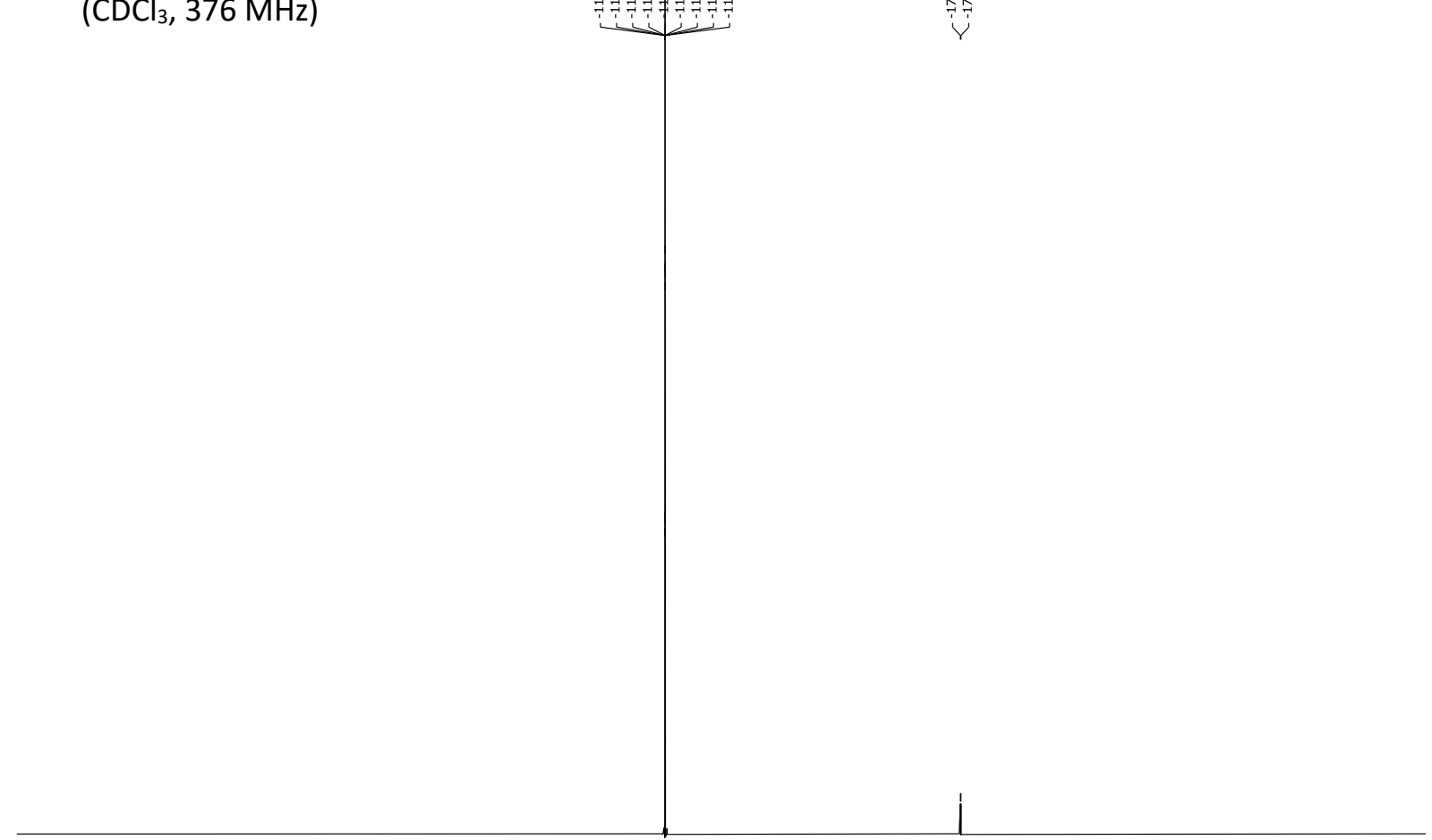

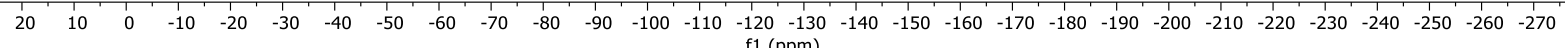
f1 (ppm) 


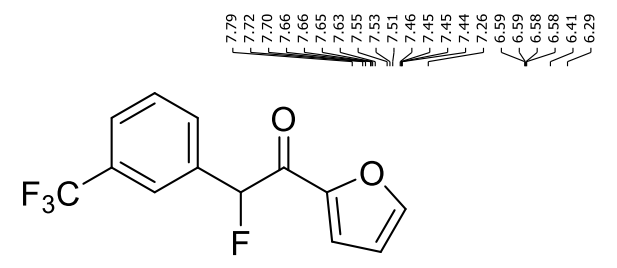

$10 \mathrm{e}$

$\left(\mathrm{CDCl}_{3}, 400 \mathrm{MHz}\right)$

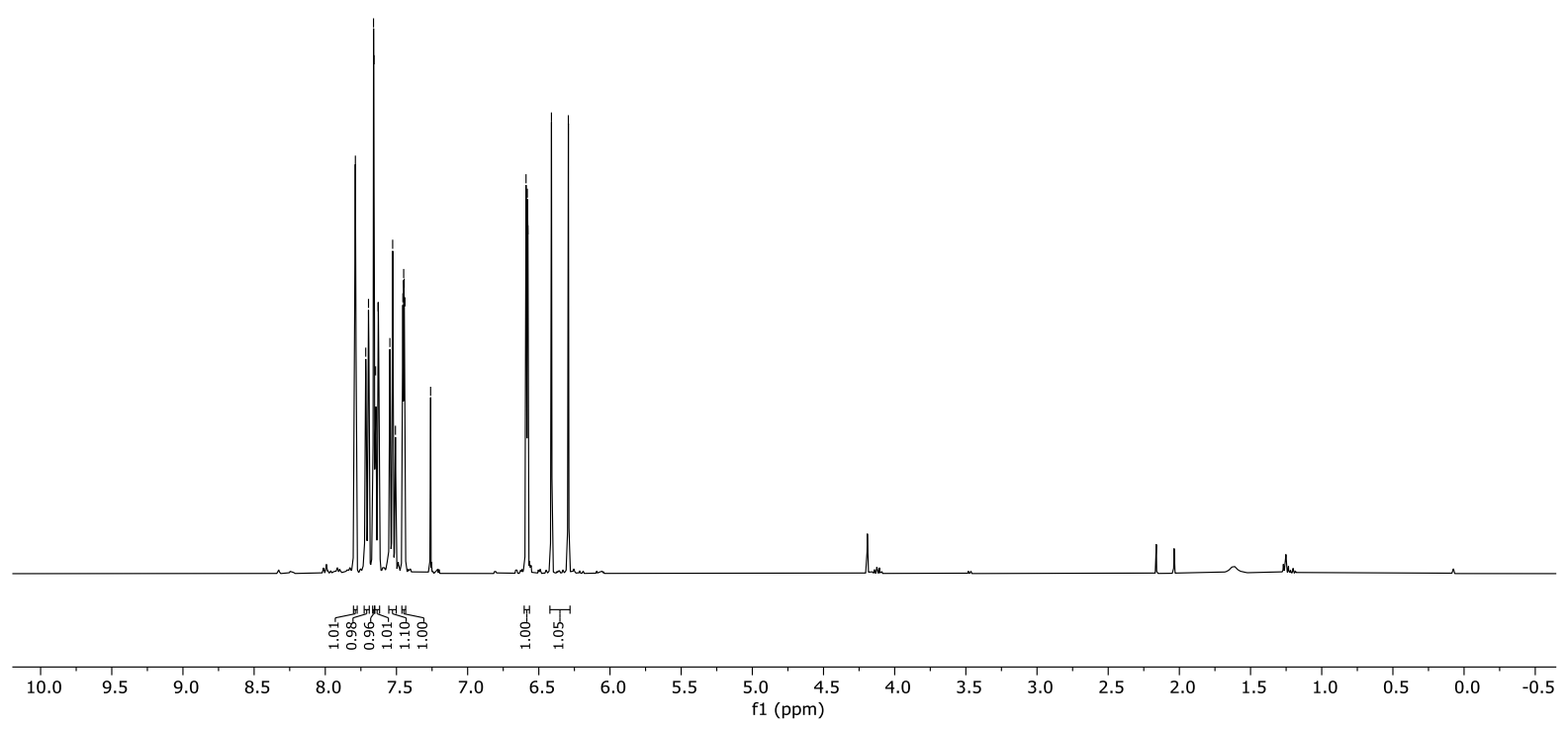

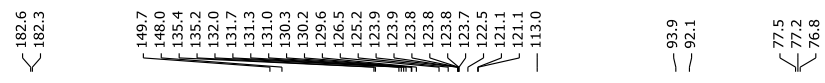

$\left(\mathrm{CDCl}_{3}, 100 \mathrm{MHz}\right)$

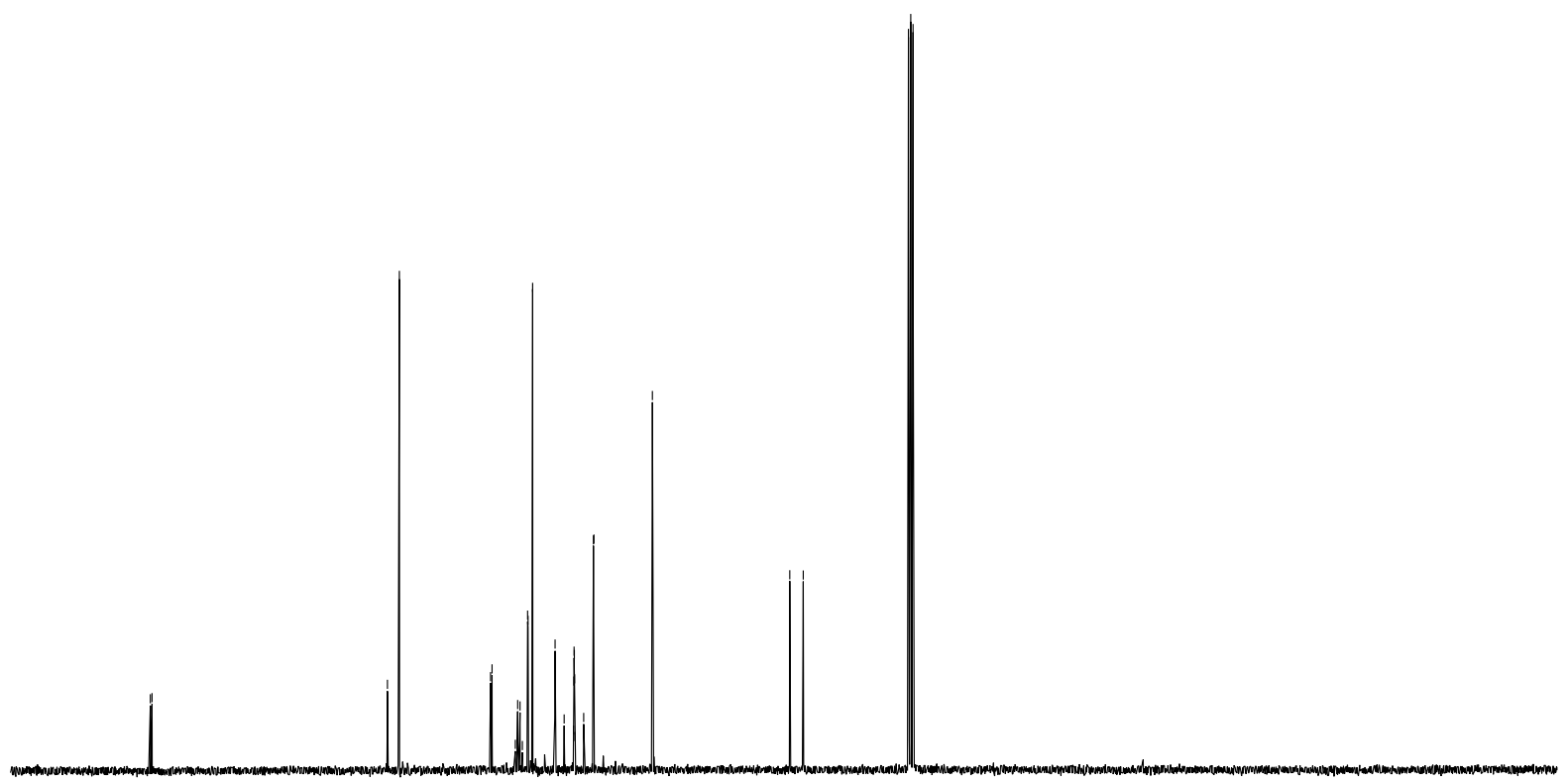

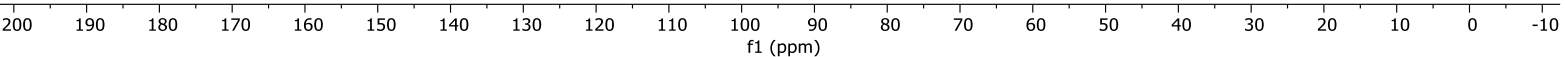


Divergent Synthesis of $\alpha$-Fluorinated Carbonyl and Carboxyl Derivatives by Double Electrophilic Activation of Amides

Internal

standard (fluorobenzene)

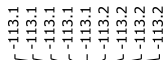

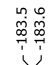

( $\mathrm{CDCl}_{3}, 376 \mathrm{MHz}$ )

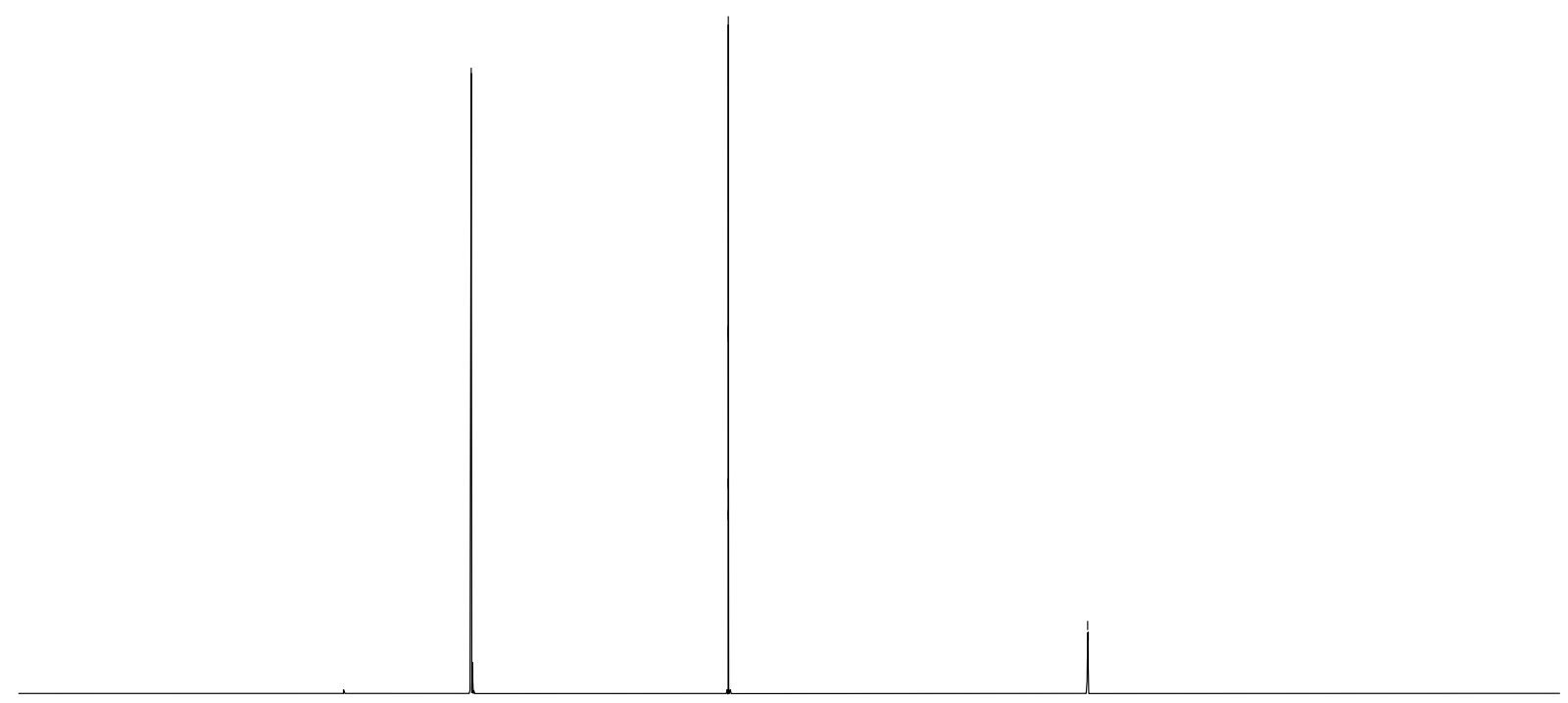

$\begin{array}{llllllllllllllllllllllllllllllllllll}20 & 10 & 0 & -10 & -20 & -30 & -40 & -50 & -60 & -70 & -80 & -90 & -100 & -110 & -120 & -130 & -140 & -150 & -160 & -170 & -180 & -190 & -200 & -210 & -220 & -230 & -240 & -250 & -260 & -270\end{array}$ f1 (ppm) 


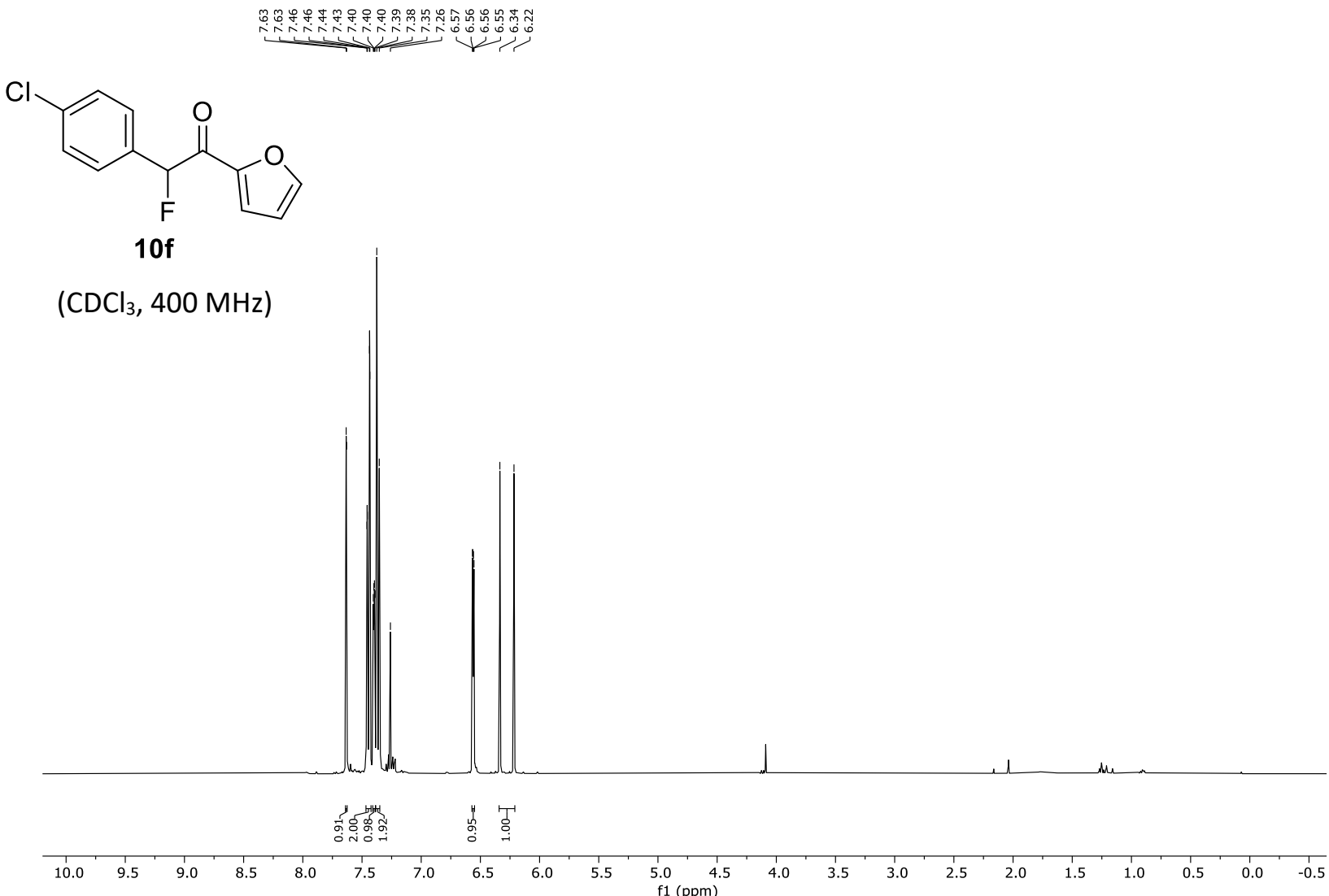

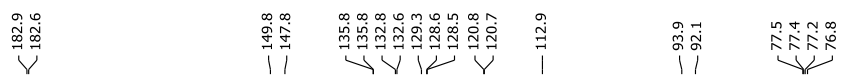

$\left(\mathrm{CDCl}_{3}, 100 \mathrm{MHz}\right)$

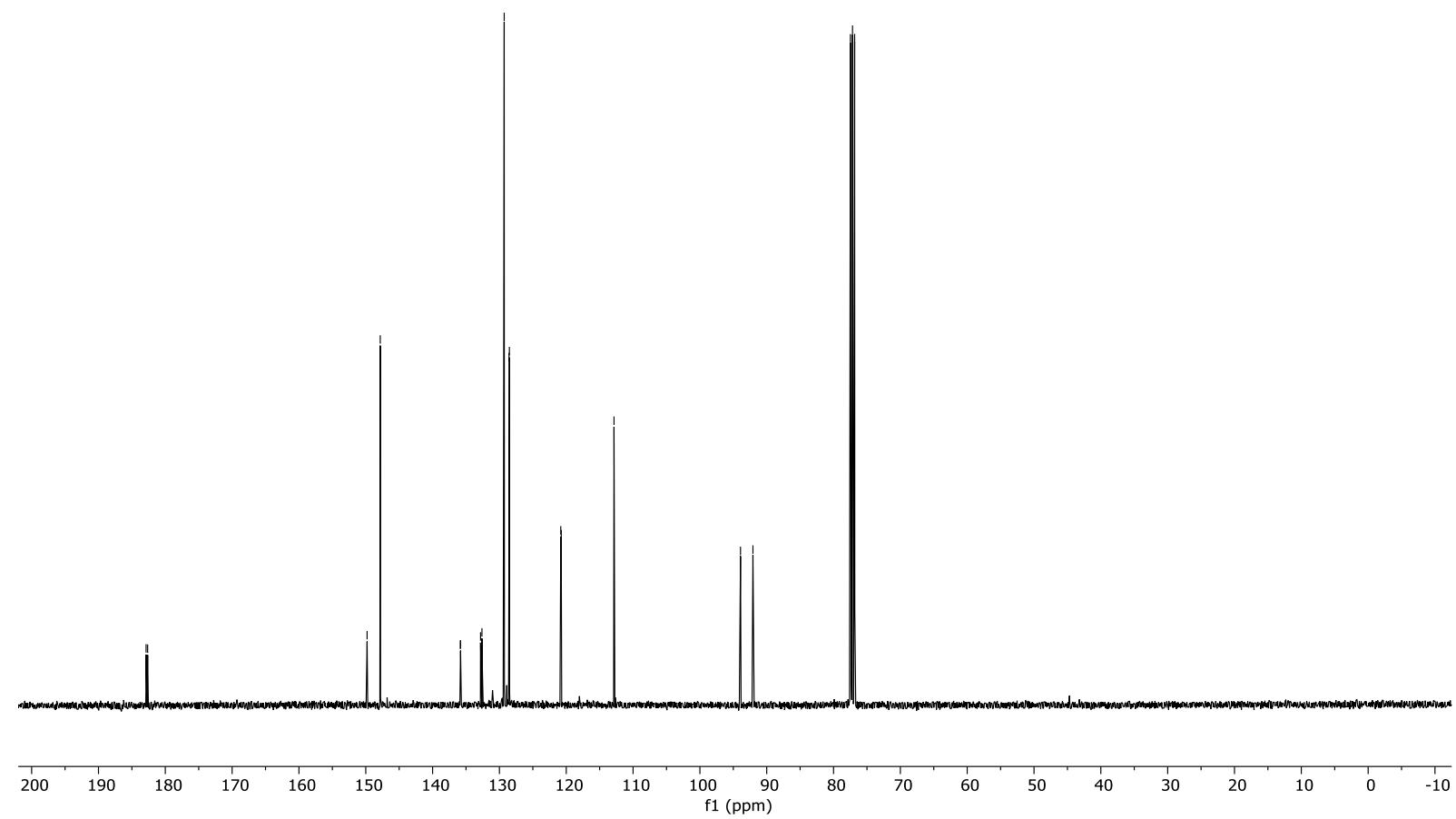


Divergent Synthesis of $\alpha$-Fluorinated Carbonyl and Carboxyl Derivatives by Double Electrophilic Activation of Amides

Internal

standard (fluorobenzene)

$\left(\mathrm{CDCl}_{3}, 376 \mathrm{MHz}\right)$

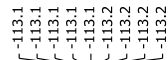

$\underbrace{\substack{\infty \\ i}}_{\substack{\infty \\ i}}$

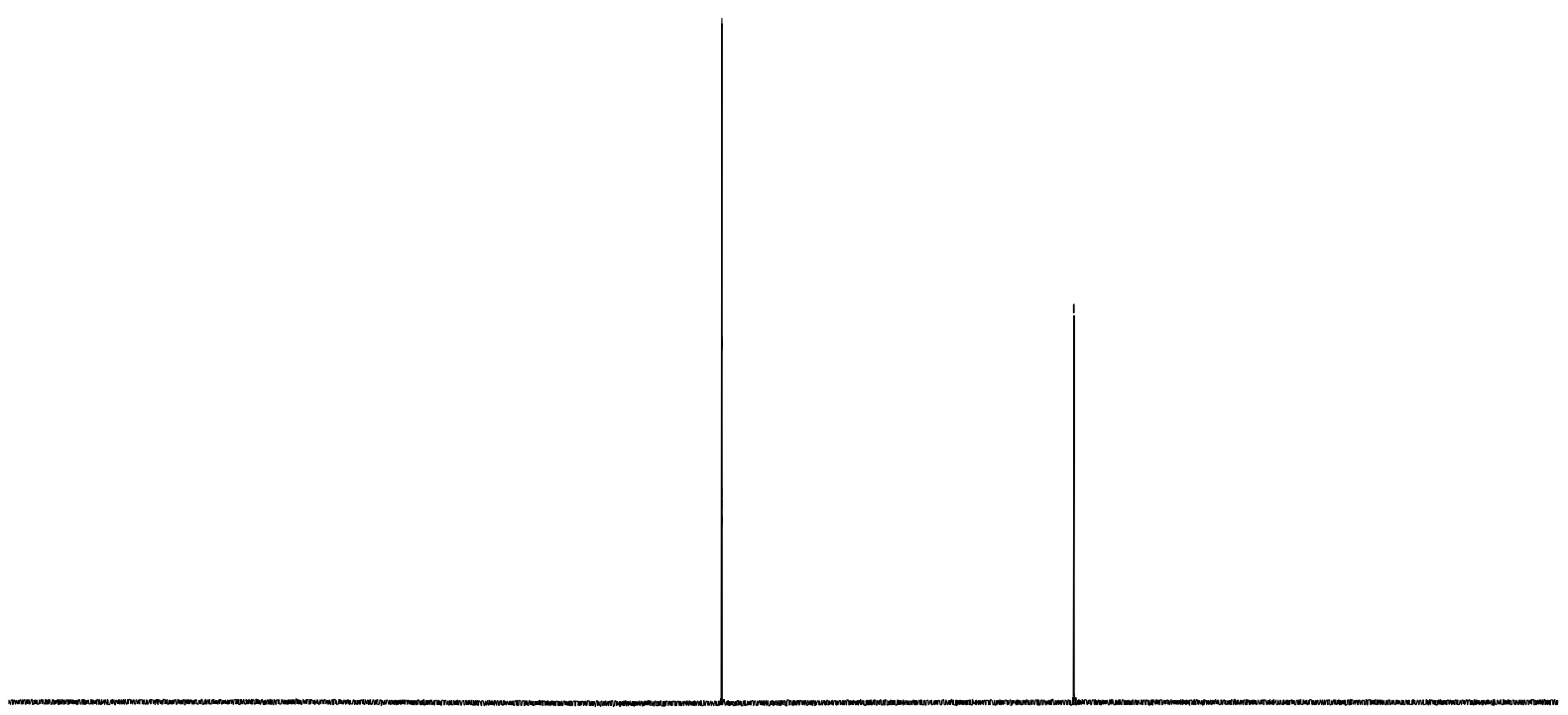

$\begin{array}{llllllllllllllllllllllllllllllllllllllll}20 & 10 & 0 & -10 & -20 & -30 & -40 & -50 & -60 & -70 & -80 & -90 & -100 & -110 & -120 & -130 & -140 & -150 & -160 & -170 & -180 & -190 & -200 & -210 & -220 & -230 & -240 & -250 & -260 & -270\end{array}$ 


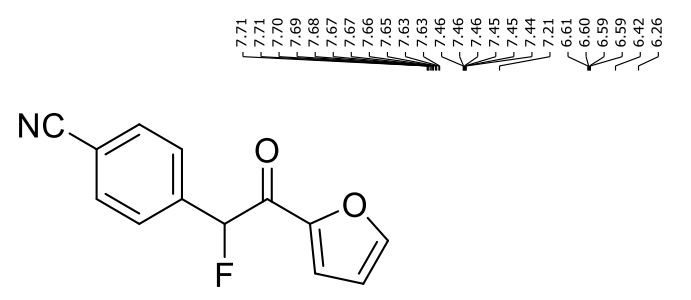

$10 \mathrm{~g}$

$\left(\mathrm{CDCl}_{3}, 400 \mathrm{MHz}\right)$

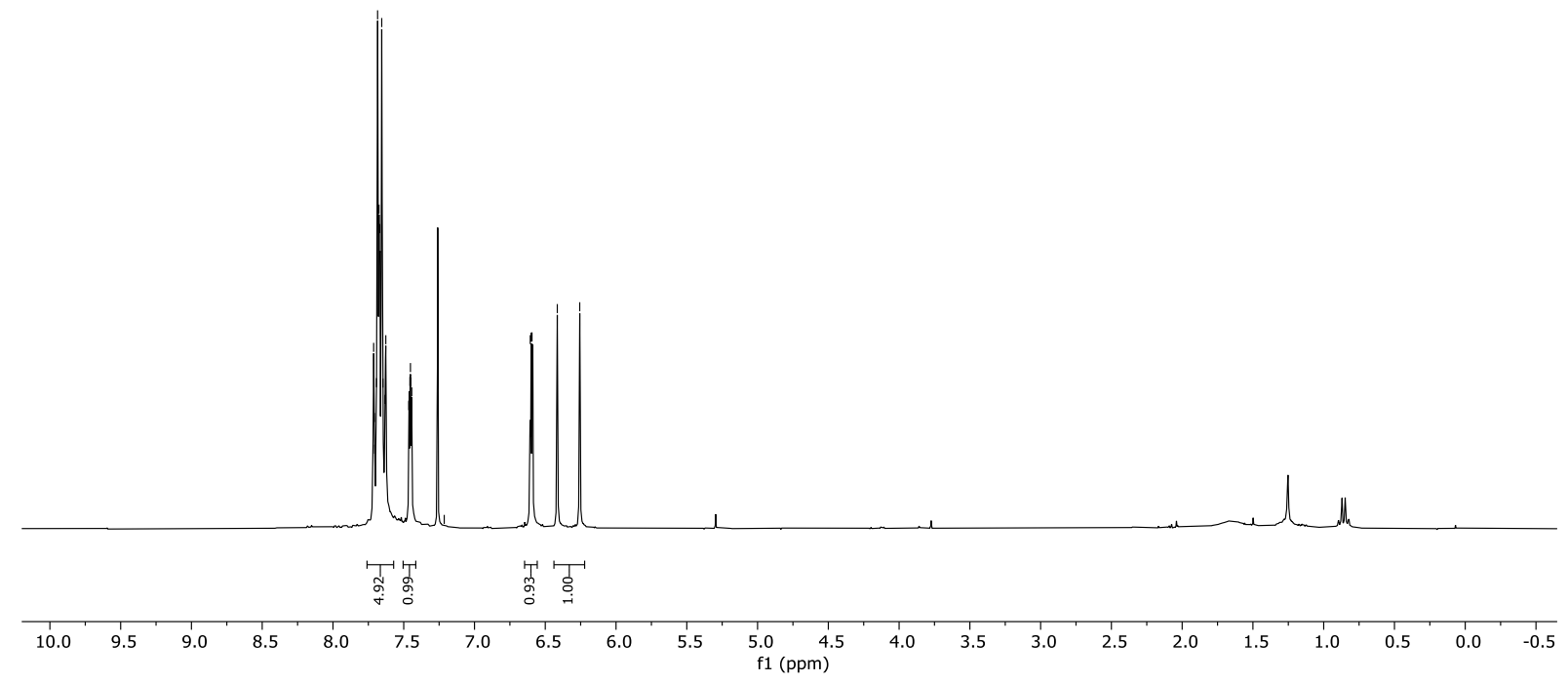

$\left(\mathrm{CDCl}_{3}, 100 \mathrm{MHz}\right)$

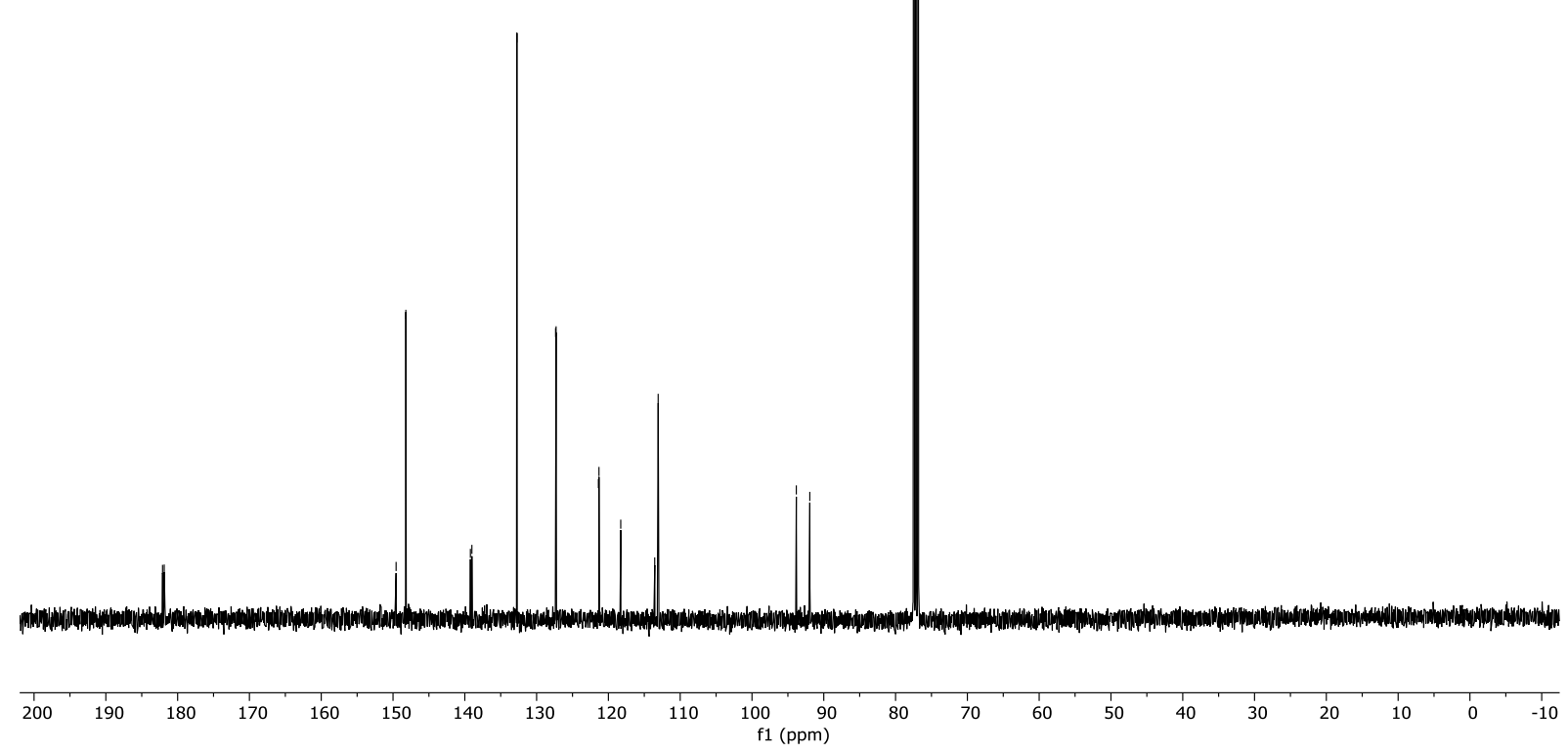


Divergent Synthesis of $\alpha$-Fluorinated Carbonyl and Carboxyl Derivatives by Double Electrophilic Activation of Amides

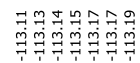

$\left(\mathrm{CDCl}_{3}, 376 \mathrm{MHz}\right)$

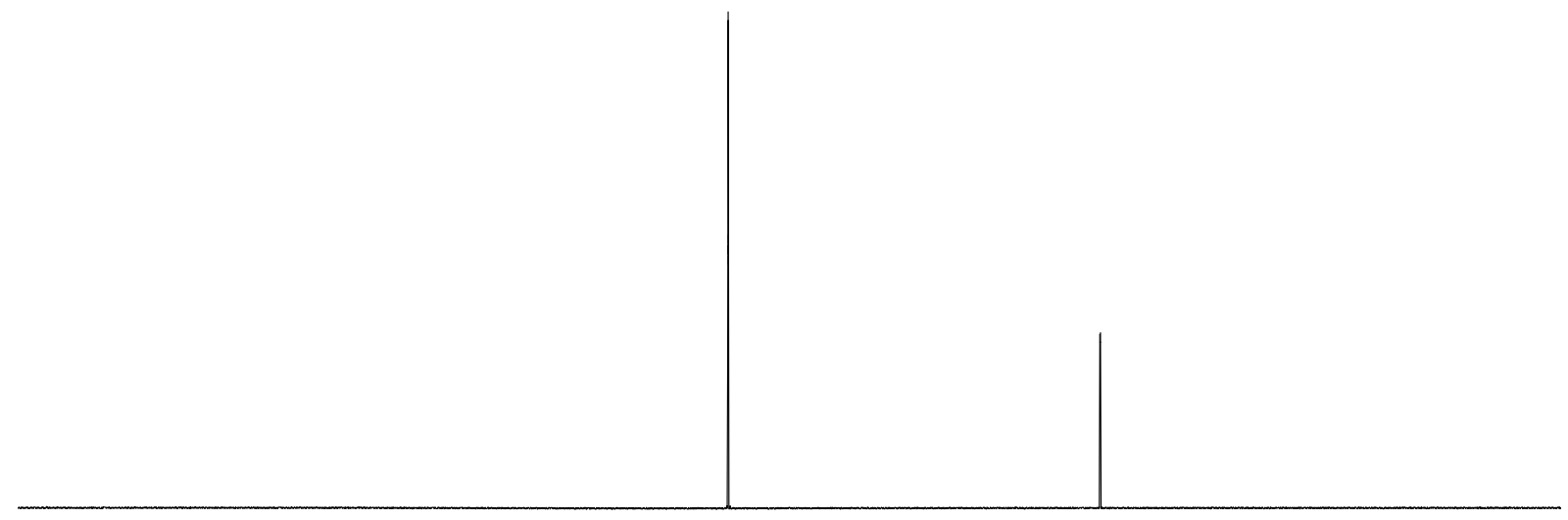

$\begin{array}{llllllllllllllllllllllllllllllllllllllllllllllll}20 & 10 & 0 & -10 & -20 & -30 & -40 & -50 & -60 & -70 & -80 & -90 & -100 & -110 & -120 & -130 & -140 & -150 & -160 & -170 & -180 & -190 & -200 & -210 & -220 & -230 & -240 & -250 & -260 & -270\end{array}$ 


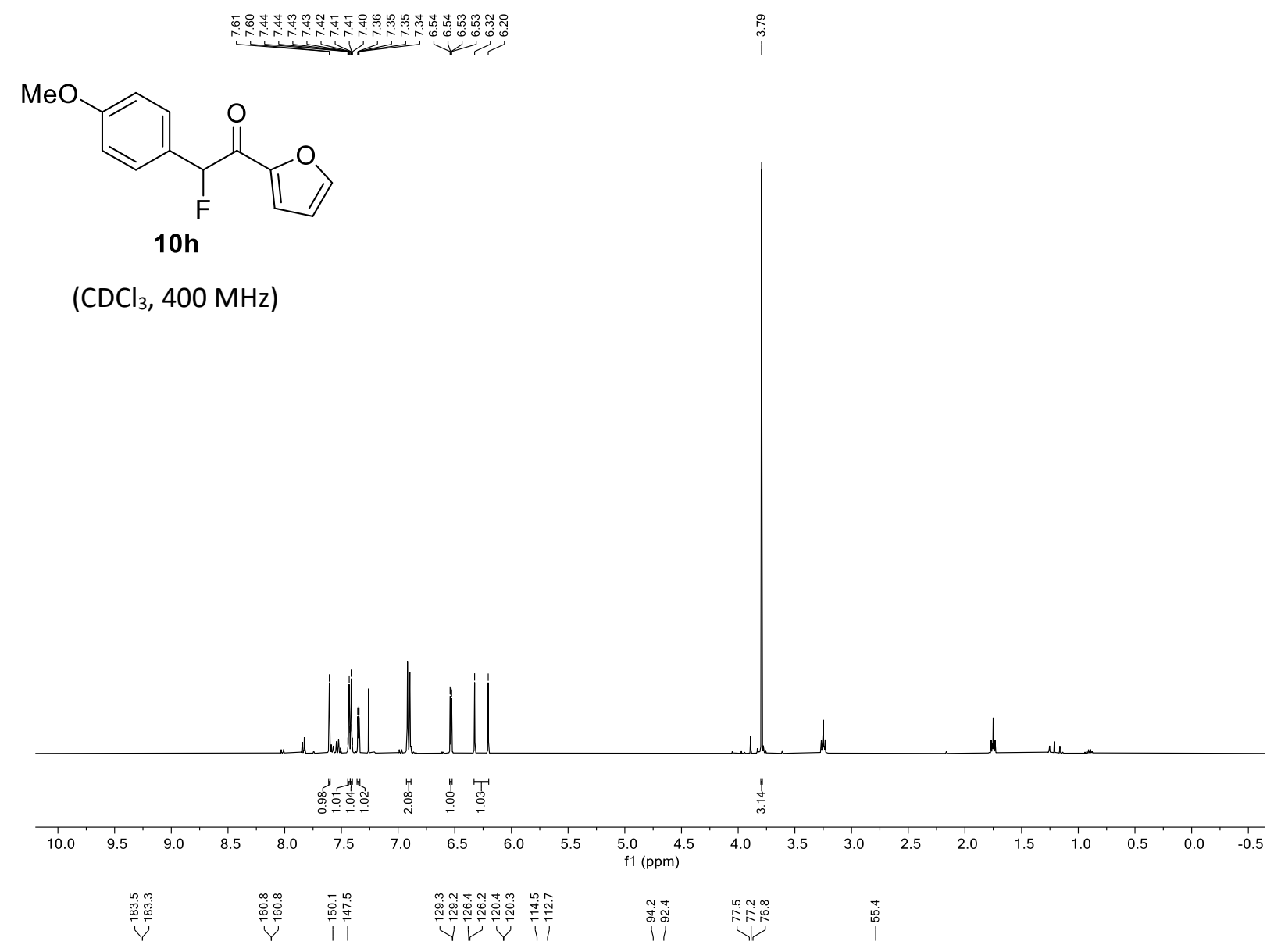

$\left(\mathrm{CDCl}_{3}, 100 \mathrm{MHz}\right)$

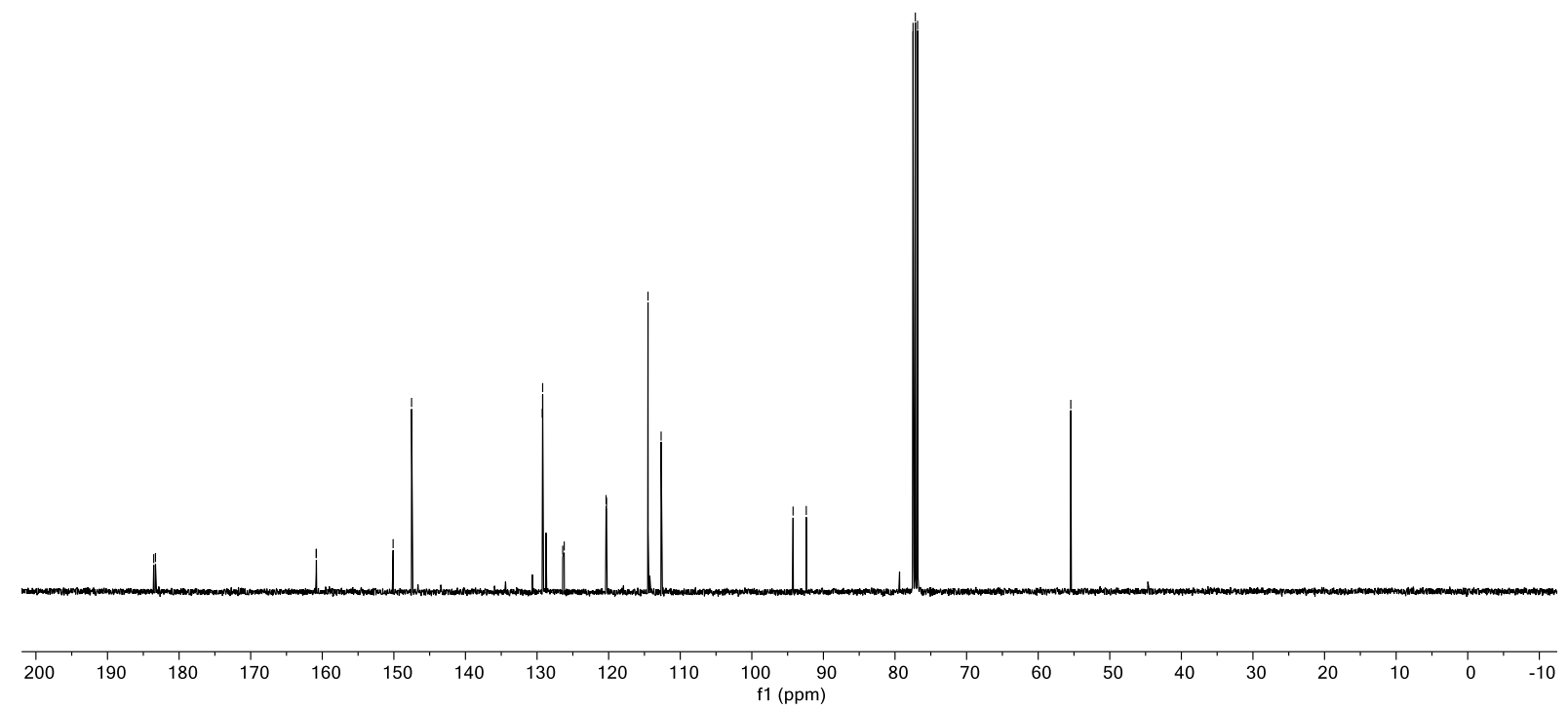


Divergent Synthesis of $\alpha$-Fluorinated Carbonyl and Carboxyl Derivatives by Double Electrophilic Activation of Amides

Internal

standard (fluorobenzene)

$\left(\mathrm{CDCl}_{3}, 376 \mathrm{MHz}\right)$

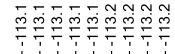

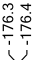

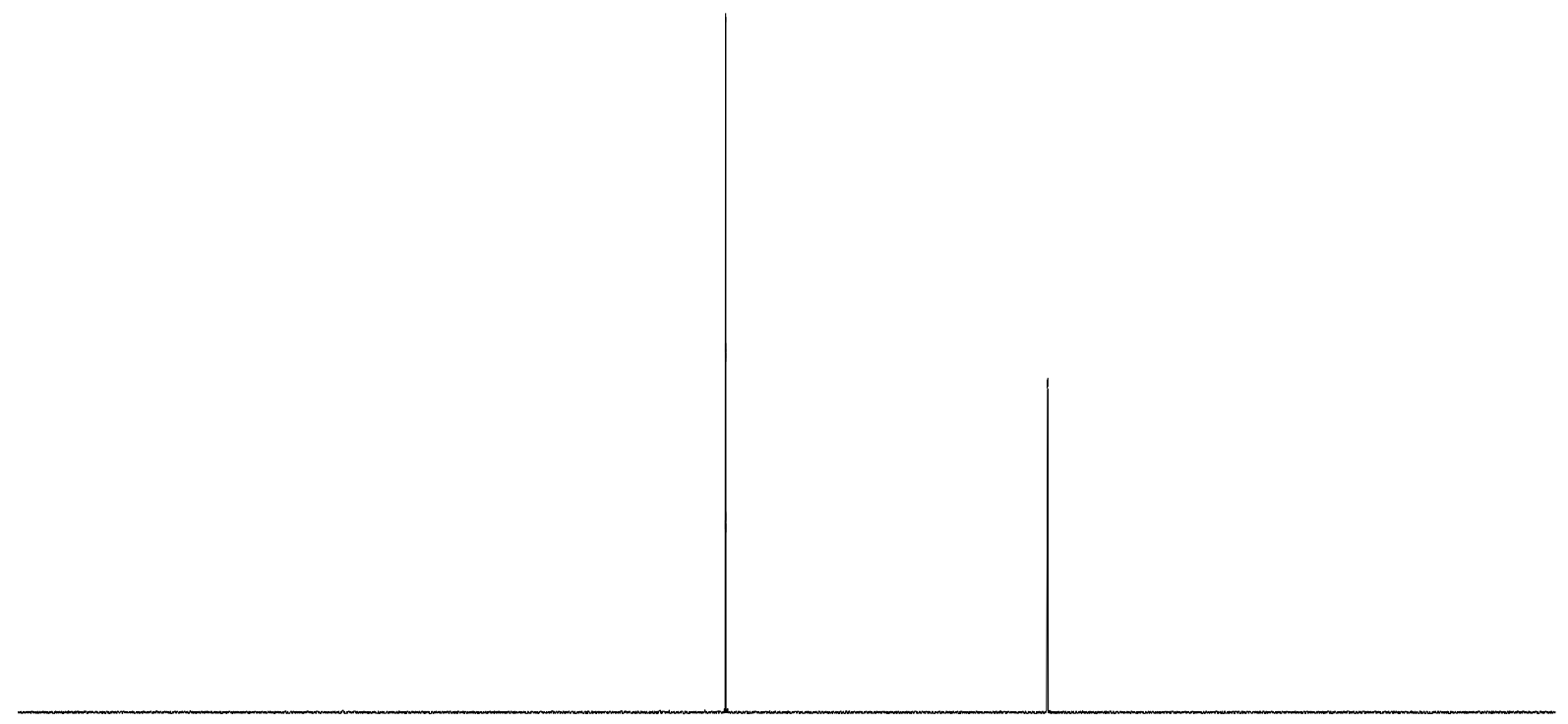

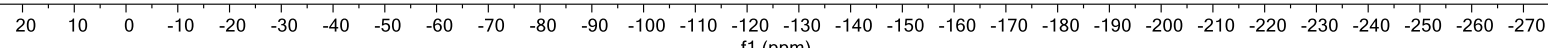
$\mathrm{f1}$ (ppm) 


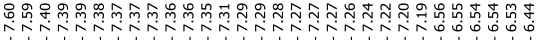<smiles>Cc1ccccc1C(F)C(=O)c1ccco1</smiles>

10j

$\left(\mathrm{CDCl}_{3}, 400 \mathrm{MHz}\right)$
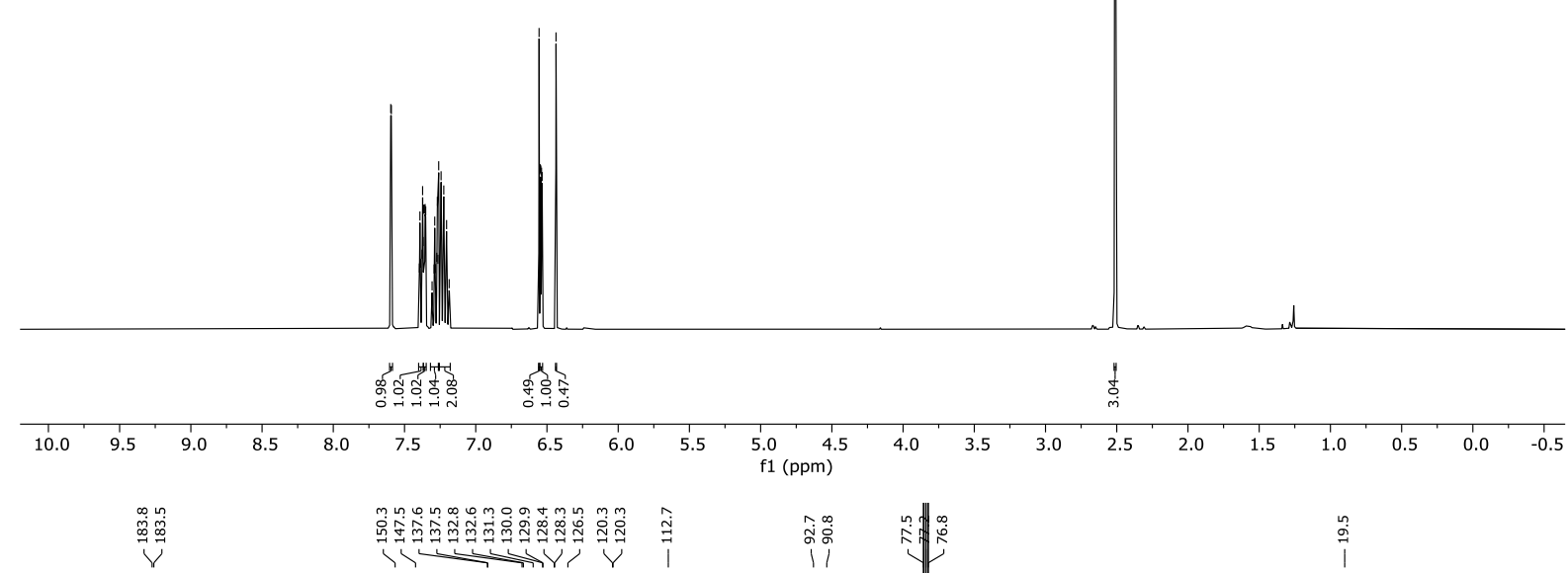

în.

$\stackrel{\text { L }}{\text { I }}$

$\left(\mathrm{CDCl}_{3}, 100 \mathrm{MHz}\right)$

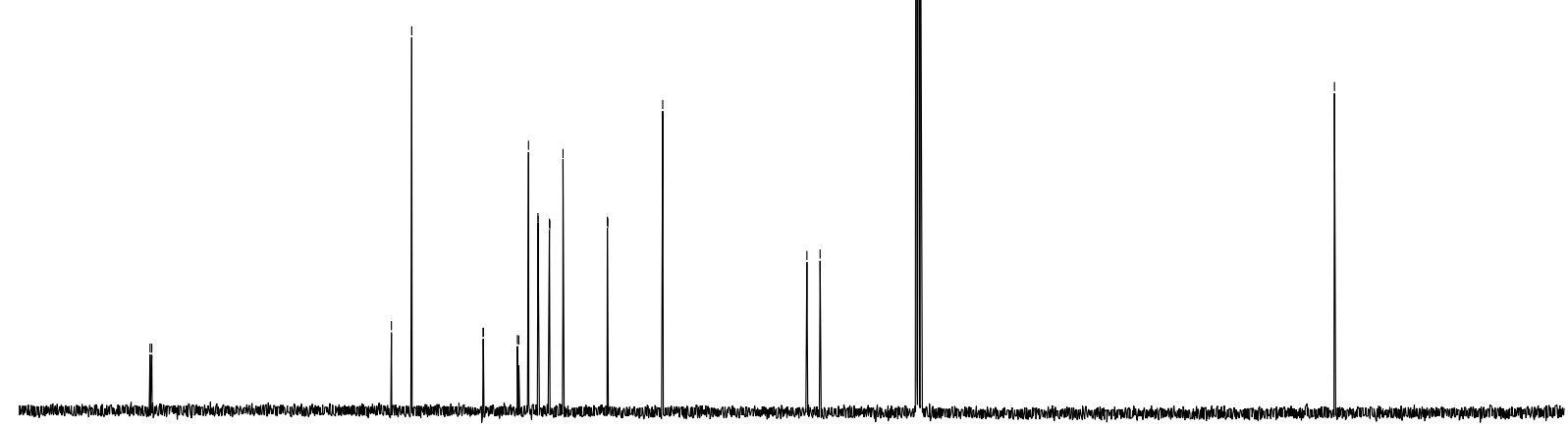

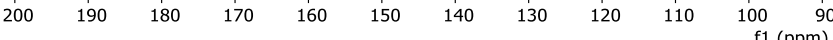


Divergent Synthesis of $\alpha$-Fluorinated Carbonyl and Carboxyl Derivatives by Double Electrophilic Activation of Amides

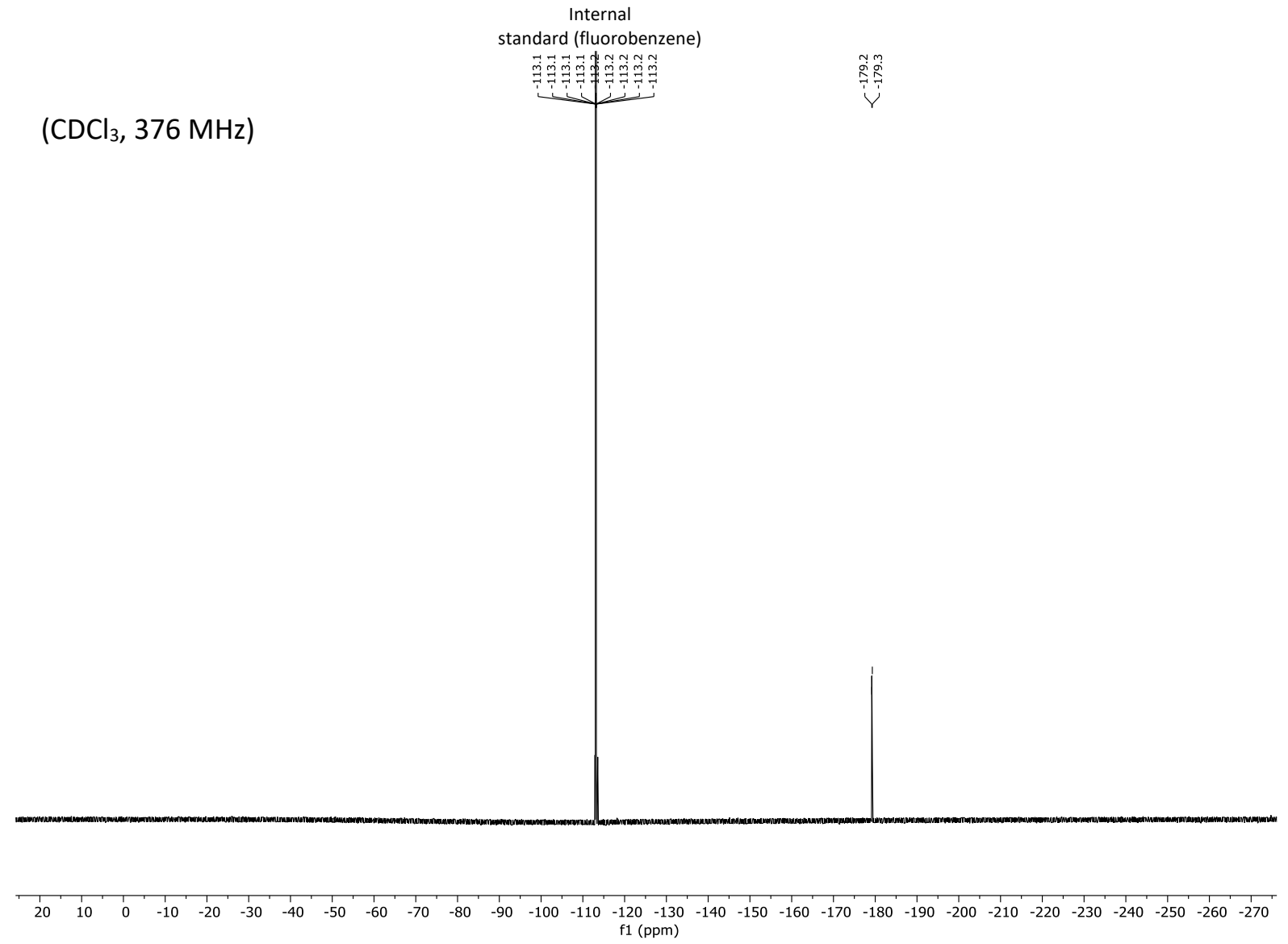




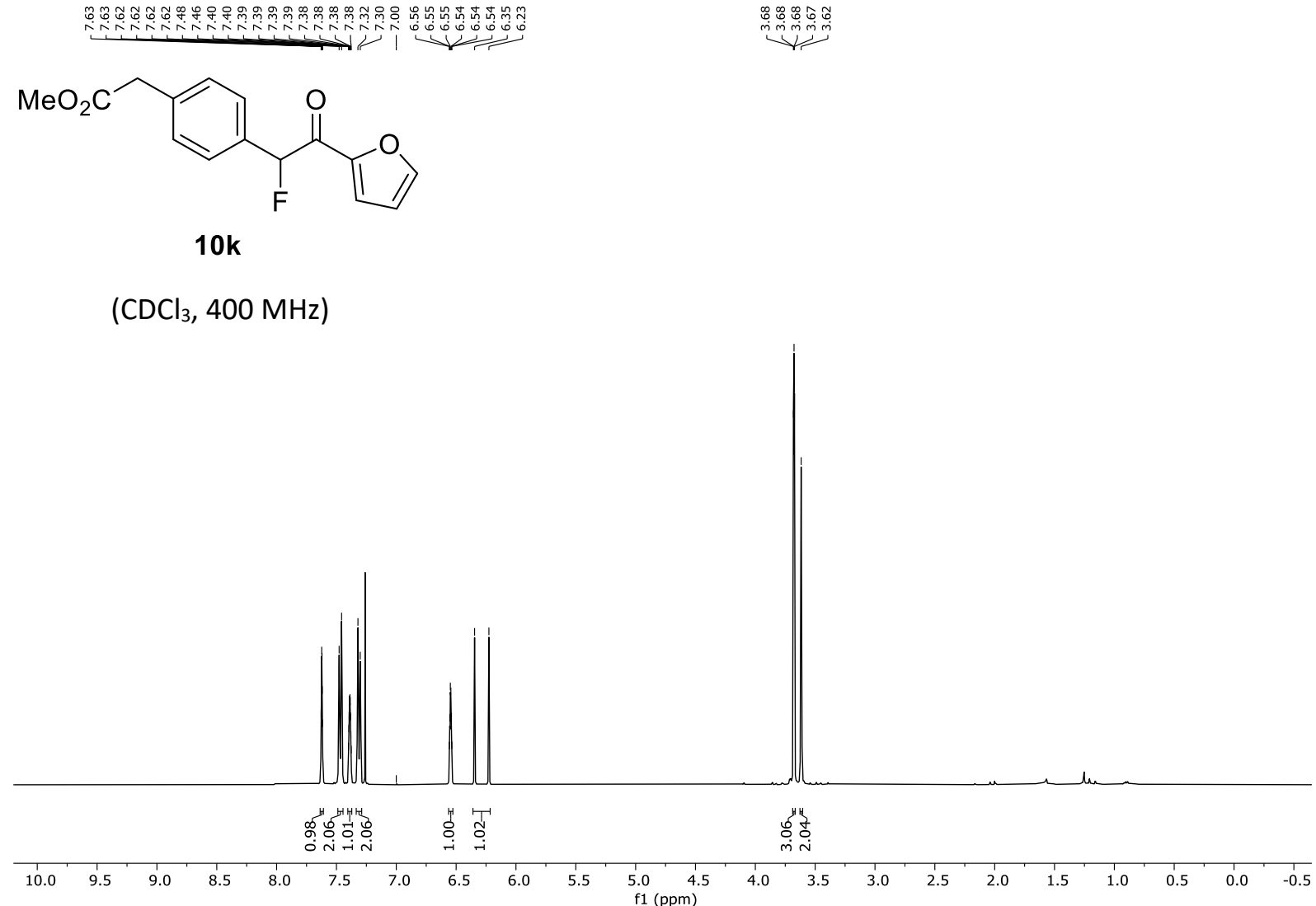

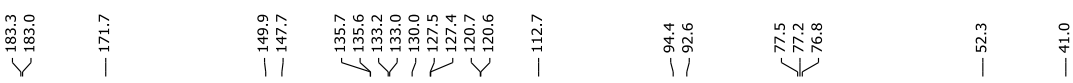

$\left(\mathrm{CDCl}_{3}, 100 \mathrm{MHz}\right)$

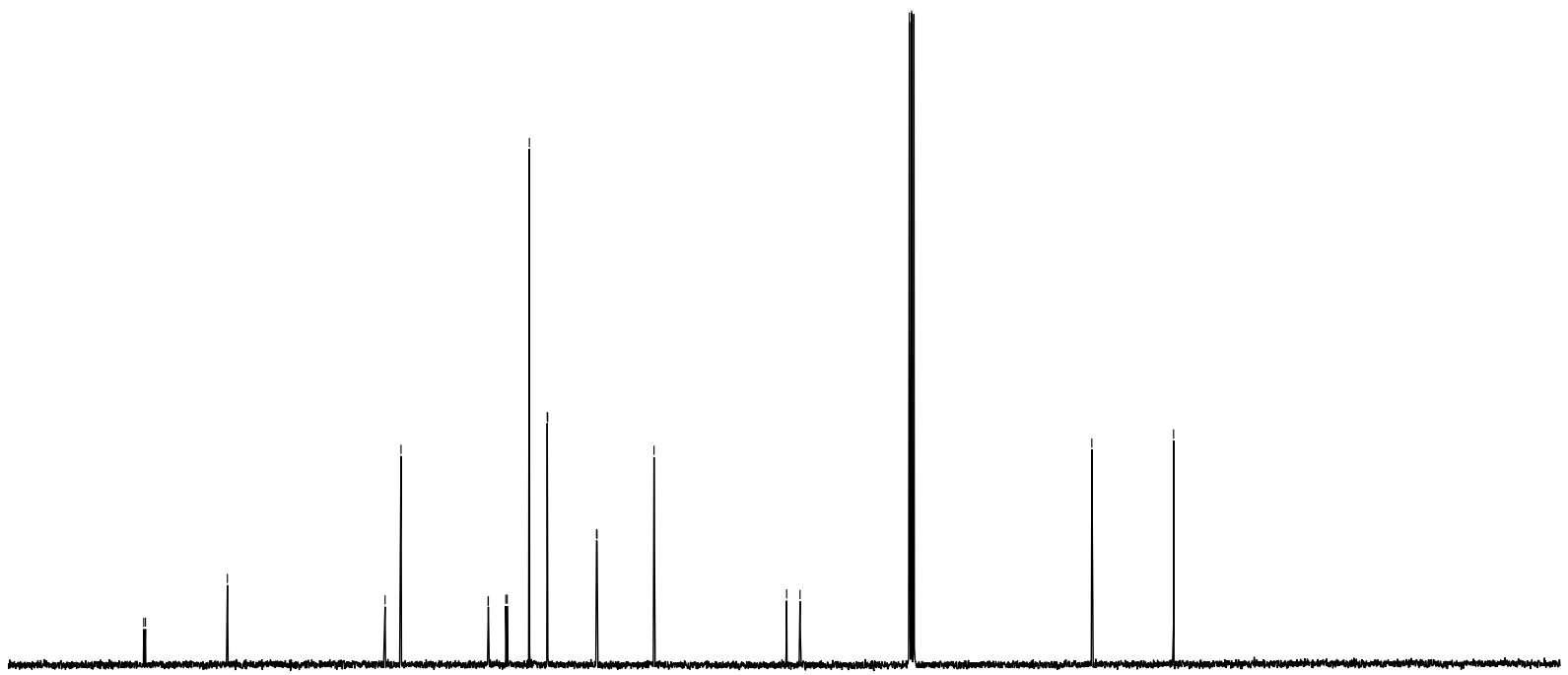

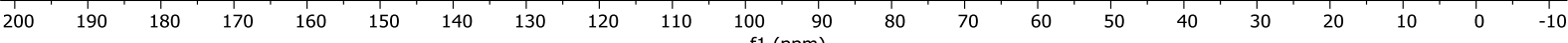


Divergent Synthesis of $\alpha$-Fluorinated Carbonyl and Carboxyl Derivatives by Double Electrophilic Activation of Amides

\section{Internal \\ standard (fluorobenzene)}

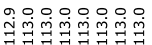

$\left(\mathrm{CDCl}_{3}, 376 \mathrm{MHz}\right)$

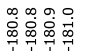

$\checkmark$

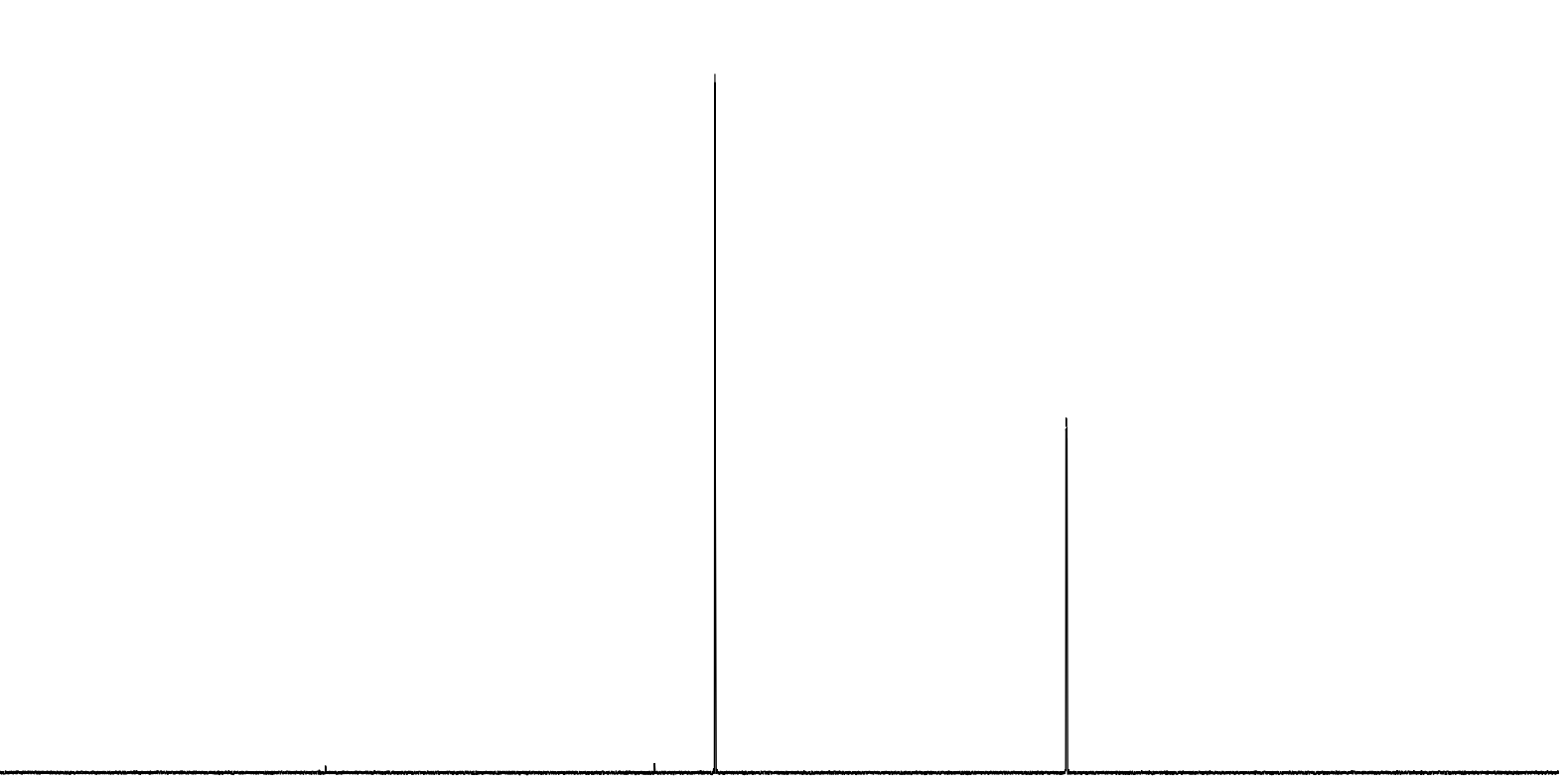

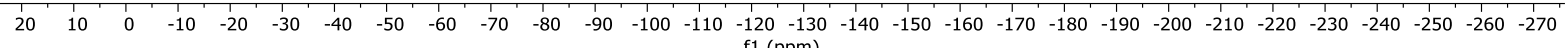
f1 (ppm) 

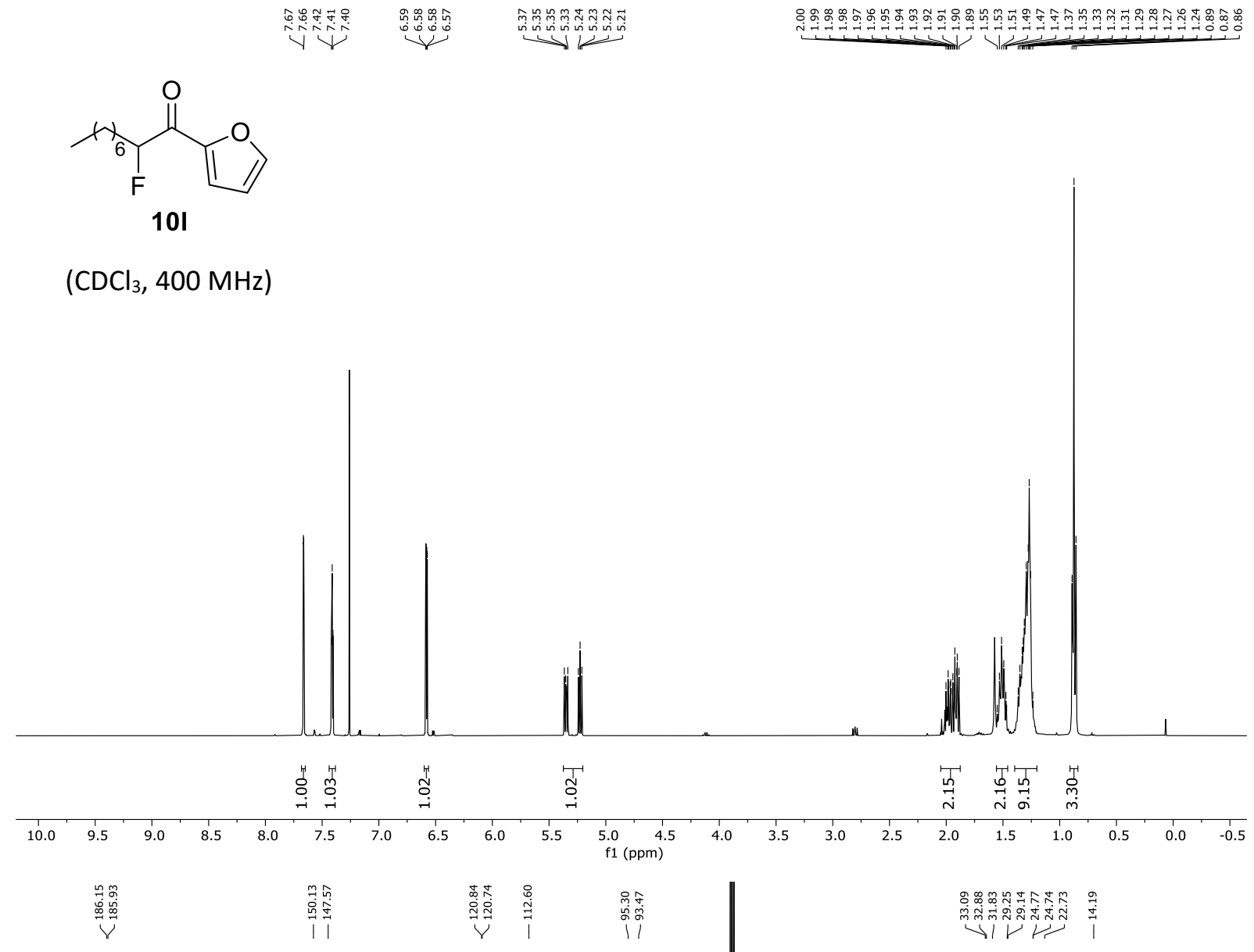

$\left(\mathrm{CDCl}_{3}, 100 \mathrm{MHz}\right)$
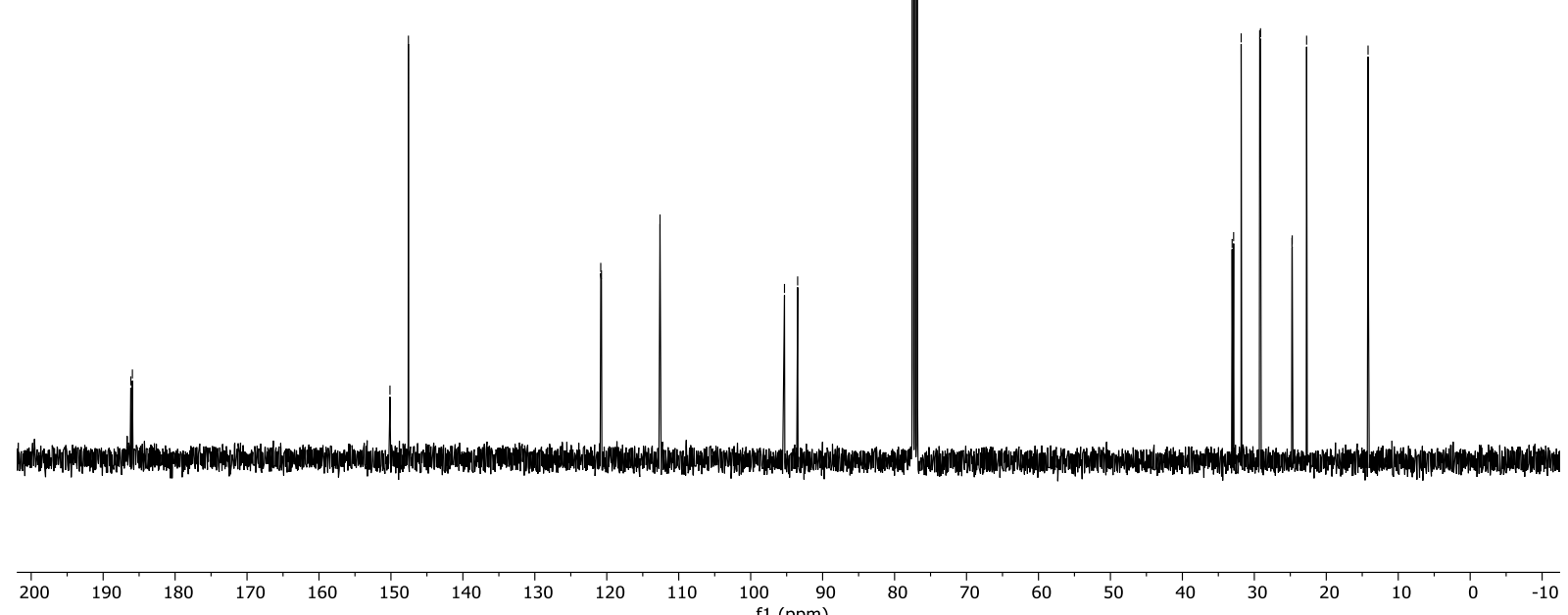
Divergent Synthesis of $\alpha$-Fluorinated Carbonyl and Carboxyl Derivatives by Double Electrophilic Activation of Amides

Internal

standard (fluorobenzene)

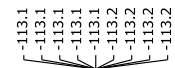

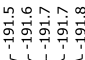

$\left(\mathrm{CDCl}_{3}, 376 \mathrm{MHz}\right)$

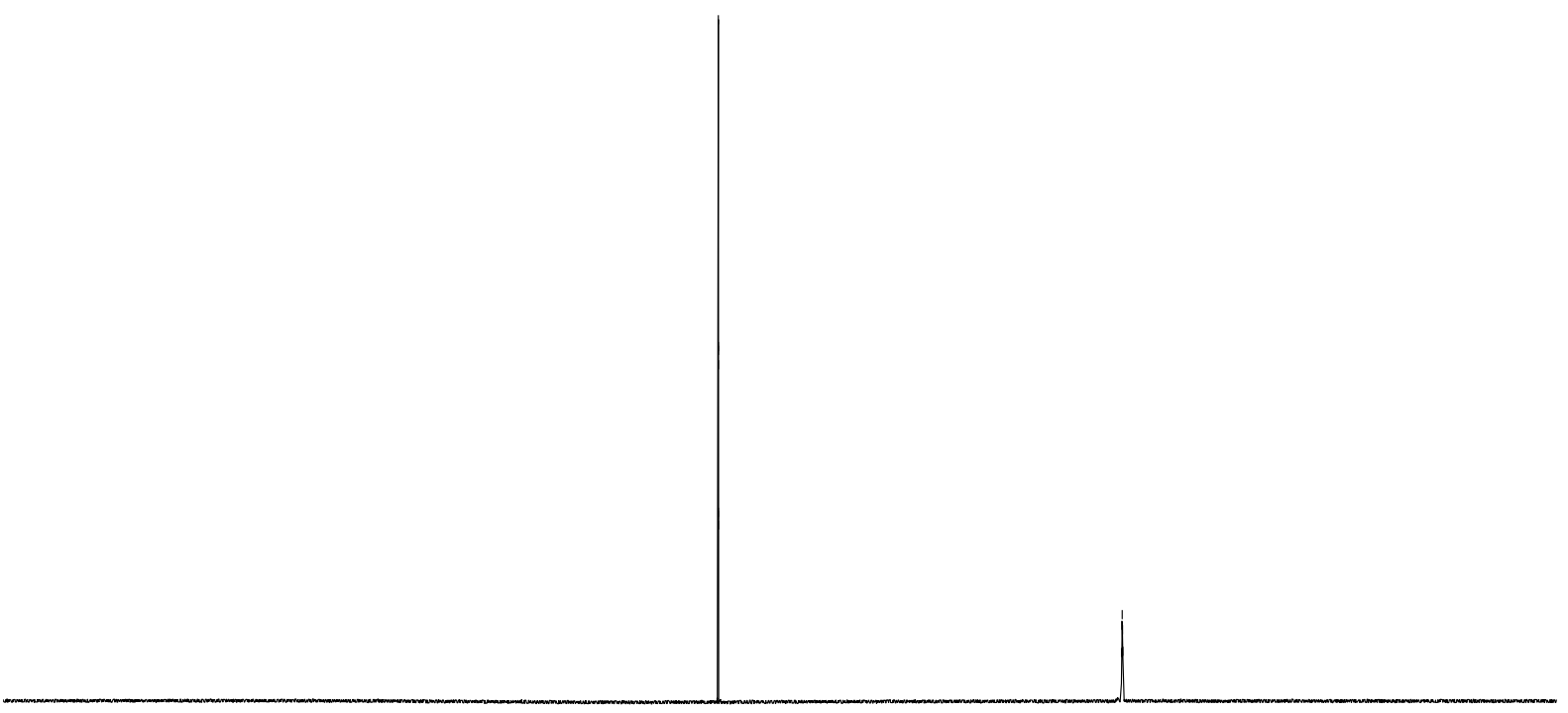

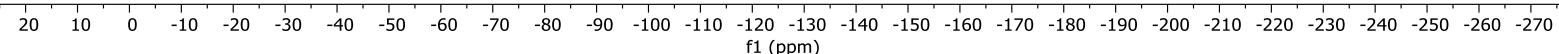




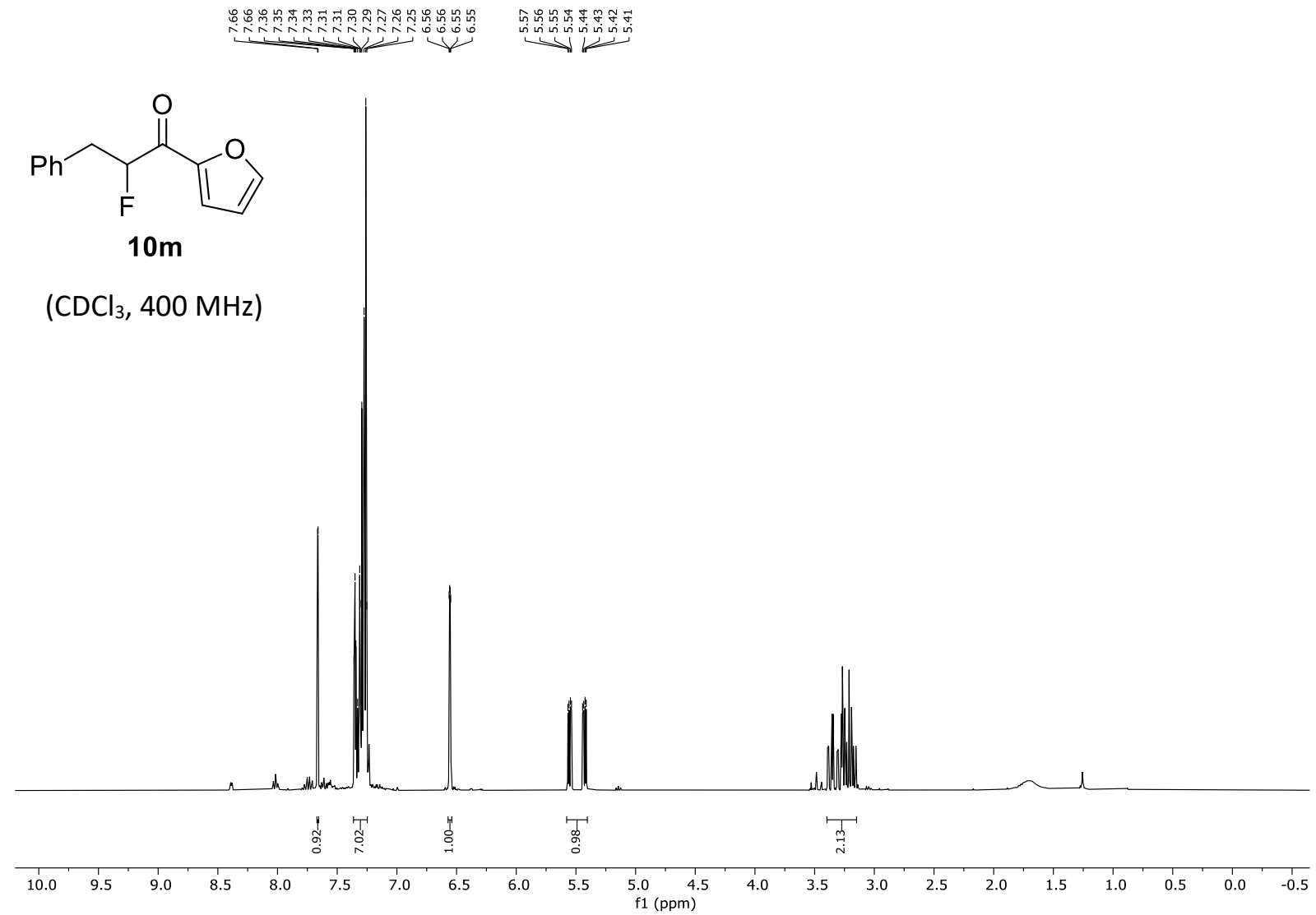

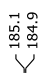

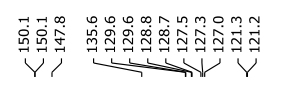

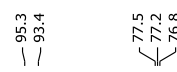

ฟNo.

$\left(\mathrm{CDCl}_{3}, 100 \mathrm{MHz}\right)$

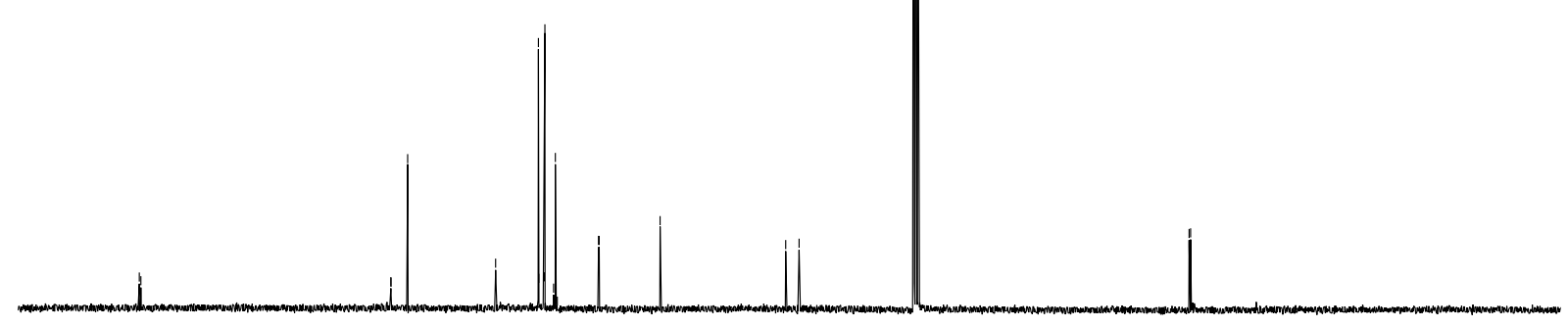

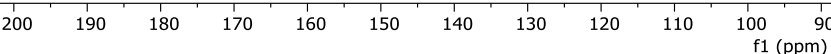


Divergent Synthesis of $\alpha$-Fluorinated Carbonyl and Carboxyl Derivatives by Double Electrophilic Activation of Amides

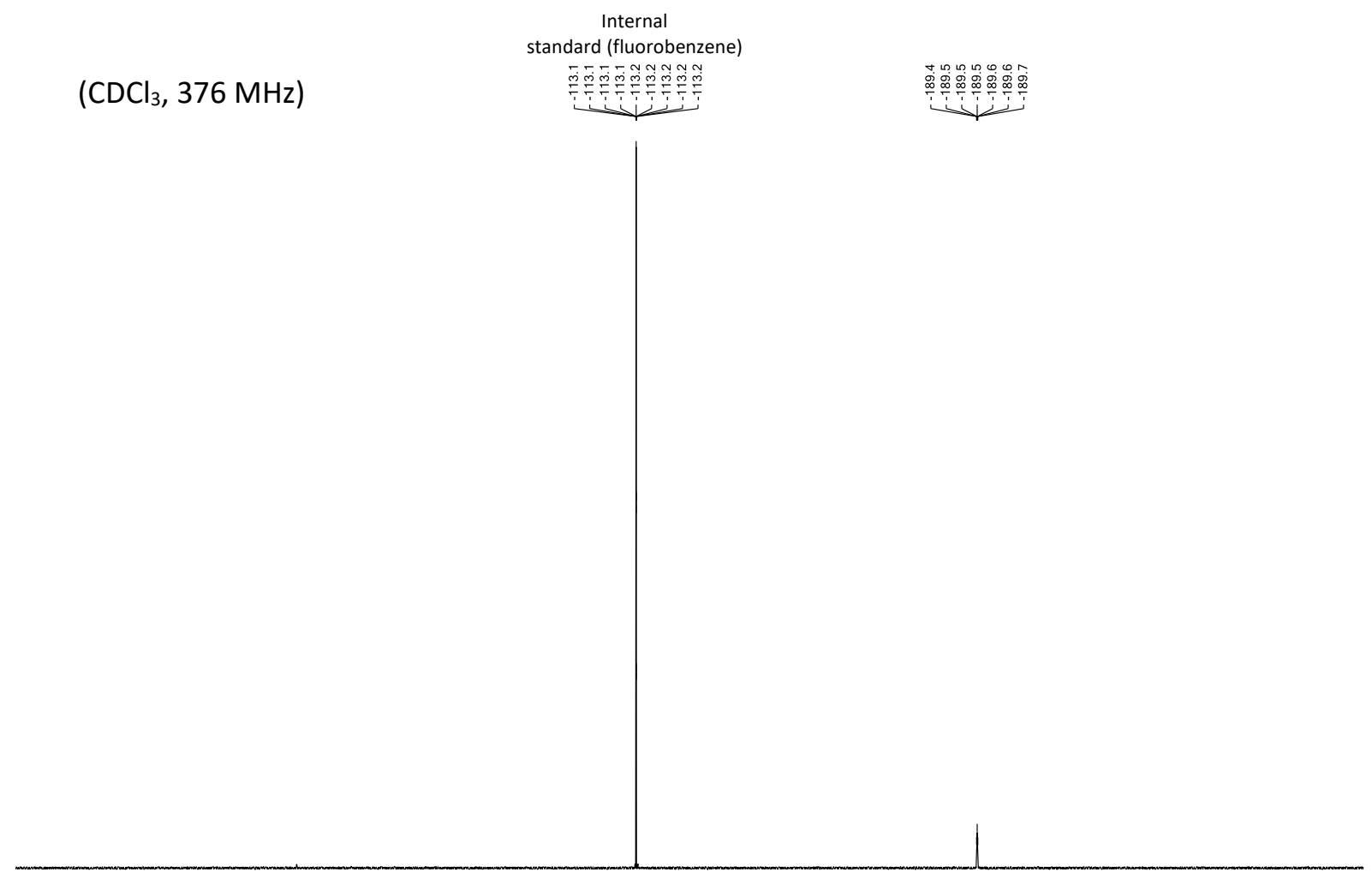

$\begin{array}{lllllllllllllllllllllllllllllllllllllll}20 & 10 & 0 & -10 & -20 & -30 & -40 & -50 & -60 & -70 & -80 & -90 & -100 & -110 & -120 & -130 & -140 & -150 & -160 & -170 & -180 & -190 & -200 & -210 & -220 & -230 & -240 & -250 & -260 & -270\end{array}$ 


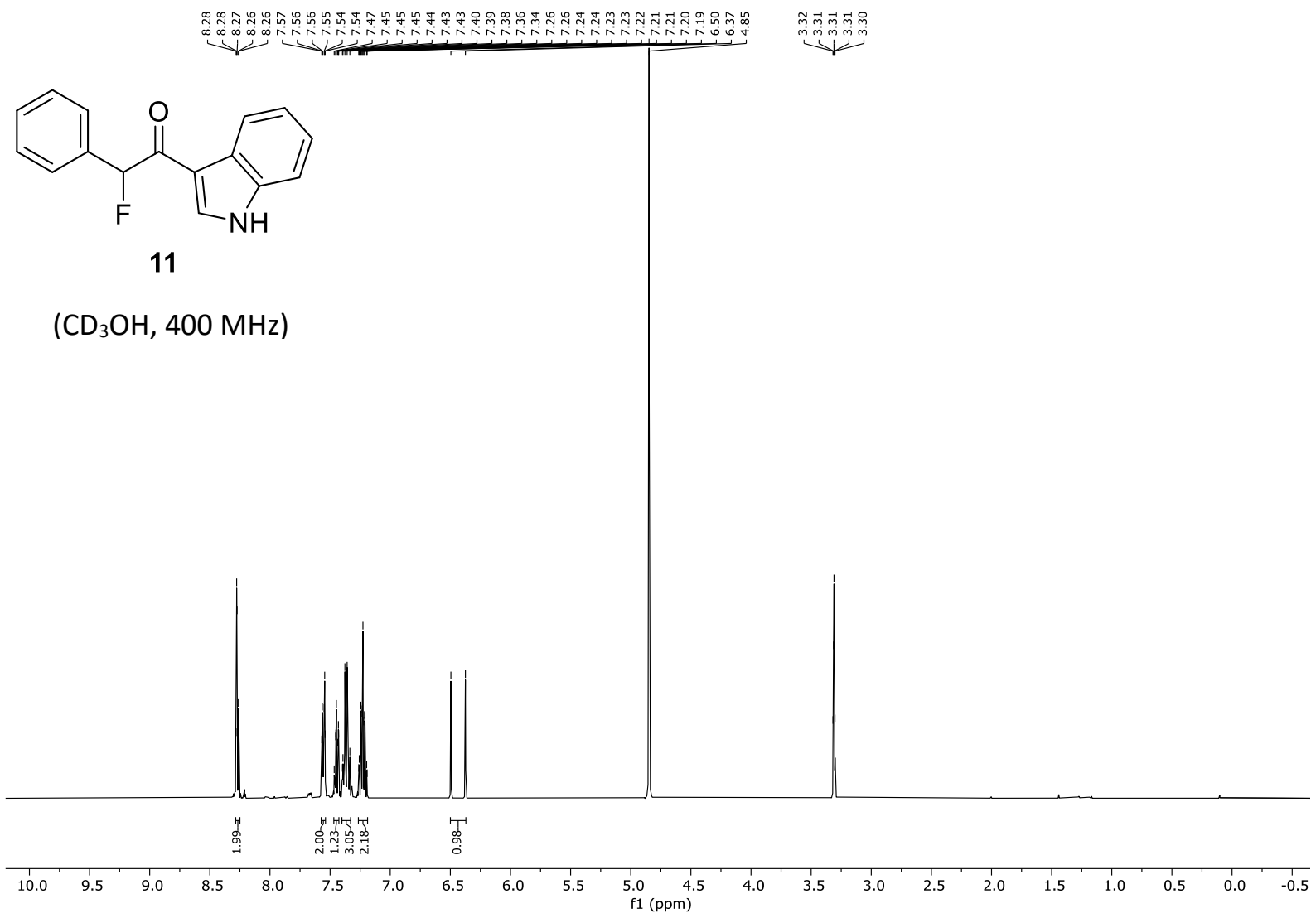

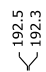

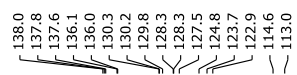

管暴

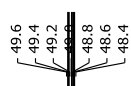

$\left(\mathrm{CD}_{3} \mathrm{OH}, 100 \mathrm{MHz}\right)$

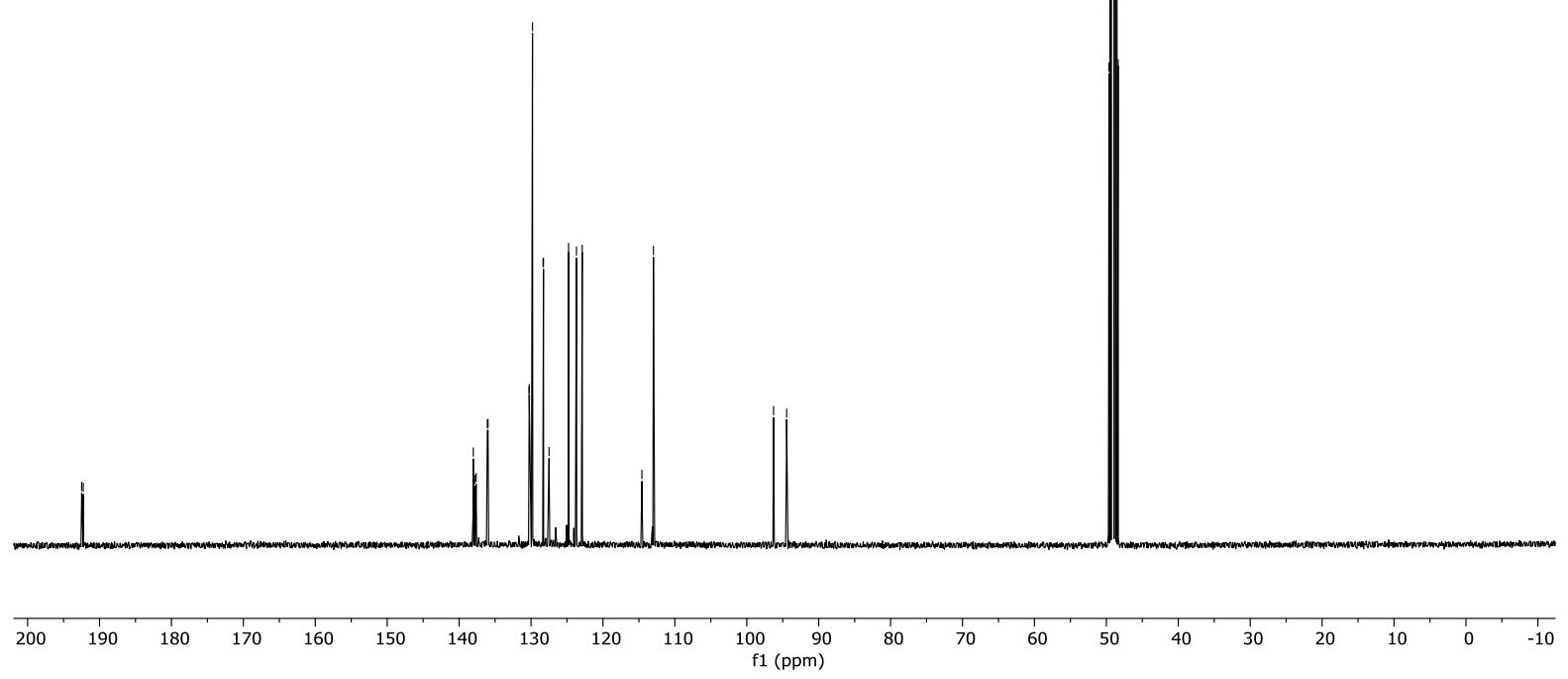


Divergent Synthesis of $\alpha$-Fluorinated Carbonyl and Carboxyl Derivatives by Double Electrophilic Activation of Amides

Internal
standard (fluorobenzene)

$\left(\mathrm{CD}_{3} \mathrm{OH}, 376 \mathrm{MHz}\right)$

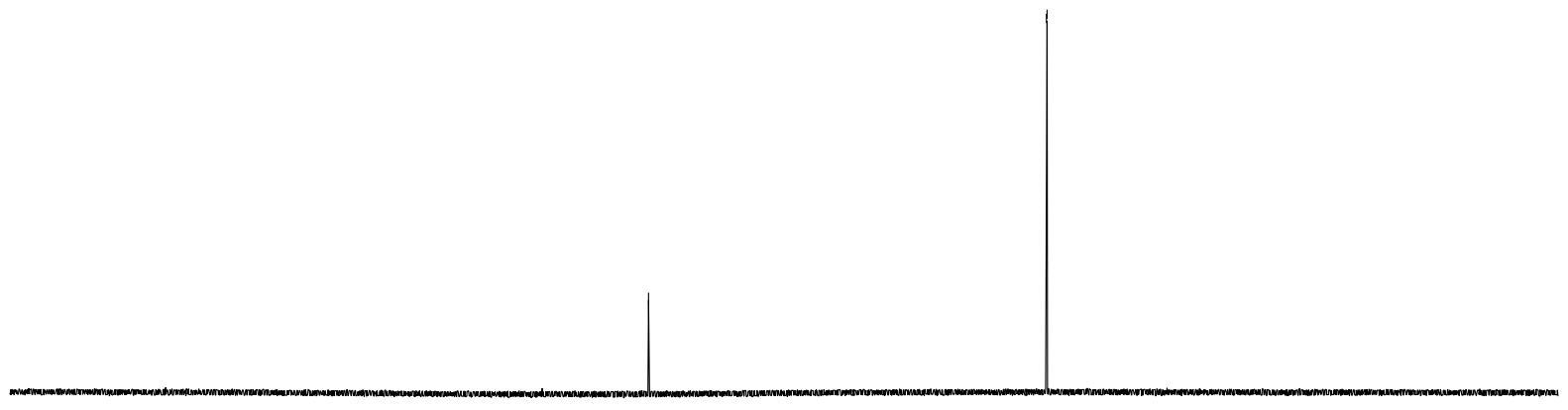

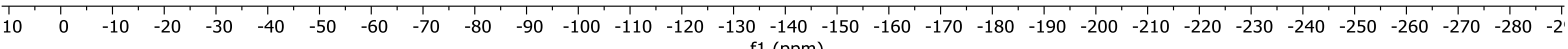
f1 (ppm) 


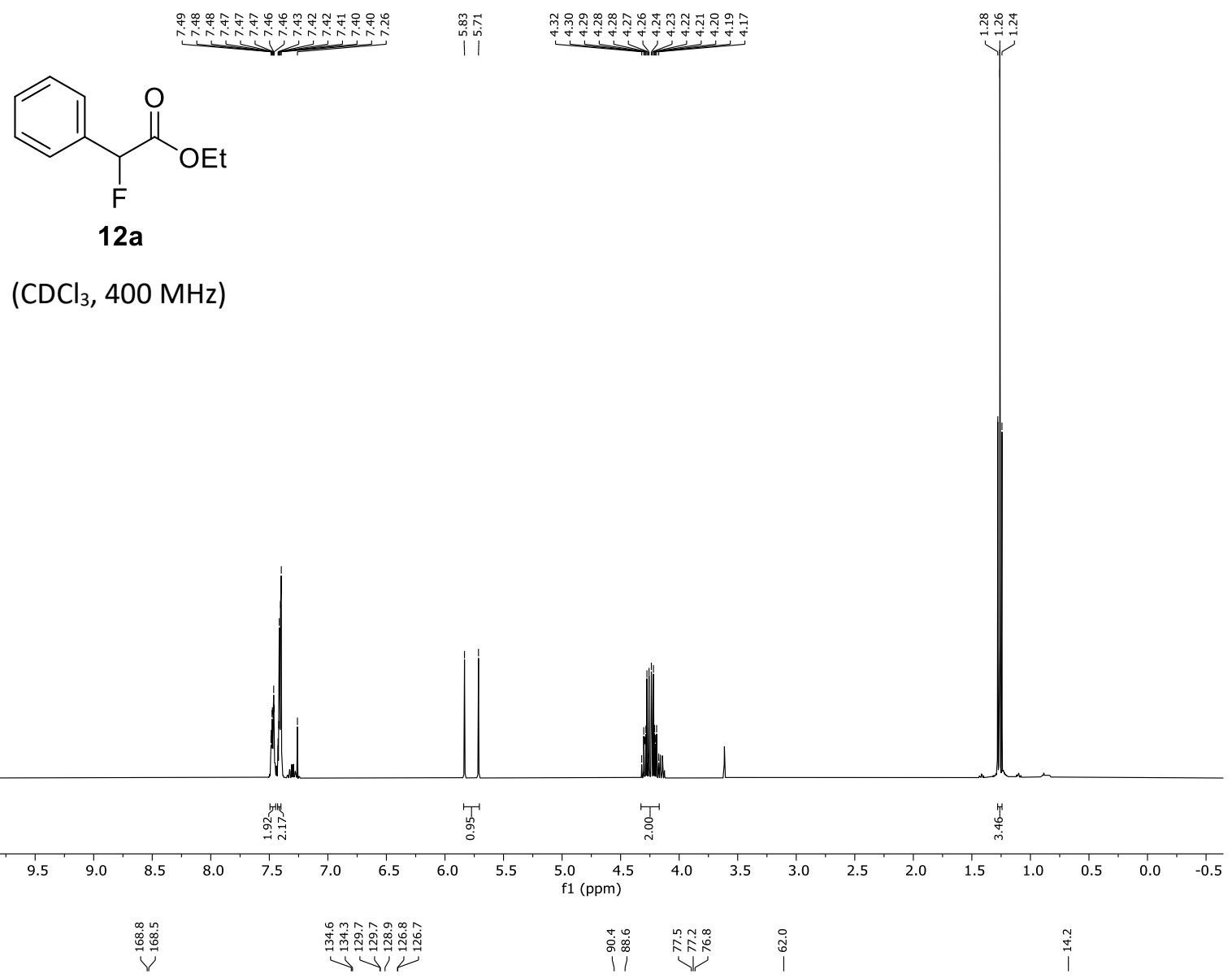

$\left(\mathrm{CDCl}_{3}, 100 \mathrm{MHz}\right)$

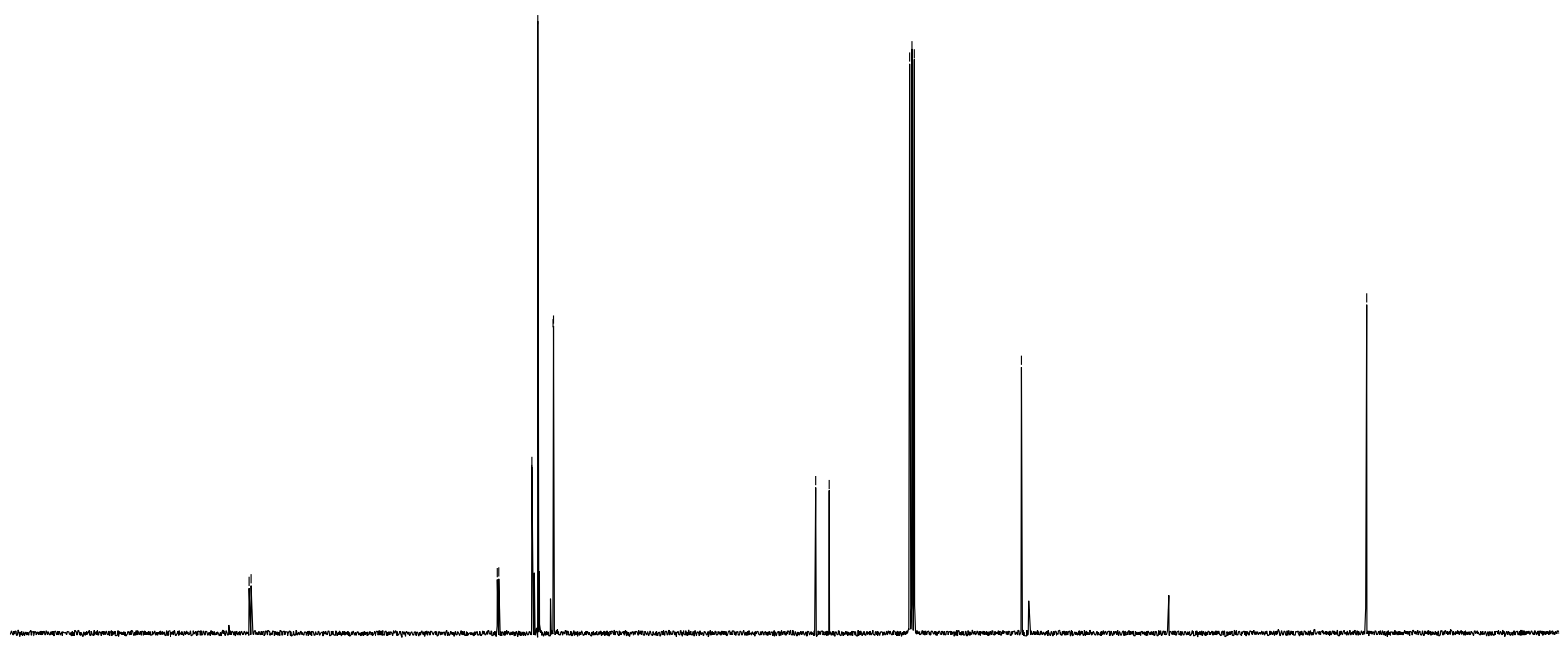

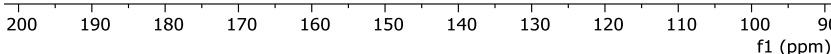


Divergent Synthesis of $\alpha$-Fluorinated Carbonyl and Carboxyl Derivatives by Double Electrophilic Activation of Amides

Internal

standard (fluorobenzene)

$\left(\mathrm{CDCl}_{3}, 376 \mathrm{MHz}\right)$

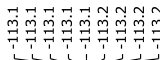

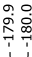

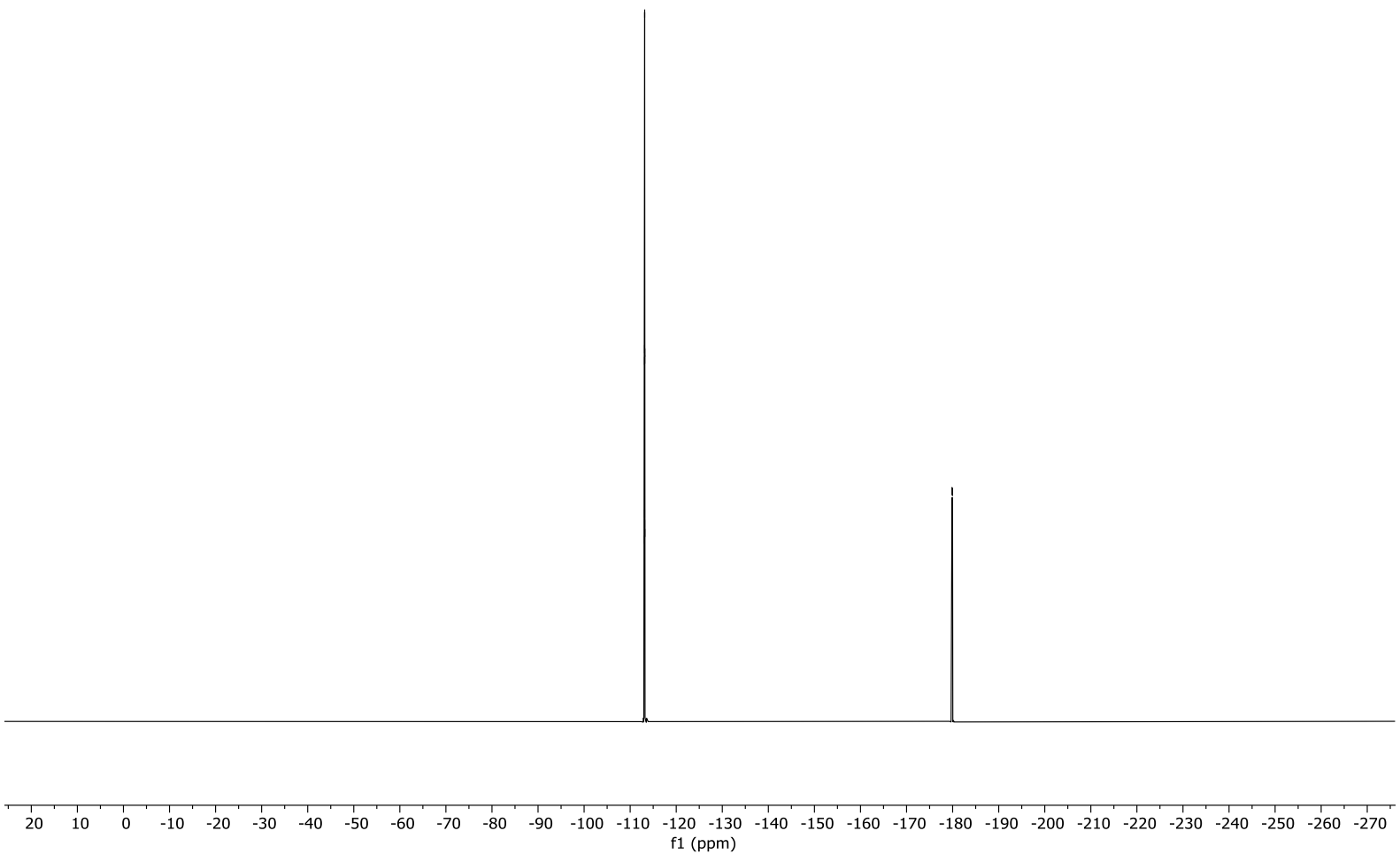




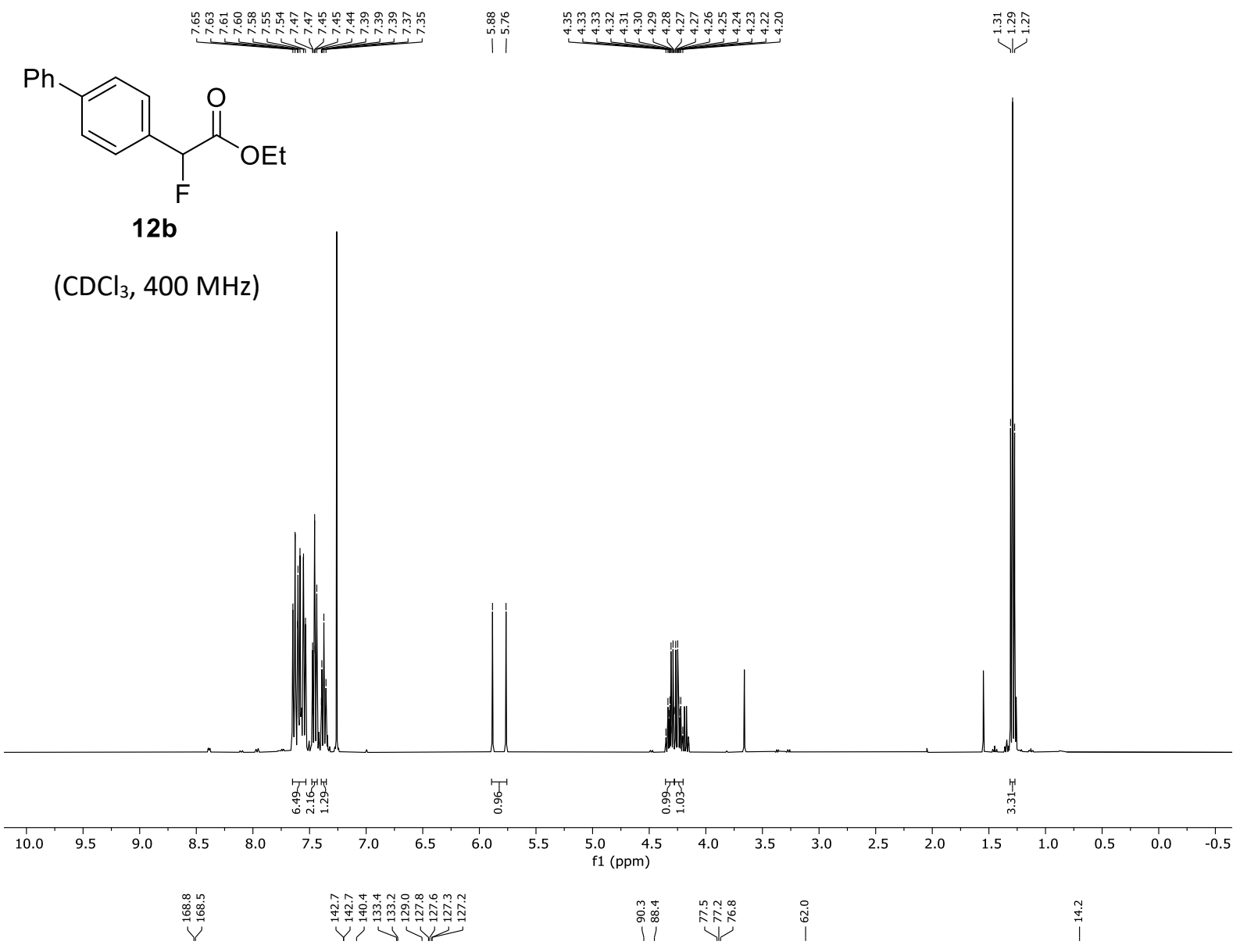

$\left(\mathrm{CDCl}_{3}, 100 \mathrm{MHz}\right)$

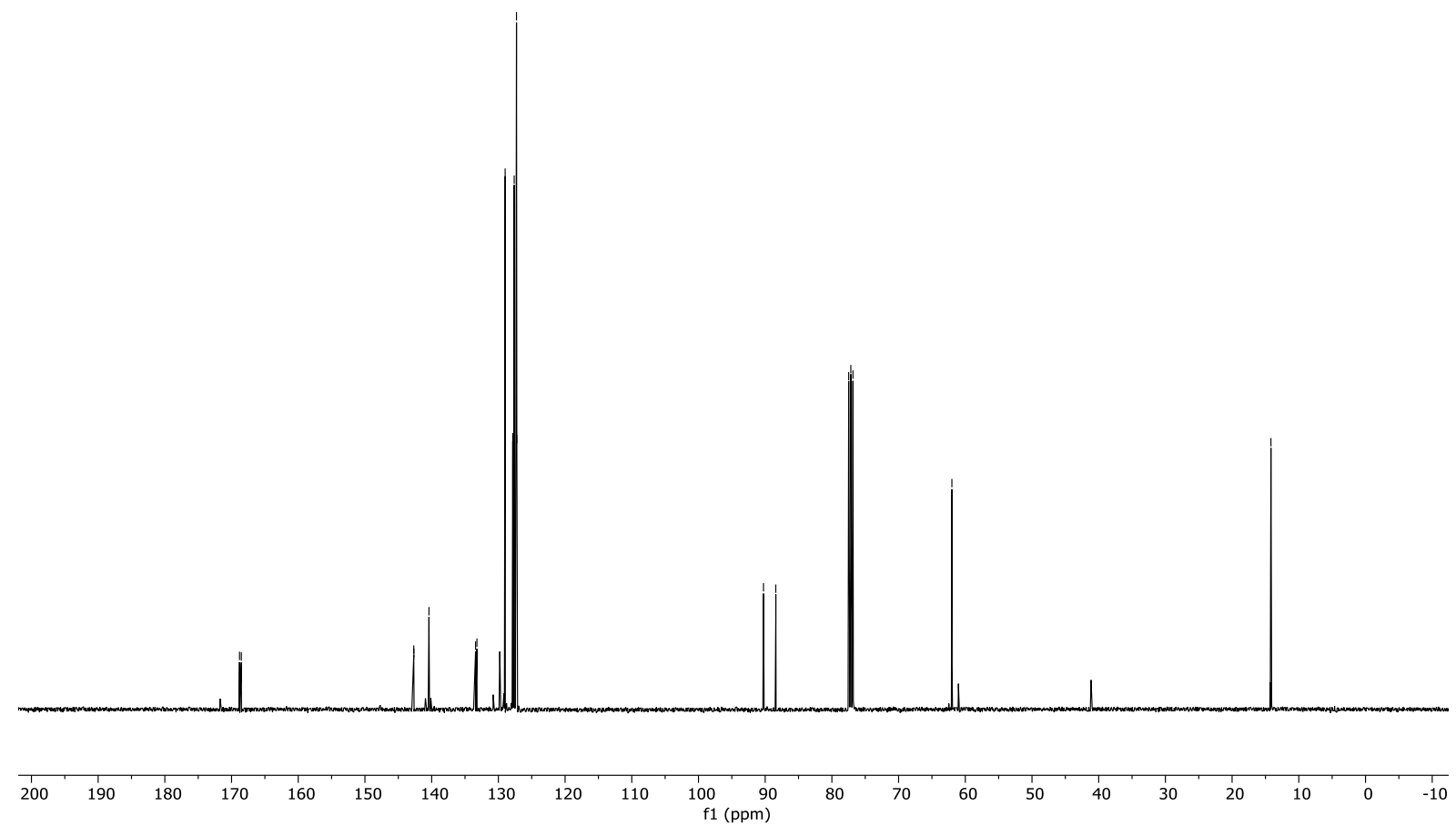


Divergent Synthesis of $\alpha$-Fluorinated Carbonyl and Carboxyl Derivatives by Double Electrophilic Activation of Amides

Internal

standard (fluorobenzene)

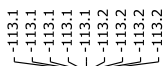

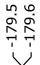

$\left(\mathrm{CDCl}_{3}, 376 \mathrm{MHz}\right)$

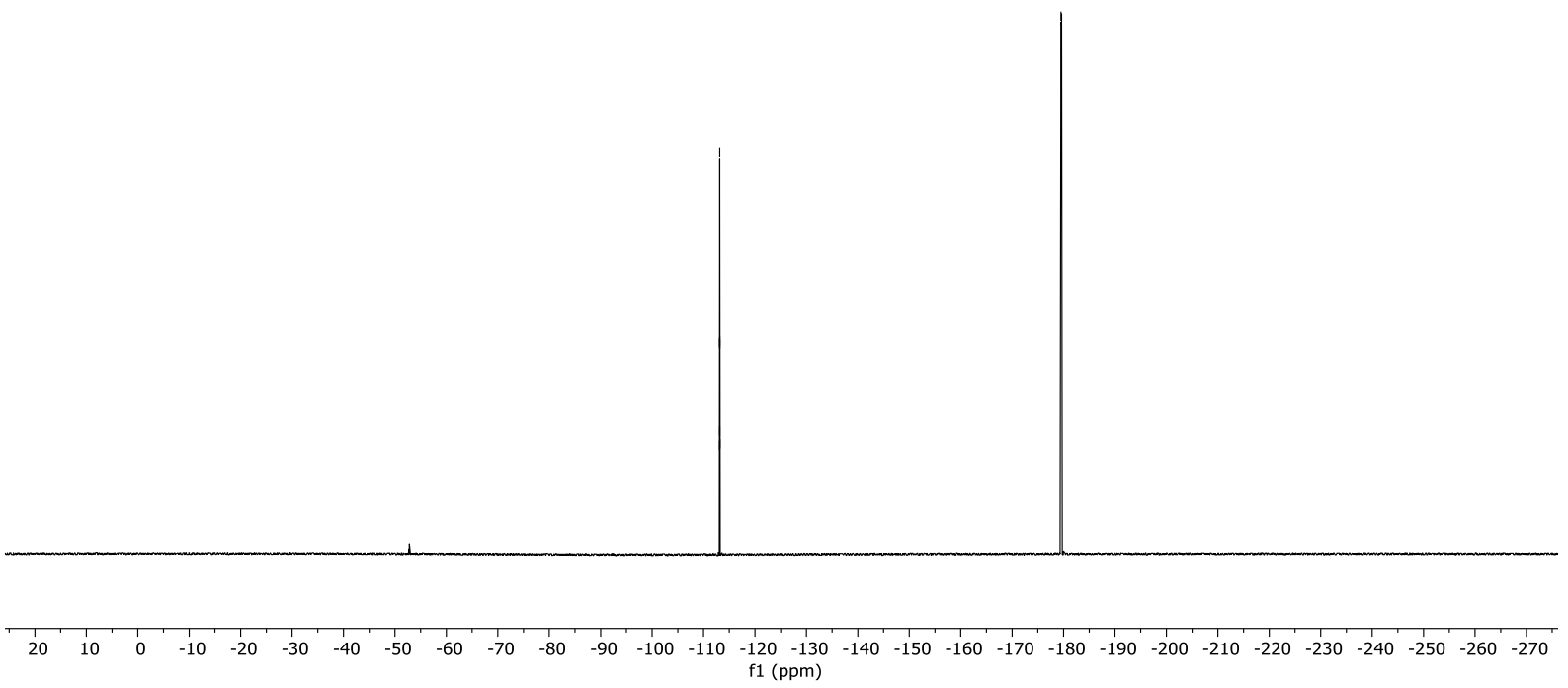




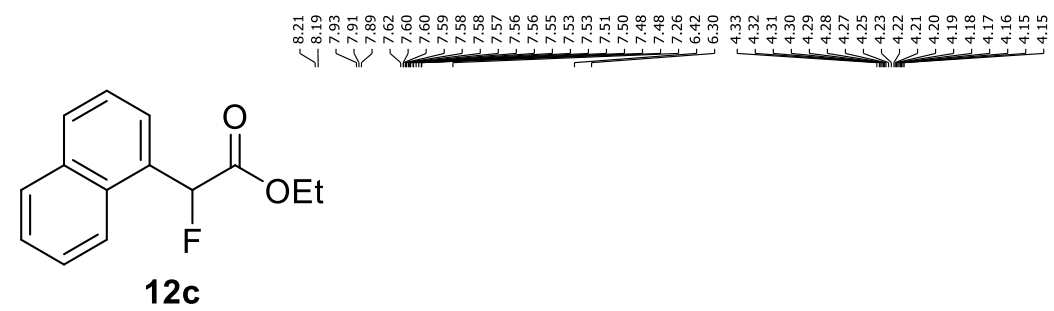

( $\left.\mathrm{CDCl}_{3}, 400 \mathrm{MHz}\right)$

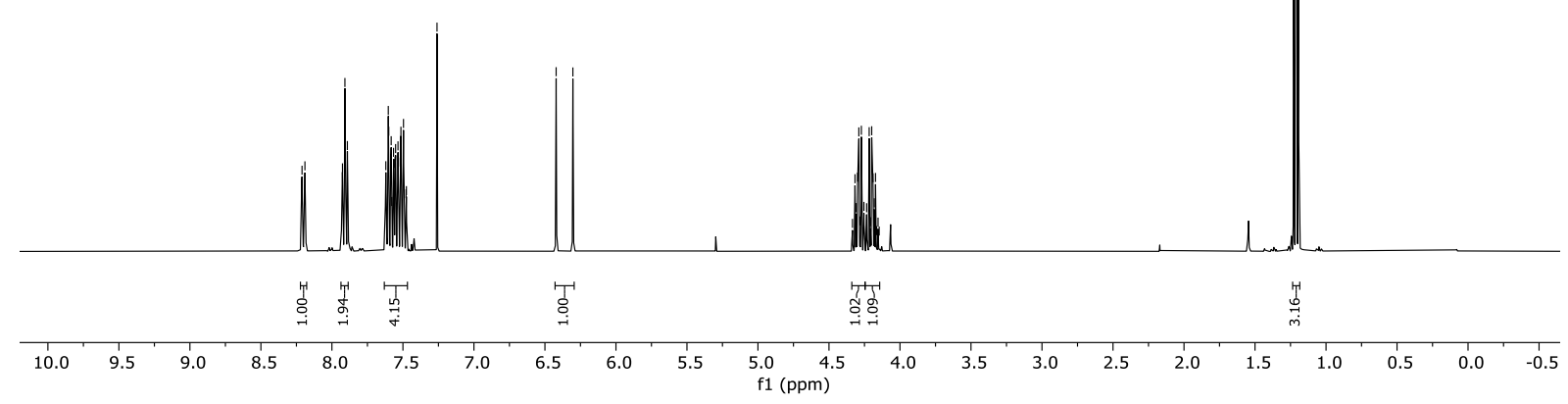

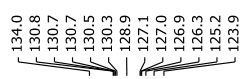

$\left(\mathrm{CDCl}_{3}, 100 \mathrm{MHz}\right)$

\section{}

$\stackrel{\sim}{\stackrel{\sim}{1}}$

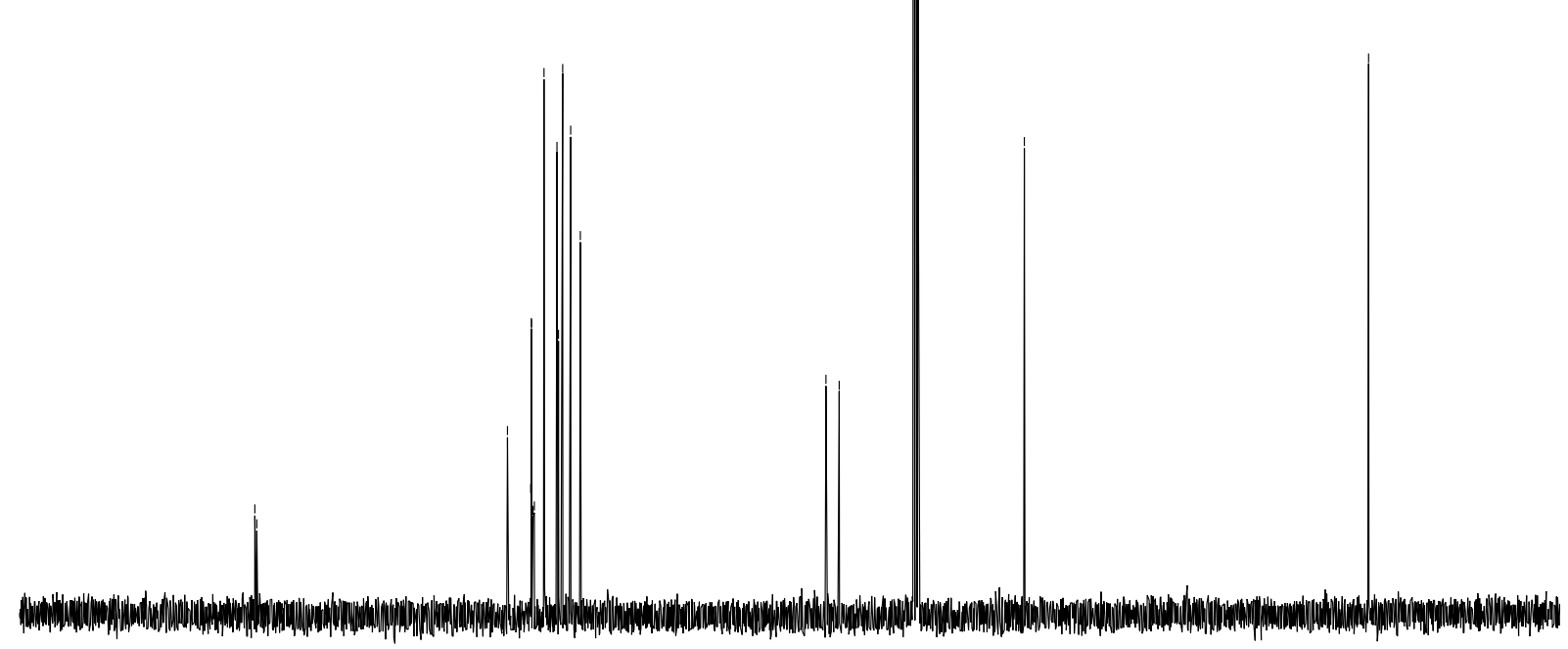

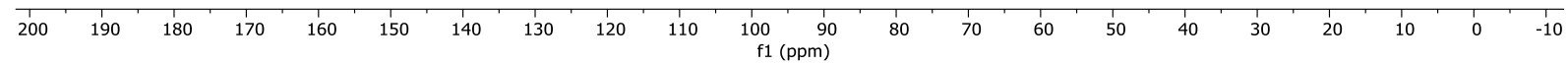


Divergent Synthesis of $\alpha$-Fluorinated Carbonyl and Carboxyl Derivatives by Double Electrophilic Activation of Amides

Internal

standard (fluorobenzene)

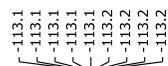

$\left(\mathrm{CDCl}_{3}, 376 \mathrm{MHz}\right)$

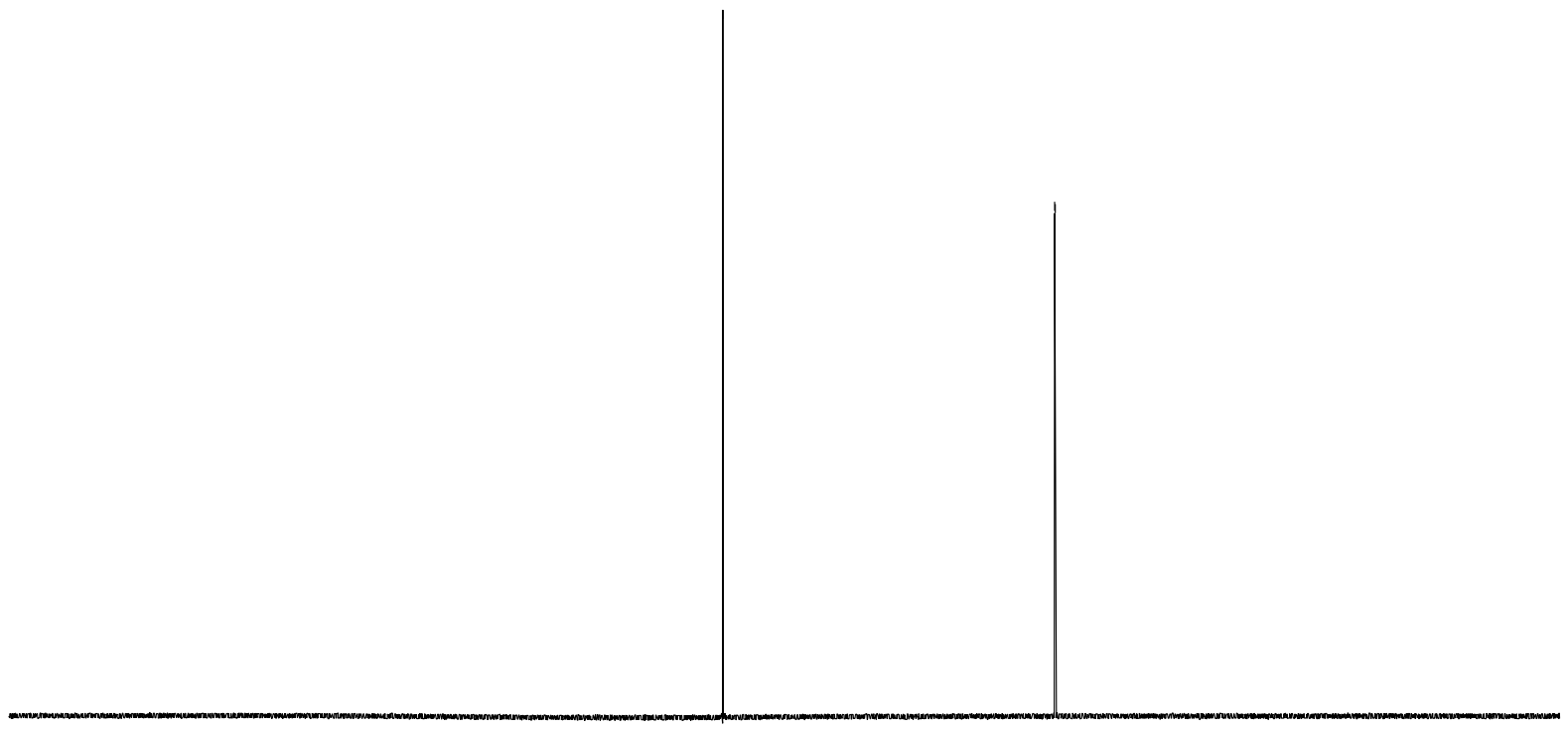

$\begin{array}{llllllllllllllllllllllllllllllllllllll}20 & 10 & 0 & -10 & -20 & -30 & -40 & -50 & -60 & -70 & -80 & -90 & -100 & -110 & -120 & -130 & -140 & -150 & -160 & -170 & -180 & -190 & -200 & -210 & -220 & -230 & -240 & -250 & -260 & -270\end{array}$ 


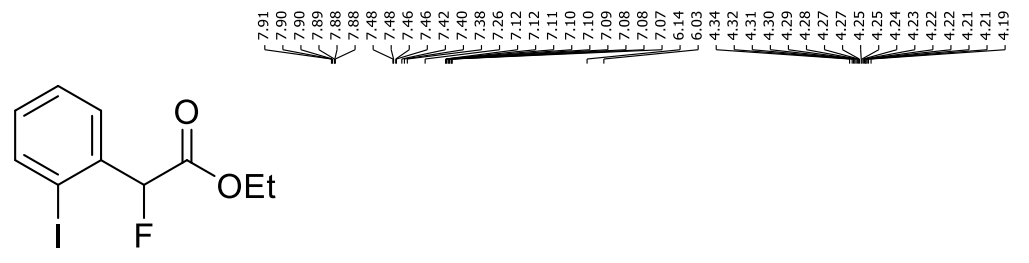

12d

$\left(\mathrm{CDCl}_{3}, 400 \mathrm{MHz}\right)$
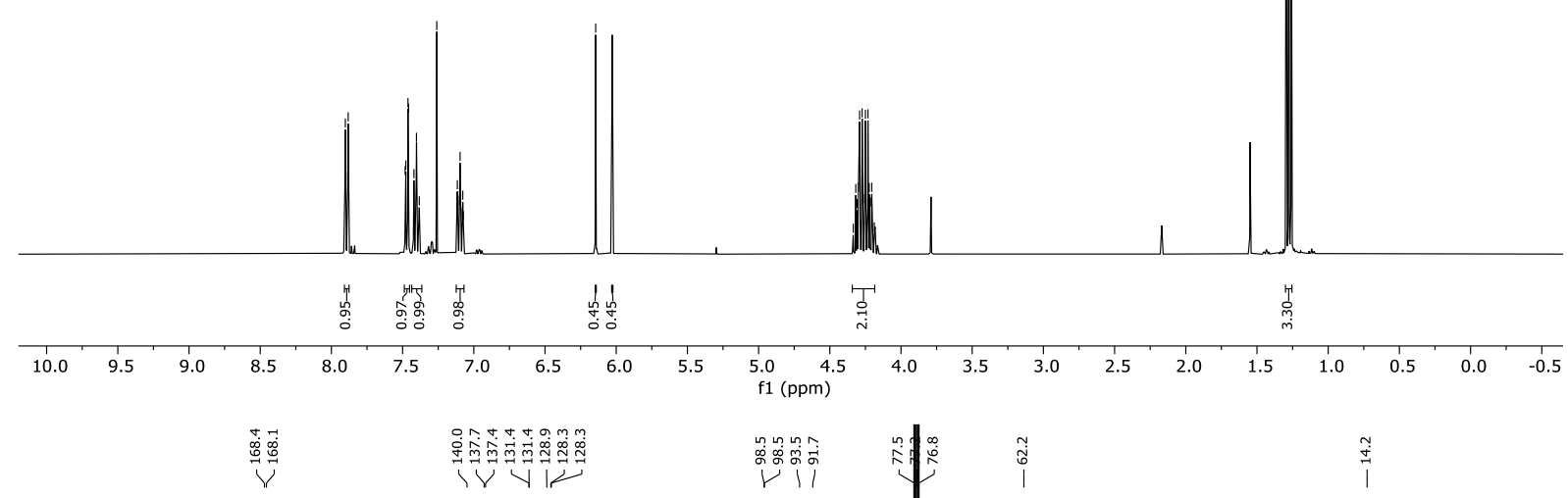

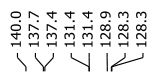

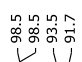

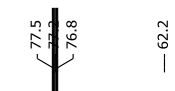

$\stackrel{1}{\stackrel{1}{*}}$

$\left(\mathrm{CDCl}_{3}, 100 \mathrm{MHz}\right)$

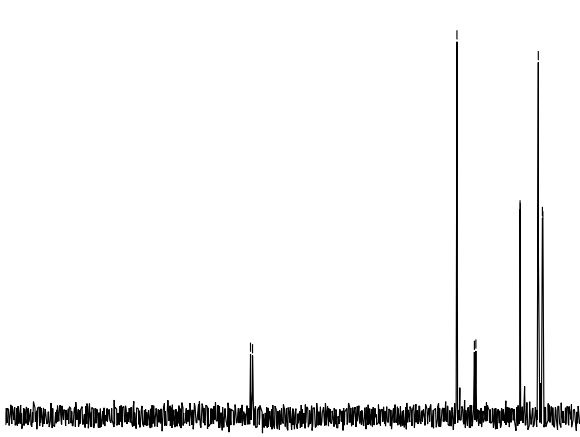

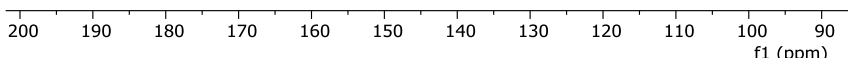


Divergent Synthesis of $\alpha$-Fluorinated Carbonyl and Carboxyl Derivatives by Double Electrophilic Activation of Amides

Internal

standard (fluorobenzene)

$\left(\mathrm{CDCl}_{3}, 376 \mathrm{MHz}\right)$

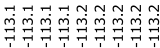

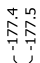

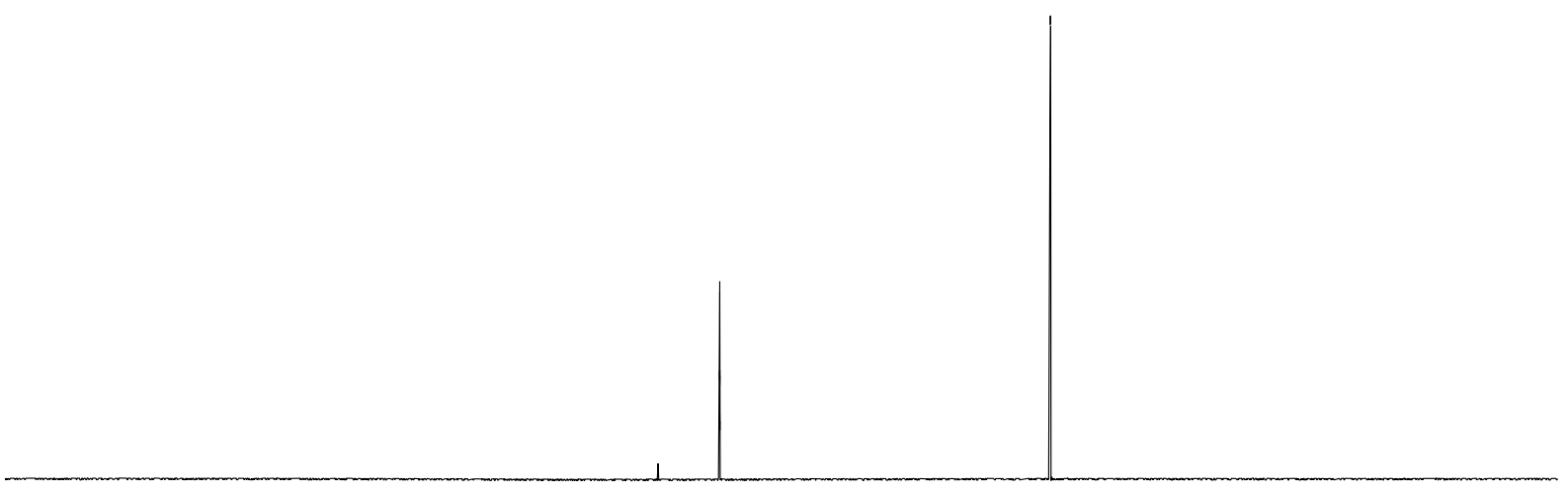

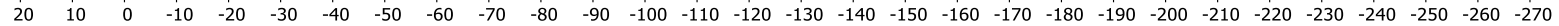
f1 (ppm) 


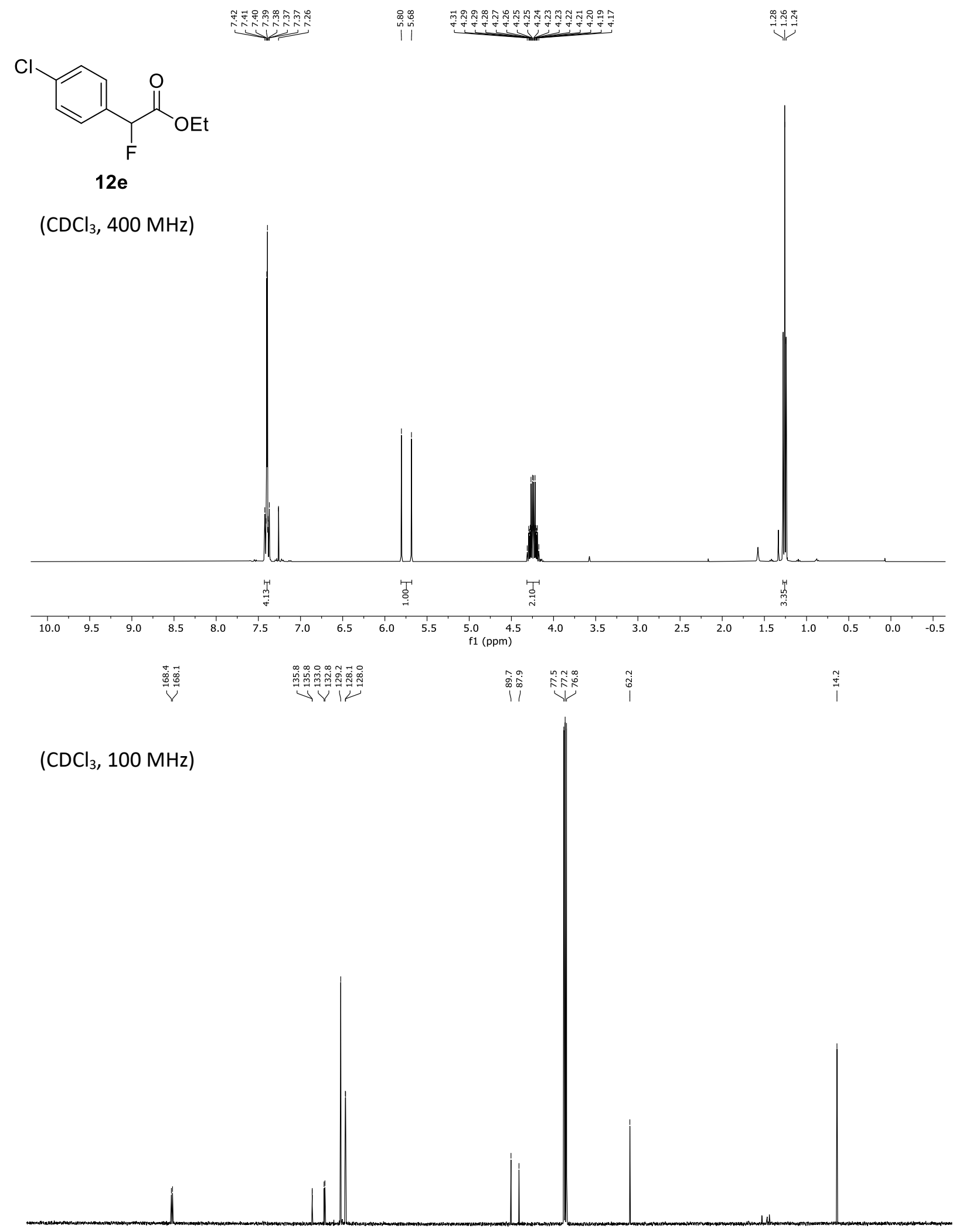

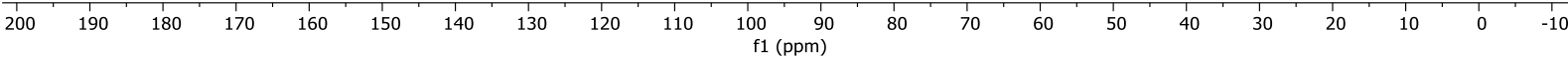


Divergent Synthesis of $\alpha$-Fluorinated Carbonyl and Carboxyl Derivatives by Double Electrophilic Activation of Amides

Internal

standard (fluorobenzene)

$\left(\mathrm{CDCl}_{3}, 376 \mathrm{MHz}\right)$

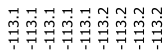

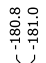

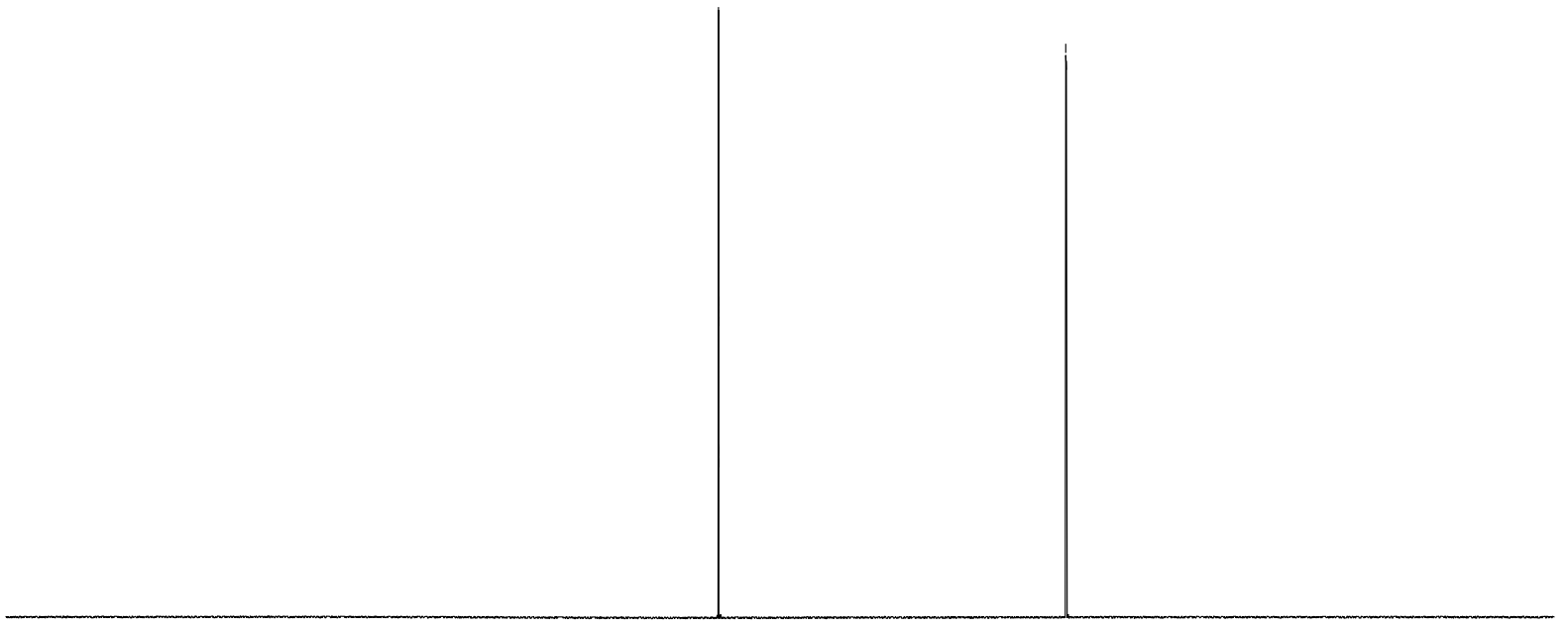

$\begin{array}{lllllllllllllllllllllllllllllllllllll}20 & 10 & 0 & -10 & -20 & -30 & -40 & -50 & -60 & -70 & -80 & -90 & -100 & -110 & -120 & -130 & -140 & -150 & -160 & -170 & -180 & -190 & -200 & -210 & -220 & -230 & -240 & -250 & -260 & -270\end{array}$ 


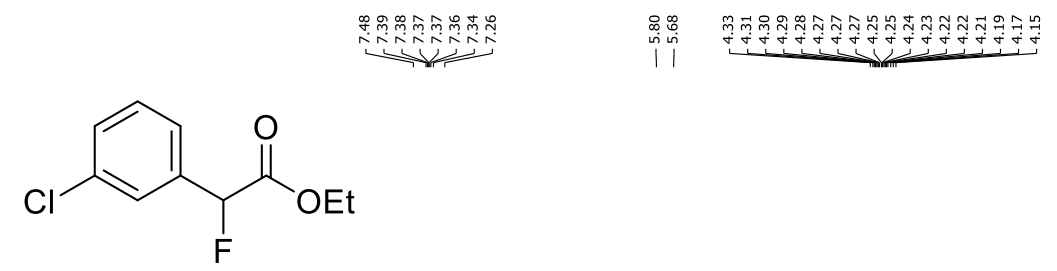

$12 f$

$\left(\mathrm{CDCl}_{3}, 400 \mathrm{MHz}\right.$ )

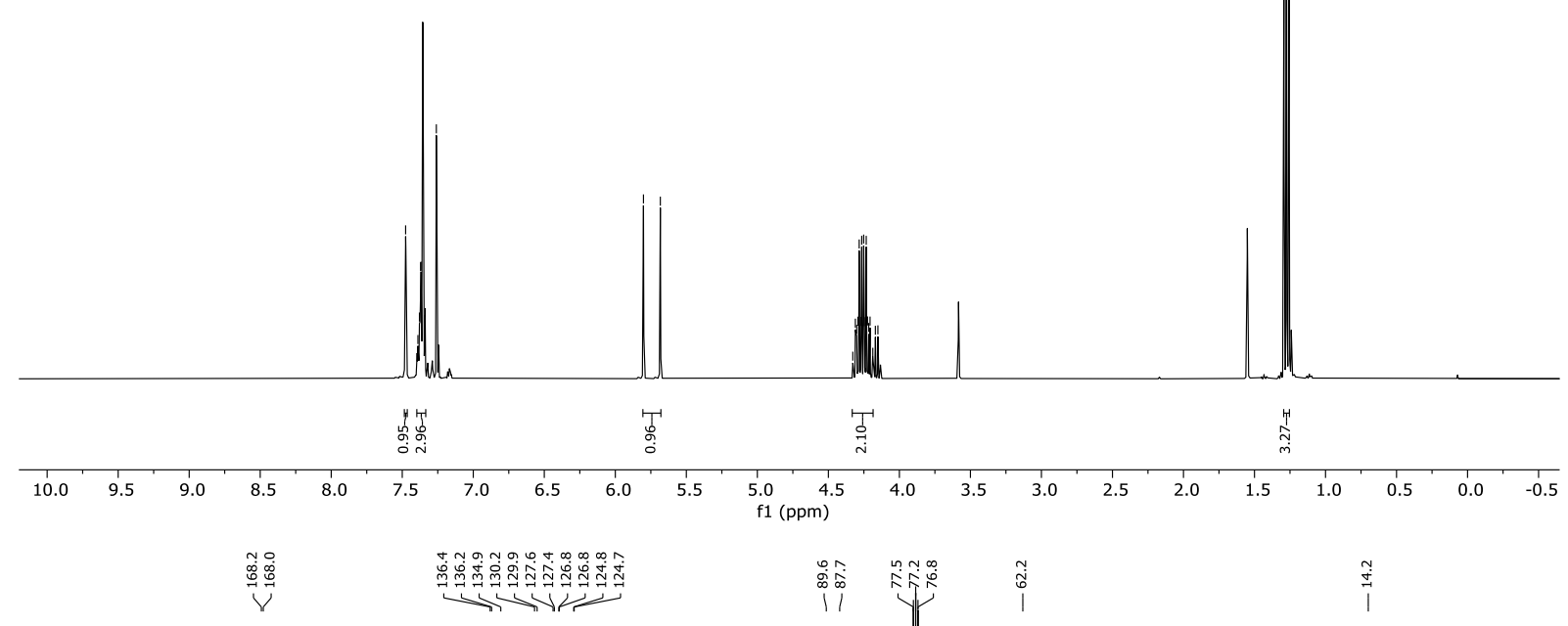

$\left(\mathrm{CDCl}_{3}, 100 \mathrm{MHz}\right.$ )

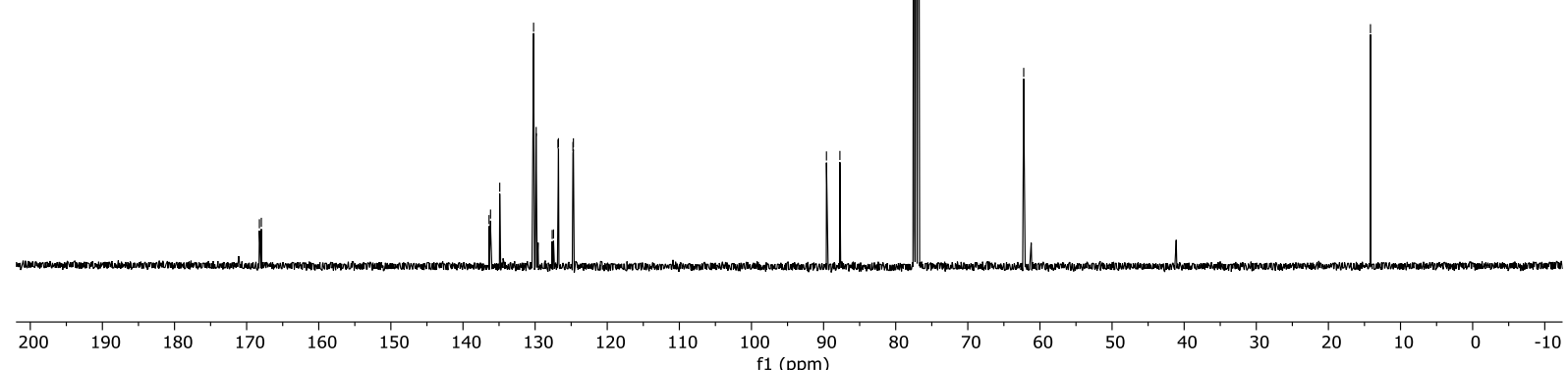


Divergent Synthesis of $\alpha$-Fluorinated Carbonyl and Carboxyl Derivatives by Double Electrophilic Activation of Amides

Internal

standard (fluorobenzene)

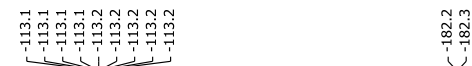

$\left(\mathrm{CDCl}_{3}, 376 \mathrm{MHz}\right)$

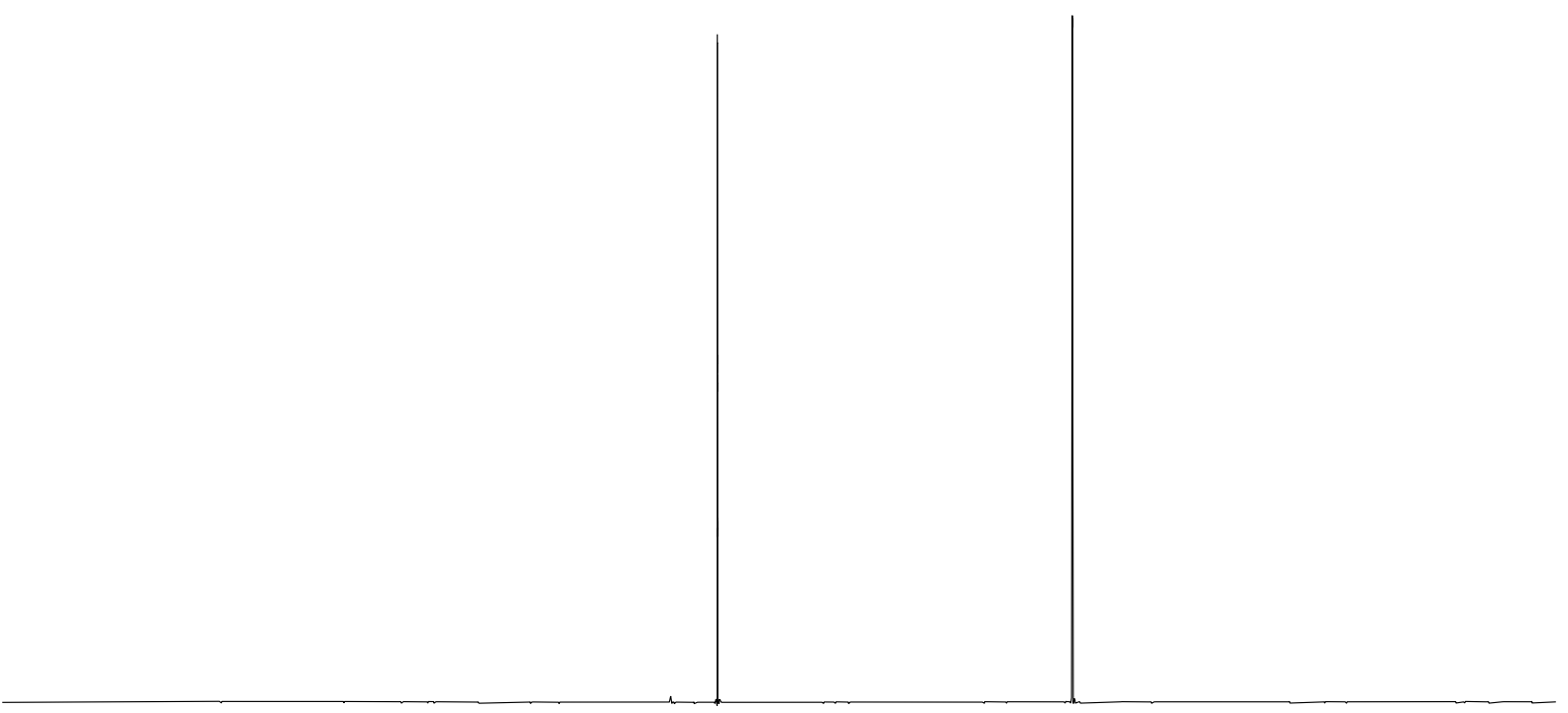

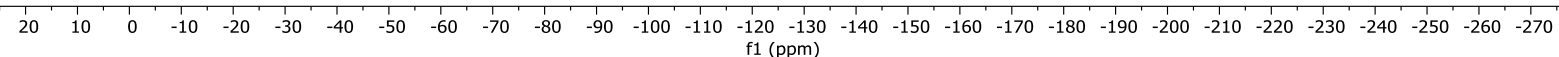




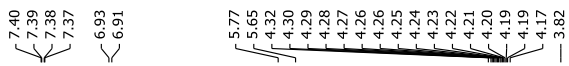<smiles>CCOC(=O)C(F)c1ccc(OC)cc1</smiles>

$12 \mathrm{~g}$

$\left(\mathrm{CDCl}_{3}, 400 \mathrm{MHz}\right)$

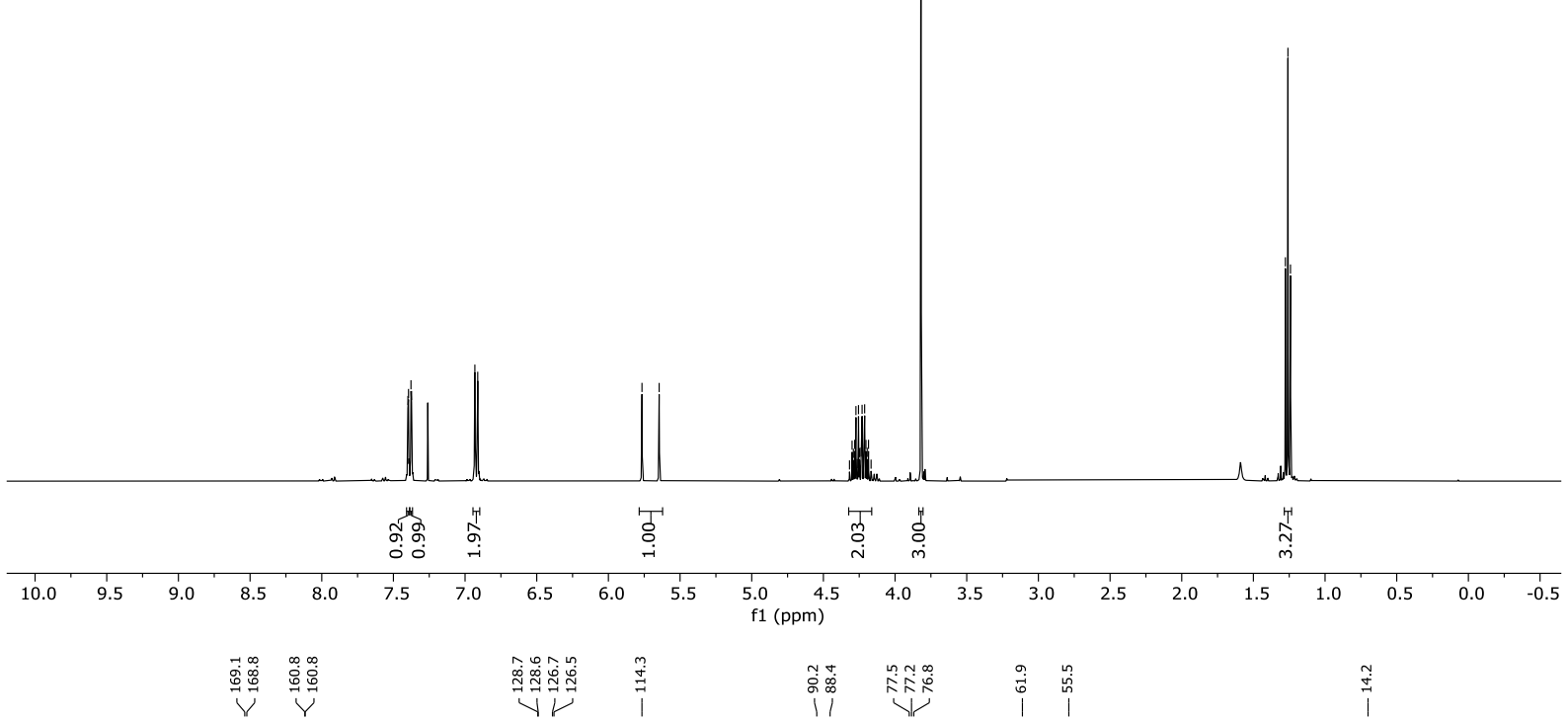

$\left(\mathrm{CDCl}_{3}, 100 \mathrm{MHz}\right)$

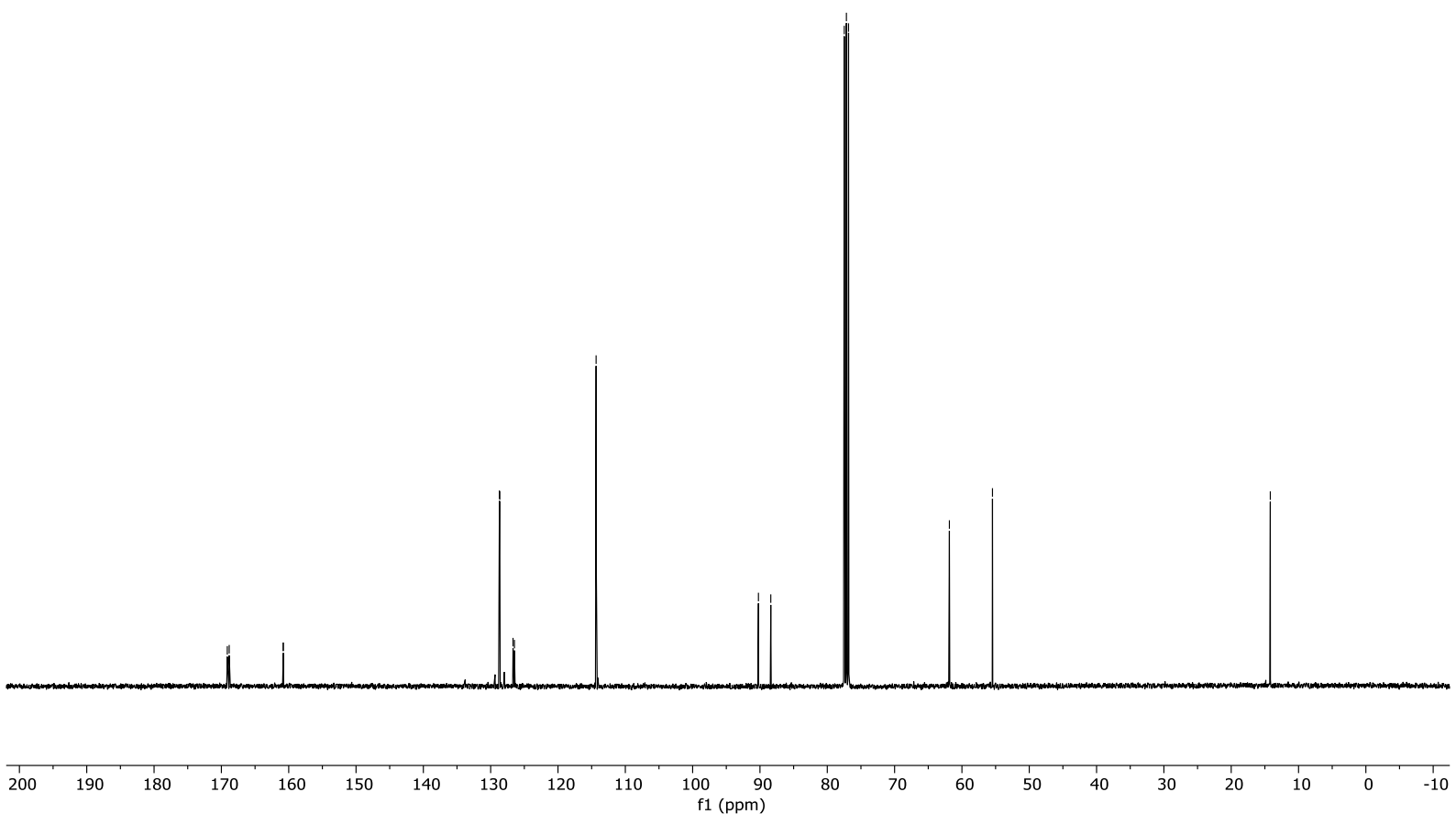


Divergent Synthesis of $\alpha$-Fluorinated Carbonyl and Carboxyl Derivatives by Double Electrophilic Activation of Amides

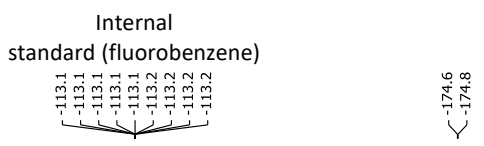

$\left(\mathrm{CDCl}_{3}, 376 \mathrm{MHz}\right)$

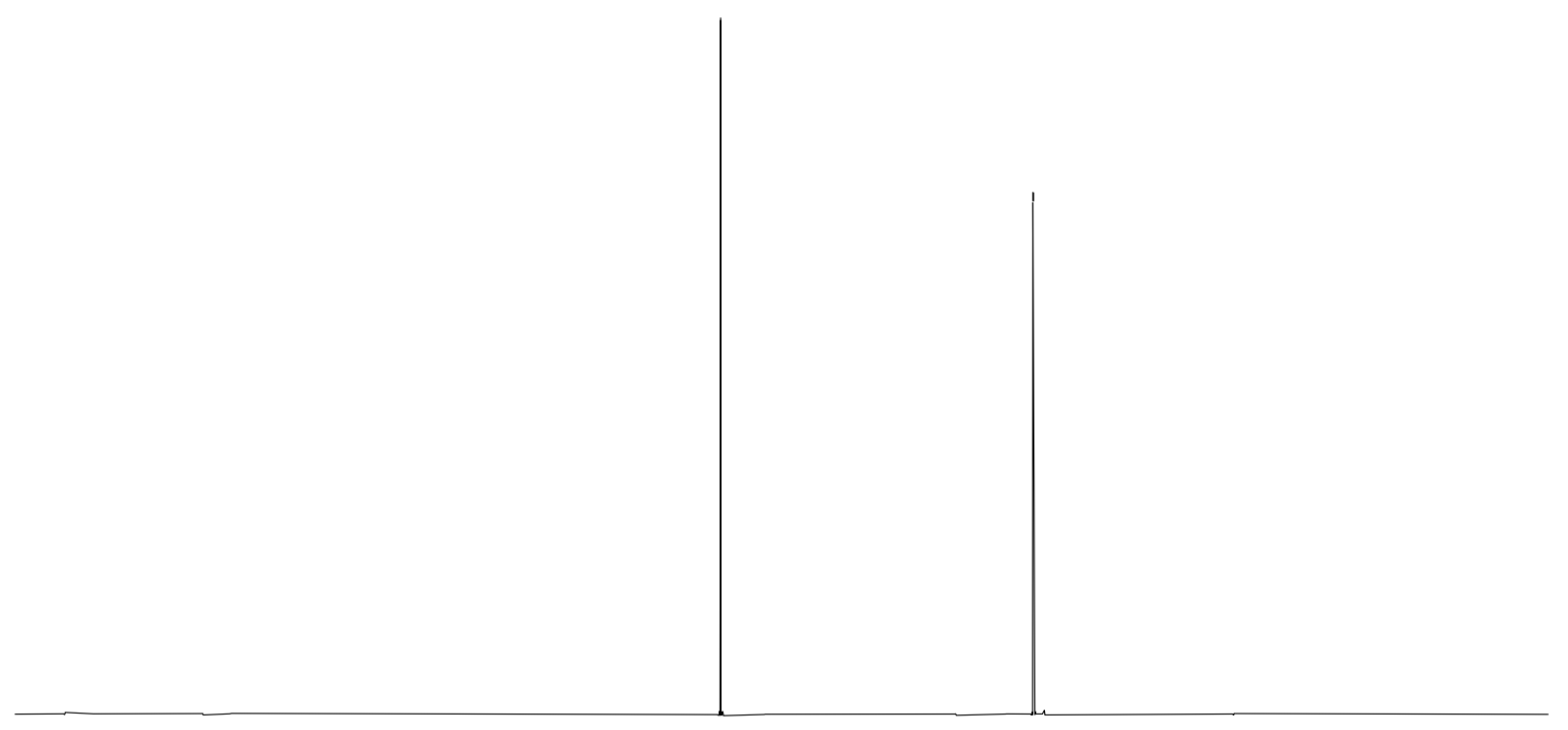

$\begin{array}{lllllllllllllllllllllllllllllllll}20 & 10 & 0 & -10 & -20 & -30 & -40 & -50 & -60 & -70 & -80 & -90 & -100 & -110 & -120 & -130 & -140 & -150 & -160 & -170 & -180 & -190 & -200 & -210 & -220 & -230 & -240 & -250 & -260 & -270\end{array}$ f1 (ppm) 
<smiles>C=C(OCC)C(=O)OCC</smiles>

$12 \mathrm{~h}$

$\left(\mathrm{CDCl}_{3}, 400 \mathrm{MHz}\right)$

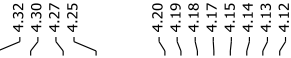

$-\mathrm{CH}_{2} \mathrm{CO}_{2} \mathrm{CH}_{2} \mathrm{CH}_{3}$

$-\mathrm{CHF}-\mathrm{CO}_{2} \mathrm{CH}_{2} \mathrm{CH}_{3}$ M

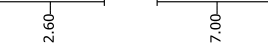

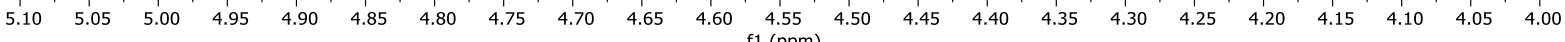

Zoom on the ${ }^{1} \mathrm{H}$ NMR spectra of crude reaction mixture containing $12 \%$ of fluorinated ester $12 \mathrm{~h}$ and $42 \%$ of the corresponding non-fluorinated ester. Only characteristics and well separated signals were integrated in the NMR spectra. ${ }^{1} \mathrm{H}$ NMR yield determined using 1,1,2,2-tetrachloroethane as an internal standard. 


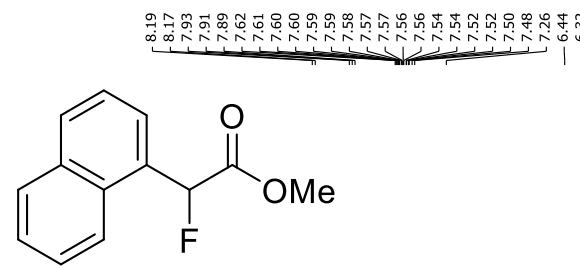

$12 \mathbf{i}$

$\left(\mathrm{CDCl}_{3}, 400 \mathrm{MHz}\right)$
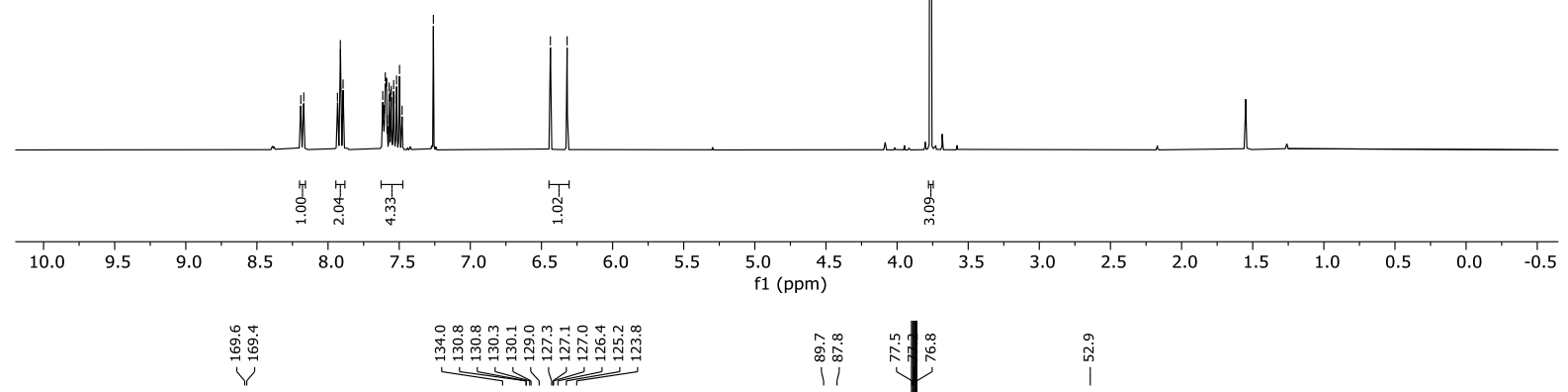

$\left(\mathrm{CDCl}_{3}, 100 \mathrm{MHz}\right)$

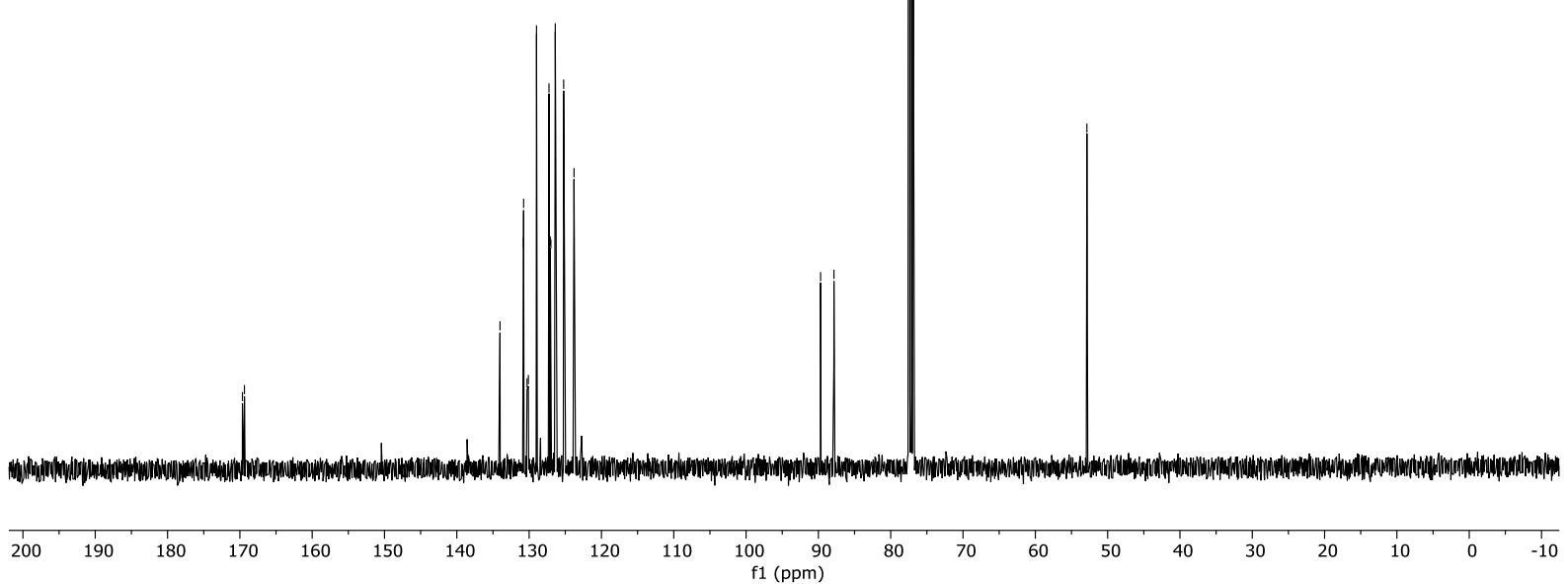


Divergent Synthesis of $\alpha$-Fluorinated Carbonyl and Carboxyl Derivatives by Double Electrophilic Activation of Amides

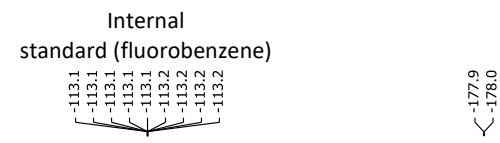

$\left(\mathrm{CDCl}_{3}, 376 \mathrm{MHz}\right)$

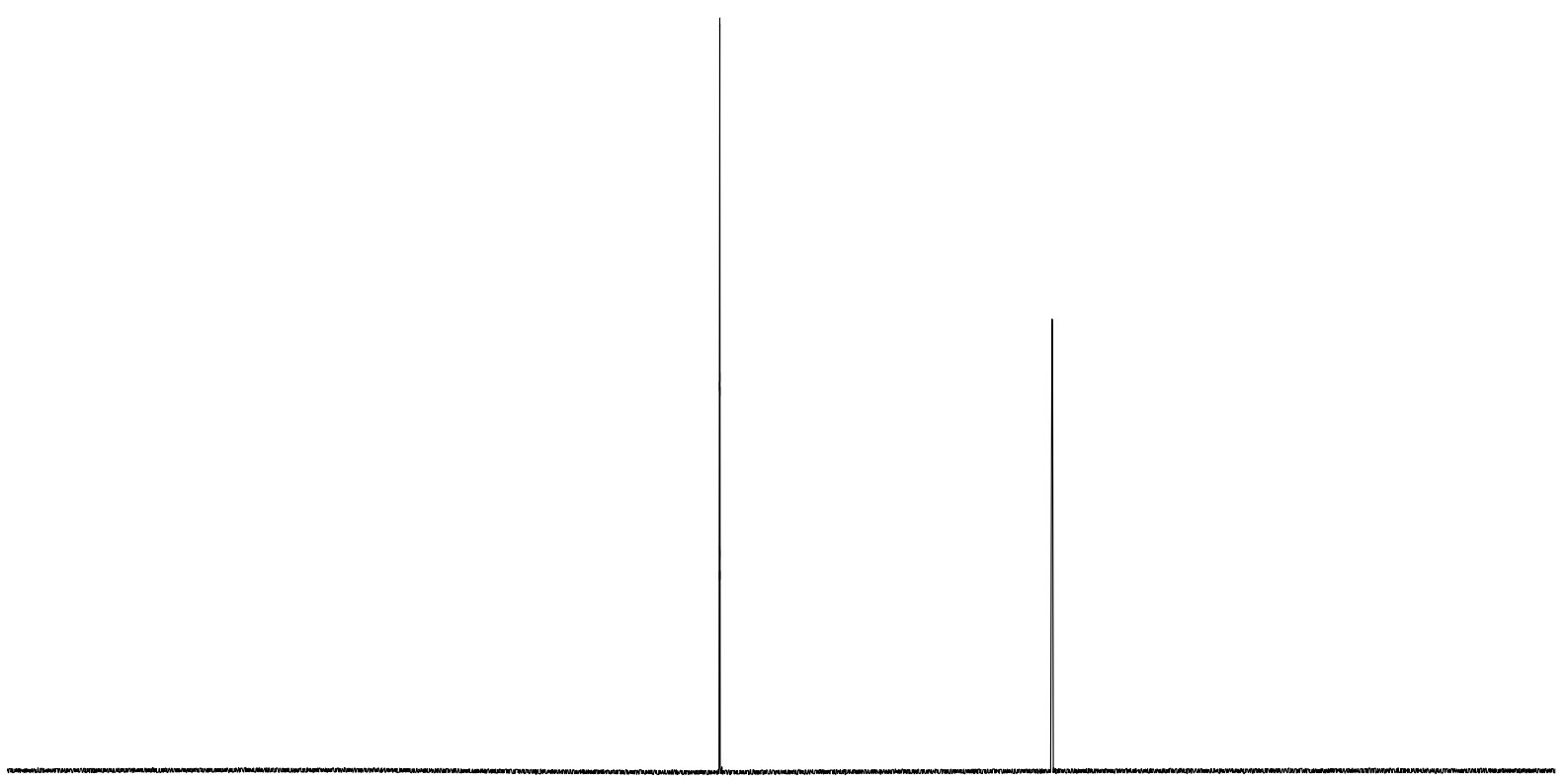

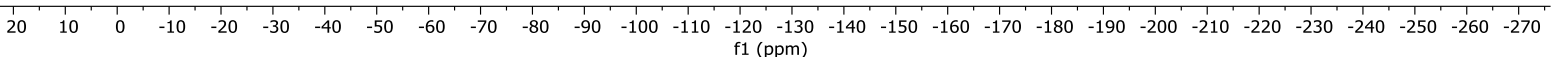



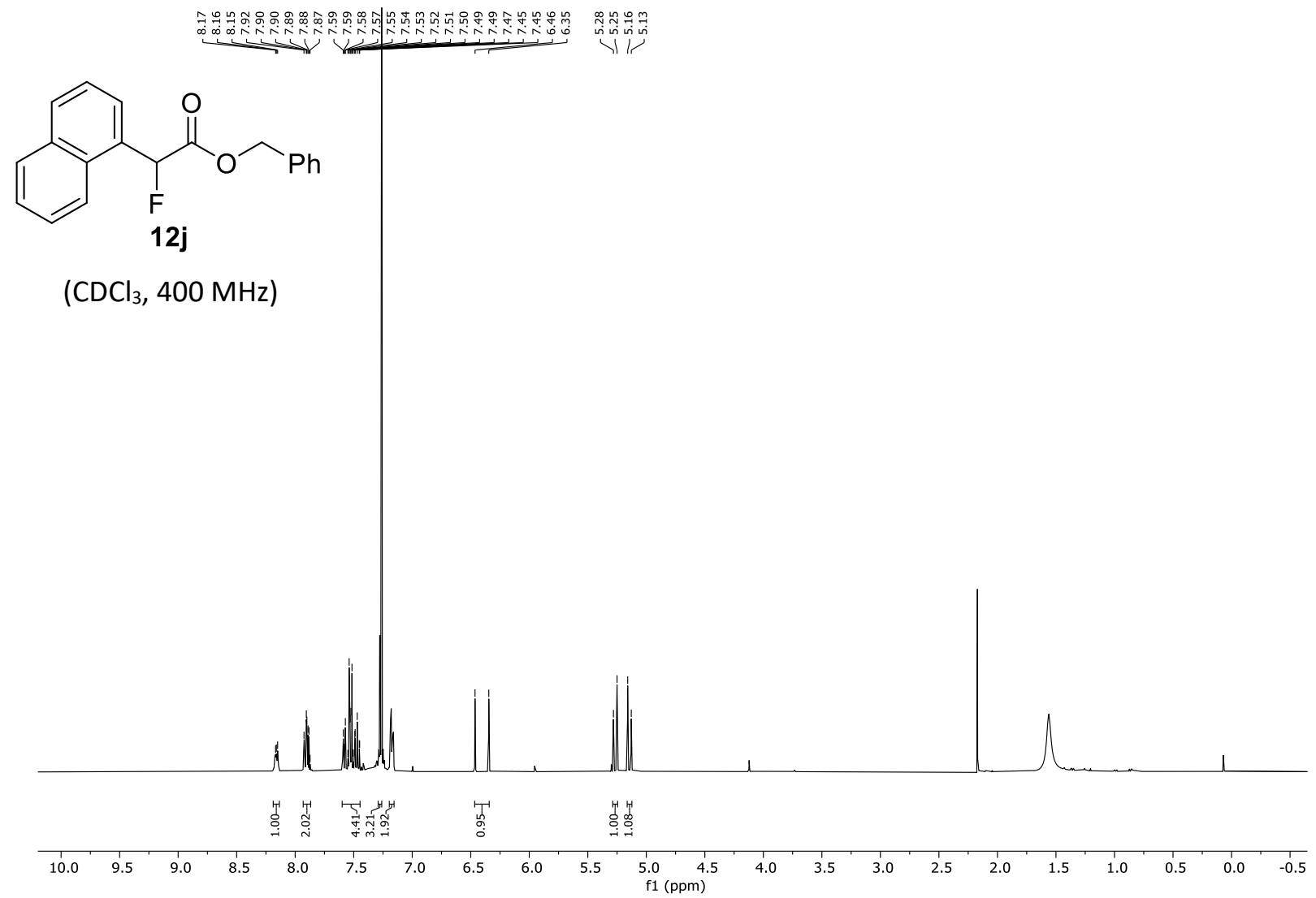

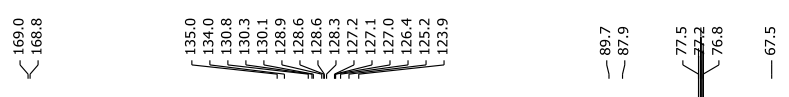

$\left(\mathrm{CDCl}_{3}, 100 \mathrm{MHz}\right)$

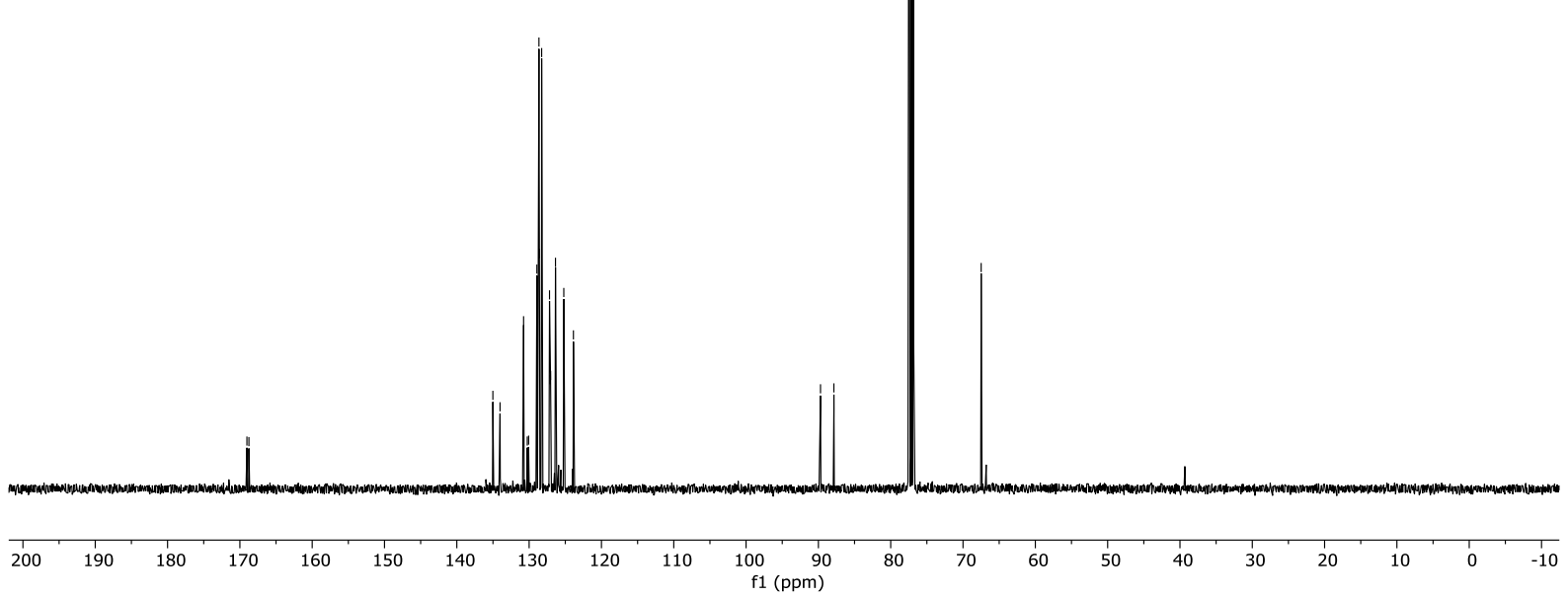


Divergent Synthesis of $\alpha$-Fluorinated Carbonyl and Carboxyl Derivatives by Double Electrophilic Activation of Amides

\section{standard (fluorobenzene)}

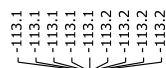

$\left(\mathrm{CDCl}_{3}, 376 \mathrm{MHz}\right)$

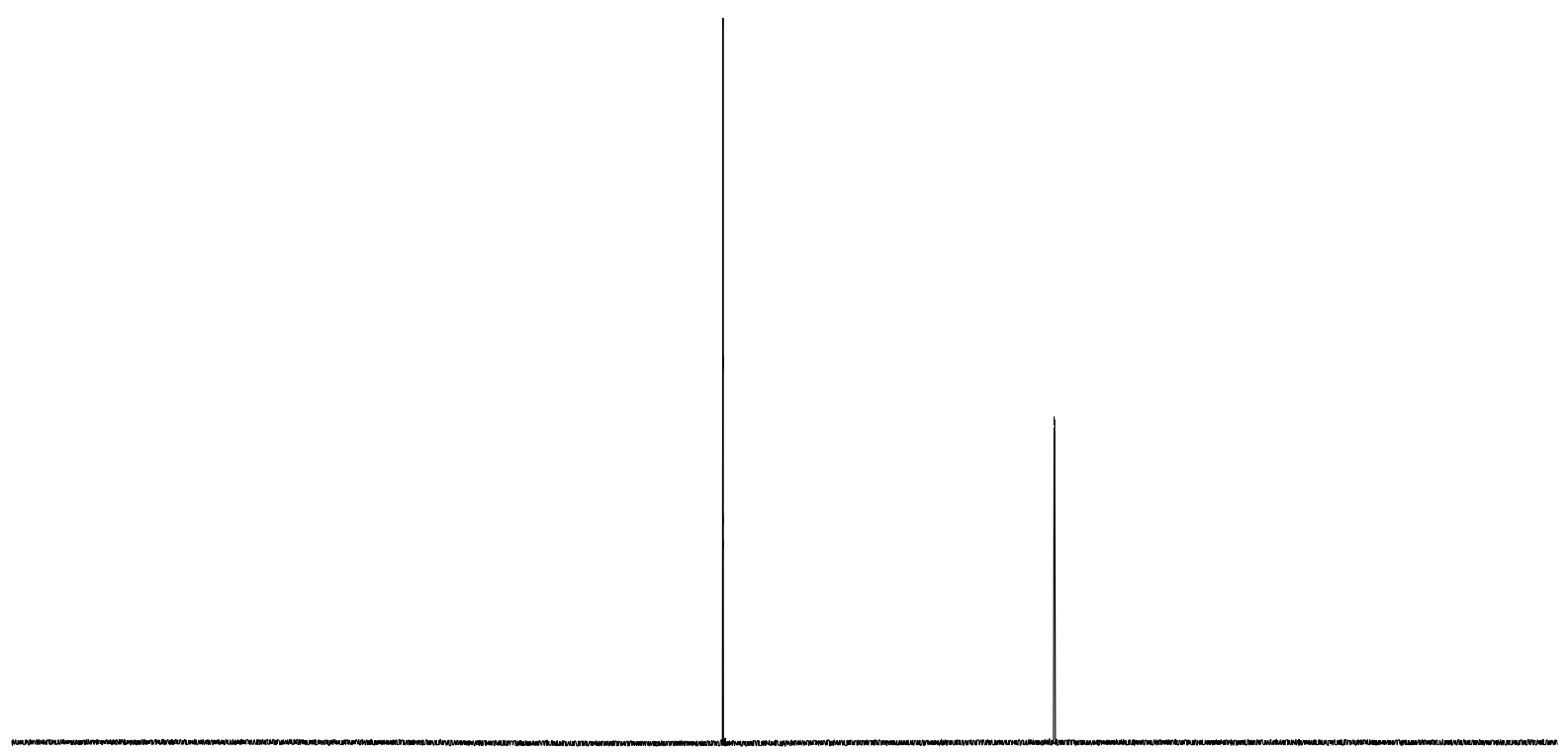

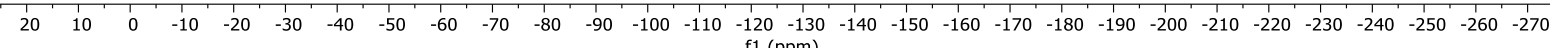
f1 (ppm) 


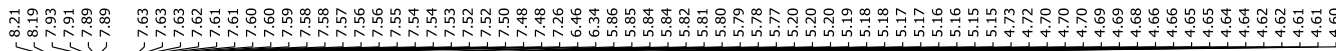<smiles>C=CCOC(=O)C(F)c1cccc2ccccc12</smiles>

$12 k$

$\left(\mathrm{CDCl}_{3}, 400 \mathrm{MHz}\right)$

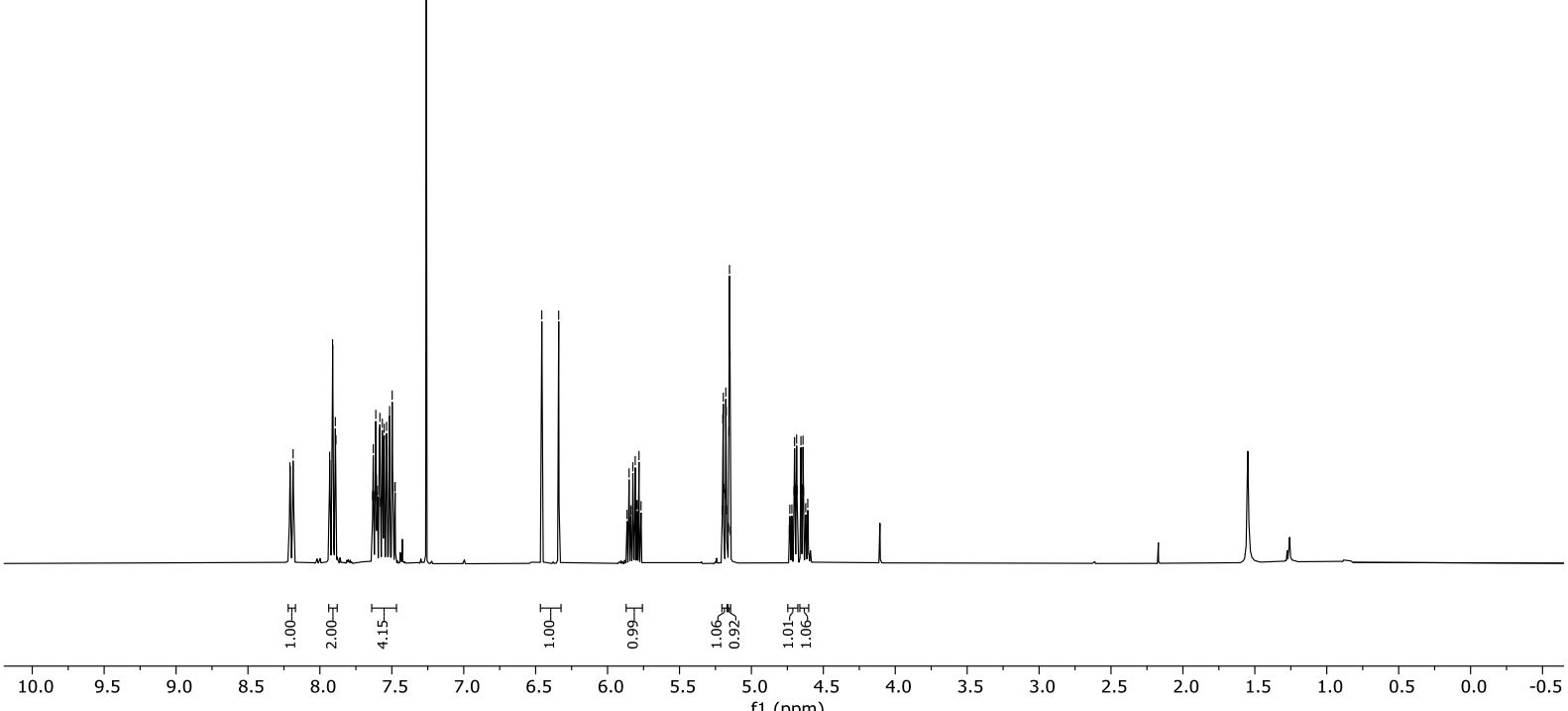

V

$\left(\mathrm{CDCl}_{3}, 100 \mathrm{MHz}\right)$

\begin{tabular}{lllllllllllllllllllllllllll}
\hline & 190 & 180 & 170 & 160 & 150 & 140 & 130 & 120 & 110 & 100 & 90 & 80 & 70 & 60 & 50 & 40 & 30 & 20 & 10 & 0 & -10
\end{tabular} 
Divergent Synthesis of $\alpha$-Fluorinated Carbonyl and Carboxyl Derivatives by Double Electrophilic Activation of Amides

\section{$\begin{aligned} \text { Internal } & \text { standard (fluorobenzene) }\end{aligned}$

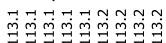

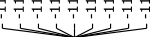

$\left(\mathrm{CDCl}_{3}, 376 \mathrm{MHz}\right)$

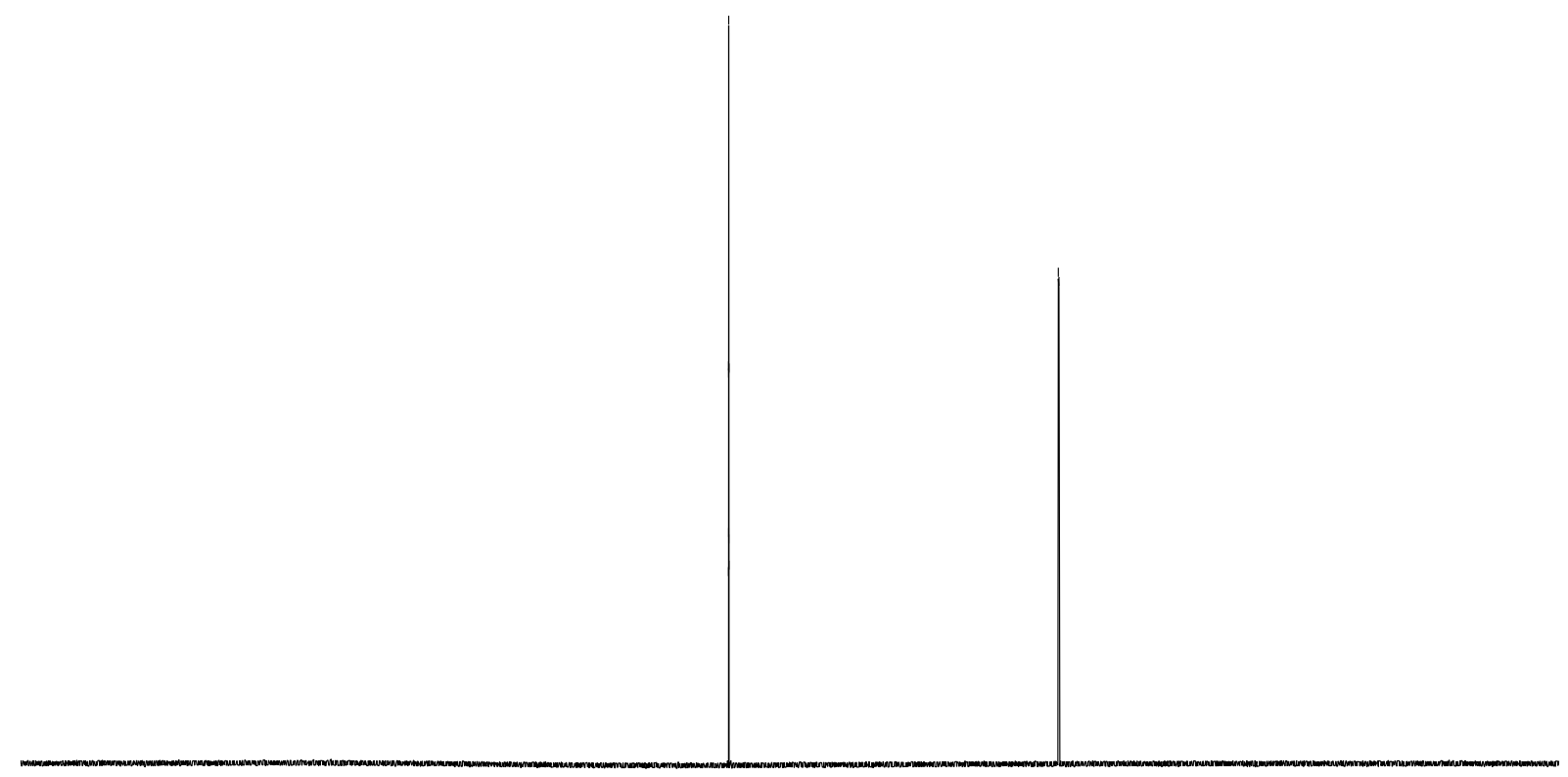

$\begin{array}{lllllllllllllllllllllllllllllllllllll}20 & 10 & 0 & -10 & -20 & -30 & -40 & -50 & -60 & -70 & -80 & -90 & -100 & -110 & -120 & -130 & -140 & -150 & -160 & -170 & -180 & -190 & -200 & -210 & -220 & -230 & -240 & -250 & -260 & -270\end{array}$ 

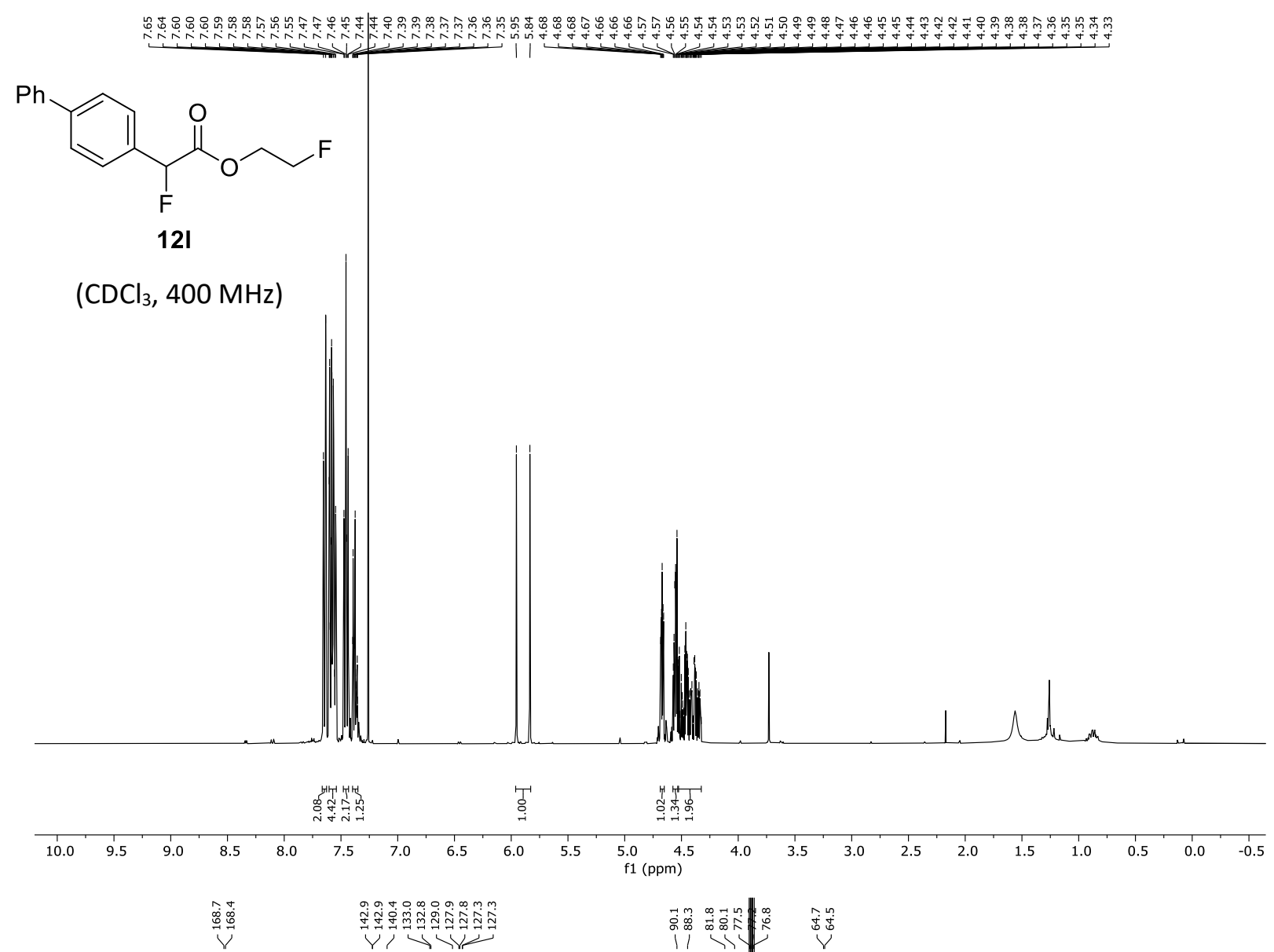

$\left(\mathrm{CDCl}_{3}, 100 \mathrm{MHz}\right)$

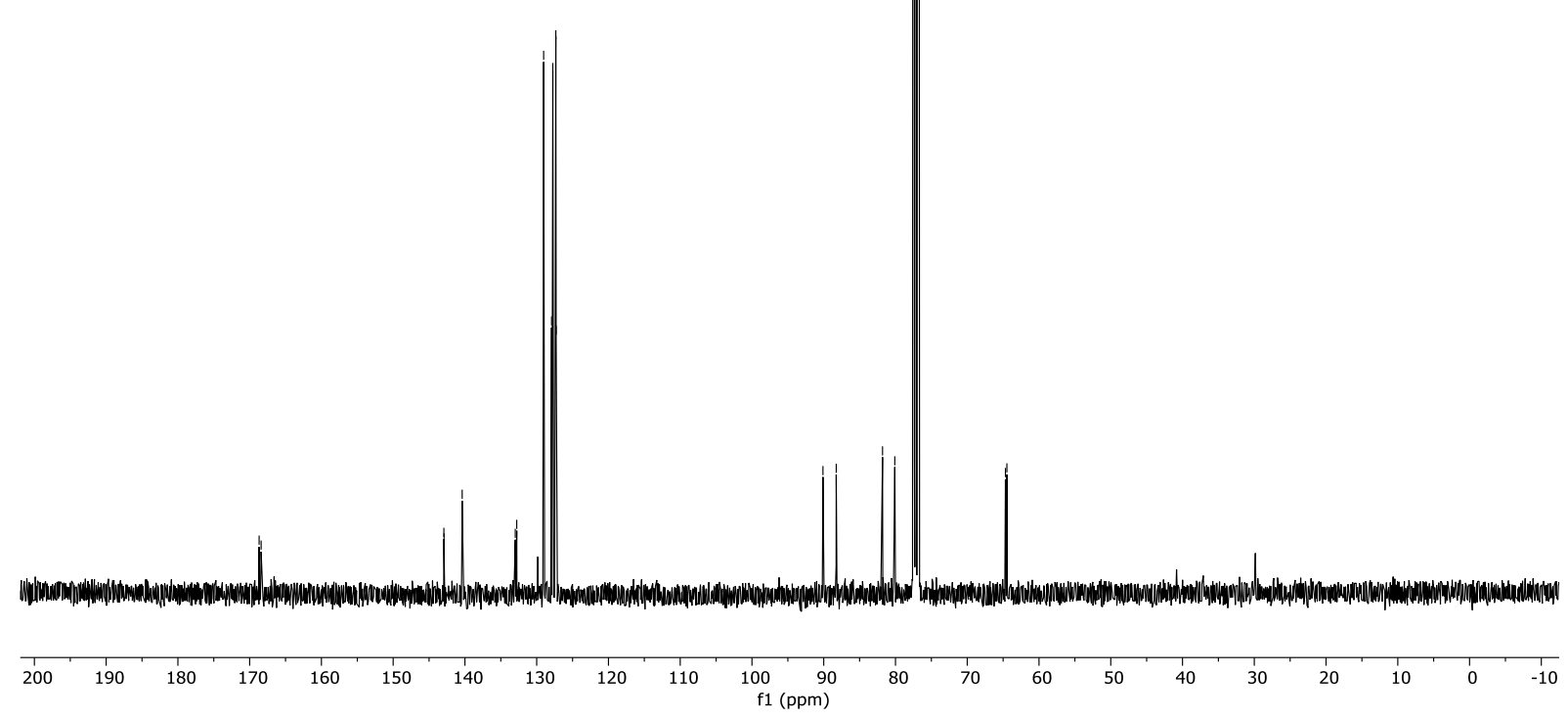


Divergent Synthesis of $\alpha$-Fluorinated Carbonyl and Carboxyl Derivatives by Double Electrophilic Activation of Amides

$$
\begin{aligned}
& \text { Internal } \\
& \text { standard (fluorobenzene) }
\end{aligned}
$$

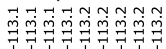

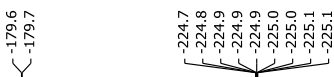

$\left(\mathrm{CDCl}_{3}, 376 \mathrm{MHz}\right)$

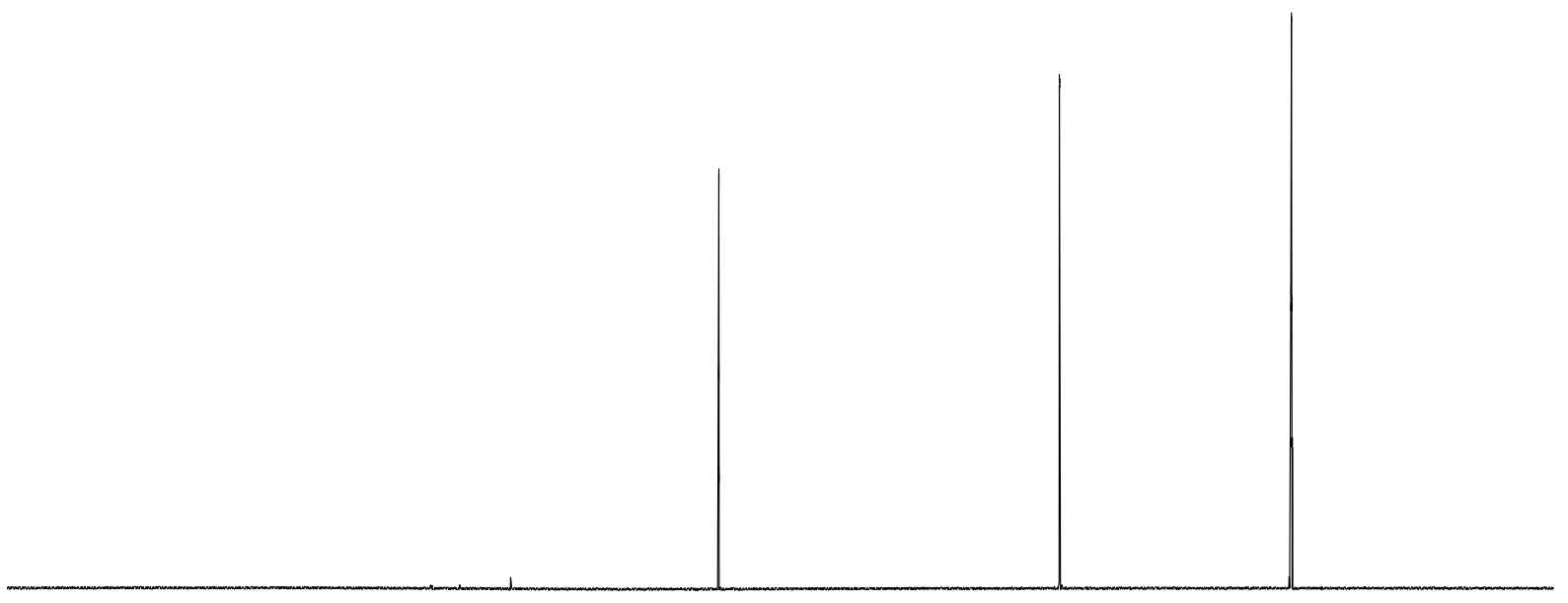

$\begin{array}{lllllllllllllllllllllllllllllllllllllllll}20 & 10 & 0 & -10 & -20 & -30 & -40 & -50 & -60 & -70 & -80 & -90 & -100 & -110 & -120 & -130 & -140 & -150 & -160 & -170 & -180 & -190 & -200 & -210 & -220 & -230 & -240 & -250 & -260 & -270\end{array}$ f1 (ppm) 


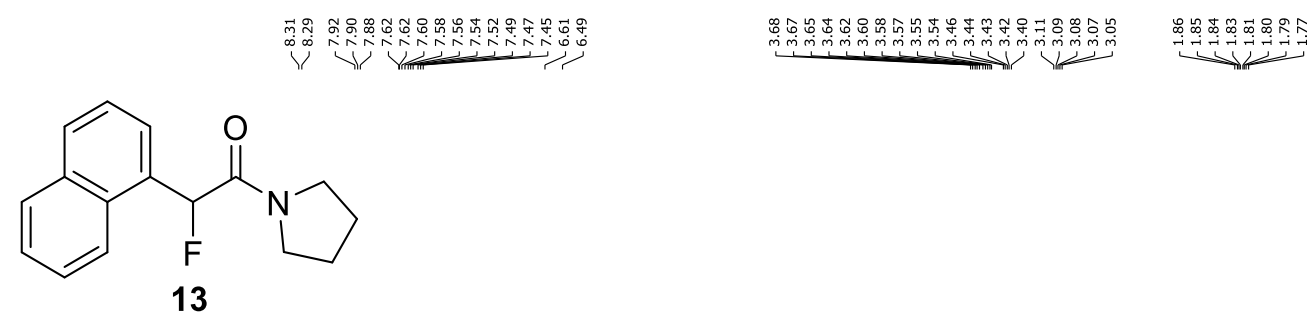

$\left(\mathrm{CDCl}_{3}, 400 \mathrm{MHz}\right)$
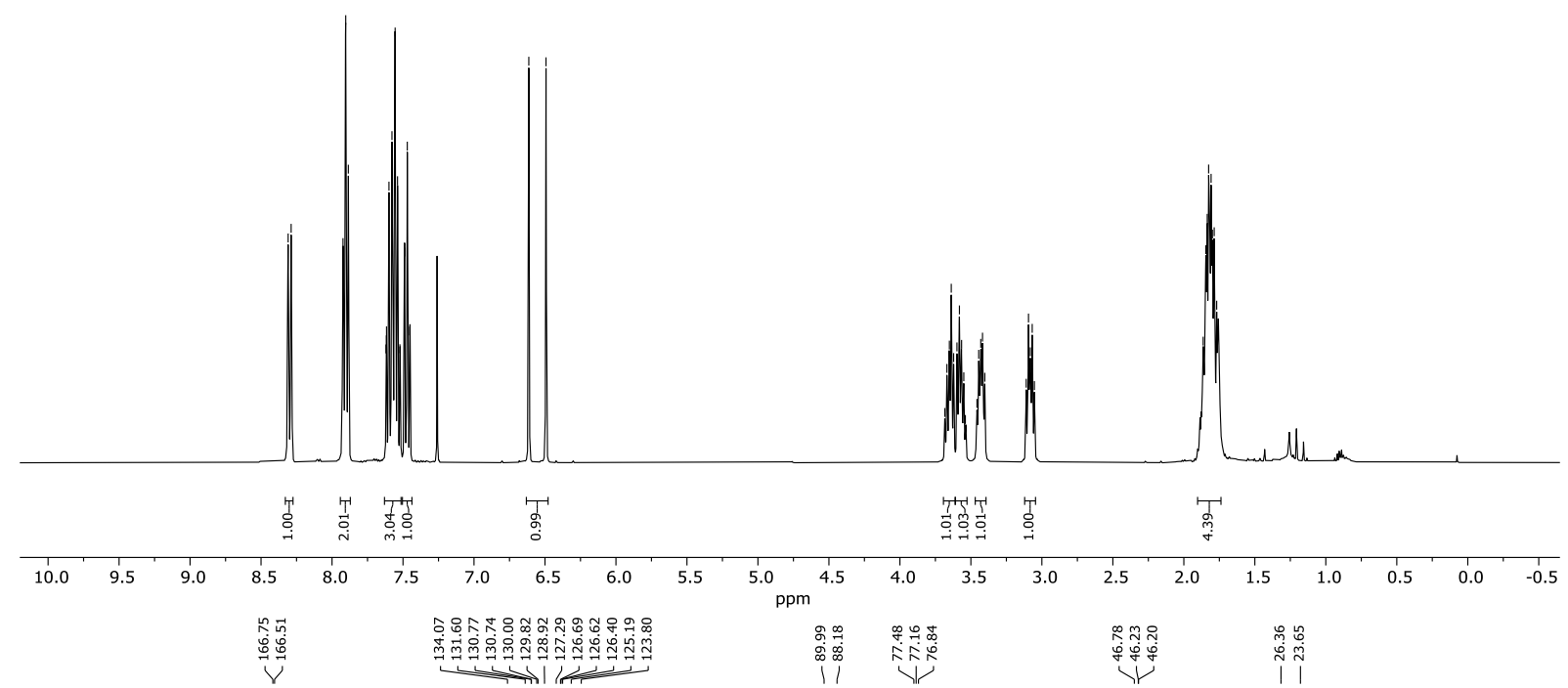

$\left(\mathrm{CDCl}_{3}, 100 \mathrm{MHz}\right)$

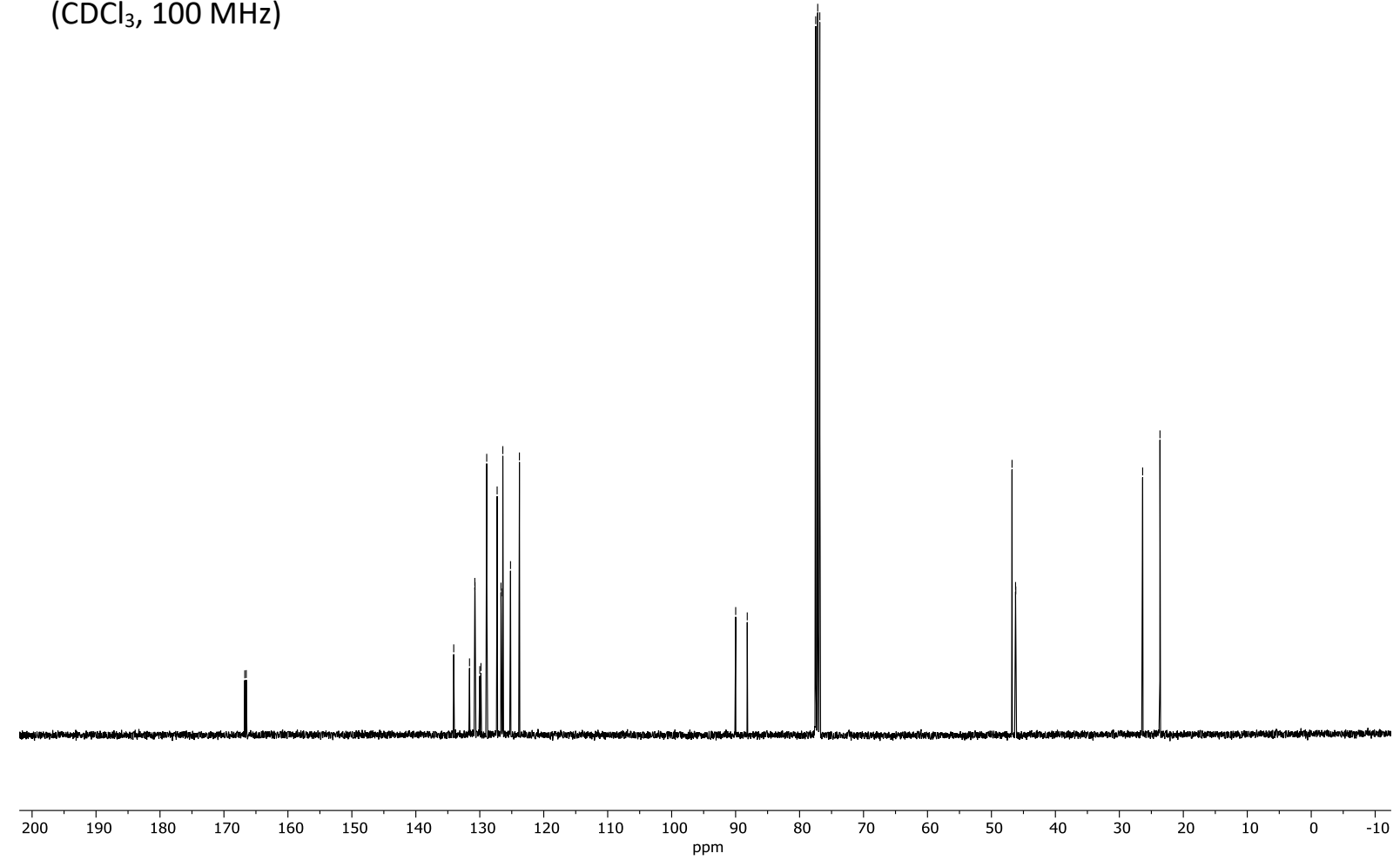


Divergent Synthesis of $\alpha$-Fluorinated Carbonyl and Carboxyl Derivatives by Double Electrophilic Activation of Amides

Internal

standard (fluorobenzene)

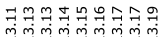

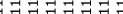

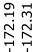

$\left(\mathrm{CDCl}_{3}, 376 \mathrm{MHz}\right)$

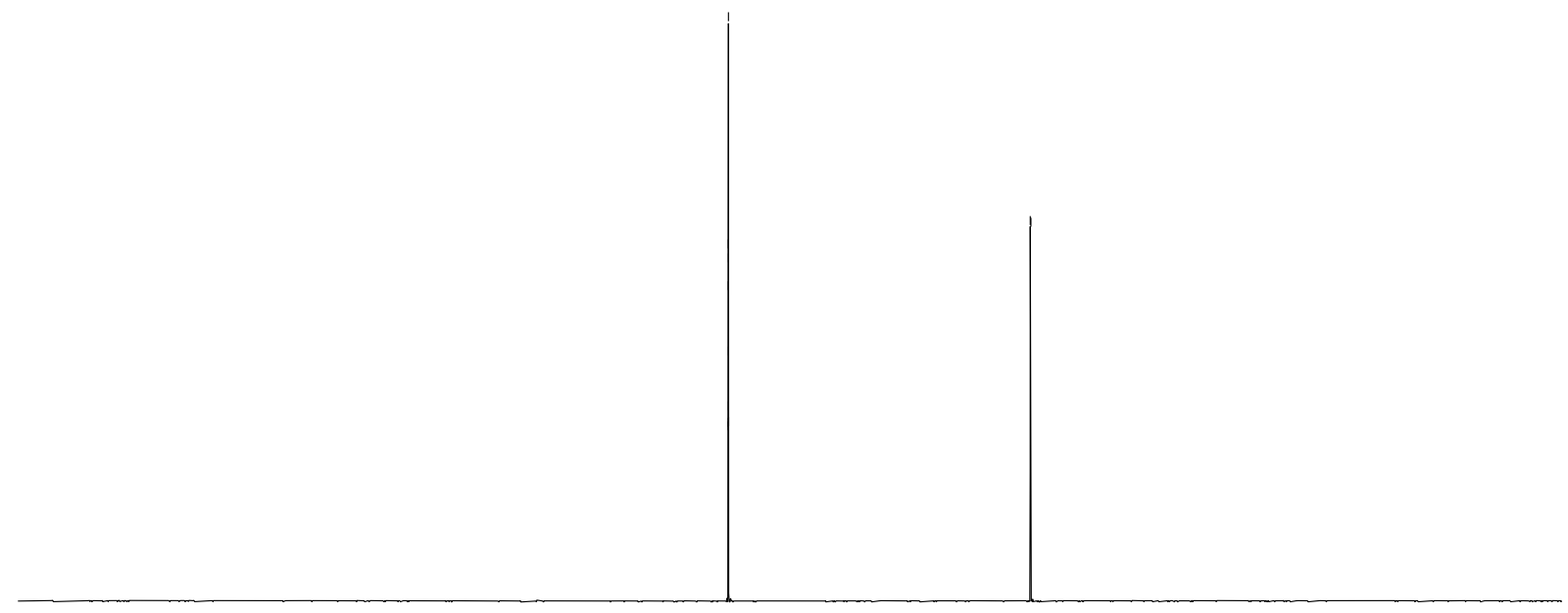

\begin{tabular}{llllllllllllllllllllllllllllllllllllllll}
\hline 20 & 10 & 0 & -10 & -20 & -30 & -40 & -50 & -60 & -70 & -80 & -90 & -100 & -110 & -120 & -130 & -140 & -150 & -160 & -170 & -180 & -190 & -200 & -210 & -220 & -230 & -240 & -250 & -260 & -270
\end{tabular} 

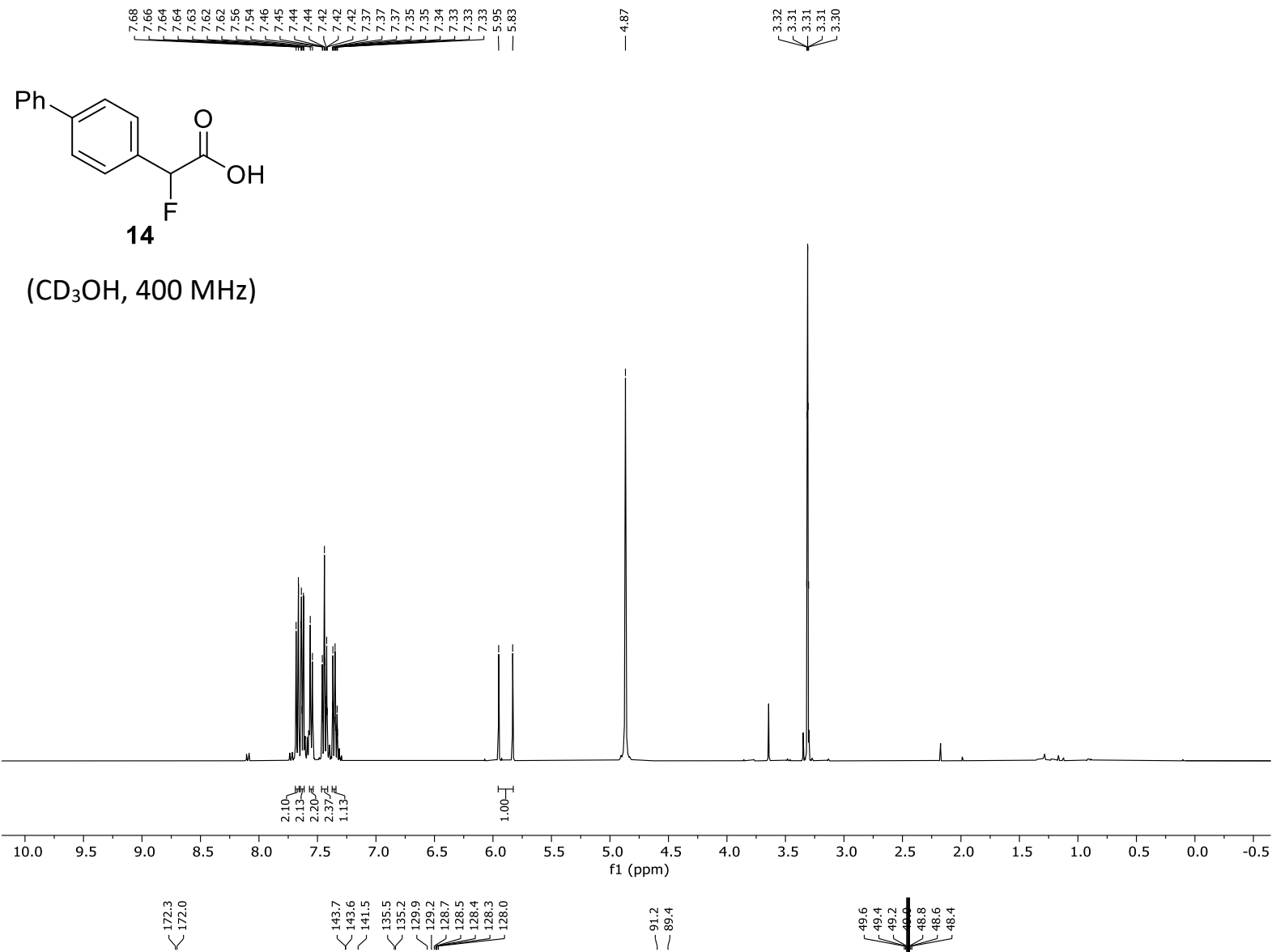

产品

$\left(\mathrm{CD}_{3} \mathrm{OH}, 100 \mathrm{MHz}\right)$

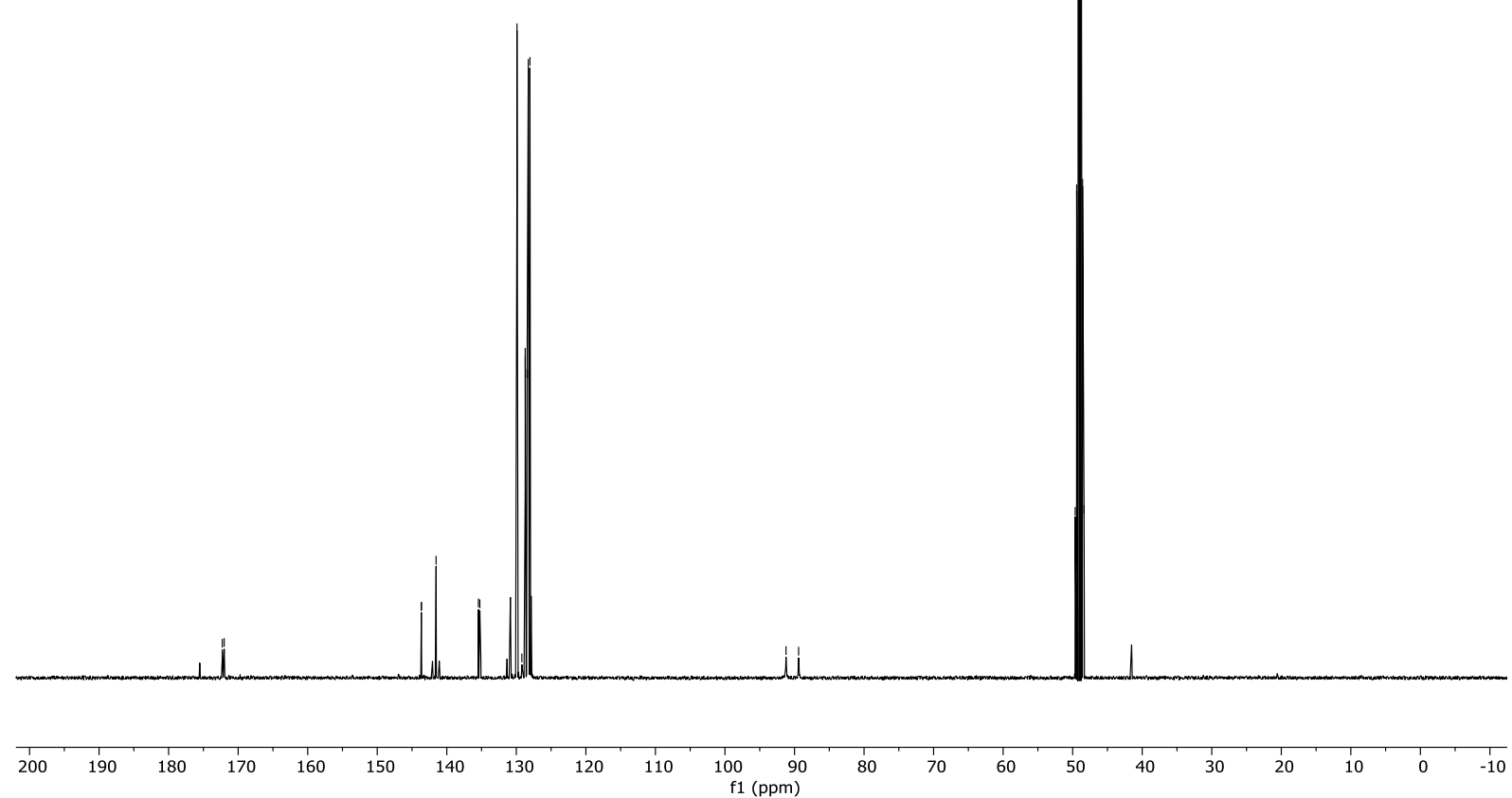


Divergent Synthesis of $\alpha$-Fluorinated Carbonyl and Carboxyl Derivatives by Double Electrophilic Activation of Amides

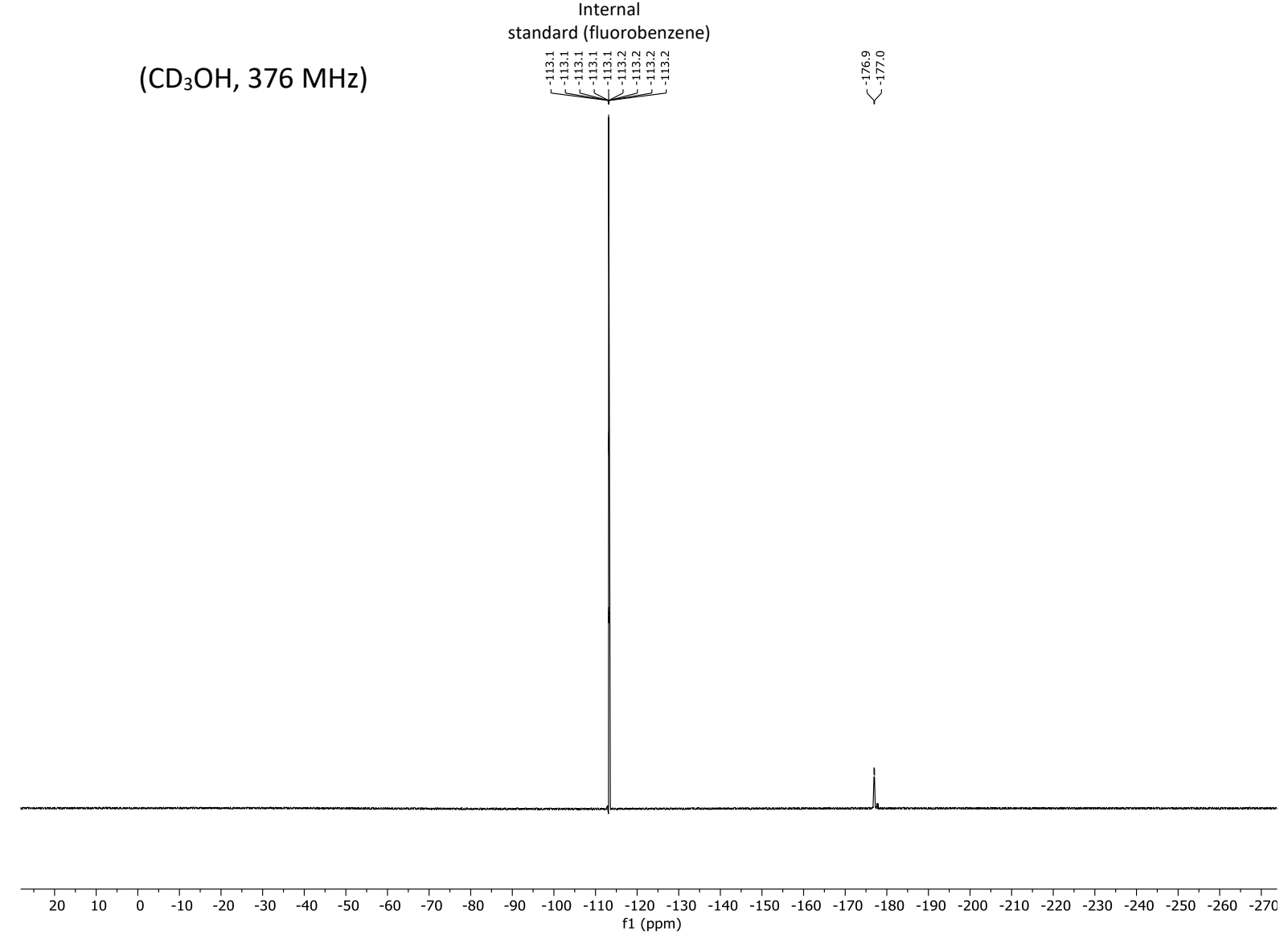




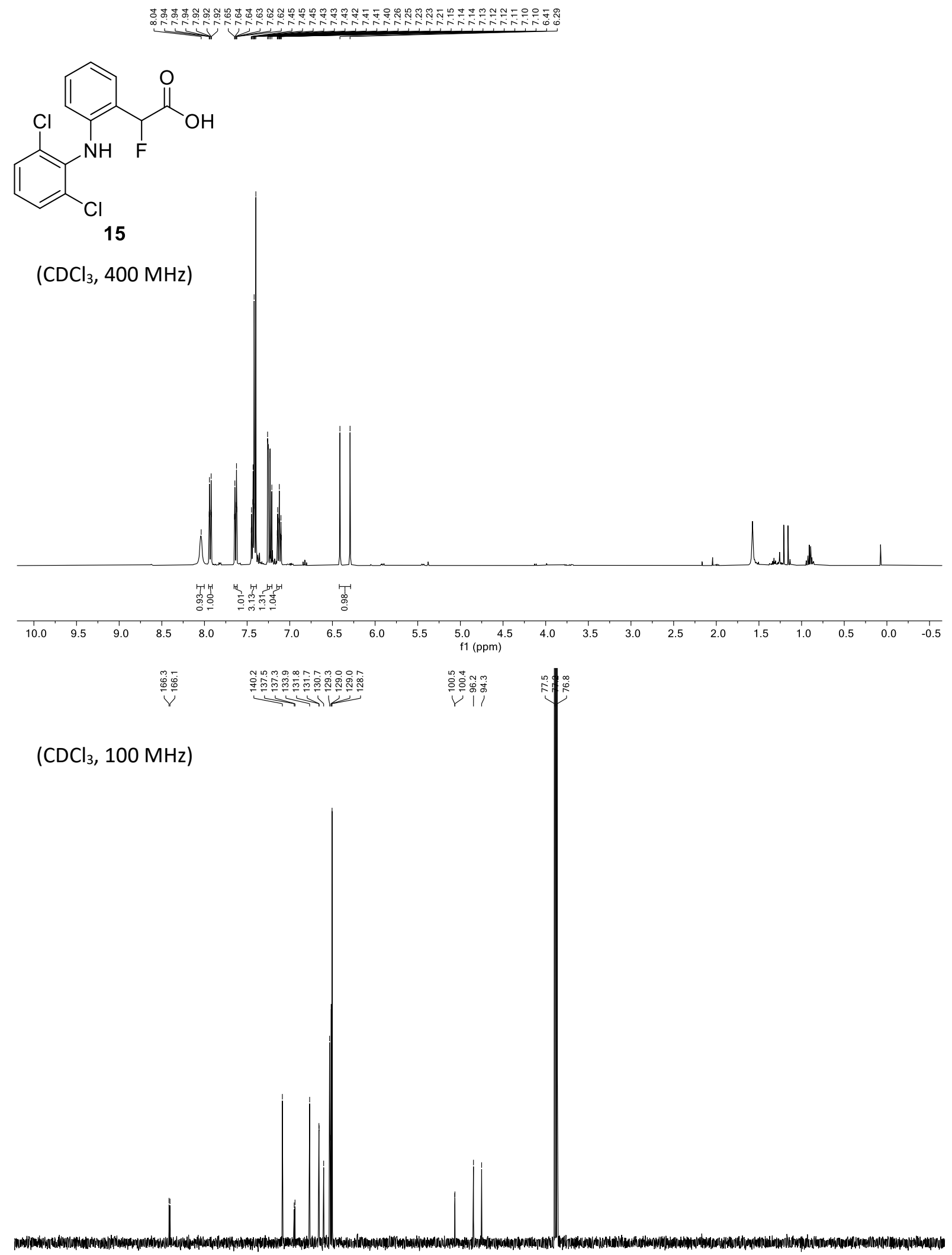

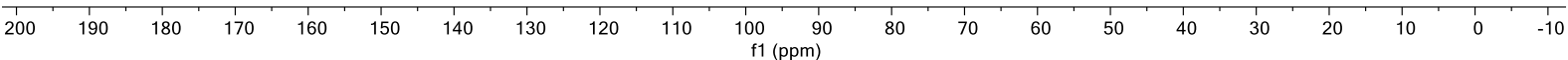


$\left(\mathrm{CDCl}_{3}, 376 \mathrm{MHz}\right)$

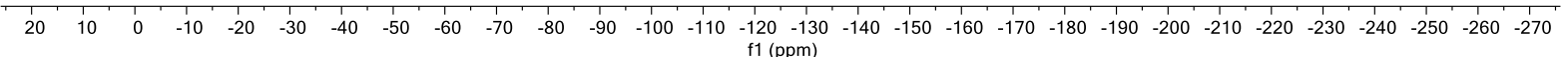

